Marcelo Roberto Baptista Pereira Luis Jimenez

\title{
VARIÁVEIS ALEATÓRIAS CÍCLICAS E SUA APLICAÇÃO NO ESTUDO DO RUÍDO INTERFEROMÉTRICO
}

\author{
Tese de Doutorado \\ Departamento de Engenharia Elétrica
}




\title{
VARIÁVEIS ALEATÓRIAS CÍCLICAS E SUA APLICAÇÃO NO ESTUDO DO RUÍDO INTERFEROMÉTRICO
}

\author{
Por \\ Marcelo Roberto Baptista Pereira Luis Jimenez \\ TESE APRESENTADA AO DEPARTAMENTO DE \\ ENGENHARIA ELÉTRICA DA PUC-RJ COMO PARTE \\ DOS REQUISITOS REQUISITOS NECESSÁRIOS PARA A \\ OBTENÇÃO DO GRAU DE DOUTOR EM CIÊNCIAS
}

Orientadores: Prof. Marco Antonio Grivet Mattoso Maia Prof. Jean Pierre von der Weid

PONTIFÍCIA UNIVERSIDADE CATÓLICA DO RIO DE JANEIRO RIO DE JANEIRO, 17 DE SETEMBRO DE 2001 
Para Wanda, Pepe

e Claudia. 


\section{Sumário}

Lista de Tabelas $\quad$ v

Lista de Figuras

Resumo viii

Abstract $\quad$ ix

Agradecimentos $\quad x$

Introdução 1

1 Variáveis Aleatórias Cíclicas $\quad 8$

1.1 Introdução . . . . . . . . . . . . . . . . . . . . . . . . . . 8

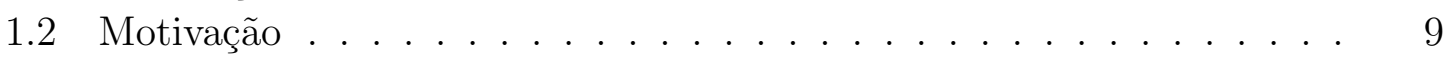

1.3 Medidas e Variáveis Aleatórias . . . . . . . . . . . . . . . . . . . . . . 10

1.4 O Grupo $\left(\mathbb{G}_{\Delta}, \oplus\right) \ldots \ldots \ldots \ldots \ldots$

1.5 Topologia em $\mathbb{G}_{\Delta} \ldots \ldots \ldots \ldots \ldots \ldots$

1.6 O Espaço Linear Topológico $\left(\mathbb{G}_{\Delta}, \oplus, \odot, g\right) \ldots \ldots$. . . . . . . . . . . . . . . . . . . . . . . .

$1.7 \sigma$-álgebra de sub-conjuntos de $\mathbb{G}_{\Delta} \ldots \ldots \ldots \ldots$

1.8 Variáveis Aleatórias em $\left(\mathbb{G}_{\Delta}, \oplus, \odot, g\right) \ldots \ldots$. . . . . . . . . . . . . . . . . . . . . . . . . . . .

1.9 Resultados Fundamentais . . . . . . . . . . . . . . . . 84

1.10 O Mapa $\mathbf{T}: \mathbf{M}^{+}\left(\mathbb{R}, \Sigma_{\mathbb{R}}\right) \longrightarrow \mathbf{M}^{+}\left(\mathbb{G}_{\Delta}, \Sigma_{\mathbb{G}_{\Delta}}\right) \ldots \ldots . \ldots . \ldots 92$

1.11 Séries de Fourier Cíclicas . . . . . . . . . . . . . . . . . . . . 93

1.12 Gaussianas Cíclicas . . . . . . . . . . . . . . . . . 96

2 Modelos Gaussianos de Ruído Interferométrico 100

2.1 Introdução . . . . . . . . . . . . . . . . . . . . . . . . . . 100

2.2 Ruído Interferométrico . . . . . . . . . . . . . . . . . . . . 100 
2.3 Expressão usual da literatura . . . . . . . . . . . . . . . . . . . . . . 101

2.4 Uma aproximação gaussiana mais realista . . . . . . . . . . . . . . . 107

2.5 Comparação entre os níveis de chão . . . . . . . . . . . . . . . . . . . 111

2.6 Usando outros níveis de decisão . . . . . . . . . . . . . . . . . . . . . 114

2.7 Apêndice I - Variância do produto . . . . . . . . . . . . . . . . . . . . 119

2.8 Apêndice II - Variância do ruído térmico ． . . . . . . . . . . . . . . . 120

2.9 Apêndice III - Ponto de decisão ótimo . . . . . . . . . . . . . . . . . . 120

2.10 Apêndice IV - Comparação entre os pontos de decisão . . . . . . . . . . 124

3 Modelo Não-Gaussiano de Ruído Interferométrico 126

3.1 Introdução . . . . . . . . . . . . . . . . . . . . . . . . 126

3.2 Cálculo da Densidade de Probabilidade do

Ruído Interferométrico . . . . . . . . . . . . . . . . . . . 127

3.3 Expressões das Transformadas de Fourier para o caso Gaussiano . . . 137

3.4 Programa para o Cálculo das Penalidades de Potência . . . . . . . . . 139

3.5 Apêndice I - Expressões Utilizadas . . . . . . . . . . . . . . . . . 146

4 Resultados Experimentais $\quad 148$

4.1 Introdução . . . . . . . . . . . . . . . . . . . . . . . . . . . 148

4.2 Descrição da montagem . . . . . . . . . . . . . . . . . . . . 148

4.3 Levantamento do parâmetro $\alpha$. . . . . . . . . . . . . . . . . 150

4.4 Número médio de erros, precisão da medida e tempo necessário . . . 151

4.5 Estratégia da medida . . . . . . . . . . . . . . . . . . . 154

4.6 O problema da responsividade do sensor . . . . . . . . . . . . . . . 156

4.7 Resultados obtidos para um crosstalk . . . . . . . . . . . . . . . . . 160

4.8 Resultados obtidos para dois crosstalks . . . . . . . . . . . . . . . . . 164

4.9 Resultados obtidos para 16 crosstalks . . . . . . . . . . . . . . . . . . . . . . . . . . . . . . . . . . .

4.10 Conclusões . . . . . . . . . . . . . . . . . . . . . . . . 168

$\begin{array}{llr}5 & \text { Conclusão } & 170\end{array}$

$\begin{array}{lc}\text { Referências Bibliográficas } & 173\end{array}$ 


\section{Lista de Tabelas}

4.1 Variação da Precisão em função do valor esperado do número de bits errados . . . . . . . . . . . . . . . . . . . . . . 153

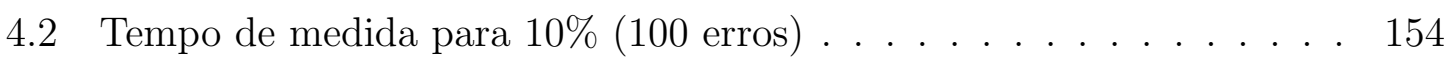




\section{Lista de Figuras}

1 Ruído Interferométrico . . . . . . . . . . . . . . . . . . . 1

2 Convolução de variáveis aleatórias angulares . . . . . . . . . . . . 5

$1.1 \quad$ Caso $m=0, \frac{a}{\Delta} \notin \mathbb{Z}, k \leqslant 1 \ldots \ldots \ldots$. . . . . . . . . . . 64

$1.2 \quad$ Caso $m=1, x_{1}=a, \frac{a}{\Delta} \in \mathbb{Z}, 0<k \leqslant 1 \ldots \ldots \ldots 65$

1.3 Diagrama dos conjuntos . . . . . . . . . . . . . . . 78

2.1 Comparação entre as duas parcelas e a soma da expressão (2.3.23) 107

2.2 Comparação entre os valores de probabilidade de erro sem crosstalk, o modelo Gaussiano simplificado, o modelo Gaussiano completo e o modelo proposto . . . . . . . . . . . . . . . . . 112

2.3 A razão entre $\mathrm{N}$ equivalente e $\mathrm{N}$ é aproximadamente 2 na maior parte da região . . . . . . . . . . . . . . . . . . . . . . . 114

2.4 Gráfico da probabilidade de erro em função do nível de decisão. O ponto de máximo é de difícil visualização, e encontra-se à esquerda do valor mínimo . . . . . . . . . . . . . . . . . . . . . . . . 122

2.5 Gráfico da probabilidade de erro em função do nível de decisão, ressaltando o ponto de máximo . . . . . . . . . . . . . . . . . . . . . 123

2.6 Gráfico da probabilidade de erro em função do nível de decisão, mostrando o ponto de mínimo calculado usando o ponto de decisão ótimo 123

2.7 Comportamento da função Q $(\mathrm{x}) \ldots \ldots$. . . . . . . . . . 125

3.1 Gráfico da densidade de probabilidade do cosseno de uma v.a. uniforme128 
3.2 Modelo Não-Gaussiano x Simplificado - Polarizações Alinhadas . . . . 140

3.3 Modelo Gaussiano x Simplificado - Polarizações Alinhadas . . . . . . 141

3.4 Modelo Gaussiano x Não-Gaussiano - Polarizações Alinhadas . . . . . 142

3.5 Modelo Não-Gaussiano x Simplificado - Polarizações Não-Alinhadas · 143

3.6 Modelo Gaussiano x Simplificado - Polarizações Não-Alinhadas . . . . 144

3.7 Modelo Gaussiano x Não-Gaussiano - Polarizações Não-Alinhadas . 145

4.1 Esquema do experimento . . . . . . . . . . . . . . . . . . . . . . . . . 149

4.2 Gráfico da probabilidade de erro em função da relação sinal-ruído elétrica, sem compensação de responsividade . . . . . . . . . . . . . . 157

4.3 Gráfico da probabilidade de erro em função da relação sinal-ruído elétrica, com compensação de responsividade . . . . . . . . . . . . . . 159

4.4 Histograma do sinal . . . . . . . . . . . . . . . . . . . . . . . . . . . 161

4.5 Comparação dos valores teóricos e medidos da probabilidade de erro em função da relação sinal-ruído elétrica, 1 crosstalk, $\alpha=-13 \mathrm{~dB}$. . 162

4.6 Comparação dos valores teóricos e medidos da probabilidade de erro em função da relação sinal-ruído elétrica, 1 crosstalk, $\alpha=-23 \mathrm{~dB}$. . 163

4.7 Comparação dos valores teóricos e medidos da probabilidade de erro em função da relação sinal-ruído elétrica, 1 crosstalk, $\alpha=-33 \mathrm{~dB}$. . 164

4.8 Comparação dos valores teóricos e medidos da probabilidade de erro em função da relação sinal-ruído elétrica, 2 crosstalks, $\alpha=-18.32 \mathrm{~dB}$

4.9 Comparação dos valores teóricos e medidos da probabilidade de erro em função da relação sinal-ruído elétrica, 2 crosstalks, $\alpha=-24.32 \mathrm{~dB}$

4.10 Comparação dos valores teóricos e medidos da probabilidade de erro em função da relação sinal-ruído elétrica, 2 crosstalks, $\alpha=-30.32 \mathrm{~dB}$

4.11 Comparação dos valores teóricos e medidos da probabilidade de erro em função da relação sinal-ruído elétrica, 16 crosstalks, $\alpha=-31.45 \mathrm{~dB} 168$ 


\section{Resumo}

O ruído interferométrico é um fator limitante cada vez mais importante nos sistemas óticos, principalmente nas ligações de longa distância em redes óticas transparentes. O presente trabalho analisa modelos para este tipo de ruído, dando um tratamento matemático novo para o modelo não-gaussiano. A teoria matemática é desenvolvida em detalhes e com rigor. O modelo gaussiano foi usado a fim de fazer previsões quanto aos valores de chão da taxa de erro de bits. Os dois modelos foram simulados em computador e comparados com os testes realizados em laboratório e os resultados são apresentados. 


\section{Abstract}

The interferometric noise is becoming a serious limiting factor in optical systems, notably on long distance connections in transparent optical networks. The present work analyzes models for this kind of noise, giving a new mathematical treatment to the non-gaussian model. The mathematical theory is developed in detail and rigorously. The gaussian model was used in order to make predictions relative to bit error rate floors. Both models were simulated in computer and compared with the tests made in laboratory and the results are presented. 


\section{Agradecimentos}

Gostaria de agradecer aos professores Rogério Passy e Jean Pierre von der Weid por suas diversas sugestões e pela ajuda constante durante esta pesquisa. Agradeço também aos professores Marco Antonio Grivet Mattoso Maia e Carlos Kubrusly pela ajuda e paciência de aturar minhas idéias malucas.

Sou grato também a meu pai e minha irmã pela paciência que tiveram durante todos esses anos em que estive envolvido neste trabalho.

Gostaria de agradecer também a: Alexandre Dalforno, Atila Xavier, José Augusto da Silva ( Guto ), Miguel Freitas e Rodolfo Saboia pela amizade e pelo apoio.

Não poderia esquecer de agradecer também a todo o pessoal do laboratório de ótica que sempre me ajudou nos momentos difíceis da bancada.

Agradeço também a todo o corpo técnico do CETUC, que sempre me apoiou, tanto no trabalho quanto na tese.

Finalmente, quero agradecer a todos os meus amigos do Clube Excursionista Carioca, pois o sentido verdadeiro da palavra amizade está ali.

Rio de Janeiro, RJ

Marcelo Roberto Jimenez

17 de Setembro de 2001 


\section{Introdução}

O assunto desta dissertação é ruído interferométrico, ou ruído de multipercurso. Esse é o nome dado ao ruído produzido em sistemas óticos devido à alguma descontinuidade do índice de refração do meio onde a luz se propaga ( Figura 1 ). Tais descontinuidades ocorrem normalmente devido à presença de dispositivos óticos ou conectores. A variação abrupta do índice de refração de um meio provoca um fenômeno análogo ao mau casamento de impedâncias nos sistemas elétricos, fazendo com que uma parte do sinal siga na direção correta, enquanto outra parte do sinal é refletida na direção contrária à desejada [14]. Uma parte do sinal refletido pode, por sua vez, ser refletido de volta na direção desejada por uma descontinuidade anterior, e agora como está no sentido "correto", passa a interferir com a informação transmitida, sendo portanto indesejável. A conseqüência imediata deste fenômeno é um aumento da taxa de bits errados do sistema, ou seja, uma degradação do desempenho do sistema.

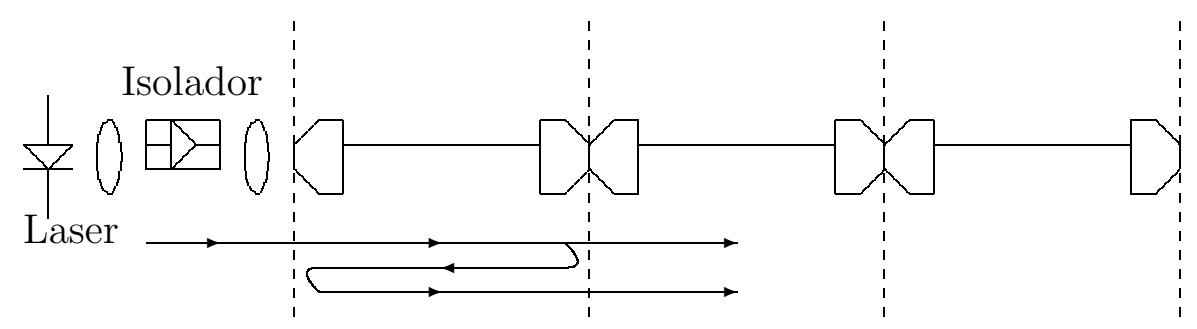

Figura 1: Ruído Interferométrico 
Os problemas associados ao ruído interferométrico são mais importantes nas redes óticas transparentes. Estas redes são enlaces longos em que a atenuação ótica é periodicamente compensada por amplificadores óticos, sem que haja a necessidade de usar regeneradores. Os regeneradores são estágios intermediários onde é realizada uma conversão opto-elétrica, um processo de decisão binária ( regeneração ) e uma conversão eletro-ótica. O advento dos amplificadores óticos foi o fator que possibilitou a eliminação dos regeneradores das redes óticas. Tais amplificadores podem ser configurados para gerar níveis de ruído extremamente baixos, desde que os ganhos individuais de cada estágio amplificador mantenham-se pequenos o suficiente [16]. Porém, a não utilização dos estágios regeneradores faz com que o ruído interferométrico, antes eliminado nestes estágios, passe a se acumular na rede como um todo, dando origem a um fenômeno chamado na literatura de BER Floor. Esse nome refere-se ao fato de que por mais que se aumente a potência do sinal transmitido, a taxa de bits errados do sistema não melhora significativamente, ou seja, a taxa de bits errados atinge um "valor de chão". Desta forma, o ruído interferométrico passa a ser um fator limitante no projeto deste tipo de rede ótica.

O uso de sistemas WDM ( Wavelength Division Multiplexing) também implica em penalidades devido a sinais de um canal que passam para os canais adjacentes. Tal fenômeno é chamado de crosstalk. O crosstalk ocorre geralmente nos chamados multiplexadores add-drop, que fazem o papel de roteadores das redes óticas transparentes. Nesses multiplexadores os sinais óticos provenientes de diversas fontes são "re-arranjados" nos diversos comprimentos de onda de modo a suprimir os sinais que devem sair da rede num determinado nó e a conter os sinais que devem entrar na rede neste mesmo nó, além de manter os sinais que devem continuar o seu trajeto 
em direção aos próximos nós [12]. Conforme se pode ver em [31], o tratamento matemático do fenômeno de crosstalk é completamente análogo em forma ao tratamento dado ao ruído interferométrico devido à reflexões multiplas em conectores mencionado anteriormente, de modo que toda a teoria aqui desenvolvida serve como modelo para ambos os fenômenos.

Um dos primeiros trabalhos nessa área de pesquisa é o trabalho de James L. Gimlett e Nim K. Cheung [14]. Neste artigo, os autores fazem uma análise da distribuição de probabilidade do ruído interferométrico em sistemas óticos. A distribuição de probabilidade para o caso de um sinal principal e um sinal interferente é rapidamente mostrada, mas devido à dificuldade de se obterem expressões analíticas utilizando este modelo, vários resultados teóricos são obtidos utilizando-se uma aproximação gaussiana. O principal problema deste tipo de aproximação é que para poucos sinais interferentes ela é inadequada. Isto pode ser visto claramente pela forma da curva da distribuição de probabilidade teórica do ruído interferométrico para um sinal interferente. Neste caso, a curva teórica é limitada e tem dois picos, enquanto a aproximação gaussiana é ilimitada e tem apenas um pico. Porém, espera-se que a medida que o número de sinais interferente cresça, a distribuição de probabilidade do ruído resultante comece a se parecer mais com uma distribuição gaussiana, devido ao teorema do limite central.

A suposição de que no caso de vários sinais interferentes o comportamento do ruído interferométrico pode ser aproximado por uma distribuição gaussiana foi utilizado posteriormente por Peter J. Legg e outros [27] e também por W. D. Cornwell e I. Andonovic [6]. Porém, no caso de poucos sinais interferentes, a ausência de uma expressão analítica para a distribuição de probabilidade do ruído interferométrico 
continuava sendo um problema. A distribuição de probabilidade para o ruído interferométrico obtida no trabalho de Cornwell pode ser escrita como a convolução de distribuições de probabilidade idênticas. Porém, o método utilizado neste trabalho para fazer o cálculo numericamente foi o de executar diretamente a convolução. Para se obter resultados numéricos de boa precisão, o número de pontos necessários nessa convolução deve ser bastante grande, o que faz com que os cálculos por computador demorem muito tempo.

L. Moura e outros [31], aplicaram o problema do ruído interferométrico a redes WDM, e nele aparece uma técnica interessante para o cálculo da distribuição de probabilidade do ruído interferométrico que utiliza funções geradoras de momento a fim de evitar o cálculo da convolução. Também é apresentado um método para calculo de um limitante para a probabilidade de erro do sistema, utilizando uma técnica chamada de MCB ( Modified Chernoff Bound), isto é, usa um limitante de Chernoff modificado. A teoria do MCB pode ser encontrada no no trabalho de John J. O’Reilly e José R. F. da Rocha [33]. Apesar de não ser uma solução analítica fechada para o problema, esta abordagem fornece um limite interessante para os valores de probabilidade de erro do sistema, além de não sofrer do problema de demora do cálculo da convolução.

Na linha da aproximação utilizando o modelo mais exato, isto é, o não-gaussiano, existe o trabalho de Idelfonso T. Monroy e Eduward Tangdionga [29]. A técnica empregada por eles utiliza também funções geradoras de momento além da técnica de aproximações por ponto de sela proposta em [20].

Vale a pena ressaltar que de modo geral, o uso de variáveis aleatórias que representam ângulos tem uma falha conceitual que pode ser exemplificada na seguinte 
situação: imagine duas variáveis aleatórias uniformes e independentes no intervalo $[-\pi, \pi]$, e calcule a distribuição de probabilidade de uma nova variável aleatória que é a soma das duas anteriores. Usando a teoria das variáveis aleatórias reais, é fácil mostrar que a variável aleatória resultante possui densidade de probabilidade não-nula no intervalo $[-2 \pi, 2 \pi]$ e não mais apenas no intervalo $[-\pi, \pi]$ ( Figura 2 ). Ela "se espalha" por ângulos equivalentes. O mesmo acontece quando se usa uma variável aleatória gaussiana para modelar uma variável angular. Tal distribuição assume valores na reta real inteira, sendo portanto inadequada a este tipo de variável. Existe uma identificação implícita entre os ângulos da forma $\alpha+2 k \pi$, onde $k$ é um número inteiro. É nesse ponto que entram as variáveis aleatórias cíclicas. Esta teoria foi criada de modo a suprir a deficiência das variáveis aleatórias reais na modelagem de ângulos.
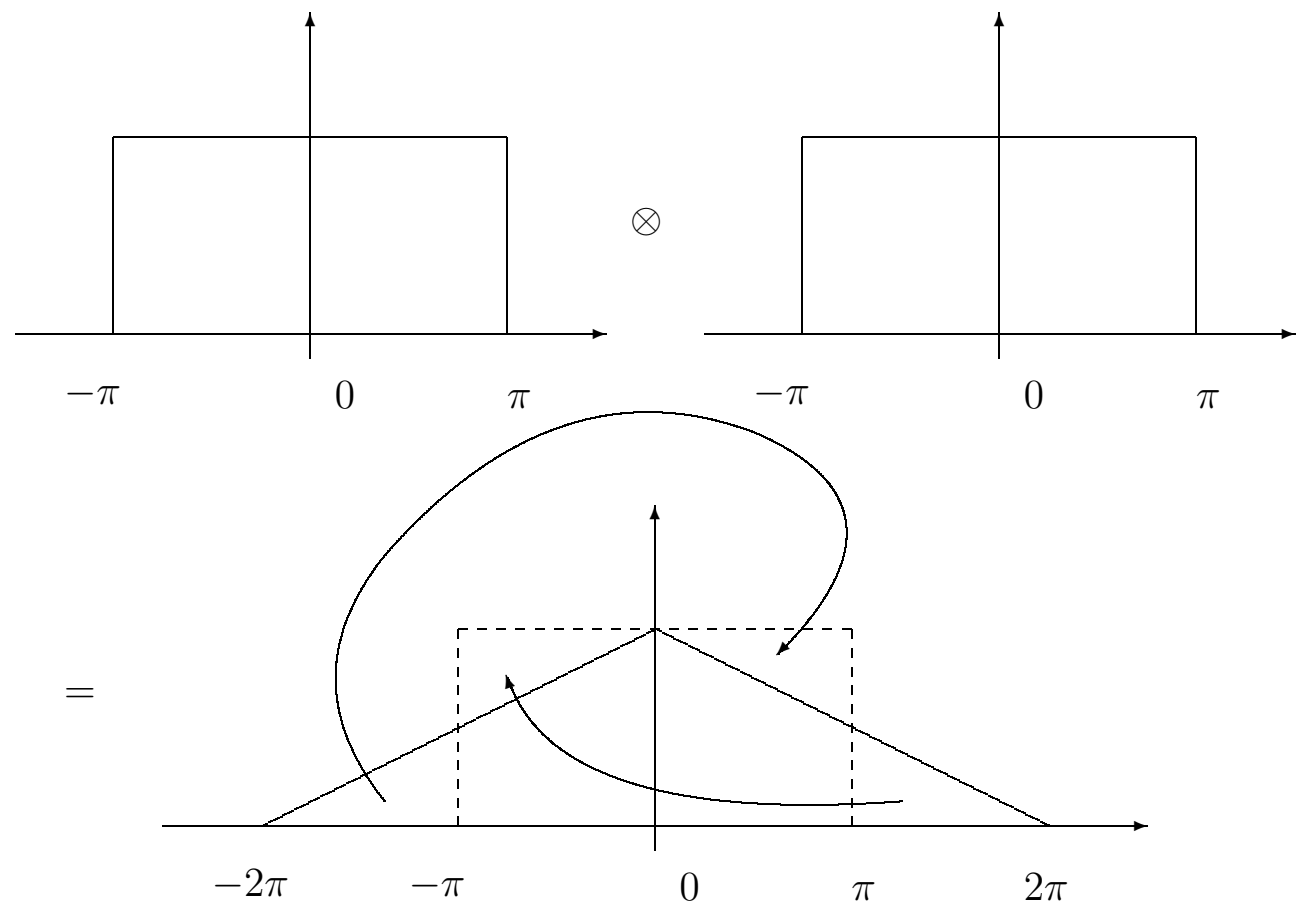

Figura 2: Convolução de variáveis aleatórias angulares 
O objetivo deste trabalho é fazer uma análise teórica e prática de modelos estatísticos para o ruído interferométrico a fim de que seja possível fazer previsões realistas a respeito das taxas de bits errados e BER Floors dos sistemas óticos. Neste trabalho foram obtidos alguns resultados teóricos que permitiram a obtenção de expressões analíticas para determinados casos de ruído interferométrico para os quais o autor não tem conhecimento de resultado anterior semelhante. A teoria matemática necessária para a obtenção destes resultados ( variáveis aleatórias cíclicas ) é desenvolvida em detalhes no capítulo 1 , e os resultados obtidos serão utilizados nos capítulos subsequentes, sendo esta uma contribuição original deste trabalho. No capítulo 2 é analisado o modelo gaussiano de ruído interferométrico. Conforme foi dito anteriormente, este é um modelo muito utilizado na literatura devido à facilidade de obtenção de resultados analíticos $[14,16,15,17,27,6,5]$. Vários resultados obtidos na literatura são re-examinados e estendidos neste capítulo. Os modelos gaussianos são ainda utilizados no capítulo 2 para fazer a previsão de "BER Floors", ou seja, valores de chão de taxa de erro de bits, sendo esta também uma contribuição original deste trabalho. No capítulo 3 é estudado o modelo não-gaussiano, mais realista no que diz respeito à estatistica do sinal, que é claramente não-gaussiana [14, 26]. Expressões analíticas fechadas para a função densidade de probabilidade foram obtidas com o auxílio da teoria das variáveis aleatórias cíclicas. De posse destas expressões, um programa de simulação foi feito para se obter as penalidades de potência previstas tanto pelo modelo gaussiano quanto pelo modelo não-gaussiano. Esse programa de simulação é na verdade a origem deste trabalho. Ele foi feito e gradativamente aprimorado a fim de realizar eficientemente o cálculo teórico das penalidades de potência. O cálculo original era realizado através de uma convolução, o que tornava o método 
muito lento. Neste programa foram utilizadas técnicas de transformada de Fourier, sendo esta uma contribuição original. Técnicas semelhantes podem ser encontradas em [31], só que utilizando a função geradora de momentos no lugar da transformada de Fourier. No capítulo 4 foi feita a descrição do experimento montado a fim de avaliar a utilidade dos modelos aqui estudados e os dados obtidos experimentalmente foram analisados, comparando com os modelos desenvolvidos nos capítulos anteriores. Finalmente no capítulo 5 estão as conclusões. 


\section{Capítulo 1}

\section{Variáveis Aleatórias Cíclicas}

\subsection{Introdução}

Neste capítulo será formalizado o modelo matemático que será utilizado nos capítulos subsequentes para atribuir corretamente probabilidades a ângulos. A motivação para este tipo de abordagem advém do fato que o uso de variáveis aleatórias definidas no conjunto dos números reais para modelar variáveis tais como ângulos, pode esconder determinadas propriedades inerentes a este tipo de variável, propriedades estas que não possuem análogos nas variáveis aleatórias reais ordinárias. Os ângulos possuem uma característica periódica que não está presente nas variáveis aleatórias reais ordinárias. Tal característica faz surgir resultados inesperados, que permitirão a obtenção de expressões fechadas para densidades de probabilidade que, convencionalmente, não poderiam ser facilmente calculadas. No conjunto de variáveis aleatórias cíclicas a ser definido e sob determinadas condições é possível somar duas variáveis aleatórias e o resultado ser uma variável aleatória independente de uma ou de ambas as parcelas.

O conceito de variável aleatória será discutido sob a luz da teoria de medidas. 
Apesar do formalismo matemático aqui empregado ser maior que o adotado normalmente em livros de engenharia, os resultados obtidos são relativamente simples de serem usados. Tal formalismo é necessário a fim de conferir solidez à teoria aqui desenvolvida.

\subsection{Motivação}

Sejam $\alpha$ e $\beta$ duas variáveis aleatórias reais representando ângulos. Suponha então que numa determinada situação deseja-se saber qual a distribuição de probabilidade da variável $A=\cos (\alpha+\beta)$. Pode-se mostrar pela teoria das variáveis aleatórias que se $\alpha$ e $\beta$ forem variáveis aleatórias independentes, a função densidade de probabilidade da variável aleatória $\gamma=\alpha+\beta$ é a convolução das funções densidade de probabilidade das variáveis $\alpha$ e $\beta$. Pode-se mostrar também que se $\gamma$ for uniforme no intervalo $[-\pi, \pi]$, então a função densidade de probabilidade da variável aleatória $A$ pode ser escrita como:

$$
p_{A}(a)=\frac{1}{\pi \sqrt{1-a^{2}}},|a|<1
$$

Suponha então que tanto $\alpha$ como $\beta$ sejam variáveis aleatórias independentes e uniformes no intervalo $[-\pi, \pi]$. Então, a função densidade de probabilidade da variável aleatória $\gamma$ será um triângulo isósceles cuja base é o intervalo $[-2 \pi, 2 \pi]$, e a

altura vale $\frac{1}{2 \pi}$. Antes de tentar descobrir qual é a função densidade de probabilidade da variável aleatória $A$, note que no caso dos ângulos existe uma relação de equivalência implícita entre ângulos da forma $\gamma+2 \pi n, n \in \mathbb{Z}$, o que significa que no triângulo isósceles que representa a função densidade de probabilidade da variável aleatória $\gamma$, todos os ângulos estão representados duas vezes. A fim de remover esta 
ambigüidade, mantém-se o intervalo original de $[-\pi, \pi]$ e soma-se as probabilidades dos ângulos $\gamma+2 \pi n, \gamma \in[-\pi, \pi], n \in\{-1,0,1\}$. Nestas condições, o triângulo isósceles transforma-se num retângulo, de modo que a variável aleatória $\gamma$ é na verdade uniforme no intervalo $[-\pi, \pi]$. Note como este resultado é interessante: a soma de duas variáveis aleatórias uniformes resultando numa outra variável aleatória uniforme. Mais interessante ainda é que, como será mostrado adiante, a variável aleatória soma, é independente de uma das parcelas, apesar de não ser independente de ambas as parcelas simultaneamente. Obviamente não é qualquer variável aleatória uniforme que possui esta propriedade, sendo a estrutura cíclica dos ângulos o que faz com que isto se manifeste. Na verdade, o modelo de variável aleatória utilizado para ângulos não é muito coerente do ponto de vista formal, pois se for permitido que os ângulos variem no intervalo $(-\infty, \infty)$, não é possível existir uma variável aleatória uniforme no sentido que a função densidade de probabilidade tem o mesmo valor em todos os pontos, uma vez que esta função constante não seria normalizável. Por outro lado, atendo-se ao intervalo $[-\pi, \pi]$, por exemplo, toda vez que se deseje fazer uma conta com variáveis aleatórias angulares, no final o resultado deverá ser reduzido ao intervalo original, para que as probabilidades não se espalhem por ângulos equivalentes.

A modelagem das variáveis angulares proposta a seguir, leva em conta desde o princípio a característica cíclica dos ângulos, e isto permitirá ver com clareza as propriedades particulares deste tipo de variável aleatória.

\subsection{Medidas e Variáveis Aleatórias}

Definição 1.3.1. Topologia. Seja $X$ um conjunto, e seja $\mathcal{T}_{X}$ um subconjunto de $2^{X}$. Então $\mathcal{T}_{X}$ é dita uma topologia se satisfizer às seguintes propriedades: 
i) $\emptyset \in \mathcal{T}_{X}$ e $X \in \mathcal{T}_{X}$

ii) $A_{\alpha} \in \mathcal{T}_{X} \Rightarrow \bigcup_{\alpha} A_{n} \in \mathcal{T}_{X}$

iii) $\forall n \in \mathbb{N}, A_{n} \in \mathcal{T}_{X} \Rightarrow \bigcap_{n=1}^{\infty} A_{n} \in \mathcal{T}_{X}$.

Os elementos de $\mathcal{T}_{X}$ são chamados de conjuntos abertos.

Definição 1.3.2. $\sigma$-álgebra. Seja $X$ um conjunto, e seja $\Sigma_{X}$ um subconjunto de $2^{X}$. Então $\Sigma_{X}$ é dita uma $\sigma$-álgebra se satisfizer às seguintes propriedades:

i) $\emptyset \in \Sigma_{X}$

ii) $A \in \Sigma_{X} \Leftrightarrow A^{\prime}=X-A \in \Sigma_{X}$

iii) $\forall n \in \mathbb{N}, A_{n} \in \Sigma_{X} \Rightarrow \bigcup_{n=1}^{\infty} A_{n} \in \Sigma_{X}$

Sejam $X$ e $Y$ conjuntos quaisquer, e sejam $\Sigma_{X}$ e $\Sigma_{Y} \sigma$-álgebras geradas a partir dos conjuntos $X$ e $Y$ respectivamente. Quando se fala de probabilidade, elementos das $\sigma$ álgebras são chamados de eventos. Seja ainda $\mu$ uma medida sobre $\Sigma_{X}$ tal que $\mu(X)=$ 1, isto é, $\mu$ é uma medida de probabilidade. Sejam então os espaços mensuráveis $\left(X, \Sigma_{X}\right)$ e $\left(Y, \Sigma_{Y}\right)$. Uma função $f:\left(X, \Sigma_{X}\right) \longrightarrow\left(Y, \Sigma_{Y}\right)$ é dita mensurável, se a imagem inversa de qualquer elemento de $\Sigma_{Y}$ através de $f$ for um elemento de $\Sigma_{X}$, isto é, $\forall B \in \Sigma_{Y}, A=f^{-1}(B) \in \Sigma_{X}$. A função mensurável $f$ juntamente com a medida $\mu$ induzem no espaço mensurável $\left(Y, \Sigma_{Y}\right)$ uma nova medida $\nu$ definida por: $\forall B \in \Sigma_{Y}, \nu(B)=\mu\left(f^{-1}(B)\right)$. Tal medida está bem definida, uma vez que como a função $f$ é mensurável, a imagem inversa de um elemento de $\Sigma_{Y}$ pertence à $\Sigma_{X}$. Como $f$ é uma função, todo elemento de $X$ é mapeado por $f$, de modo que $f^{-1}(Y)=X$, e portanto $\nu(Y)=\mu\left(f^{-1}(Y)\right)=\mu(X)=1$, ou seja, $\nu$ também é uma medida de 
probabilidade. Note ainda, apenas a título de curiosidade, que no caso da função $f:\left(X, \Sigma_{X}\right) \longrightarrow\left(Y, \Sigma_{Y}\right)$ ser injetora, tem-se que $\forall A, A=f^{-1}(f(A))$ de modo que o conhecimento da medida $\nu$ determinará a medida $\mu$ :

$$
\nu(f(A))=\mu\left(f^{-1}(f(A))\right)=\mu(A) .
$$

As triplas $\left(X, \Sigma_{X}, \mu\right)$ e $\left(Y, \Sigma_{Y}, \nu\right)$ são chamadas de espaços de probabilidade. As funções mensuráveis fazem um papel semelhante em relação às $\sigma$-álgebras ao que as funções contínuas fazem em relação às topologias. Enquanto o mapa inverso de uma função mensurável leva elementos de uma $\sigma$-álgebra em elementos de outra, o mapa inverso das funções contínuas leva conjuntos abertos de uma topologia em conjuntos abertos de outra topologia. Desta forma, é simples mostrar que assim como a composição de duas funções contínuas é em sí uma função contínua, a composição de duas funções mensuráveis é em sí uma função mensurável.

Teorema 1.3.3. Sejam $X, Y$ e $Z$ conjuntos quaisquer, e sejam respectivamente $\Sigma_{X}, \Sigma_{Y}$ e $\Sigma_{Z}$ suas $\sigma$-álgebras. Sejam $f: X \longrightarrow Y$ e $g: Y \longrightarrow Z$ duas funções mensuráveis. Então a função composta $f \circ g: X \longrightarrow Z$ é mensurável.

Demonstração. Se $C \in \Sigma_{Z}$, então como $g$ é mensurável, $\exists B \in \Sigma_{Y} \mid B=g^{-1}(C)$. Analogamente, como $f$ é mensurável, então $\exists A \in \Sigma_{X} \mid A=f^{-1}(B)=f^{-1}\left(g^{-1}(C)\right)=$ $(f \circ g)^{-1}(C)$. Portanto, $f \circ g$ é mensurável.

Definição 1.3.4. $\sigma$-álgebra de Borel. Seja $\left(X, \mathcal{T}_{X}\right)$ um espaço topológico. Então pode se mostrar ([39]-1.10) que existe uma menor $\sigma$-álgebra a qual contém todos os conjuntos abertos de $X$. Esta $\sigma$-álgebra é chamada de "conjuntos de Borel de $\mathcal{T}_{X}$ " ou simplesmente de $\operatorname{Borel}\left(\mathcal{T}_{X}\right)$. 
As $\sigma$-álgebras de Borel são extremamente convenientes, pois sua utilização implica imediatamente na mensurabilidade das funções contínuas.

Seja $f:\left(X, \Sigma_{X}, \mu\right) \longrightarrow\left(Y, \Sigma_{Y}\right)$ uma função mensurável. Se $Y=\mathbb{R}$ e $\Sigma_{Y}=$ $\operatorname{Borel}\left(\mathcal{T}_{\mathbb{R}}\right)=\Sigma_{\mathbb{R}}$, então $f$ será chamada de variável aleatória real. Nas próximas seções, um novo tipo de variável aleatória será construída, utilizando como base um conjunto $Y$ diferente dos números reais e que possui a estrutura periódica que está sendo procurada.

Uma das propriedades da $\sigma$-álgebra de Borel dos números reais é que ela é a $\sigma$ álgebra gerada a partir dos intervalos da forma $(-\infty, a)$, que são conjuntos abertos na topologia dos reais. Isto quer dizer que qualquer elemento de $\Sigma_{\mathbb{R}}$ ( evento ) pode ser escrito como união contável de intervalos disjuntos da forma $(-\infty, a)$ e de seus complementos. É possível então definir a função

$$
\begin{aligned}
P_{f}: & \mathbb{R} \longrightarrow \mathbb{R} \\
& a \longmapsto P_{f}(a)=\mu\left(f^{-1}((-\infty, a))\right)=\nu((-\infty, a))
\end{aligned}
$$

Esta função é a conhecida Função Distribuição de Probabilidade da variável aleatória $f$.

A medida de Lebesgue $(\Lambda)$ é a medida utilizada normalmente para se fazer integrais no conjunto dos números reais. Continue supondo então que $Y=\mathbb{R}$ e $\Sigma_{Y}=\Sigma_{\mathbb{R}}$. Uma medida $\nu: \Sigma_{\mathbb{R}} \longrightarrow \mathbb{R}$ pode ser classificada em relação à medida de Lebesgue como absolutamente contínua ou singular. Absolutamente contínua significa que sempre que a medida de Lebesgue for nula, a medida $\nu$ também será nula, isto é $\forall B \in \Sigma_{Y}, \Lambda(B)=0 \Rightarrow \nu(B)=0$. Singular, significa que existem conjuntos em que toda medida $\nu$ se concentra, enquanto nestes mesmos conjuntos a medida de Lebesgue é nula, mais precisamente, $\exists B \in \Sigma_{Y}, \nu(B)=1$ e $\Lambda(B)=0$. De modo geral, 
as medidas podem não ser nem singulares, nem absolutamente contínuas, mas pela decomposição de Lebesgue ([2]-8.11), é sempre possível escrever:

$$
\nu=\alpha \nu_{a c}+(1-\alpha) \nu_{s}, 0 \leqslant \alpha \leqslant 1,
$$

onde com relação à medida de Lebesgue, $\nu_{a c}$ é uma medida absolutamente contínua e $\nu_{s}$ é uma medida singular, e ambas são univocamente determinadas. Seja $M\left(X, \Sigma_{X}\right)$ a coleção de todas as funções reais mensuráveis com domínio $X$ usando a $\sigma$-álgebra $\Sigma_{X}$, e seja $M^{+}\left(X, \Sigma_{X}\right)$ o subconjunto de $M\left(X, \Sigma_{X}\right)$ das funções não-negativas. Então $\nu$ for absolutamente contínua com relação à medida de Lebesgue, então o teorema de Radon-Nikodým ([2]-8.9) diz que existe uma função $p_{f} \in M^{+}\left(X, \Sigma_{X}\right)\left(p_{f}(\alpha) \geqslant 0\right.$, $\forall \alpha)$ tal que:

$$
\nu(B)=\int_{B} p_{f} d \Lambda
$$

A função $p_{f}(\alpha)$ é a conhecida função densidade de probabilidade da variável aleatória $f$, que é também a chamada derivada de Radon-Nikodým de $\nu$ em relação a $\Lambda$. Quando a medida de uma variável aleatória real é singular e concentrada em pontos, é muito comum e conveniente em engenharia e física, escrever que esta variável aleatória possui uma "função densidade de probabilidade" dada por $p_{f}(x)=\sum_{i=0}^{N} \delta_{i}(x)=$ $\sum_{i=0}^{N} \delta\left(x-x_{i}\right)$ de modo que

$$
\nu_{s}(B)=\int_{B} \sum_{i=0}^{N} \delta_{i} d \Lambda .
$$

Tudo se passa como se o teorema de Radon-Nikodým se aplicasse a esse caso. As "funções $\delta "$ de Dirac são uma maneira conveniente de representar um tipo de medida singular em relação à medida de Lebesgue, pois permite representar as integrais das "funções densidade de probabilidade" como uma integral em relação apenas à uma única medida, que é a medida de Lebesgue. Note porém que as "funções $\delta$ " não 
são funções no sentido usual com imagem em $\mathbb{R}$. Na verdade, se consideradas como funções, elas são iguais q.t.p. ( em quase todo ponto, do inglês a.e., almost everywhere ) à função constante e igual a zero. Esse abuso de notação justifica-se pela facilidade de manipulação e compactação de notação das "funções $\delta$ " de Dirac em cálculos relativamente complicados.

\subsection{O Grupo $\left(\mathbb{G}_{\Delta}, \oplus\right)$}

Proposição 1.4.1. Igualdade módulo $\Delta$. Seja $\Delta \in(0,+\infty)$. Defina a relação

$$
\forall x \in \mathbb{R}, \forall y \in \mathbb{R}, x \stackrel{(\bmod \Delta)}{=} y \Leftrightarrow n=\frac{y-x}{\Delta} \in \mathbb{Z} .
$$

A relação assim definida é uma relação de equivalência em $\mathbb{R}$.

Demonstração. Uma relação de equivalência deve satisfazer:

i) Reflexividade $(x \stackrel{(\bmod \Delta)}{=} x)$ :

$$
\begin{gathered}
x \stackrel{(\bmod \Delta)}{=} x \\
\Leftrightarrow \frac{x-x}{\Delta}=0 \in \mathbb{Z}
\end{gathered}
$$

ii) Simetria $(x \stackrel{(\bmod \Delta)}{=} y \Leftrightarrow y \stackrel{(\bmod \Delta)}{=} x)$ :

$$
\begin{aligned}
& x \stackrel{(\bmod \Delta)}{=} y \\
\Leftrightarrow & \frac{y-x}{\Delta} \in \mathbb{Z} \\
\Leftrightarrow & -\frac{y-x}{\Delta} \in \mathbb{Z} \\
\Leftrightarrow & \frac{x-y}{\Delta} \in \mathbb{Z} \\
\Leftrightarrow & y \stackrel{(\bmod \Delta)}{=} x
\end{aligned}
$$


iii) Transitividade $(x \stackrel{(\bmod \Delta)}{=} y, y \stackrel{(\bmod \Delta)}{=} z \Rightarrow x \stackrel{(\bmod \Delta)}{=} z)$ :

$$
\begin{aligned}
& x \stackrel{(\bmod \Delta)}{=} y, y \stackrel{(\bmod \Delta)}{=} z \\
\Leftrightarrow & \frac{y-x}{\Delta} \in \mathbb{Z}, \frac{z-y}{\Delta} \in \mathbb{Z} \\
\Rightarrow & \frac{y-x}{\Delta}+\frac{z-y}{\Delta} \in \mathbb{Z} \\
\Leftrightarrow & \frac{z-x}{\Delta} \in \mathbb{Z} \\
\Leftrightarrow & x \stackrel{(\bmod \Delta)}{=} z
\end{aligned}
$$

Proposição 1.4.2. Se $x, x^{\prime}, y$ e $y^{\prime} \in \mathbb{R}$, e $x \stackrel{(\bmod \Delta)}{=} x^{\prime}$ e $y \stackrel{(\bmod \Delta)}{=} y^{\prime}$, então $x+y \stackrel{(\bmod \Delta)}{=} x^{\prime}+y^{\prime}$.

Demonstração. Pela relação de equivalência definida na proposição 1.4.1,

$$
\left\{\begin{array}{l}
x \stackrel{(\bmod \Delta)}{=} x^{\prime} \Leftrightarrow \exists n=\frac{x^{\prime}-x}{\Delta} \in \mathbb{Z} \\
y \stackrel{(\bmod \Delta)}{=} y^{\prime} \Leftrightarrow \exists m=\frac{y^{\prime}-y}{\Delta} \in \mathbb{Z}
\end{array}\right.
$$

Mas pela mesma definição,

$$
\begin{aligned}
& (x+y) \stackrel{(\bmod \Delta)}{=}\left(x^{\prime}+y^{\prime}\right) \\
\Leftrightarrow & \frac{\left(x^{\prime}+y^{\prime}\right)-(x+y)}{\Delta}=\frac{\left(x^{\prime}-x\right)}{\Delta}-\frac{\left(y^{\prime}-y\right)}{\Delta}=n+m \in \mathbb{Z} .
\end{aligned}
$$

Definição 1.4.3. Conjunto $\mathbb{G}_{\Delta}$. Seja $[x]=\left\{w \in \mathbb{R} \mid \frac{x-w}{\Delta} \in \mathbb{Z}\right\}$ a classe de equivalência relativa ao elemento $x$. Então $\mathbb{G}_{\Delta}$ é o conjunto das classes de equivalência definidas pela relação de equivalência $\stackrel{(\bmod \Delta)}{=}$, isto é, $\mathbb{G}_{\Delta}=\{[x] \mid x \in \mathbb{R}\}$. 
Definição 1.4.4. Soma em $\mathbb{G}_{\Delta}$. Sejam $X, Y \in \mathbb{G}_{\Delta}$, e escolha $x, y \in \mathbb{R}$ de tal forma que $X=[x]$ e $Y=[y]$. Defina então a seguinte operação entre dois elementos de $\mathbb{G}_{\Delta}$ ( duas classes de equivalência):

$$
\begin{aligned}
\oplus: \mathbb{G}_{\Delta} \times \mathbb{G}_{\Delta} & \longrightarrow \mathbb{G}_{\Delta} \\
(X, Y) & \longmapsto[x+y]
\end{aligned}
$$

Pela proposição 1.4.2, a definição de $\oplus$ não depende dos particulares representantes das classes de equivalência $x$ e y escolhidos em $\mathbb{R}$, e portanto, a operação está bem definida.

Lema 1.4.5. O par $\left(\mathbb{G}_{\Delta}, \oplus\right)$ forma um grupo.

Demonstração. Sejam $A, B$ e $C \in \mathbb{G}_{\Delta}$ e $a, b, c \in \mathbb{R}$, tais que $A=[a], B=[b]$ e $C=[c]$. Um grupo deve satisfazer:

G1) Associatividade $\left(A \oplus(B \oplus C)=(A \oplus B) \oplus C, \forall A, B, C \in \mathbb{G}_{\Delta}\right)$ :

$$
\begin{aligned}
& \forall A, B, C \in \mathbb{G}_{\Delta}, \\
& A \oplus(B \oplus C)=A \oplus[b+c]=[a+(b+c)]= \\
& {[(a+b)+c]=[a+b] \oplus C=(A \oplus B) \oplus C}
\end{aligned}
$$

G2) Elemento neutro $\left(\exists 0 \in \mathbb{G}_{\Delta} \mid A \oplus 0=0 \oplus A=A, \forall A \in \mathbb{G}_{\Delta}\right)$ :

$\forall A \in \mathbb{G}_{\Delta}$

i) $A \oplus 0=A \oplus[0]=[a+0]=[0+a]=[0] \oplus[a]=0 \oplus A$

ii) $A \oplus 0=A \oplus[0]=[a+0]=[a]=A$ 
G3) Existência da inversa $\left(\forall A \in \mathbb{G}_{\Delta}, \exists B \in \mathbb{G}_{\Delta} \mid A \oplus B=B \oplus A=0\right)$ :

$$
\begin{aligned}
& \forall A, B \in \mathbb{G}_{\Delta}, \\
& 0=[0]=[a+((-a)+\Delta \cdot n)]= \\
& {[a]+[(-a)+\Delta \cdot n]=A \oplus[(-a)]=A \oplus B=A \oplus(-A)}
\end{aligned}
$$

O grupo assim definido satisfaz também a seguinte propriedade:

$$
\text { G4) } \forall A, B \in \mathbb{G}_{\Delta}, A \oplus B=B \oplus A
$$

o que o torna um grupo comutativo ou abeliano. No desenvolvimento a seguir, não é necessário que o grupo utilizado seja abeliano. Note que ficou mostrado também que a classe de equivalência do elemento neutro da propriedade G2) é derivada dos elementos de $\mathbb{R}$ da forma $0+n \Delta, n \in \mathbb{Z}$.

A propriedade G3) permite definir a operação $\ominus$, inversa de $\oplus$, da forma usual:

Definição 1.4.6. Subtração em $\mathbb{G}_{\Delta}$. Sejam $X, Y \in \mathbb{G}_{\Delta}$. Defina então a seguinte operação:

$$
\begin{aligned}
\ominus: \mathbb{G}_{\Delta} \times \mathbb{G}_{\Delta} & \longrightarrow \mathbb{G}_{\Delta} \\
(X, Y) & \longmapsto Z=X \oplus(-Y)
\end{aligned}
$$

Seja $I_{[a, \Delta]}=\{x \in \mathbb{R} \mid a \leqslant x<a+\Delta\}$. Uma vez escolhido o número real $a$, é possível definir uma função inversível $\Pi_{[a, \Delta]}: \mathbb{G}_{\Delta} \longrightarrow I_{[a, \Delta]}$ de tal modo que $\forall B \in \mathbb{G}_{\Delta}$, $\left\{\Pi_{[a, \Delta]}(B)\right\}=B \cap I_{[a, \Delta]}=\{b\}$, onde obviamente $B=[b]$. Através desta função e de sua inversa, é possível transportar qualquer estrutura matemática entre os conjuntos $\mathbb{G}_{\Delta}$ e $I_{[a, \Delta]}$. Pode-se, por exemplo, transportar a ordenação parcial " $\leqslant$ " dos números 
reais para $\mathbb{G}_{\Delta}$, bastando para isso escolher um $I_{[a, \Delta]}$ para fazer a comparação. Note que o resultado da comparação depende do valor $a$ escolhido. A partir do momento em que $a$ for escolhido, $\inf _{x \in \mathbb{G}_{\Delta}} \mathbb{G}_{\Delta}=[a]$. Além disso, $\nexists w \in \mathbb{G}_{\Delta} \mid w=\sup _{x \in \mathbb{G}_{\Delta}} \mathbb{G}_{\Delta}$. Na prática, a relação de ordem é apenas uma maneira conveniente de se especificar subconjuntos de $\mathbb{G}_{\Delta}$ por analogia a $I_{[a, \Delta]}$, uma vez que a simetria circular do conjunto $\mathbb{G}_{\Delta}$ impede que uma relação de ordem seja criada sem ao mesmo tempo quebrar a simetria. Em termos angulares, não faz sentido dizer que um determinado ângulo é maior ou menor que outro. A escolha do número a executa esta quebra de simetria.

A proposição 1.4.12 ensina a calcular o representante de uma classe de equivalência que cai num determinado intervalo, isto é, qual é o $y \in I_{[a, \Delta]} \mid \forall x \in \mathbb{R}, y \stackrel{(\bmod \Delta)}{=} x$. Para isto, as funções de arredondamento " $\lfloor x\rfloor " \mathrm{e}$ " $\lceil x\rceil$ " serão introduzidas. O símbolo

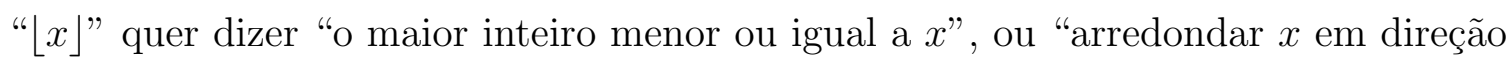
a menos infinito", ou ainda "o chão de $x$ ". O símbolo " $\lceil x\rceil$ " quer dizer "o menor inteiro maior ou igual a $x$ ", ou "arredondar $x$ em direção a mais infinito", ou ainda "o teto de $x$ ". É interessante introduzir também a notação da função característica de um conjunto.

Definição 1.4.7. Função característica de um conjunto A.

$$
\chi_{A}(x)=\left\{\begin{array}{l}
0, \text { se } x \notin A \\
1, \text { se } x \in A
\end{array} .\right.
$$

Definição 1.4.8. Função chão.

$$
\begin{aligned}
\lfloor x\rfloor: \mathbb{R} & \longrightarrow \mathbb{Z} \\
x & \longmapsto \max _{w \in \mathbb{Z}}\{w: w \leqslant x\}
\end{aligned}
$$


Definição 1.4.9. Função teto.

$$
\begin{aligned}
\lceil x\rceil: \mathbb{R} & \longrightarrow \mathbb{Z} \\
x & \longmapsto \min _{w \in \mathbb{Z}}\{w: w \geqslant x\}
\end{aligned}
$$

Definição 1.4.10. Função "parte fracionária".

$$
\begin{aligned}
\operatorname{Fr}(x): \mathbb{R} & \longrightarrow \mathbb{R} \\
x & \longmapsto x-\lfloor x\rfloor
\end{aligned}
$$

Proposição 1.4.11. As funções de arredondamento possuem as seguintes propriedades que serão utilizadas em demonstrações mais adiante:

i) $\lfloor a\rfloor \leqslant a \leqslant\lceil a\rceil$

ii) $0 \leqslant \operatorname{Fr}(a)<1$

iii) $\lfloor a\rfloor \leqslant a<\lfloor a\rfloor+1$

iv) $\lfloor a+k\rfloor=\lfloor a\rfloor+k \Leftrightarrow k \in \mathbb{Z}$

v) $\lceil a+k\rceil=\lceil a\rceil+k \Leftrightarrow k \in \mathbb{Z}$

vi) As funções $\lfloor x\rfloor e\lceil x\rceil$ são monótonas crescentes.

vii) $a \in \mathbb{Z} \Leftrightarrow\lfloor a\rfloor=a=\lceil a\rceil \Leftrightarrow \operatorname{Fr}(a)=0$

viii) $\lfloor-a\rfloor=-\lceil a\rceil$ ou de modo equivalente $\lceil-a\rceil=-\lfloor a\rfloor$

ix) $\begin{cases}\forall a, b \in \mathbb{R} & \Rightarrow 0 \leqslant\lfloor\operatorname{Fr}(a)+\operatorname{Fr}(b)\rfloor \leqslant 1 \\ a \in \mathbb{Z} \text { ou } b \in \mathbb{Z} & \Rightarrow\lfloor\operatorname{Fr}(a)+\operatorname{Fr}(b)\rfloor=0\end{cases}$ 
x) $a=\operatorname{Fr}(a) \Leftrightarrow 0 \leqslant a<1$

xi) $\lceil a\rceil-\lfloor a\rfloor=\left\{\begin{array}{l}0, \quad \text { se } a \in \mathbb{Z} \\ 1, \quad \text { se } a \notin \mathbb{Z}\end{array} \Longleftrightarrow 1-\lceil a\rceil+\lfloor a\rfloor=\chi_{\mathbb{Z}}(a)\right.$

xii) $\operatorname{Fr}(a)+\operatorname{Fr}(-a)=\lceil a\rceil-\lfloor a\rfloor=1-\chi_{\mathbb{Z}}(a)$

xiii) $\lfloor a+b\rfloor=\lfloor a\rfloor+\lfloor b\rfloor+\lfloor\operatorname{Fr}(a)+\operatorname{Fr}(b)\rfloor$

xiv) $\lceil a+b\rceil=\lfloor a\rfloor+\lfloor b\rfloor+\lceil\operatorname{Fr}(a)+\operatorname{Fr}(b)\rceil=\lceil a\rceil+\lceil b\rceil-\lfloor\operatorname{Fr}(-a)+\operatorname{Fr}(-b)\rfloor$

Demonstração.

i) $\lfloor a\rfloor \leqslant a \leqslant\lceil a\rceil$

Pela definição 1.4.8, $\lfloor a\rfloor \in\{w \in \mathbb{Z}: w \leqslant a\}$, logo $\lfloor a\rfloor \leqslant a$. Analogamente, pela definição 1.4.9, $\lceil a\rceil \in\{w \in \mathbb{Z}: w \geqslant a\}, \operatorname{logo}\lceil a\rceil \geqslant a$. Portanto, $\lfloor a\rfloor \leqslant a \leqslant\lceil a\rceil$.

ii) $0 \leqslant \operatorname{Fr}(a)<1$

Pelo item i), $\lfloor a\rfloor \leqslant a \Leftrightarrow a-\lfloor a\rfloor \geqslant 0 \Leftrightarrow \operatorname{Fr}(a) \geqslant 0$. Para mostrar que $\operatorname{Fr}(a)<1$, assuma que $\operatorname{Fr}(a) \geqslant 1$. Então $a-\lfloor a\rfloor \geqslant 1 \Leftrightarrow\lfloor a\rfloor+1 \leqslant a$. Mas isto entra em contradição com a definição original da função chão, pois $\lfloor a\rfloor+1 \geqslant\lfloor a\rfloor$, $\lfloor a\rfloor+1 \in \mathbb{Z}$ e $\lfloor a\rfloor=\max _{w \in \mathbb{Z}} w: w \leqslant a$. Portanto, tem-se que ter $\lfloor a\rfloor<1$.

iii) $\lfloor a\rfloor \leqslant a<\lfloor a\rfloor+1$

$$
\begin{aligned}
0 \leqslant \operatorname{Fr}(a)<1 & \Leftrightarrow\lfloor a\rfloor \leqslant\lfloor a\rfloor+\operatorname{Fr}(a)<\lfloor a\rfloor+1 \\
& \Leftrightarrow\lfloor a\rfloor \leqslant a<\lfloor a\rfloor+1
\end{aligned}
$$

iv) $\lfloor a+k\rfloor=\lfloor a\rfloor+k \Leftrightarrow k \in \mathbb{Z}$

$$
\lfloor a+k\rfloor=\lfloor a\rfloor+k \Leftrightarrow \max _{w \in \mathbb{Z}}\{w: w \leqslant a+k\}=\max _{w \in \mathbb{Z}}\{w: w \leqslant a\}+k
$$




$$
\begin{aligned}
& \Leftrightarrow \max _{w \in \mathbb{Z}}\{w: w \leqslant a+k\}=\max _{w \in \mathbb{Z}}\{w+k: w \leqslant a\} \\
(v=w+k) & \Leftrightarrow \max _{w \in \mathbb{Z}}\{w: w \leqslant a+k\}=\max _{v-k \in \mathbb{Z}}\{v: v-k \leqslant a\} \\
& \Leftrightarrow \max _{w \in \mathbb{Z}}\{w: w \leqslant a+k\}=\max _{v \in \mathbb{Z}+k}\{v: v \leqslant a+k\} \\
& \Leftrightarrow \mathbb{Z}=\mathbb{Z}+k \\
& \Leftrightarrow k \in \mathbb{Z}
\end{aligned}
$$

v) $\lceil a+k\rceil=\lceil a\rceil+k \Leftrightarrow k \in \mathbb{Z}$

A demonstração é inteiramente análoga à do item anterior.

vi) As funções $\lfloor x\rfloor$ e $\lceil x\rceil$ são monótonas crescentes.

$$
\begin{aligned}
b \geqslant a \Rightarrow\lfloor b\rfloor & =\max _{w \in \mathbb{Z}}\{w: w \leqslant b\} \\
& =\max _{w \in \mathbb{Z}}[\{w: w \leqslant a\} \cup\{w: a<w \leqslant b\}] \\
& \geqslant \max _{w \in \mathbb{Z}}\{w: w \leqslant a\}=\lfloor a\rfloor
\end{aligned}
$$

Analogamente,

$$
\begin{aligned}
a \leqslant b \Rightarrow\lceil a\rceil & =\min _{w \in \mathbb{Z}}\{w: w \geqslant a\} \\
& =\min _{w \in \mathbb{Z}}[\{w: a \leqslant w<b\} \cup\{w: w \geqslant b\}] \\
& \leqslant \min _{w \in \mathbb{Z}}\{w: w \geqslant b\}=\lceil b\rceil
\end{aligned}
$$

vii) $a \in \mathbb{Z} \Leftrightarrow\lfloor a\rfloor=a=\lceil a\rceil \Leftrightarrow \operatorname{Fr}(a)=0$

As definições 1.4 .8 e 1.4 .9 implicam trivialmente que se $a \in \mathbb{Z} \Rightarrow\lfloor a\rfloor=a=\lceil a\rceil$.

Por outro lado, se $\lfloor a\rfloor=\lceil a\rceil=b \in \mathbb{Z}$, então pelo item i) $b \leqslant a$ e $a \leqslant b \Rightarrow a=$ $b \in \mathbb{Z}$. Finalmente $\lfloor a\rfloor=a \Leftrightarrow\lfloor a\rfloor-a=0 \Leftrightarrow \operatorname{Fr}(a)=0$.

viii) $\lfloor-a\rfloor=-\lceil a\rceil$ ou de modo equivalente $\lceil-a\rceil=-\lfloor a\rfloor$

$$
\lfloor-a\rfloor=\max _{w \in \mathbb{Z}}\{w: w \leqslant-a\}
$$




$$
\begin{aligned}
& =\max _{w \in \mathbb{Z}}\{w:-w \geqslant a\} \\
(v=-w) & =\max _{-v \in \mathbb{Z}}\{-v: v \geqslant a\} \\
& =-\min _{v \in \mathbb{Z}}\{v: v \geqslant a\} \\
& =-\lceil a\rceil .
\end{aligned}
$$

Basta então trocar $a$ por $-a$ para obter $\lceil-a\rceil=-\lfloor a\rfloor$.

ix) $\begin{cases}\forall a, b \in \mathbb{R} & \Rightarrow 0 \leqslant\lfloor\operatorname{Fr}(a)+\operatorname{Fr}(b)\rfloor \leqslant 1 \\ a \in \mathbb{Z} \text { ou } b \in \mathbb{Z} & \Rightarrow\lfloor\operatorname{Fr}(a)+\operatorname{Fr}(b)\rfloor=0\end{cases}$

Usando o item i) duas vezes,

$$
\left\{\begin{aligned}
0 \leqslant \operatorname{Fr}(a)<1 & \Rightarrow 0 \leqslant \operatorname{Fr}(a)+\operatorname{Fr}(b)<2 \\
0 \leqslant \operatorname{Fr}(b)<1 & \Rightarrow 0 \leqslant\lfloor\operatorname{Fr}(a)+\operatorname{Fr}(b)\rfloor<2 \\
(\lfloor x\rfloor \in \mathbb{Z}) & \Rightarrow 0 \leqslant\lfloor\operatorname{Fr}(a)+\operatorname{Fr}(b)\rfloor \leqslant 1
\end{aligned}\right.
$$

Para o segundo caso, suponha sem perda de generalidade que $a \in \mathbb{Z}$. Então,

$$
\begin{aligned}
a \in \mathbb{Z} & \Leftrightarrow \operatorname{Fr}(a)=0 \\
& \Rightarrow\lfloor\operatorname{Fr}(a)+\operatorname{Fr}(b)\rfloor=\lfloor\operatorname{Fr}(b)\rfloor= \\
{[\text { pelo item ii })] } & =0
\end{aligned}
$$

x) $a=\operatorname{Fr}(a) \Leftrightarrow 0 \leqslant a<1$

$$
\begin{aligned}
a=\operatorname{Fr}(a) & \Leftrightarrow a-\operatorname{Fr}(a)=0 \\
& \Leftrightarrow a-(a-\lfloor a\rfloor)=0 \\
& \Leftrightarrow\lfloor a\rfloor=0
\end{aligned}
$$




$$
\begin{aligned}
{[\text { pelo item iii) }] } & \Leftrightarrow\lfloor 0\rfloor \leqslant a<\lfloor 0\rfloor+1 \\
& \Leftrightarrow 0 \leqslant a<1
\end{aligned}
$$

xi) $\lceil a\rceil-\lfloor a\rfloor=\left\{\begin{array}{ll}0, & \text { se } a \in \mathbb{Z} \\ 1, & \text { se } a \notin \mathbb{Z}\end{array}=1-\chi_{\mathbb{Z}}(a)\right.$

Tem-se que $\lceil a\rceil-\lfloor a\rfloor=\lceil\lfloor a\rfloor+\operatorname{Fr}(a)\rceil-\lfloor a\rfloor=\lceil\operatorname{Fr}(a)\rceil+\lfloor a\rfloor-\lfloor a\rfloor=\lceil\operatorname{Fr}(a)\rceil$.

Pelo item vii), $a \in \mathbb{Z} \Leftrightarrow \operatorname{Fr}(a)=0$, logo se $a \in \mathbb{Z} \Leftrightarrow\lceil a\rceil-\lfloor a\rfloor=0$. Mas se $a \notin \mathbb{Z}$, então $0<\operatorname{Fr}(a)<1 \Rightarrow\lceil\operatorname{Fr}(a)\rceil=1$. A segunda parte da relação é apenas uma maneira de escrever a relação usando a função característica.

xii) $\operatorname{Fr}(a)+\operatorname{Fr}(-a)=\lceil a\rceil-\lfloor a\rfloor=1-\chi_{\mathbb{Z}}(a)$

$$
\left\{\begin{array}{l}
\operatorname{Fr}(a)=a-\lfloor a\rfloor \\
\operatorname{Fr}(-a)=-a-\lfloor-a\rfloor
\end{array} \Rightarrow \operatorname{Fr}(a)+\operatorname{Fr}(-a)=-\lfloor-a\rfloor-\lfloor a\rfloor=\lceil a\rceil-\lfloor a\rfloor .\right.
$$

A segunda parte da igualdade decorre imediatamente do item xi).

xiii) $\lfloor a+b\rfloor=\lfloor a\rfloor+\lfloor b\rfloor+\lfloor\operatorname{Fr}(a)+\operatorname{Fr}(b)\rfloor$

$$
\begin{aligned}
\lfloor a+b\rfloor & =\lfloor\lfloor a\rfloor+\operatorname{Fr}(a)+\lfloor b\rfloor+\operatorname{Fr}(b)\rfloor \\
{[\text { pelo item iv })] } & =\lfloor a\rfloor+\lfloor b\rfloor+\lfloor\operatorname{Fr}(a)+\operatorname{Fr}(b)\rfloor
\end{aligned}
$$

xiv) $\lceil a+b\rceil=\lfloor a\rfloor+\lfloor b\rfloor+\lceil\operatorname{Fr}(a)+\operatorname{Fr}(b)\rceil=\lceil a\rceil+\lceil b\rceil-\lfloor\operatorname{Fr}(-a)+\operatorname{Fr}(-b)\rfloor$

$$
\begin{aligned}
\lceil a+b\rceil & =\lceil\lfloor a\rfloor+\operatorname{Fr}(a)+\lfloor b\rfloor+\operatorname{Fr}(b)\rceil \\
{[\text { pelo item v })] } & =\lfloor a\rfloor+\lfloor b\rfloor+\lceil\operatorname{Fr}(a)+\operatorname{Fr}(b)\rceil \\
{[\text { pelo item xii })] } & =\lfloor a\rfloor+\lfloor b\rfloor+\lceil\lceil a\rceil-\lfloor a\rfloor-\operatorname{Fr}(-a)+\lceil b\rceil-\lfloor b\rfloor-\operatorname{Fr}(-b)\rceil \\
{[\text { pelo item v) }] } & =\lceil a\rceil+\lceil b\rceil+\lceil-\operatorname{Fr}(-a)-\operatorname{Fr}(-b)\rceil \\
{[\text { pelo item viii })] } & =\lceil a\rceil+\lceil b\rceil-\lfloor\operatorname{Fr}(-a)+\operatorname{Fr}(-b)\rfloor
\end{aligned}
$$


Proposição 1.4.12. Representante de uma classe de equivalência no intervalo $I_{[a, \Delta]}$. Seja $x \in \mathbb{R}$. Se $y=\left\lceil\frac{(a-x)}{\Delta}\right\rceil \Delta+x$, então:
i) $y \stackrel{(\bmod \Delta)}{=} x$
ii) $y \in I_{[a, \Delta]}$.

Demonstração.

$$
\begin{aligned}
& y \stackrel{(\bmod \Delta)}{=} x \\
(\text { proposição 1.4.1) } & \Leftrightarrow \frac{y-x}{\Delta}=n \in \mathbb{Z} \\
& \Leftrightarrow y=n \Delta+x, n \in \mathbb{Z}
\end{aligned}
$$

Como $\left\lceil\frac{(a-x)}{\Delta}\right\rceil$ é um número inteiro, tomando $n=\left\lceil\frac{(a-x)}{\Delta}\right\rceil$ a primeira parte está provada. Tem-se agora que mostrar que $n=\left\lceil\frac{(a-x)}{\Delta}\right\rceil$ que faz com que $y \in I_{[a, \Delta]}$ :

$$
\begin{aligned}
& y \in I_{[a, \Delta]} \\
\Leftrightarrow & a \leqslant y<a+\Delta \\
\Leftrightarrow & a \leqslant n \Delta+x<a+\Delta \\
\Leftrightarrow & a-x \leqslant n \Delta<a-x+\Delta \\
\Leftrightarrow & \frac{a-x}{\Delta} \leqslant n<\frac{a-x}{\Delta}+1 \\
\Leftrightarrow & n=\left\lceil\frac{a-x}{\Delta}\right]
\end{aligned}
$$

Definição 1.4.13. Definição dos mapeamentos $\Pi_{[a, \Delta]}$ e $\Pi_{[a, \Delta]}^{-1}$. 
Demonstração. Seja a seguinte função:

$$
\begin{aligned}
\Pi_{[a, \Delta]}: \mathbb{G}_{\Delta} & \longrightarrow I_{[a, \Delta]} \\
X & \longmapsto x \mid x=X \cap I_{[a, \Delta]} \\
\text { e sua inversa } & \\
\Pi_{[a, \Delta]}^{-1}: I_{[a, \Delta]} & \longrightarrow \mathbb{G}_{\Delta} \\
x & \longmapsto X=[x] .
\end{aligned}
$$

Primeiramente, mostra-se que $\Pi_{[a, \Delta]}$ e $\Pi_{[a, \Delta]}^{-1}$ estão bem definidas e são realmente funções, isto é, $\forall X \in \mathbb{G}_{\Delta}, \exists ! x \in I_{[a, \Delta]} \mid x=\Pi_{[a, \Delta]}(X)$ e $\forall x \in I_{[a, \Delta]}, \exists ! X \in \mathbb{G}_{\Delta} \mid x=$ $\Pi_{[a, \Delta]}^{-1}(X)$.

$$
\begin{aligned}
& X \in \mathbb{G}_{\Delta} \Leftrightarrow \exists x_{0} \in \mathbb{R} \mid X=\left\{x \in \mathbb{R} \mid \frac{x-x_{0}}{\Delta}=n, n \in \mathbb{Z}\right\} \\
\Leftrightarrow & x \in X \Leftrightarrow x=x_{0}+n \Delta, n \in \mathbb{Z}
\end{aligned}
$$

e

$$
\begin{gathered}
x \in I_{[a, \Delta]} \\
\Leftrightarrow \quad a \leqslant x<a+\Delta
\end{gathered}
$$

Então: $\quad x \in X \cap I_{[a, \Delta]} \Leftrightarrow x \in X$ e $x \in I_{[a, \Delta]}$

$\Leftrightarrow a \leqslant x<a+\Delta$

$$
\begin{aligned}
\text { (prop.1.4.12) } \Leftrightarrow & x=x_{0}+\left\lceil\frac{a-x_{0}}{\Delta}\right\rceil \Delta \\
\text { portanto, } & \quad x \in X \cap I_{[a, \Delta]} \Leftrightarrow x=x_{0}+\left\lceil\frac{a-x_{0}}{\Delta}\right\rceil \Delta
\end{aligned}
$$

Para mostrar que $\forall x \in I_{[a, \Delta]}, \exists ! X \in \mathbb{G}_{\Delta} \mid x=\Pi_{[a, \Delta]}^{-1}(X)$, basta notar que as classes de equivalência geradas pela relação de equivalência são todas distintas. Em segundo lugar, mostra-se que $\Pi_{[a, \Delta]}$ e $\Pi_{[a, \Delta]}^{-1}$ são mapeamentos injetores: 


$$
\begin{aligned}
& \forall X, Y \in \mathbb{G}_{\Delta}, \Pi_{[a, \Delta]}(X)=\Pi_{[a, \Delta]}(Y) \Rightarrow X=Y \\
\text { Pela primeira parte, } \quad & x=\Pi_{[a, \Delta]}(X)=x_{0}+\left\lceil\frac{a-x_{0}}{\Delta}\right\rceil \Delta \\
& y=\Pi_{[a, \Delta]}(Y)=y_{0}+\left\lceil\frac{a-y_{0}}{\Delta}\right\rceil \Delta \\
\text { portanto, } \quad & x=y \\
\Leftrightarrow & x_{0}+\left\lceil\frac{a-x_{0}}{\Delta}\right\rceil \Delta=y_{0}+\left\lceil\frac{a-y_{0}}{\Delta}\right\rceil \Delta \\
\Leftrightarrow & \frac{x_{0}-y_{0}}{\Delta}=\left\lceil\frac{a-y_{0}}{\Delta}\right\rceil-\left\lceil\frac{a-x_{0}}{\Delta}\right\rceil=m \in \mathbb{Z} \\
\Leftrightarrow & {\left[x_{0}\right]=\left[y_{0}\right] } \\
\Leftrightarrow & X=Y
\end{aligned}
$$

provando também que $\quad \forall x, y \in I_{[a, \Delta]}, \Pi_{[a, \Delta]}^{-1}(X)=\Pi_{[a, \Delta]}^{-1}(Y) \Rightarrow X=Y$

Finalmente, mostra-se que $\Pi_{[a, \Delta]}$ e $\Pi_{[a, \Delta]}^{-1}$ são mapeamentos sobrejetores:

$$
\begin{aligned}
\text { Para mostrar que } & \forall x \in I_{[a, \Delta]}, \exists X \in \mathbb{G}_{\Delta} \mid x=\prod_{[a, \Delta]}(X) \\
\text { basta fazer } & X=\{y \mid y=x+n \Delta, \Delta \in \mathbb{Z}\}, \\
\text { e para mostrar que } & \forall X \in \mathbb{G}_{\Delta}, \exists x \in I_{[a, \Delta]} \mid X=\left[x_{0}\right]=\Pi_{[a, \Delta]}^{-1}(x) \\
\text { basta fazer } & x=x_{0}+\left\lceil\frac{a-x_{0}}{\Delta}\right\rceil \Delta .
\end{aligned}
$$

As seguintes funções serão úteis mais adiante.

Definição 1.4.14. Função $\bmod (x, \Delta)$. Sejam $x \in \mathbb{R} e \Delta \in \mathbb{R}, \Delta>0$. Defina então a função:

$$
\begin{aligned}
\bmod : \mathbb{R} \times(0,+\infty) & \longrightarrow I_{[0, \Delta]} \\
(x, \Delta) & \longmapsto y=x-\left\lfloor\frac{x}{\Delta}\right\rfloor \times \Delta .
\end{aligned}
$$


Isto é, a função $\bmod (x, \Delta)$ tem como valor o resto da divisão inteira de $x$ por $\Delta$.

Definição 1.4.15. Função $\bmod 2(x, \Delta, a)$. Sejam $x \in \mathbb{R}, \Delta \in \mathbb{R}, \Delta>0$ e a $\in \mathbb{R}$. Defina então a função:

$$
\begin{aligned}
\bmod 2: \mathbb{R} \times(0,+\infty) \times \mathbb{R} & \longrightarrow I_{[a, \Delta]} \\
(x, \Delta, a) & \longmapsto y=x+\left\lceil\frac{a-x}{\Delta}\right\rceil \times \Delta=x-\left\lfloor\frac{x-a}{\Delta}\right\rfloor \times \Delta .
\end{aligned}
$$

Portanto, $\bmod 2(x, \Delta, 0)=\bmod (x, \Delta)$, ou seja, a função $\bmod 2(x, \Delta, a)$ é uma generalização da tradicional função $\bmod (x, \Delta)$.

Proposição 1.4.16. Operação $\oplus$ em $I_{[a, \Delta]}$. Defina a seguinte operação:

$$
\begin{aligned}
\oplus: I_{[a, \Delta]} \times I_{[a, \Delta]} & \longrightarrow I_{[a, \Delta]} \\
(x, y) & \longmapsto z=\bmod 2(x+y, \Delta, a) .
\end{aligned}
$$

Então vale a seguinte expressão, que justifica este abuso de notação da operação $\oplus$ :

$$
[x] \oplus[y]=[x \oplus y] .
$$

Demonstração.

$$
\begin{aligned}
& {[x] \oplus[y] } \\
(\text { Def.1.4.4) }= & {[x+y] } \\
= & {\left[x+y+\left\lceil\frac{a-x-y}{\Delta}\right\rceil \Delta\right] } \\
= & {[\bmod 2(x+y, \Delta, a)] } \\
= & {[x \oplus y] }
\end{aligned}
$$


Proposição 1.4.17. Operação $\ominus$ em $I_{[a, \Delta]}$. Defina a seguinte operação:

$$
\begin{aligned}
\ominus: I_{[a, \Delta]} \times I_{[a, \Delta]} & \longrightarrow I_{[a, \Delta]} \\
(x, y) & \longmapsto z=\bmod 2(x-y, \Delta, a) .
\end{aligned}
$$

Então vale a seguinte expressão, que justifica este abuso de notação da operação $\oplus$ :

$$
[x] \ominus[y]=[x \ominus y]
$$

Demonstração.

$$
\begin{aligned}
& {[x] \ominus[y] } \\
& {[x] \oplus(-[y]) } \\
= & {[x+\Delta-\bmod 2(y, \Delta, a)] } \\
= & {\left[x+\Delta-y-\left[\frac{a+y}{\Delta}\right\rceil \Delta\right] } \\
= & {[x-y] } \\
= & {[\bmod 2(x-y, \Delta, a)] } \\
= & {[x \ominus y] }
\end{aligned}
$$

\subsection{Topologia em $\mathbb{G}_{\Delta}$}

A topologia é uma estrutura desejável em $\mathbb{G}_{\Delta}$ que não pode ser transportada diretamente a partir da topologia usual de $I_{[a, \Delta]}$. Topologia é, por definição, o conjunto dos conjuntos abertos, e ela é uma noção que antecede o conceito de métrica, que é a regra com a qual se atribuem as distâncias entre os elementos de um conjunto. No 
entanto, ao se especificar uma métrica, automaticamente se escolhe uma topologia, de modo que é possível falar da topologia induzida por uma métrica em particular. Em outras palavras, toda métrica induz uma topologia, mas nem toda topologia é metrizável.

A estrutura cíclica desejada em $\left(\mathbb{G}_{\Delta}, \oplus\right)$ exige que a topologia deste conjunto seja diferente daquela com que usualmente se trabalha nos números reais. Mais especificamente, seria desejável que a operação $\oplus$ definida em $\mathbb{G}_{\Delta}$ fosse contínua, o que não é verdade na métrica usual de $I_{[a, \Delta]}$. A fim de se especificar a topologia de $\mathbb{G}_{\Delta}$, uma nova métrica será criada em $I_{[a, \Delta]}$, de modo a induzir a topologia desejada tanto em $I_{[a, \Delta]}$ quanto em $\mathbb{G}_{\Delta}$ através do mapeamento $\Pi_{[a, \Delta]}$.

Como motivação para o assunto desta seção, considere o problema de se atribuir uma distância entre dois pontos de um círculo, contando essa distância sobre o arco. Dados dois pontos de um círculo, existem dois caminhos possíveis para se sair de um deles e se chegar ao outro: o sentido trigonométrico ou o sentido horário. Considere um círculo de raio unitário e escolha um ponto para chamar de 0. Deste modo podem ser atribuidos aos pontos sobre o círculo ângulos que variam entre 0 e $2 \pi$. No caso do caminho que não passe pelo ponto 0 , a distância entre dois pontos será o valor absoluto da diferença entre o valor dos ângulos aos quais os pontos sobre o círculo correspondem, ou seja, se $\alpha$ é o ângulo do primeiro ponto $\left(P_{1}\right)$ e $\beta$ é o ângulo do segundo ponto $\left(P_{2}\right)$, então $d_{1}\left(P_{1}, P_{2}\right)=|\alpha-\beta|$. No caso do sentido que contenha o ponto 0 , a distância entre os dois pontos será $d_{2}\left(P_{1}, P_{2}\right)=2 \pi-|\alpha-\beta|$. Claramente, se $P_{1}$ está "perto" de $P_{2}$ no sentido da métrica $d_{1}$, ele estará "longe" no sentido da função $d_{2}$, e vice-versa. A noção de distância que está sendo procurada, não deve depender da particular escolha do ponto 0 sobre o círculo. 
Proposição 1.5.1. Métrica usual em $I_{[a, \Delta]}$. Seja a seguinte função:

$$
\begin{aligned}
d: I_{[a, \Delta]} \times I_{[a, \Delta]} & \longrightarrow \mathbb{R} \\
(x, y) & \longmapsto z=|x-y| .
\end{aligned}
$$

Então, a função d acima definida é uma métrica em $I_{[a, \Delta]}$ e será chamada de métrica usual de $I_{[a, \Delta]}$.

Demonstração. A função $d$ acima definida nada mais é do que a restrição da métrica usual do conjunto $\mathbb{R}$ dos números reais ao conjunto $I_{[a, \Delta]}$, que é um subconjunto de $\mathbb{R}$, isto é, o par $\left(I_{[a, \Delta]}, d\right)$ é um sub-espaço métrico de $(\mathbb{R}, d)([32]-3.4)$.

Definição 1.5.2. A topologia induzida pela métrica d em $I_{[a, \Delta]}$ será denotada por $\mathcal{T}_{I_{[a, \Delta]}}$.

Proposição 1.5.3. Limitante da Métrica usual em $I_{[a, \Delta]}$. Sejam $x, y \in I_{[a, \Delta]}$. Então, vale a seguinte relação:

$$
\forall x, y \in I_{[a, \Delta]}, d(x, y)<\Delta
$$

Demonstração. Pela definição de $I_{[a, \Delta]}$, tem-se que

$$
\left\{\begin{array}{l}
x \in I_{[a, \Delta]} \Leftrightarrow a \leqslant x<a+\Delta \\
y \in I_{[a, \Delta]} \Leftrightarrow a \leqslant y<a+\Delta .
\end{array}\right.
$$

As desigualdades acima implicam em:

$$
\left\{\begin{array}{l}
x-a \geqslant 0 \\
a+\Delta-y>0
\end{array} \Rightarrow \Delta+x-y>0 \Rightarrow \Delta>y-x\right.
$$




$$
\left\{\begin{array}{l}
y-a \geqslant 0 \\
a+\Delta-x>0
\end{array} \Rightarrow \Delta+y-x>0 \Rightarrow \Delta>x-y\right.
$$

O que significa que $\Delta>|x-y|=d(x, y)$.

Proposição 1.5.4. Desigualdade Triangular Complementar em $I_{[a, \Delta]}$. Sejam x, y, $z \in I_{[a, \Delta]}$. Então, vale a seguinte relação:

$$
d(x, y)<[\Delta-d(x, z)]+[\Delta-d(z, y)]
$$

A desigualdade acima será chamada de Desigualdade Triangular Complementar.

Demonstração. Para mostrar essa desigualdade, devem ser analisados seis casos, que se reduzem a três porque a expressão é simétrica em relação à troca de $x$ por $y$. Note que o que será mostrado a seguir é que em cada caso a Desigualdade Triangular Complementar é equivalente à proposição 1.5.3 ( limitação da métrica em $I_{[a, \Delta]}$ ):

i) $x \leqslant y \leqslant z:$

$$
\begin{aligned}
& |x-y|<2 \Delta-|x-z|-|y-z| \\
\Leftrightarrow & y-x<2 \Delta-(z-x)-(z-y) \\
\Leftrightarrow & y-x<2 \Delta-z+x-z+y \\
\Leftrightarrow & -2 x<2 \Delta-2 z \\
\Leftrightarrow & z-x<\Delta \\
\Leftrightarrow & |z-x|<\Delta \\
\Leftrightarrow & d(z, x)<\Delta
\end{aligned}
$$

ii) $x \leqslant z \leqslant y$ :

$$
|x-y|<2 \Delta-|x-z|-|y-z|
$$




$$
\begin{aligned}
& \Leftrightarrow \quad y-x<2 \Delta-(z-x)-(y-z) \\
& \Leftrightarrow \quad y-x<2 \Delta-z+x-y+z \\
& \Leftrightarrow \quad 2 y-2 x<2 \Delta \\
& \Leftrightarrow y-x<\Delta \\
& \Leftrightarrow \quad|y-x|<\Delta \\
& \Leftrightarrow d(y, x)<\Delta
\end{aligned}
$$

iii) $z \leqslant x \leqslant y$ :

$$
\begin{aligned}
& |x-y|<2 \Delta-|x-z|-|y-z| \\
\Leftrightarrow & y-x<2 \Delta-(x-z)-(y-z) \\
\Leftrightarrow & y-x<2 \Delta-x+z-y+z \\
\Leftrightarrow & 2 y<2 \Delta+2 z \\
\Leftrightarrow & y-z<\Delta \\
\Leftrightarrow & |y-z|<\Delta \\
\Leftrightarrow & d(y, z)<\Delta
\end{aligned}
$$

Proposição 1.5.5. Nova métrica em $I_{[a, \Delta]}$. Seja a seguinte função:

$$
\begin{aligned}
g_{I}: I_{[a, \Delta]} \times I_{[a, \Delta]} & \longrightarrow \mathbb{R} \\
(x, y) & \longmapsto z=\min \{d(x, y), \Delta-d(x, y)\}
\end{aligned}
$$

Então, a função $g_{I}$ acima definida é uma métrica em $I_{[a, \Delta]}$.

Demonstração. Deve-se mostrar que a função $g_{I}$ obedece às propriedades que definem uma métrica: 
M1) Positividade: $\forall x, y \in I_{[a, \Delta]}, g_{I}(x, y) \geqslant 0$ e $g_{I}(x, x)=0$

$$
g_{I}(x, y)=\min \{d(x, y), \Delta-d(x, y)\}
$$

mas

$$
\begin{aligned}
& 0 \leqslant d(x, y)<\Delta \\
\Leftrightarrow & -\Delta \leqslant d(x, y)-\Delta<0 \\
\Leftrightarrow & 0<\Delta-d(x, y) \leqslant \Delta
\end{aligned}
$$

Portanto, pelas desigualdades 1.5 .1 e 1.5 .2 , tem se que $0 \leqslant g_{I}(x, y)<\Delta$, o que mostra a primeira parte da afirmação. A segunda parte é trivial, pois $d(x, x)=0$ e $g_{I}(x, x)=\min \{d(x, x), \Delta-d(x, x)\}=\min \{0, \Delta-0\}=0$.

M2) Positividade Estrita: $\forall x, y \in I_{[a, \Delta]}, g_{I}(x, y)=0 \Rightarrow x=y$

$$
\begin{aligned}
& g_{I}(x, y)=0 \\
\Rightarrow & \min \{d(x, y), \Delta-d(x, y)\}=0 \\
\Rightarrow & d(x, y)=0 \text { ou } \Delta-d(x, y)=0
\end{aligned}
$$

Como $d(x, y)<\Delta$, a segunda equação não tem solução, restando apenas a primeira. Como $d(x, y)$ é uma métrica, $d(x, y)=0 \Rightarrow x=y$.

M3) Simetria: $\forall x, y \in I_{[a, \Delta]}, g_{I}(x, y)=g_{I}(y, x)$

Usando a simetria de $d(x, y)$ :

$$
g_{I}(x, y)=\min \{d(x, y), \Delta-d(x, y)\}=\min \{d(y, x), \Delta-d(y, x)\}=g_{I}(y, x)
$$

M4) Desigualdade Triangular: $\forall x, y \in I_{[a, \Delta]}, g_{I}(x, y) \leqslant g_{I}(x, z)+g_{I}(z, y)$ 
Para provar a Desigualdade Triangular, serão necessárias quatro desigualdades:

a) $\quad d(x, y) \leqslant d(x, z)+d(z, y)$ ( Desigualdade Triangular de $\mathrm{d}(\cdot, \cdot))$

Como conseqüência da desigualdade triangular ([32]-3.2.1):

$$
\begin{aligned}
& d(x, y) \geqslant|d(x, z)-d(z, y)| \Rightarrow \\
& \Rightarrow\left\{\begin{array}{l}
d(x, y) \geqslant d(x, z)-d(z, y) \\
\mathrm{e} \\
d(x, y) \geqslant d(z, y)-d(x, z)
\end{array}\right.
\end{aligned}
$$

b) $\quad \Rightarrow\left\{\begin{array}{l}\Delta-d(x, y) \leqslant \Delta-d(x, z)+d(z, y) \\ \mathrm{e} \\ \Delta-d(x, y) \leqslant \Delta-d(z, y)+d(x, z)\end{array}\right.$

d) $\quad d(x, y)<\Delta-d(x, z)+\Delta-d(z, y)($ Proposição 1.5.4 )

Com estas quatro desigualdades, é possível escrever:

$$
\begin{aligned}
& g_{I}(x, y)=\min \{d(x, y), \Delta-d(x, y)\} \\
\leqslant & \min \{d(x, z)+d(z, y), \Delta-d(x, z)+d(z, y), \\
& \Delta-d(z, y)+d(x, z), \Delta-d(x, z)+\Delta-d(z, y)\} \\
= & \min \{d(x, z), \Delta-d(x, z)\}+\min \{d(z, y), \Delta-d(z, y)\} \\
= & g_{I}(x, z)+g_{I}(z, y)
\end{aligned}
$$

Definição 1.5.6. A topologia induzida pela métrica $g_{I}$ em $I_{[a, \Delta]}$ será denotada por $\mathcal{T}_{g_{I}}$. 
Proposição 1.5.7. Métrica em $\mathbb{G}_{\Delta}$. Seja a seguinte função:

$$
\begin{aligned}
g: \mathbb{G}_{\Delta} \times \mathbb{G}_{\Delta} & \longrightarrow \mathbb{R} \\
(X, Y) & \longmapsto z=g_{I}\left(\Pi_{[a, \Delta]}(X), \Pi_{[a, \Delta]}(Y)\right) \\
& \Rightarrow z=\min \left\{d\left(\Pi_{[a, \Delta]}(X), \Pi_{[a, \Delta]}(Y)\right), \Delta-d\left(\Pi_{[a, \Delta]}(X), \Pi_{[a, \Delta]}(Y)\right)\right\}
\end{aligned}
$$

Então, a função g acima definida é uma métrica em $\mathbb{G}_{\Delta}$.

Demonstração. Pela proposição 1.5.5, a função $g_{I}$ é uma métrica em $I_{[a, \Delta]}$. Portanto, como existe um mapeamento inversível entre $\mathbb{G}_{\Delta}$ e $I_{[a, \Delta]}$, se a afirmação $\forall x, y \in$ $I_{[a, \Delta]}, P\left(g_{I}(\cdot, \cdot), x, y\right)$ é verdadeira, então a seguinte seqüência de afirmações também é verdadeira:

$$
\begin{aligned}
& \forall x, y \in I_{[a, \Delta]}, P\left(g_{I}(\cdot, \cdot), x, y\right) \\
\Leftrightarrow & \forall \Pi_{[a, \Delta]}([x]), \Pi_{[a, \Delta]}([y]) \in \Pi_{[a, \Delta]}\left(\left[I_{[a, \Delta]}\right]\right), \\
& P\left(g_{I}\left(\Pi_{[a, \Delta]}([\cdot]), \Pi_{[a, \Delta]}([\cdot])\right), \Pi_{[a, \Delta]}([x]), \Pi_{[a, \Delta]}([y])\right) \\
\Leftrightarrow & \forall[x],[y] \in\left[I_{[a, \Delta]}\right], P\left(g_{I}([\cdot],[\cdot]),[x],[y]\right) \\
\Leftrightarrow & \forall X, Y \in \mathbb{G}_{\Delta}, P(g(\cdot, \cdot), X, Y)
\end{aligned}
$$

Portanto, se as quatro propriedades da métrica são verdadeiras na forma $\forall x, y \in$ $I_{[a, \Delta]}, P\left(g_{I}(\cdot, \cdot), x, y\right)$, então as mesmas quatro propriedades também são verdadeiras na forma $\forall X, Y \in \mathbb{G}_{\Delta}, P(g(\cdot, \cdot), X, Y)$, deste modo, se $g_{I}$ é uma métrica sobre o conjunto $I_{[a, \Delta]}$, então $g$ é uma métrica sobre $\mathbb{G}_{\Delta}$.

Definição 1.5.8. A topologia induzida pela métrica $g$ em $\mathbb{G}_{\Delta}$ será denotada por $\mathcal{T}_{\mathbb{G}_{\Delta}}$.

Lema 1.5.9. A métrica $g_{I}$ definida na proposição 1.5 .5 é invariante em relação à translações, isto é:

$$
\forall x, y, z \in I_{[a, \Delta]}, g_{I}(x, y)=g_{I}(x \oplus z, y \oplus z)
$$


Demonstração.

$$
\begin{aligned}
& d(x \oplus z, y \oplus z) \\
= & d(\bmod 2(x+z, \Delta, a), \bmod 2(y+z, \Delta, a)) \\
= & |\bmod 2(x+z, \Delta, a)-\bmod 2(y+z, \Delta, a)| \\
= & \left|\left(x+z-\left|\frac{x+z-a}{\Delta}\right| \Delta\right)-\left(y+z-\left|\frac{y+z-a}{\Delta}\right| \Delta\right)\right| \\
= & \left\{\begin{array}{l}
x+z-a<\Delta \text { e } y+z-a<\Delta \Rightarrow|x-y| \\
x+z-a<\Delta \text { e } y+z-a \geqslant \Delta \Rightarrow|x-y+\Delta|=\Delta-|y-x| \\
x+z-a \geqslant \Delta \text { e } y+z-a<\Delta \Rightarrow|x-y-\Delta|=\Delta-|x-y| \\
x+z-a \geqslant \Delta \text { e } y+z-a \geqslant \Delta \Rightarrow|x-\Delta-y+\Delta|=|x-y|
\end{array}\right.
\end{aligned}
$$

Então,

$$
\begin{aligned}
& g_{I}(x \oplus z, y \oplus z) \\
= & \min \{d(x \oplus z, y \oplus z), \Delta-d(x \oplus z, y \oplus z)\} \\
= & \min \{|x-y|, \Delta-|x-y|\} \\
= & \min \{d(x, y), \Delta-d(x, y)\} \\
= & g_{I}(x, y)
\end{aligned}
$$

Lema 1.5.10. A métrica $g$ definida na proposição 1.5 .7 é invariante em relação à translações, isto é:

$$
\forall X, Y, Z \in \mathbb{G}_{\Delta}, g(X, Y)=g(X \oplus Z, Y \oplus Z)
$$

Demonstração. É uma conseqüência trivial do lema 1.5.9 
Lema 1.5.11. O mapa $\Pi_{[a, \Delta]}:\left(\mathbb{G}_{\Delta}, g\right) \longrightarrow\left(I_{[a, \Delta]}, g_{I}\right)$ e sua inversa são contínuos, isto é, o mapa acima definido é na verdade um homeomorfismo.

Demonstração. É preciso mostrar que:

$$
\begin{aligned}
& \forall \epsilon>0 \in \mathbb{R}, \exists \delta>0 \in \mathbb{R} \mid \forall X_{0} \in \mathbb{G}_{\Delta}, g_{I}\left(\Pi_{[a, \Delta]}(X), \Pi_{[a, \Delta]}\left(X_{0}\right)\right)<\epsilon \\
& \Rightarrow g\left(X, X_{0}\right)<\delta
\end{aligned}
$$

$\operatorname{Mas} g_{I}\left(\Pi_{[a, \Delta]}(X), \Pi_{[a, \Delta]}\left(X_{0}\right)\right)=g\left(X, X_{0}\right)$. Tome portanto $\delta=\epsilon$ e a continuidade fica demonstrada. De modo inteiramente análogo mostra-se que $\Pi_{[a, \Delta]}^{-1}$ também é contínuo.

O lema 1.5.11 implica ([32]-3.10.2) que a continuidade de uma função no espaço $\left(\mathbb{G}_{\Delta}, g\right)$ implica na continuidade desta função composta com o homeomorfismo no espaço $\left(I_{[a, \Delta]}, g_{I}\right)$, e vice-versa composta com o homeomorfismo inverso. Ao mesmo tempo, se uma seqüência $\left\{x_{n}\right\}$ converge em $\left(\mathbb{G}_{\Delta}, g\right)$, então a seqüência $\left\{\Pi_{[a, \Delta]}\left(x_{n}\right)\right\}$ converge em $\left(I_{[a, \Delta]}, g_{I}\right)$, e vice-versa com o homeomorfismo inverso.

Lema 1.5.12. As seguintes relações são válidas entre as topologias acima definidas:
i) $\mathcal{T}_{g_{I}} \subset \mathcal{T}_{I_{[a, \Delta]}}$
ii) $\mathcal{T}_{g_{I}} \neq \mathcal{T}_{I_{[a, \Delta]}}$.

Demonstração.

i) para mostrar que $\mathcal{T}_{g_{I}} \subset \mathcal{T}_{I_{[a, \Delta]}}$ basta mostrar que toda bola aberta na métrica que gera a topologia $\mathcal{T}_{g_{I}}$ em torno de um ponto de $I_{[a, \Delta]}$ é também um conjunto aberto na topologia $\mathcal{T}_{I_{[a, \Delta]}}$, isto é, tem-se que mostrar que

$$
B=\left\{x \in I_{[a, \Delta]} \mid g_{I}(x, b)<\epsilon\right\} \in \mathcal{T}_{I_{[a, \Delta]}}
$$


Suponha em primeiro lugar que $b \leqslant a+\frac{\Delta}{2}$. Então existem dois casos:

1) $0<\epsilon \leqslant b-a$. Neste caso $B=(b-\epsilon, b+\epsilon)$, e este conjunto coincide com o conjunto $B_{\epsilon}(b)=\left\{x \in I_{[a, \Delta]} \mid d(x, b)<\epsilon\right\}$ das bolas abertas de raio $\epsilon$ em torno de $b$, e portanto $B$ é um conjunto aberto.

2) $b-a<\epsilon \leqslant \Delta$. Neste caso $B=[a, b+\epsilon) \cup(a+\Delta-\epsilon+b, a+\Delta)=B_{b+\epsilon}(a) \cup B_{\frac{\epsilon-b}{2}}\left(a+\Delta-\frac{\epsilon-b}{2}\right)$, que é a união de duas bolas abertas, e portanto também é um conjunto aberto.

O caso em que $b>a+\frac{\Delta}{2}$ pode ser desenvolvido de forma totalmente análoga.

ii) A fim de mostrar que $\mathcal{T}_{g_{I}} \neq \mathcal{T}_{I_{[a, \Delta]}}$, basta exibir um conjunto $A$ tal que $A \in$ $\mathcal{T}_{I_{[a, \Delta]}}$ e $A$ não pertence à $\mathcal{T}_{g_{I}}$. Seja então $A=\left\{x \in I_{[a, \Delta]}|| x-a \mid<\epsilon\right\}=$ $[a, \epsilon)$. Em termos da métrica que gera $\mathcal{T}_{[a, \Delta]}$, esse conjunto pode ser escrito como $A=\left\{x \in I_{[a, \Delta]} \mid d(x, a)<\epsilon\right\}$, que é uma bola aberta em torno do ponto $a$, e portanto é um conjunto aberto, isto é, $A \in \mathcal{T}_{I_{[a, \Delta]}}$. Porém, o ponto a pertence $A$, e não existe nenhuma bola aberta na métrica que gera a topologia $\mathcal{T}_{g_{I}}$ que esteja inteiramente contida em $\mathrm{A}$, isto é, não existe $\delta$ tal que o conjunto $B_{\delta}=\left\{x \in I_{[a, \Delta]} \mid g_{I}(x, a)<\delta\right\}=[a, \delta) \cup(a+\Delta-\delta, a+\Delta) \subset A$. Portanto, na topologia $\mathcal{T}_{g_{I}}, A$ não é aberto, pois não contém uma vizinhança de um de seus pontos.

Lema 1.5.13. Seja $V=(B, T)$ um par conjunto/topologia, isto é, um espaço topológico. As seguintes afirmativas são verdadeiras: 
i) Se a função $f:\left(I_{[a, \Delta]}, \mathcal{T}_{g_{I}}\right) \longrightarrow V$ é contínua, então tem-se que a função $f:\left(I_{[a, \Delta]}, \mathcal{T}_{I_{[a, \Delta]}}\right) \longrightarrow V$ também é contínua.

ii) Se a seqüência $\left\{x_{n}\right\}: \mathbb{N} \longrightarrow\left(I_{[a, \Delta]}, \mathcal{T}_{I_{[a, \Delta]}}\right)$ é convergente, então a seqüência $\left\{x_{n}\right\}: \mathbb{N} \longrightarrow\left(I_{[a, \Delta]}, \mathcal{T}_{g_{I}}\right)$ também é convergente.

Demonstração. É conseqüência direta do lema 1.5.12. Veja, por exemplo, [32]-3.9.

Em outras palavras, o Lema 1.5.13 diz que se uma função com domínio $I_{[a, \Delta]}$ é contínua na topologia $\mathcal{T}_{g_{I}}$, então a mesma função também é contínua em relação à topologia $\mathcal{T}_{I_{[a, \Delta]}}$, porém a recíproca não é necessariamente verdadeira. Além disso, toda seqüência convergente em $I_{[a, \Delta]}$ com a topologia $\mathcal{T}_{[a, \Delta]}$ também é convergente em $I_{[a, \Delta]}$ com a topologia $\mathcal{T}_{g_{I}}$, mas existem seqüências convergentes em $I_{[a, \Delta]}$ com a topologia $\mathcal{T}_{g_{I}}$ que não são convergentes em $I_{[a, \Delta]}$ com a topologia $\mathcal{T}_{[a, \Delta]}$. A fim de ilustrar os conceitos acima discutidos, tome por exemplo a seqüência $\left\{x_{n}\right\}=$ $\left\{a+\Delta-\frac{1}{n \Delta}, n \in \mathbb{N}\right\}$. Tal seqüência não converge em $\left(I_{[a, \Delta]}, \mathcal{T}_{I_{[a, \Delta]}}\right)$, pois o candidato a limite desta seqüência é o ponto $a+\Delta$, que não pertence à $I_{[a, \Delta]}$. No entanto, a mesma seqüência converge para o ponto $a$ em $\left(I_{[a, \Delta]}, \mathcal{T}_{g_{I}}\right)$. No que diz respeito à continuidade de funções, a condição para que uma função contínua com domínio em $\left(I_{[a, \Delta]}, \mathcal{T}_{I_{[a, \Delta]}}\right)$ também seja contínua com domínio em $\left(I_{[a, \Delta]}, \mathcal{T}_{g_{I}}\right)$ é que $\lim _{x \rightarrow a+\Delta} f(x)=$ $f(a)$.

\subsection{O Espaço Linear Topológico $\left(\mathbb{G}_{\Delta}, \oplus, \odot, g\right)$}

Será conveniente utilizar o espaço métrico $\left(\mathbb{G}_{\Delta}, g\right)$ com a estrutura algébrica de grupo de $\left(\mathbb{G}_{\Delta}, \oplus\right)$ e com um corpo sobre os números reais, de tal forma a permitir que sejam efetuadas multiplicações por números reais $(\odot)$ ). A norma a ser adotada no 
espaço linear $\left(\mathbb{G}_{\Delta}, \oplus, \odot\right)$ deveria ser derivada da métrica $g$ pois, conforme será visto mais adiante, esta métrica é tal que as operações " $\oplus$ " e "๑" são contínuas. Mas como também será visto posteriormente, a tentativa de criar esta norma neste espaço linear fracassou.

Proposição 1.6.1. Espaço Linear $\left(\mathbb{G}_{\Delta}, \oplus, \odot\right)$. O conjunto $\mathbb{G}_{\Delta}$ junto com os mapeamentos

$$
\begin{aligned}
\oplus: \mathbb{G}_{\Delta} \times \mathbb{G}_{\Delta} & \longrightarrow \mathbb{G}_{\Delta} \\
(X, Y) & \longmapsto Z=X \oplus Y=\left[\bmod 2\left(\Pi_{[a, \Delta]}(X)+\Pi_{[a, \Delta]}(Y), \Delta, a\right)\right] \\
e & \\
\odot: \mathbb{R} \times \mathbb{G}_{\Delta} & \longrightarrow \mathbb{G}_{\Delta} \\
(k, X) & \longmapsto Z=\left[\bmod 2\left(k \cdot \Pi_{[a, \Delta]}(X), \Delta, a\right)\right]
\end{aligned}
$$

formam um espaço linear.

Demonstração. Para mostrar que $\left(\mathbb{G}_{\Delta}, \oplus, \odot\right)$ é um espaço linear, deve-se mostrar que $\left(\mathbb{G}_{\Delta}, \oplus, \odot\right)$ satisfaz às seguintes nove condições:

EL1) $\forall X_{1}, X_{2} \in \mathbb{G}_{\Delta}, X_{1} \oplus X_{2}=X_{2} \oplus X_{1}$

Decorre trivialmente do lema 1.4.5-G4.

EL2) $\forall X_{1}, X_{2}, X_{3} \in \mathbb{G}_{\Delta}, X_{1} \oplus\left(X_{2} \oplus X_{3}\right)=\left(X_{1} \oplus X_{2}\right) \oplus X_{3}$

Decorre trivialmente do lema 1.4.5-G1.

EL3) $\exists ! 0 \in \mathbb{G}_{\Delta} \mid \forall X \in \mathbb{G}_{\Delta}, 0 \oplus X=X$

Decorre trivialmente do lema 1.4.5-G2. 
EL4) $\forall X \in \mathbb{G}_{\Delta}, \exists !(-X) \in \mathbb{G}_{\Delta} \mid X \oplus-X=0$

Decorre trivialmente do lema 1.4.5-G3.

EL5) $\forall \alpha, \beta \in \mathbb{R}, \forall X \in \mathbb{G}_{\Delta}, \alpha \odot(\beta \odot X)=(\alpha \beta) \odot X$

Seja $x=\Pi_{[a, \Delta]}(X)$. Então,

$$
\begin{aligned}
\alpha \odot(\beta \odot X) & =\alpha \odot\left[\bmod 2\left(\beta \cdot \Pi_{[a, \Delta]}(X), \Delta, a\right)\right] \\
& =\alpha \odot[\bmod 2(\beta \cdot x, \Delta, a)] \\
& =\alpha \odot[\beta \cdot x] \\
& =[\bmod 2(\alpha \cdot(\beta \cdot x), \Delta, a)] \\
& =[\bmod 2((\alpha \cdot \beta) \cdot x, \Delta, a)] \\
& =(\alpha \cdot \beta) \odot[x] \\
& =(\alpha \cdot \beta) \odot X
\end{aligned}
$$

EL6) $\forall X \in \mathbb{G}_{\Delta}, 1 \odot X=X$

Seja $x=\prod_{[a, \Delta]}(X)$. Então,

$$
\begin{aligned}
1 \odot X & =\left[\bmod 2\left(1 \cdot \Pi_{[a, \Delta]}(X), \Delta, a\right)\right] \\
& =[\bmod 2(1 \cdot x, \Delta, a)] \\
& =[\bmod 2(x, \Delta, a)] \\
& =[x] \\
& =X
\end{aligned}
$$

EL7) $\forall X \in \mathbb{G}_{\Delta}, 0 \odot X=0$

Seja $x=\Pi_{[a, \Delta]}(X)$. Então,

$$
0 \odot X=\left[\bmod 2\left(0 \cdot \Pi_{[a, \Delta]}(X), \Delta, a\right)\right]
$$




$$
\begin{aligned}
& =[\bmod 2(0 \cdot x, \Delta, a)] \\
& =[\bmod 2(0, \Delta, a)] \\
& =[0] \\
& =0
\end{aligned}
$$

EL8) $\forall X_{1}, X_{2} \in \mathbb{G}_{\Delta}, \forall \alpha \in \mathbb{R}, \alpha \odot\left(X_{1} \oplus X_{2}\right)=\alpha \odot X_{1} \oplus \alpha \odot X_{2}$ Sejam $x_{1}=\Pi_{[a, \Delta]}\left(X_{1}\right)$ e $x_{2}=\Pi_{[a, \Delta]}\left(X_{2}\right)$. Então,

$$
\begin{aligned}
\alpha \odot\left(X_{1} \oplus X_{2}\right) & =\alpha \odot\left[x_{1}+x_{2}\right] \\
& =\left[\bmod 2\left(\alpha \cdot\left(x_{1}+x_{2}\right), \Delta, a\right)\right] \\
& =\left[\bmod 2\left(\alpha \cdot x_{1}+\alpha \cdot x_{2}, \Delta, a\right)\right] \\
& =\left[\alpha \cdot x_{1}+\alpha \cdot x_{2}\right] \\
& =\left[\alpha \cdot x_{1}\right] \oplus\left[\alpha \cdot x_{2}\right] \\
& =\left[\bmod 2\left(\alpha \cdot x_{1}, \Delta, a\right)\right] \oplus\left[\bmod 2\left(\alpha \cdot x_{2}, \Delta, a\right)\right] \\
& =\alpha \odot\left[x_{1}\right] \oplus \alpha \odot\left[x_{2}\right] \\
& =\alpha \odot X_{1} \oplus \alpha \odot X_{2}
\end{aligned}
$$

EL9) $\forall X \in \mathbb{G}_{\Delta}, \forall \alpha, \beta \in \mathbb{R},(\alpha+\beta) \odot X=\alpha \odot X \oplus \beta \odot X$

Seja $x=\Pi_{[a, \Delta]}(X)$. Então,

$$
\begin{aligned}
(\alpha+\beta) \odot X & =\left[\bmod 2\left((\alpha+\beta) \cdot \Pi_{[a, \Delta]}(X), \Delta, a\right)\right] \\
& =[\bmod 2((\alpha+\beta) \cdot x, \Delta, a)] \\
& =[\bmod 2(\alpha \cdot x+\beta \cdot x, \Delta, a)] \\
& =[\alpha \cdot x+\beta \cdot x]
\end{aligned}
$$




$$
\begin{aligned}
& =[\alpha \cdot x] \oplus[\beta \cdot x] \\
& =\alpha \odot[x] \oplus \beta \odot[x] \\
& =\alpha \odot X \oplus \beta \odot X
\end{aligned}
$$

Proposição 1.6.2. O mapeamento

$$
\begin{aligned}
\|\cdot\|_{g}: \mathbb{G}_{\Delta} & \longrightarrow \mathbb{R} \\
X & \longmapsto y=g(X, 0),
\end{aligned}
$$

ao contrário do que seria desejável, não é uma norma em $\left(\mathbb{G}_{\Delta}, \oplus, \odot\right)$.

Demonstração. Para mostrar que $\|\cdot\|_{g}$ não é uma norma em $\left(\mathbb{G}_{\Delta}, \oplus, \odot\right)$, deve-se mostrar que $\|\cdot\|_{g}$ falha em satisfazer à pelo menos uma das seguintes quatro condições:

N1) $\forall X \in \mathbb{G}_{\Delta},\|X\|_{g} \geqslant 0$ (Positividade)

Decorre trivialmente da propriedade M1 da métrica:

$$
\begin{aligned}
\forall X, Y \in \mathbb{G}_{\Delta}, g(X, Y) \geqslant 0 & \Rightarrow \forall X \in \mathbb{G}_{\Delta}, g(X, 0) \geqslant 0 \\
& \Rightarrow \forall X \in \mathbb{G}_{\Delta},\|X\|_{g} \geqslant 0
\end{aligned}
$$

N2) $\forall X, Y \in \mathbb{G}_{\Delta},\|X \oplus Y\|_{g} \leqslant\|X\|_{g}+\|Y\|_{g}$ (Desigualdade Triangular)

Seja $x=\Pi_{[a, \Delta]}(X)$. Note que

$$
\begin{aligned}
\|X\|_{g} & =g(X, 0) \\
(\text { Lema 1.5.10) } & =g(X \oplus(-X), 0 \oplus(-X)) \\
& =g(0,-X)
\end{aligned}
$$




$$
\begin{aligned}
(\text { simetria de } g) & =g(-X, 0) \\
& =\|-X\|_{g}
\end{aligned}
$$

Então,

$$
\begin{aligned}
\|X \oplus Y\|_{g} & =g(X \oplus Y, 0) \\
(\text { Lema 1.5.10) } & =g(X \oplus Y \oplus(-Y), 0 \oplus(-Y)) \\
& =g(X,-Y) \\
\text { (Prop. 1.5.5-M4) } & \leqslant g(X, Z)+g(Z,-Y) \\
(Z=0, \text { e simetria de g) } & =g(X, 0)+g(-Y, 0) \\
& =\|X\|_{g}+\|-Y\|_{g} \\
& =\|X\|_{g}+\|Y\|_{g}
\end{aligned}
$$

N3) $\forall \alpha \in \mathbb{R}, \forall X \in \mathbb{G}_{\Delta},\|\alpha \odot X\|_{g}=|\alpha| \cdot\|X\|_{g}$ (Homogeneidade)

Infelizmente a função $\|\cdot\|_{g}$ não satisfaz a essa propriedade, pois claramente a igualdade é impossível quando $X$ é fixo e $\alpha$ cresce sem limite, uma vez que o lado esquerdo da equação é limitado por $\frac{\Delta}{2}$. Uma tentativa de "forçar" a homogeneidade seria mudar o significado da igualdade para uma igualdade módulo $\frac{\Delta}{2}$ da seguinte forma: $\forall \alpha \in \mathbb{R}, \forall X \in \mathbb{G}_{\Delta},\|\alpha \odot X\|_{g} \stackrel{(\bmod }{=}{ }^{\left.\frac{\Delta}{2}\right)}|\alpha|\|X\|_{g}$. Mas mesmo neste caso a tentativa fracassa, pois suponha que $\alpha X=\frac{\Delta}{2}+\epsilon$, onde $\epsilon$ é próximo de zero. Então $\|\alpha \odot X\|_{g}=\frac{\Delta}{2}-\epsilon$ e $|\alpha|\|X\|_{g} \stackrel{\left(\bmod \frac{\Delta}{2}\right)}{=} \epsilon$.

N4) $\|X\|_{g}=0 \Leftrightarrow X=0$ (Positiva Definida)

$$
\|X\|_{g}=0 \Leftrightarrow g(X, 0)=0 \Leftrightarrow X=0
$$


Como foi visto na demonstração, a função candidata a norma derivada da métrica em $\left(\mathbb{G}_{\Delta}, g\right)$ não satisfaz às condições necessárias pois falha na homogeneidade. Mas apesar disso a perda não foi muito grande porque como será visto mais adiante, a regra para caracterização da continuidade da uma transformação linear $L:\left(\mathbb{G}_{\Delta}, g\right) \longrightarrow$ $\left(\mathbb{G}_{\Delta}, g\right)$ é simples e não depende de uma norma. E enquanto o número $n$ de dimensões for mantido finito, é possível criar espaços lineares $\mathbb{G}_{\Delta}^{n}$ com métricas que preservem esta regra de continuidade das transformações lineares, sem que se tenha que colocar o problema da limitação.

Proposição 1.6.3. O espaço linear topológico $\left(\mathbb{G}_{\Delta}, \oplus, \odot, g\right)$ é completo.

Demonstração. $I_{[a, \Delta]} \cup\{a+\Delta\}$ é um subconjunto fechado de $(\mathbb{R}, d)$. Portanto, como $\mathbb{R}$ é completo, toda seqüência de Cauchy em $\left(I_{[a, \Delta]} \cup\{a+\Delta\}, d\right)$ converge. Deste modo, toda seqüência de Cauchy em $\left(I_{[a, \Delta]}, d\right)$ converge, exceto as que convergiam no espaço anterior para o ponto $a+\Delta$. Seja $\left\{x_{n}\right\}$ uma dessas seqüências. Então:

$$
\begin{gathered}
\lim _{n \rightarrow \infty} x_{n}=a+\Delta \\
\Leftrightarrow \quad\left(\lim _{n \rightarrow \infty} x_{n}\right)-a=\Delta
\end{gathered}
$$

Continuidade de "-" $+[32]-3.7 .2 \Leftrightarrow \lim _{n \rightarrow \infty}\left(x_{n}-a\right)=\Delta$

$$
\begin{aligned}
\left(x_{n} \geqslant a, \forall n\right) & \Rightarrow \lim _{n \rightarrow \infty}\left|x_{n}-a\right|=\Delta \\
& \Leftrightarrow \lim _{n \rightarrow \infty} d\left(x_{n}, a\right)=\Delta \\
\text { Prop. 1.5.1 } & \Leftrightarrow \Delta-\lim _{n \rightarrow \infty} d\left(x_{n}, a\right)=0
\end{aligned}
$$

Continuidade de "-" $+[32]-3.7 .2 \Leftrightarrow \lim _{n \rightarrow \infty}\left(\Delta-d\left(x_{n}, a\right)\right)=0$

$$
\Rightarrow \min \left\{\lim _{n \rightarrow \infty} d\left(x_{n}, a\right), \lim _{n \rightarrow \infty}\left(\Delta-d\left(x_{n}, a\right)\right)\right\}=0
$$

Continuidade de "min" $+[32]-3.7 .2 \Leftrightarrow \lim _{n \rightarrow \infty} \min \left\{d\left(x_{n}, a\right), \Delta-d\left(x_{n}, a\right)\right\}=0$ 


$$
\Leftrightarrow \lim _{n \rightarrow \infty} g_{I}\left(x_{n}, a\right)=0
$$

Portanto, na métrica $g_{I}$ estas seqüências convergem agora para o ponto $a$. E nesta mesma métrica, usando um desenvolvimento semelhante, as seqüências de Cauchy que convergiam para outros pontos continuam convergindo para os mesmos pontos. Então toda seqüência de Cauchy em $\left(I_{[a, \Delta]}, g_{I}\right)$ converge. Como $\left(\mathbb{G}_{\Delta}, g\right)$ e $\left(I_{[a, \Delta]}, g_{I}\right)$ são homeomorfos, toda seqüência de Cauchy em $\left(\mathbb{G}_{\Delta}, g\right)$ também converge, logo $\left(\mathbb{G}_{\Delta}, g\right)$ é completo.

As proposições a seguir demonstram as afirmações que foram feitas no início da seção anterior com relação à continuidade das operações que definem o espaço linear topológico $\left(\mathbb{G}_{\Delta}, \oplus, \odot, g\right)$.

Proposição 1.6.4. Seja $y \in I_{[a, \Delta]}$ um ponto dado e seja $f$ a seguinte função:

$$
\begin{aligned}
f: I_{[a, \Delta]} & \longrightarrow I_{[a, \Delta]} \\
x & \longmapsto z=x \oplus y
\end{aligned}
$$

Então $f:\left(I_{[a, \Delta]}, \mathcal{T}_{I_{[a, \Delta]}}\right) \longrightarrow\left(I_{[a, \Delta]}, \mathcal{T}_{I_{[a, \Delta]}}\right)$ é contínua em todos os pontos, exceto talvez no ponto $x_{c}=\bmod 2(a-y, \Delta, a)$, e será contínua em $x_{c}$ se e somente se $y=a$.

Demonstração. Se $\epsilon \geqslant \Delta$, então, pela proposição 1.5.3, qualquer valor de $\delta$ satisfaz $\left|d\left(x, x_{0}\right)\right|<\delta \Rightarrow\left|d\left(x \oplus y, x_{0} \oplus y\right)\right|<\Delta<\epsilon$. Se $\epsilon<\Delta$, então tem-se que analisar vários casos:

i) $x_{0}<x_{c}$ e $x<x_{c}$

$$
d\left(x \oplus y, x_{0} \oplus y\right)
$$




$$
\begin{aligned}
= & \left|\bmod 2(x+y, \Delta, a)-\bmod 2\left(x_{0}+y, \Delta, a\right)\right| \\
= & \left|\left(x+y-\left\lfloor\frac{x+y-a}{\Delta}\right\rfloor \Delta\right)-\left(x_{0}+y-\left\lfloor\frac{x_{0}+y-a}{\Delta}\right\rfloor \Delta\right)\right| \\
= & \left|x-x_{0}-(m-n) \Delta\right| \\
& \operatorname{Mas} x_{0}<x_{c} \text { e } x<x_{c} \Rightarrow m-n=0, \log \mathrm{O} \\
& d\left(x \oplus y, x_{0} \oplus y\right)=\left|x-x_{0}\right|
\end{aligned}
$$

Portanto,

$$
\begin{aligned}
& d\left(x, x_{0}\right)<\delta \\
\Leftrightarrow & \left|x-x_{0}\right|<\delta \\
\Leftrightarrow & d\left(x \oplus y, x_{0} \oplus y\right)<\delta \\
(\delta=\epsilon) \Leftrightarrow & d\left(x \oplus y, x_{0} \oplus y\right)<\epsilon
\end{aligned}
$$

ii) $x_{c}<x_{0}$ e $x_{c}<x$

$$
\begin{aligned}
& d\left(x \oplus y, x_{0} \oplus y\right) \\
= & \left|\bmod 2(x+y, \Delta, a)-\bmod 2\left(x_{0}+y, \Delta, a\right)\right| \\
= & \left|\left(x+y-\left\lfloor\frac{x+y-a}{\Delta}\right\rfloor \Delta\right)-\left(x_{0}+y-\left\lfloor\frac{x_{0}+y-a}{\Delta}\right\rfloor \Delta\right)\right| \\
= & \left|x-x_{0}-(m-n) \Delta\right| \\
& \operatorname{Mas} x_{c}<x_{0} \text { e } x_{c}<x \Rightarrow m-n=0, \log \mathrm{O} \\
& d\left(x \oplus y, x_{0} \oplus y\right)=\left|x-x_{0}\right|
\end{aligned}
$$

Portanto,

$$
\begin{aligned}
& d\left(x, x_{0}\right)<\delta \\
\Leftrightarrow & \left|x-x_{0}\right|<\delta
\end{aligned}
$$




$$
\begin{aligned}
& \Leftrightarrow d\left(x \oplus y, x_{0} \oplus y\right)<\delta \\
(\delta=\epsilon) & \Leftrightarrow d\left(x \oplus y, x_{0} \oplus y\right)<\epsilon
\end{aligned}
$$

iii) $x<x_{c}=x_{0}$

$$
\begin{aligned}
& d\left(x \oplus y, x_{0} \oplus y\right) \\
= & d\left(x \oplus y, x_{c} \oplus y\right) \\
= & d(x \oplus y, a) \\
= & |\bmod 2(x+y, \Delta, a)-a| \\
= & |\bmod 2(x+y, \Delta, a)-a| \\
= & \left|\left(x+y-\left\lfloor\frac{x+y-a}{\Delta}\right\rfloor \Delta\right)-a\right| \\
\left(x<x_{c} \Rightarrow\left\lfloor\frac{x+y-a}{\Delta}\right\rfloor=0\right)= & |x+y-a|
\end{aligned}
$$

Portanto, se $y \neq a$,

$$
\begin{aligned}
& d\left(x, x_{0}\right)<\delta \\
& \Leftrightarrow d\left(x, x_{c}\right)<\delta \\
& \Leftrightarrow\left|x-x_{c}\right|<\delta \\
& \left.\Leftrightarrow \mid x-\left(a-y-\mid \frac{a-y}{\Delta}\right\rfloor \Delta\right) \mid<\delta \\
\left.\left(y \in I_{[a, \Delta]} \Rightarrow \mid \frac{a-y}{\Delta}\right\rfloor=-1\right) & \Leftrightarrow|x+y-a-\Delta|<\delta \\
\left(x+y-a \in I_{[a, \Delta]}\right) & \Leftrightarrow \Delta-|x+y-a|<\delta \\
& \Leftrightarrow d\left(x \oplus y, x_{0} \oplus y\right)>\Delta-\delta
\end{aligned}
$$

Neste caso a função não é contínua no limite pela esquerda em $x_{0}=x_{c}$. Mas se $y=a$,

$$
\left(y=a \Rightarrow\left\lfloor\frac{a-y}{\Delta}\right\rfloor=0\right) \Leftrightarrow|x+y-a|<\delta
$$




$$
\begin{aligned}
& \Leftrightarrow d\left(x \oplus y, x_{0} \oplus y\right)<\delta \\
(\delta=\epsilon) & \Leftrightarrow d\left(x \oplus y, x_{0} \oplus y\right)<\epsilon
\end{aligned}
$$

Então se $y=a$ a função é contínua pela esquerda em $x_{0}=x_{c}$.

iv) $x_{c}=x_{0}<x$

$$
\begin{aligned}
& d\left(x \oplus y, x_{0} \oplus y\right) \\
= & d\left(x \oplus y, x_{c} \oplus y\right) \\
= & d(x \oplus y, a) \\
= & |\bmod 2(x+y, \Delta, a)-a| \\
= & |\bmod 2(x+y, \Delta, a)-a| \\
= & \left|\left(x+y-\left\lfloor\frac{x+y-a}{\Delta}\right\rfloor \Delta\right)-a\right| \\
\left(x>x_{c} \Rightarrow\left\lfloor\frac{x+y-a}{\Delta}\right\rfloor=1\right)= & |x+y-a-\Delta|
\end{aligned}
$$

Portanto,

$$
\begin{aligned}
& d\left(x, x_{0}\right)<\delta \\
& \Leftrightarrow d\left(x, x_{c}\right)<\delta \\
& \Leftrightarrow\left|x-x_{c}\right|<\delta \\
& \left.\Leftrightarrow \mid x-\left(a-y-\mid \frac{a-y}{\Delta}\right\rfloor \Delta\right) \mid<\delta \\
\left.\left(y \in I_{[a, \Delta]} \Rightarrow \mid \frac{a-y}{\Delta}\right\rfloor=-1\right) & \Leftrightarrow|x+y-a-\Delta|<\delta \\
& \Leftrightarrow d\left(x \oplus y, x_{0} \oplus y\right)<\delta \\
(\delta=\epsilon) & \Leftrightarrow d\left(x \oplus y, x_{0} \oplus y\right)<\epsilon
\end{aligned}
$$


Proposição 1.6.5. Sejam y e f como na proposição 1.6.4.

Então $f:\left(I_{[a, \Delta]}, \mathcal{T}_{g_{I}}\right) \longrightarrow\left(I_{[a, \Delta]}, \mathcal{T}_{g_{I}}\right)$ é contínua $\forall y \in I_{[a, \Delta]}$.

Demonstração. Pela proposição 1.5.9, a métrica $g_{I}$ é invariante em relação a translações, portanto:

$$
\begin{aligned}
& g_{I}\left(x, x_{0}\right)<\delta \\
\Leftrightarrow & g_{I}\left(x \oplus y, x_{0} \oplus y\right)<\delta \\
(\delta=\epsilon) \Leftrightarrow & g_{I}\left(x \oplus y, x_{0} \oplus y\right)<\epsilon
\end{aligned}
$$

A simplicidade da demonstração anterior chega a ser desconcertante. Como veremos a seguir, as coisas não funcionam tão bem para o produto por escalar.

Proposição 1.6.6. Defina a seguinte operação:

$$
\begin{aligned}
\odot: \mathbb{R} \times I_{[a, \Delta]} & \longrightarrow I_{[a, \Delta]} \\
(k, x) & \longmapsto Z=\bmod 2(k \cdot x, \Delta, a)
\end{aligned}
$$

Então, vale a seguinte propriedade que justifica o abuso de notação:

$$
k \odot[x]=[k \odot x]
$$

Demonstração. A demonstração faz uso direto das definições das duas operações e é bastante óbvia.

$$
\begin{aligned}
& k \odot[x] \\
= & {\left[\bmod 2\left(k \cdot \Pi_{[a, \Delta]}([x]), \Delta, a\right)\right] } \\
= & {[\bmod 2(k \cdot x, \Delta, a)] }
\end{aligned}
$$




$$
=[k \odot x]
$$

Proposição 1.6.7. Seja $k \in \mathbb{R}, a \in \mathbb{R}, \Delta \in(0,+\infty)$ e seja $f$ a seguinte função:

$$
\begin{aligned}
f:\left(I_{[a, \Delta]}, \mathcal{T}_{I_{[a, \Delta]}}\right) & \longrightarrow\left(I_{[a, \Delta]}, \mathcal{T}_{I_{[a, \Delta]}}\right) \\
x & \longmapsto y=k \odot x
\end{aligned}
$$

Seja $x_{i}$ a i-ésima raiz da equação $k \odot x=a$. Então as seguintes afirmações são verdadeiras:

i) Se $x \neq x_{i} \Rightarrow f$ é contínua em $x$.

ii) Se $x=x_{i}, k>0$ e $x_{i} \neq a, f$ é contínua pela direita e descontínua pela esquerda em $x$.

iii) Se $x=x_{i}, k>0$ e $x_{i}=a, f$ é contínua pela direita e consequentemente contínua em $x$, pois não há esquerda neste caso.

iv) Se $x=x_{i}, k<0$ e $x_{i} \neq a$, $f$ é contínua pela esquerda e descontínua pela direita em $x$.

v) Se $x=x_{i}, k<0$ e $x_{i}=a, f$ é descontínua pela direita e consequentemente descontínua em $x$, pois não há esquerda neste caso.

Demonstração. Em primeiro lugar, analise as raízes da equação $k \odot x=a$ :

$$
\begin{aligned}
& k \odot x=a \\
\Leftrightarrow & \bmod 2(k x, \Delta, a)=a \\
\Leftrightarrow & k x-\left\lfloor\frac{k x-a}{\Delta}\right\rfloor \Delta=a
\end{aligned}
$$




$$
\left(l=\left\lfloor\frac{k x-a}{\Delta}\right\rfloor\right) \Leftrightarrow x=\frac{a+l \Delta}{k} .
$$

Mas $x \in I_{[a, \Delta]}$, de modo que

$$
\begin{aligned}
& a \leqslant x<a+\Delta \\
\Leftrightarrow & a \leqslant \frac{a+l \Delta}{k}<a+\Delta \\
\Leftrightarrow & \left\{\begin{array}{l}
k<0 \Rightarrow k a+k \Delta<a+l \Delta \leqslant k a \\
k>0 \Rightarrow k a \leqslant a+l \Delta<k a+k \Delta
\end{array}\right. \\
\Leftrightarrow & \left\{\begin{array}{l}
k<0 \Rightarrow \frac{(k-1) a}{\Delta}+k<l \leqslant \frac{(k-1) a}{\Delta} \\
k>0 \Rightarrow \frac{(k-1) a}{\Delta} \leqslant l<\frac{(k-1) a}{\Delta}+k
\end{array}\right.
\end{aligned}
$$

Portanto, existem tantas raízes quantos forem os valores de $l \in \mathbb{Z}$ distintos satisfazendo à relação 1.6.2. Seja então $n$ o número de valores de $l$ que satisfazem à relação 1.6.2. Fazendo $l=m+i$ pode-se escrever

$$
x_{i}=\frac{a+(m+i) \Delta}{k}, i=1, \ldots, n . \Rightarrow m+i=\frac{k x_{i}-a}{\Delta}
$$

Existem a princípio $n+1$ intervalos de valores possíveis para $x$, delimitados pelas raízes da equação $k \odot x=a$, podendo haver intervalos que sejam vazios ou singletos, no caso de $x_{i}=a$ :

$$
\left\{\begin{array}{l}
k<0 \Rightarrow\left\{\begin{array}{l}
L_{0}=\left\{x \in \mathbb{R}: x_{1}<x<a+\Delta\right\} \\
L_{i}=\left\{x \in \mathbb{R}: x_{i+1}<x<x_{i}, i=1, \ldots, n-1\right\} \\
L_{n}=\left\{x \in \mathbb{R}: a \leqslant x<x_{n}\right\} \\
L_{0}=\left\{x \in \mathbb{R}: a \leqslant x<x_{1}\right\} \\
L_{i}=\left\{x \in \mathbb{R}: x_{i}<x<x_{i+1}, i=1, \ldots, n-1\right\} \\
L_{n}=\left\{x \in \mathbb{R}: x_{n}<x<a+\Delta\right\}
\end{array}\right.
\end{array}\right.
$$


Então,

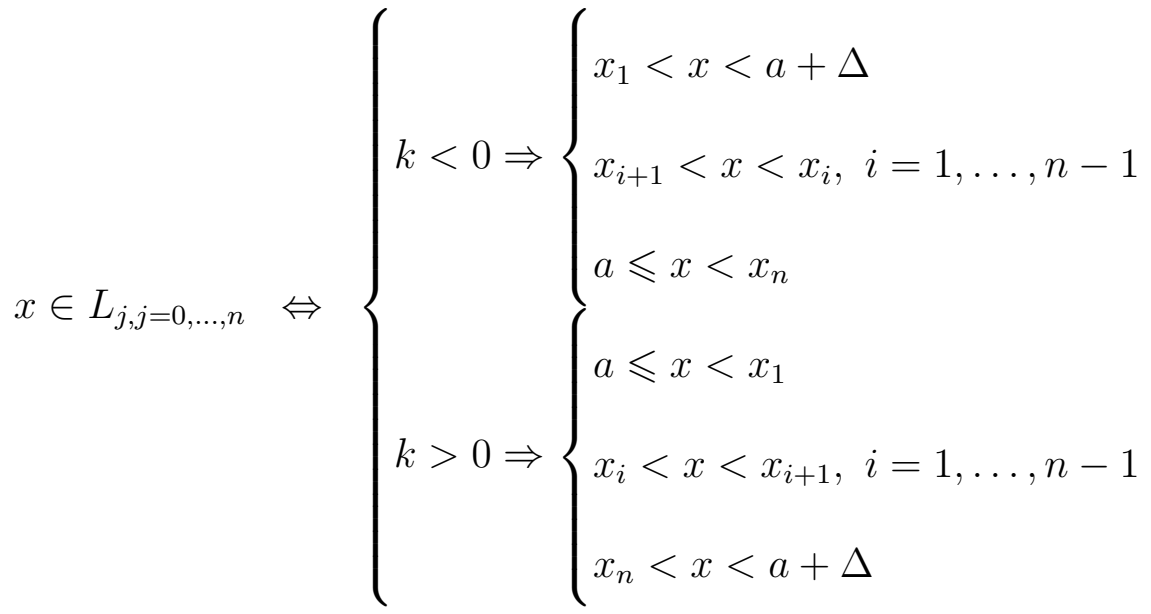

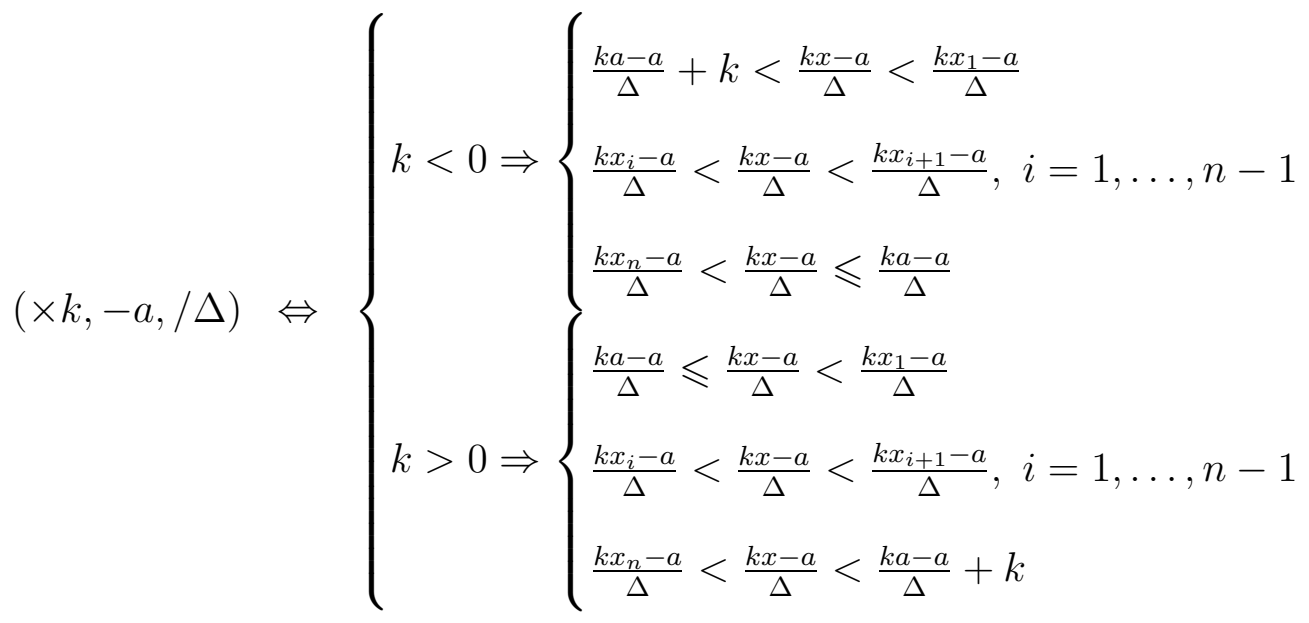

$$
\begin{aligned}
& \left(\text { Eq.1.6.3) } \Leftrightarrow \left\{\begin{array}{l}
k<0 \Rightarrow\left\{\begin{array}{l}
\frac{(k-1) a}{\Delta}+k<\frac{k x-a}{\Delta}<m+1 \\
m+i<\frac{k x-a}{\Delta}<m+i+1, i=1, \ldots, n-1 \\
m+n<\frac{k x-a}{\Delta} \leqslant \frac{(k-1) a}{\Delta}
\end{array}\right. \\
k>0 \Rightarrow\left\{\begin{array}{l}
\frac{(k-1) a}{\Delta} \leqslant \frac{k x-a}{\Delta}<m+1 \\
m+i<\frac{k x-a}{\Delta}<m+i+1, i=1, \ldots, n-1 \\
m+n<\frac{k x-a}{\Delta}<\frac{(k-1) a}{\Delta}+k
\end{array}\right.
\end{array}\right.\right.
\end{aligned}
$$


As relações anteriores implicam em

$$
\left\lfloor\frac{k x-a}{\Delta} \mid=l_{j}=\left\{\begin{array}{l}
k<0 \Rightarrow\left\{\begin{array}{l}
x \in L_{0} \Rightarrow m \\
x \in L_{i} \Rightarrow m+i \\
x \in L_{n} \Rightarrow m+n
\end{array}\right. \\
k>0 \Rightarrow\left\{\begin{array}{l}
x \in L_{0} \Rightarrow m \\
x \in L_{i} \Rightarrow m+i \\
x \in L_{n} \Rightarrow m+n
\end{array}\right.
\end{array}\right.\right.
$$

Sejam então $x$ e $x_{0} \in L_{j}, j=0, \ldots, n$, e $x_{0} \neq a$.

$$
\begin{aligned}
d\left(k \odot x, k \odot x_{0}\right) & =\left|\bmod 2(k x, \Delta, a)-\bmod 2\left(k x_{0}, \Delta, a\right)\right| \\
& =\left|\left(k x-\left\lfloor\frac{k x-a}{\Delta}\right\rfloor \Delta\right)-\left(k x_{0}-\left\lfloor\frac{k x_{0}-a}{\Delta}\right\rfloor \Delta\right)\right| \\
& =\left|k\left(x-x_{0}\right)-\left(\left\lfloor\frac{k x-a}{\Delta}\right\rfloor-\left\lfloor\frac{k x_{0}-a}{\Delta}\right\rfloor\right) \Delta\right| \\
& =\left|k\left(x-x_{0}\right)-\left(l_{j}-l_{j}\right) \Delta\right| \\
& =\left|k\left(x-x_{0}\right)\right| \\
& =|k| \cdot\left|x-x_{0}\right| \\
& =|k| \cdot d\left(x, x_{0}\right)
\end{aligned}
$$

Assim,

$$
\begin{aligned}
& d\left(x, x_{0}\right)<\delta \\
\Leftrightarrow & \frac{d\left(k \odot x, k \odot x_{0}\right)}{|k|}<\delta \\
(\delta=|k| \cdot \epsilon) \Leftrightarrow & d\left(k \odot x, k \odot x_{0}\right)<\epsilon
\end{aligned}
$$

Provando a afirmação i). Suponha agora que $k>0, x_{0}=x_{i} \neq a$ e $x>x_{0}$ ( limite 
pela direita ):

$$
\begin{aligned}
d\left(k \odot x, k \odot x_{0}\right) & =d\left(k \odot x, k \odot x_{i}\right) \\
& =d(k \odot x, a) \\
& =|\bmod 2(k x, \Delta, a)-a| \\
& =\left|\left(k x-\left|\frac{k x-a}{\Delta}\right| \Delta\right)-a\right| \\
& =\left|k x-l_{j} \Delta-a\right| \\
& =|k| \cdot\left|x-\frac{a+l_{j} \Delta}{k}\right| \\
(\text { Eq. 1.6.3) } & =|k| \cdot\left|x-x_{i}\right| \\
& =|k| \cdot d\left(x, x_{i}\right) \\
& =|k| \cdot d\left(x, x_{0}\right)
\end{aligned}
$$

Assim,

$$
\begin{aligned}
& d\left(x, x_{0}\right)<\delta \\
\Leftrightarrow & \frac{d\left(k \odot x, k \odot x_{0}\right)}{|k|}<\delta \\
(\delta=|k| \cdot \epsilon) \Leftrightarrow & d\left(k \odot x, k \odot x_{0}\right)<\epsilon,
\end{aligned}
$$

mostrando que a função é contínua pela direita em $x_{0}=x_{i}$. Assuma agora as mesmas hipóteses anteriores, exceto que $x<x_{0}$ ( limite pela esquerda ). Então,

$$
\begin{aligned}
d\left(k \odot x, k \odot x_{0}\right) & =d\left(k \odot x, k \odot x_{i}\right) \\
& =d(k \odot x, a) \\
& =|\bmod 2(k x, \Delta, a)-a| \\
& \left.=\mid\left(k x-\mid \frac{k x-a}{\Delta}\right\rfloor \Delta\right)-a \mid \\
& =\left|k x-l_{j-1} \Delta-a\right|
\end{aligned}
$$




$$
\begin{aligned}
& =|k| \cdot\left|x-\frac{a+l_{j-1} \Delta}{k}\right| \\
\left(l_{j-1}=l_{j}-1\right) & =|k| \cdot\left|x-x_{i}+\frac{\Delta}{k}\right| \\
& =|k| \cdot\left(\frac{\Delta}{|k|}-\left|x-x_{i}\right|\right) \\
& =\Delta-|k| \cdot d\left(x, x_{i}\right) \\
& =\Delta-|k| \cdot d\left(x, x_{0}\right)
\end{aligned}
$$

Assim,

$$
\begin{aligned}
& d\left(x, x_{0}\right)<\delta \\
\Leftrightarrow & \frac{\Delta-d\left(k \odot x, k \odot x_{0}\right)}{|k|}<\delta \\
(\delta=|k| \cdot \epsilon) \Leftrightarrow & d\left(k \odot x, k \odot x_{0}\right)>\Delta-\epsilon,
\end{aligned}
$$

mostrando que a função é descontínua pela esquerda em $x_{0}=x_{i}$, o que completa a demonstração da afirmação ii). No caso da afirmação iii), as hipóteses são $k>0$, $x_{0}=x_{i}=a, x>x_{0}$ ( limite pela direita $), \operatorname{logo}$

$$
\begin{aligned}
d\left(k \odot x, k \odot x_{0}\right) & =d(k \odot x, k \odot a) \\
& =d(k \odot x, a) \\
& =|\bmod 2(k x, \Delta, a)-a| \\
& =\left|\left(k x-\left|\frac{k x-a}{\Delta}\right| \Delta\right)-a\right| \\
& =\left|k x-l_{1} \Delta-a\right| \\
& =|k| \cdot\left|x-\frac{a+l_{1} \Delta}{k}\right| \\
& =|k| \cdot\left|x-x_{1}\right| \\
& =|k| \cdot d(x, a) \\
& =|k| \cdot d\left(x, x_{0}\right)
\end{aligned}
$$


Assim,

$$
\begin{aligned}
& d\left(x, x_{0}\right)<\delta \\
\Leftrightarrow & \frac{d\left(k \odot x, k \odot x_{0}\right)}{|k|}<\delta \\
(\delta=|k| \cdot \epsilon) \Leftrightarrow & d\left(k \odot x, k \odot x_{0}\right)<\epsilon,
\end{aligned}
$$

mostrando que neste caso a função é contínua pela direita, o que prova a afirmação iii). Suponha agora que $k<0, x_{0}=x_{i} \neq a$ e $x>x_{0}$ ( limite pela direita ):

$$
\begin{aligned}
d\left(k \odot x, k \odot x_{0}\right) & =d(k \odot x, a) \\
& =|\bmod 2(k x, \Delta, a)-a| \\
& =\left|\left(k x-\left|\frac{k x-a}{\Delta}\right| \Delta\right)-a\right| \\
& =\left|k x-l_{j-1} \Delta-a\right| \\
& =|k| \cdot\left|x-\frac{a+l_{j-1} \Delta}{k}\right| \\
\left(l_{j-1}=l_{j}-1\right) & =|k| \cdot\left|x-x_{i}+\frac{\Delta}{k}\right| \\
& =|k| \cdot\left(\frac{\Delta}{|k|}-\left|x-x_{i}\right|\right) \\
& =\Delta-|k| \cdot d\left(x, x_{i}\right) \\
& =\Delta-|k| \cdot d\left(x, x_{0}\right)
\end{aligned}
$$

Assim,

$$
\begin{aligned}
& d\left(x, x_{0}\right)<\delta \\
\Leftrightarrow & \frac{\Delta-d\left(k \odot x, k \odot x_{0}\right)}{|k|}<\delta \\
(\delta=|k| \cdot \epsilon) \Leftrightarrow & d\left(k \odot x, k \odot x_{0}\right)>\Delta-\epsilon,
\end{aligned}
$$

mostrando que neste caso a função é descontínua pela direita. Por outro lado, assuma 
agora que $x<x_{0}$ ( limite pela esquerda ):

$$
\begin{aligned}
d\left(k \odot x, k \odot x_{0}\right) & =d(k \odot x, a) \\
& =|\bmod 2(k x, \Delta, a)-a| \\
& =\left|\left(k x-\left|\frac{k x-a}{\Delta}\right| \Delta\right)-a\right| \\
& =\left|k x-l_{j} \Delta-a\right| \\
& =|k| \cdot\left|x-\frac{a+l_{j} \Delta}{k}\right| \\
(\text { Eq. 1.6.3) } & =|k| \cdot\left|x-x_{j}\right| \\
& =|k| \cdot d\left(x, x_{0}\right)
\end{aligned}
$$

Assim,

$$
\begin{aligned}
& d\left(x, x_{0}\right)<\delta \\
\Leftrightarrow & \frac{d\left(k \odot x, k \odot x_{0}\right)}{|k|}<\delta \\
(\delta=|k| \cdot \epsilon) \Leftrightarrow & d\left(k \odot x, k \odot x_{0}\right)<\epsilon,
\end{aligned}
$$

mostrando que neste caso a função é contínua pela esquerda e completando a demonstração da afirmação iv). Finalmente, suponha que $k<0, x_{0}=x_{n}=a$ e $x>x_{0}$ ( limite pela direita ):

$$
\begin{aligned}
d\left(k \odot x, k \odot x_{0}\right) & =d(k \odot x, a) \\
& =|\bmod 2(k x, \Delta, a)-a| \\
& =\left|\left(k x-\left|\frac{k x-a}{\Delta}\right| \Delta\right)-a\right| \\
& =\left|k x-l_{n-1} \Delta-a\right| \\
& =|k| \cdot\left|x-\frac{a+l_{n-1} \Delta}{k}\right| \\
\left(l_{n}=l_{n-1}+1\right) & =|k| \cdot\left|x-x_{n}+\frac{\Delta}{k}\right|
\end{aligned}
$$




$$
\begin{aligned}
& =|k| \cdot\left(\frac{\Delta}{|k|}-\left|x-x_{n}\right|\right) \\
& =\Delta-|k| \cdot d(x, a) \\
& =\Delta-|k| \cdot d\left(x, x_{0}\right)
\end{aligned}
$$

Assim,

$$
\begin{aligned}
& d\left(x, x_{0}\right)<\delta \\
\Leftrightarrow & \frac{\Delta-d\left(k \odot x, k \odot x_{0}\right)}{|k|}<\delta \\
(\delta=|k| \cdot \epsilon) \Leftrightarrow & d\left(k \odot x, k \odot x_{0}\right)>\Delta-\epsilon,
\end{aligned}
$$

mostrando que neste caso a função é descontínua pela direita e completando a demonstração da afirmação v).

Proposição 1.6.8. Sejam $k, a, \Delta$ e $f$ como na proposição 1.6.7. Então a função $f:\left(I_{[a, \Delta]}, \mathcal{T}_{I_{[a, \Delta]}}\right) \longrightarrow\left(I_{[a, \Delta]}, \mathcal{T}_{I_{[a, \Delta]}}\right)$ é contínua se e somente se uma das seguintes condições se verificar:

i) $k=1$ ou

ii) $k=0$ ou

iii) $\frac{a}{\Delta} \notin \mathbb{Z},|k| \leqslant \operatorname{Fr}\left(-\operatorname{sgn}(k) \frac{(k-1) a}{\Delta}\right), k \in(-1,0) \cup(0,1)$.

iv) $\frac{a}{\Delta} \in \mathbb{Z}, 0<k \leqslant 1$

Demonstração. Se $k=1$, então $f:\left(I_{[a, \Delta]}, \mathcal{T}_{I_{[a, \Delta]}}\right) \longrightarrow\left(I_{[a, \Delta]}, \mathcal{T}_{I_{[a, \Delta]}}\right)$ é a identidade, que é trivialmente contínua, pois a imagem inversa de um conjunto aberto é o próprio conjunto que é aberto pela escolha inicial deste conjunto. Se $k=0$, então $f$ é a função constante igual a 0 , que também é trivialmente contínua, pois a imagem inversa de 
qualquer conjunto contendo 0 é $I_{[a, \Delta]} \in \mathcal{T}_{I_{[a, \Delta]}}$ e a imagem inversa de qualquer conjunto que não contenha 0 é o conjunto vazio $\emptyset \in \mathcal{T}_{I_{[a, \Delta]}}$.

Para os outros casos, pela expressão 1.6.1, a equação $k \odot x=a$ tem tantas soluções quantos forem os valores de $l$ distintos. Seja $m$ o número de números inteiros nos intervalos acima e sejam $I_{1}$ e $I_{m}$ respectivamente o menor e o maior número inteiro no mesmo intervalo considerado. Então é claro que em cada intervalo, $m=I_{m}-I_{1}+1$, e uma análise cuidadosa das desigualdades acima nos permite escrever:

$$
\left\{\begin{array}{l}
k<0 \Rightarrow I_{1}=\left\lfloor\frac{(k-1) a}{\Delta}+k\right\rfloor+1, I_{m}=\left\lfloor\frac{(k-1) a}{\Delta}\right\rfloor \\
k>0 \Rightarrow I_{1}=\left\lceil\frac{(k-1) a}{\Delta}\right\rceil, I_{m}=\left\lceil\frac{(k-1) a}{\Delta}+k\right\rceil-1
\end{array}\right.
$$

Deste modo,

$$
\begin{aligned}
& \left\{\begin{array}{l}
k<0 \Rightarrow m=1+\left\lfloor\frac{(k-1) a}{\Delta}\right\rfloor-\left\lfloor\frac{(k-1) a}{\Delta}+k\right\rfloor-1 \\
k>0 \Rightarrow m=1+\left\lceil\frac{(k-1) a}{\Delta}+k\right\rceil-1-\left\lceil\frac{(k-1) a}{\Delta}\right\rceil
\end{array}\right. \\
& \Leftrightarrow\left\{\begin{array}{l}
k<0 \Rightarrow m=\left\lfloor\frac{(k-1) a}{\Delta}\right\rfloor-\left\lfloor\frac{(k-1) a}{\Delta}\right\rfloor-\lfloor k\rfloor-\left\lfloor\operatorname{Fr}\left(\frac{(k-1) a}{\Delta}\right)+\operatorname{Fr}(k)\right\rfloor \\
k>0 \Rightarrow m=\left\lceil\frac{(k-1) a}{\Delta}\right\rceil+\lceil k\rceil-\left\lfloor\operatorname{Fr}\left(-\frac{(k-1) a}{\Delta}\right)+\operatorname{Fr}(-k)\right\rfloor-\left\lceil\frac{(k-1) a}{\Delta}\right\rceil
\end{array}\right. \\
& \Leftrightarrow\left\{\begin{array}{l}
k<0 \Rightarrow m=-\lfloor k\rfloor-\left\lfloor\operatorname{Fr}\left(\frac{(k-1) a}{\Delta}\right)+\operatorname{Fr}(k)\right\rfloor \\
k>0 \Rightarrow m=-\lfloor-k\rfloor-\left\lfloor\operatorname{Fr}\left(-\frac{(k-1) a}{\Delta}\right)+\operatorname{Fr}(-k)\right\rfloor
\end{array}\right. \\
& \Leftrightarrow\left\{\begin{array}{l}
k<0 \Rightarrow m=-\left\lfloor\operatorname{Fr}\left(\frac{(k-1) a}{\Delta}\right)+\lfloor k\rfloor+\operatorname{Fr}(k)\right\rfloor \\
k>0 \Rightarrow m=-\left\lfloor\operatorname{Fr}\left(-\frac{(k-1) a}{\Delta}\right)+\lfloor-k\rfloor+\operatorname{Fr}(-k)\right\rfloor
\end{array}\right. \\
& \Leftrightarrow\left\{\begin{array}{l}
k<0 \Rightarrow m=-\left\lfloor\operatorname{Fr}\left(\frac{(k-1) a}{\Delta}\right)+k\right\rfloor \\
k>0 \Rightarrow m=-\left\lfloor\operatorname{Fr}\left(-\frac{(k-1) a}{\Delta}\right)-k\right\rfloor
\end{array}\right. \\
& \Leftrightarrow m=-\left\lfloor\operatorname{Fr}\left(-\operatorname{sgn}(k) \frac{(k-1) a}{\Delta}\right)-|k|\right\rfloor
\end{aligned}
$$




$$
=-\lfloor-|k|\rfloor-\left\lfloor\operatorname{Fr}\left(-\operatorname{sgn}(k) \frac{(k-1) a}{\Delta}\right)+\operatorname{Fr}(-|k|)\right\rfloor .
$$

Pela proposição 1.6.7, a função $f$ só pode ser contínua se a equação $k \odot x=a$, $(k \neq 0$ e $k \neq 1)$ não possuir raízes ou se no caso de possuir raiz, ela seja única e igual a $a$ e $k>0$. Continuando a análise da equação acima, tem-se

$$
\begin{aligned}
& m=-\lfloor-|k|\rfloor-\left\lfloor\operatorname{Fr}\left(-\operatorname{sgn}(k) \frac{(k-1) a}{\Delta}\right)+\operatorname{Fr}(-|k|)\right\rfloor \\
& \Leftrightarrow \quad\lceil|k|\rceil-m=\left\lfloor\operatorname{Fr}\left(-\operatorname{sgn}(k) \frac{(k-1) a}{\Delta}\right)+\operatorname{Fr}(-|k|)\right\rfloor \\
& \text { ( Prop.1.4.11- ix) } \Rightarrow \begin{cases}-\operatorname{sgn}(k) \frac{(k-1) a}{\Delta} \notin \mathbb{Z} \mathrm{e}-|k| \notin \mathbb{Z} \quad \Rightarrow 0 \leqslant\lceil|k|\rceil-m \leqslant 1 \\
-\operatorname{sgn}(k) \frac{(k-1) a}{\Delta} \in \mathbb{Z} \text { ou }-|k| \in \mathbb{Z} \Rightarrow\lceil|k|\rceil-m=0\end{cases} \\
& \Leftrightarrow \begin{cases}\frac{(k-1) a}{\Delta} \notin \mathbb{Z} \text { e } k \notin \mathbb{Z} \quad \Rightarrow m \leqslant\lceil|k|\rceil \leqslant m+1 \\
\frac{(k-1) a}{\Delta} \in \mathbb{Z} \text { ou } k \in \mathbb{Z} \quad \Rightarrow\lceil|k|\rceil=m\end{cases} \\
& \Leftrightarrow \begin{cases}\frac{(k-1) a}{\Delta} \notin \mathbb{Z} \text { e } k \notin \mathbb{Z} \quad \Rightarrow\lceil|k|\rceil=m \text { ou }\lceil|k|\rceil=m+1 \\
\frac{(k-1) a}{\Delta} \in \mathbb{Z} \text { e } k \notin \mathbb{Z} \quad \Rightarrow m-1<|k| \leqslant m \\
\frac{(k-1) a}{\Delta} \notin \mathbb{Z} \text { e } k \in \mathbb{Z} \quad \Rightarrow|k|=m\end{cases} \\
& \Leftrightarrow \begin{cases}\frac{(k-1) a}{\Delta} \notin \mathbb{Z} \text { e } k \notin \mathbb{Z} \quad \Rightarrow m-1<|k| \leqslant m+1 \\
\frac{(k-1) a}{\Delta} \in \mathbb{Z} \text { e } k \notin \mathbb{Z} \quad \Rightarrow m-1<|k| \leqslant m \\
\frac{(k-1) a}{\Delta} \notin \mathbb{Z} \text { e } k \in \mathbb{Z} \quad \Rightarrow|k|=m\end{cases} \\
& (m \in \mathbb{Z}) \Leftrightarrow\left\{\begin{array}{l}
\frac{(k-1) a}{\Delta} \notin \mathbb{Z} \text { e } k \notin \mathbb{Z} \quad \Rightarrow m-1<|k|<m+1 \\
\frac{(k-1) a}{\Delta} \in \mathbb{Z} \text { e } k \notin \mathbb{Z} \quad \Rightarrow m-1<|k|<m
\end{array}\right. \\
& \frac{(k-1) a}{\Delta} \notin \mathbb{Z} \text { e } k \in \mathbb{Z} \Rightarrow|k|=m
\end{aligned}
$$


A primeira possibilidade de $f$ ser contínua permitida pela proposição 1.6 .7 é se o número de raízes for zero. De acordo com o desenvolvimento anterior, os valores possíveis de $k$ para cada caso são

$$
\begin{aligned}
& \left\{\begin{array}{l}
\frac{(k-1) a}{\Delta} \notin \mathbb{Z} \text { e } k \notin \mathbb{Z} \quad \Rightarrow-1<|k|<1 \\
\frac{(k-1) a}{\Delta} \in \mathbb{Z} \text { e } k \notin \mathbb{Z} \quad \Rightarrow-1<|k|<0 \\
\frac{(k-1) a}{\Delta} \notin \mathbb{Z} \text { e } k \in \mathbb{Z} \quad \Rightarrow|k|=0
\end{array}\right. \\
& \Leftrightarrow\left\{\begin{array}{l}
\frac{(k-1) a}{\Delta} \notin \mathbb{Z} \text { e } k \notin \mathbb{Z} \quad \Rightarrow 0<|k|<1 \\
\frac{(k-1) a}{\Delta} \in \mathbb{Z} \text { e } k \notin \mathbb{Z} \quad \Rightarrow \nexists k \\
\frac{(k-1) a}{\Delta} \notin \mathbb{Z} \text { e } k \in \mathbb{Z} \quad \Rightarrow \nexists k
\end{array}\right.
\end{aligned}
$$

Conclusão, para $m=0$, a única possibilidade de $f$ ser contínua é $\frac{(k-1) a}{\Delta} \notin \mathbb{Z}$ e $0<|k|<1$. Mas as duas condições anteriores juntas implicam em $0<|k|<1$ e $\frac{a}{\Delta} \notin \mathbb{Z}$. Assim

$$
\begin{aligned}
& 0<|k|<1 \\
\Leftrightarrow & \quad|| k \mid\rceil=1 \\
\Leftrightarrow & \left|\operatorname{Fr}\left(-\operatorname{sgn}(k) \frac{(k-1) a}{\Delta}\right)+\operatorname{Fr}(-|k|)\right|=1 \\
\Leftrightarrow & 1 \leqslant \operatorname{Fr}\left(-\operatorname{sgn}(k) \frac{(k-1) a}{\Delta}\right)+\operatorname{Fr}(-|k|)<2
\end{aligned}
$$

( Prop. 1.4.11-xii), $k \notin \mathbb{Z}$ )

$$
\Leftrightarrow 1-\operatorname{Fr}\left(-\operatorname{sgn}(k) \frac{(k-1) a}{\Delta}\right) \leqslant 1-\operatorname{Fr}(|k|)<2-\operatorname{Fr}\left(-\operatorname{sgn}(k) \frac{(k-1) a}{\Delta}\right)
$$

( Prop. 1.4.11- x) )

$$
\Leftrightarrow \operatorname{Fr}\left(-\operatorname{sgn}(k) \frac{(k-1) a}{\Delta}\right)-1<|k| \leqslant \operatorname{Fr}\left(-\operatorname{sgn}(k) \frac{(k-1) a}{\Delta}\right)
$$

( Prop. 1.4.11- ii)) 


$$
\Leftrightarrow \quad 0<|k| \leqslant \operatorname{Fr}\left(-\operatorname{sgn}(k) \frac{(k-1) a}{\Delta}\right)
$$

O que completa a demonstração do item iii).
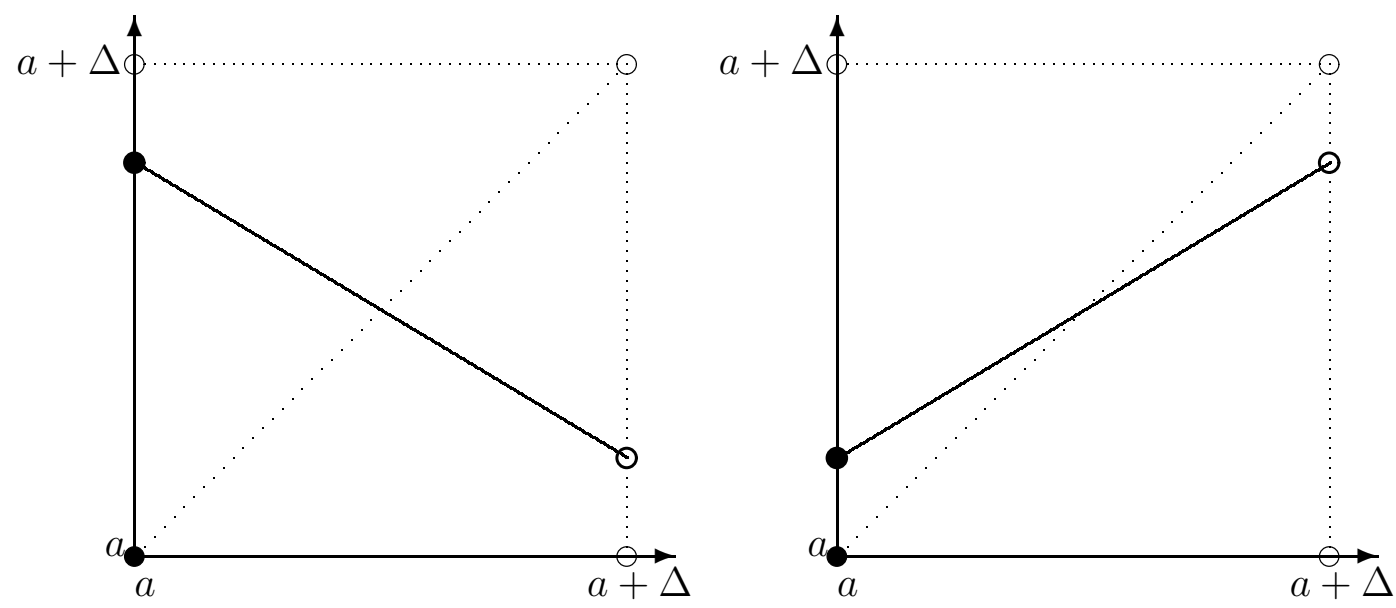

Figura 1.1: Caso $m=0, \frac{a}{\Delta} \notin \mathbb{Z}, k \leqslant 1$

Para mostrar o item iv), analise a outra possibilidade permitida pela proposição 1.6.7, que é a equação possuir uma única raiz $x_{1}=a$ e $k>0$. Usando a relação 1.6.1,

$$
\begin{aligned}
& a=\frac{a+n \Delta}{k} \\
& \Leftrightarrow n=\frac{(k-1) a}{\Delta} \in \mathbb{Z} \\
(\text { Eq. 1.6.6 }) \Leftrightarrow & \frac{(k-1) a}{\Delta} \in \mathbb{Z} \text { e } k \notin \mathbb{Z} \\
\Rightarrow & 0<|k|<1\left(\Rightarrow \frac{a}{\Delta} \in \mathbb{Z}\right) \\
\Leftrightarrow & \lceil|k|\rceil=1 \\
(\text { Eq. 1.6.5 }) & \Rightarrow\left\lfloor\operatorname{Fr}\left(-\operatorname{sgn}(k) \frac{(k-1) a}{\Delta}\right)+\operatorname{Fr}(-|k|)\right\rfloor=0 \\
\left(\frac{a}{\Delta} \in \mathbb{Z} \text { e } k \notin \mathbb{Z}\right) \Rightarrow & \lfloor\operatorname{Fr}(-|k|)\rfloor=0 \\
\Leftrightarrow & 0 \leqslant \operatorname{Fr}(-|k|)<1
\end{aligned}
$$




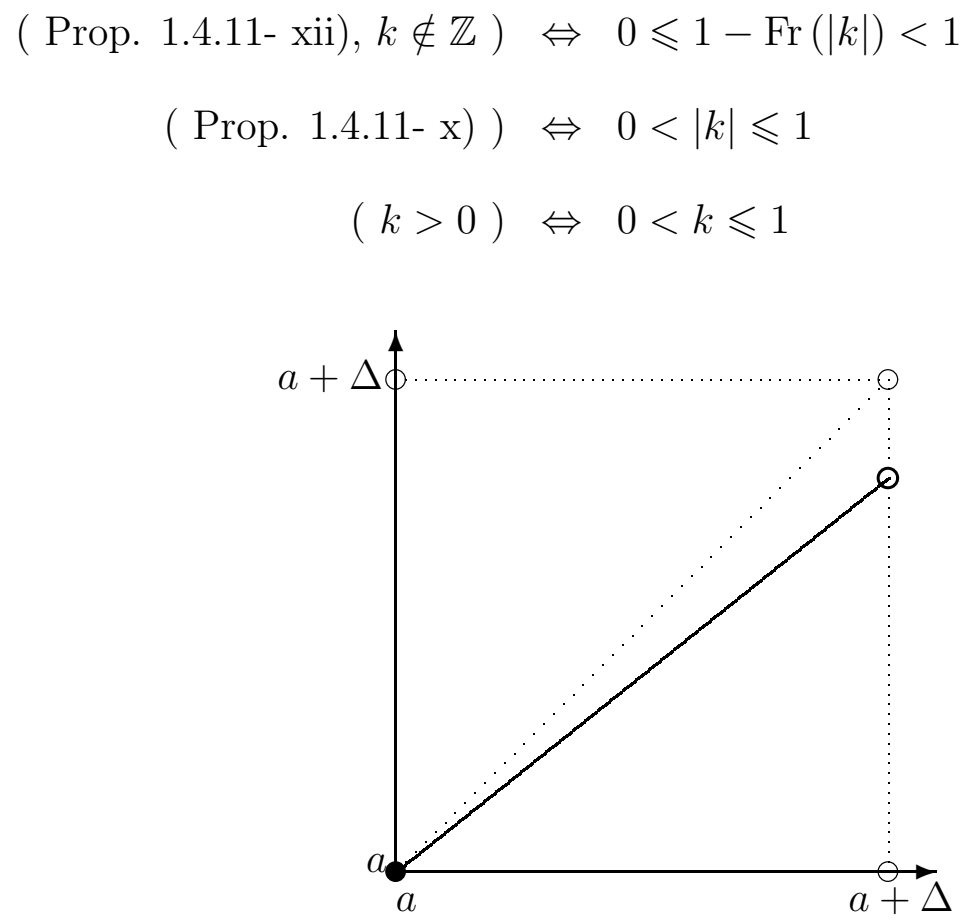

Figura 1.2: Caso $m=1, x_{1}=a, \frac{a}{\Delta} \in \mathbb{Z}, 0<k \leqslant 1$

Proposição 1.6.9. Sejam $k$, a e $\Delta$ como na proposição 1.6 .8 e seja $f$ a seguinte função:

$$
\begin{aligned}
f:\left(I_{[a, \Delta]}, \mathcal{T}_{g_{I}}\right) & \longrightarrow\left(I_{[a, \Delta]}, \mathcal{T}_{g_{I}}\right) \\
x & \longmapsto y=k \odot x .
\end{aligned}
$$

Então a função $f$ é contínua se e somente se $k \in \mathbb{Z}, \forall a \in \mathbb{R}, \Delta \in(0,+\infty)$.

Demonstração. Pelo lema 1.5.12, vale a seguinte relação de inclusão entre as topologias: $\mathcal{T}_{g_{I}} \subset \mathcal{T}_{I_{[a, \Delta]}}$. Porém, não é possível usar o lema 1.5 .13 porque a topologia do co-domínio não é a mesma nos dois casos. A idéia desta demonstração é aproveitar os resultados obtidos na demonstração da proposição 1.6.7. Deste modo, aqui será 
utilizada a mesma notação que na referida proposição. Nos casos em que $k=0$ e $k=1$ mostra-se que $f$ é contínua pelo mesmo argumento topológico utilizado na proposição 1.6.8.

Suponha inicialmente que $x$ e $x_{0} \in L_{j}, j=0, \ldots, n$, e $x_{0} \neq a$. Nestas condições, obteve-se na proposição 1.6 .7 que $d\left(k \odot x, k \odot x_{0}\right)=|k| \cdot d\left(x, x_{0}\right)$, portanto:

$$
\begin{aligned}
& g_{I}\left(k \odot x, k \odot x_{0}\right) \\
= & \min \left\{d\left(k \odot x, k \odot x_{0}\right), \Delta-d\left(k \odot x, k \odot x_{0}\right)\right\} \\
= & \min \left\{|k| \cdot d\left(x, x_{0}\right), \Delta-|k| \cdot d\left(x, x_{0}\right)\right\} \\
= & |k| \cdot d\left(x, x_{0}\right) \\
= & |k| \cdot \min \left\{d\left(x, x_{0}\right), \Delta-d\left(x, x_{0}\right)\right\} \\
= & |k| \cdot g_{I}\left(x, x_{0}\right)
\end{aligned}
$$

Deste modo,

$$
\begin{aligned}
& g_{I}\left(x, x_{0}\right)<\delta \\
\Leftrightarrow & \frac{g_{I}\left(k \odot x, k \odot x_{0}\right)}{|k|}<\delta \\
(\delta=|k| \cdot \epsilon) \Leftrightarrow & g_{I}\left(k \odot x, k \odot x_{0}\right)<\epsilon
\end{aligned}
$$

Mostrando que nestas condições, $f$ é contínua. Suponha agora que $k>0, x=$ $x_{i} \neq a$. Conforme a proposição 1.6.7, se $x>x_{0}$, é possível mostrar novamente que $d\left(k \odot x, k \odot x_{0}\right)=|k| \cdot d\left(x, x_{0}\right)$, e por um desenvolvimento análogo ao anterior, mostrase que a função $f$ é contínua pela direita em $x=x_{i} \neq a$. Nas mesmas condições, se $x<x_{0}$, mostrou-se que $d\left(k \odot x, k \odot x_{0}\right)=\Delta-|k| \cdot d\left(x, x_{0}\right)$, então:

$$
\begin{aligned}
& g_{I}\left(k \odot x, k \odot x_{0}\right) \\
= & \min \left\{d\left(k \odot x, k \odot x_{0}\right), \Delta-d\left(k \odot x, k \odot x_{0}\right)\right\}
\end{aligned}
$$




$$
\begin{aligned}
& =\min \left\{\Delta-|k| \cdot d\left(x, x_{0}\right), \Delta-\left(\Delta-|k| \cdot d\left(x, x_{0}\right)\right)\right\} \\
& =\min \left\{\Delta-|k| \cdot d\left(x, x_{0}\right),|k| \cdot d\left(x, x_{0}\right)\right\} \\
& =|k| \cdot d\left(x, x_{0}\right) \\
& =|k| \cdot \min \left\{d\left(x, x_{0}\right), \Delta-d\left(x, x_{0}\right)\right\} \\
& =|k| \cdot g_{I}\left(x, x_{0}\right)
\end{aligned}
$$

Com esse resultado e por um desenvolvimento idêntico a equação 1.6.7, mostra-se que $f$ também é contínua pela esquerda em $x_{0}=x_{i} \neq a$, o que significa que $f$ é contínua neste ponto. Suponha agora que $k<0, x=x_{i} \neq a$. Utilizando os resultados da proposição 1.6.7 e o mesmo desenvolvimento feito acima, mostra-se que $f$ é contínua.

A diferença agora é que na topologia da métrica $g_{I}$, é possível se aproximar do ponto $x_{0}=a$ pela esquerda. Portanto, o que falta ser analisado é o ponto $x_{0}=a$ quando $x_{i}=a$ nos limites pela direita e pela esquerda e $x_{0}=a$ quando $x_{i} \neq a$ nos limites pela esquerda pois neste caso, nos limites pela direita já se mostrou que $f$ é contínua.

Utilizando os resultados da proposição 1.6.7 e os desenvolvimentos anteriores nos casos dos limites pela direita quando $x_{0}=x_{i}=a$ e, $k>0$ ou $k<0$, mostra-se que $f$ é contínua pela direita em $x_{0}=x_{i}=a$.

Até o presente momento, está provado que $f$ é contínua à direita em todos os pontos e contínua a esquerda em todos os pontos, à excessão talvez do ponto $x_{0}=a$. Uma vez que $f$ é contínua pela direita em $x_{0}=a$, para que $f$ seja contínua no ponto $x_{0}=a$ é necessário que:

$$
\begin{aligned}
\lim _{x \rightarrow a^{-}} f(x) & =f(a) \Leftrightarrow \\
\lim _{\gamma \rightarrow 0} f(a+\Delta-\gamma) & =f(a) \Leftrightarrow
\end{aligned}
$$




$$
\begin{aligned}
\lim _{\gamma \rightarrow 0} \bmod 2(k(a+\Delta-\gamma), \Delta, a) & =\bmod 2(k a, \Delta, a) \Leftrightarrow \\
\bmod 2(k(a+\Delta), \Delta, a) & =\bmod 2(k a, \Delta, a) \Leftrightarrow \\
k(a+\Delta)-\left\lfloor\frac{k(a+\Delta)-a}{\Delta}\right\rfloor \Delta & =k a-\left\lfloor\frac{k a-a}{\Delta}\right\rfloor \Delta \Leftrightarrow \\
k \Delta-\left\lfloor\frac{(k-1) a}{\Delta}+k\right\rfloor \Delta & =-\left\lfloor\frac{(k-1) a}{\Delta}\right\rfloor \Delta \Leftrightarrow \\
k-\left\lfloor\frac{(k-1) a}{\Delta}\right\rfloor-\lfloor k\rfloor-\left\lfloor\operatorname{Fr}\left(\frac{(k-1) a}{\Delta}\right)+\operatorname{Fr}(k)\right\rfloor & =-\left\lfloor\frac{(k-1) a}{\Delta}\right\rfloor \Leftrightarrow \\
\left\lfloor\operatorname{Fr}\left(\frac{(k-1) a}{\Delta}\right)+\operatorname{Fr}(k)\right\rfloor & =\operatorname{Fr}(k) \Rightarrow \\
\operatorname{Fr}(k) \in \mathbb{Z} \Rightarrow \operatorname{Fr}(k)=0 \Rightarrow k \in \mathbb{Z} &
\end{aligned}
$$

Corolario 1.6.10. Continuidade de $\oplus e \odot$. As operações $\oplus:\left(\mathbb{G}_{\Delta}, g\right) \times\left(\mathbb{G}_{\Delta}, g\right) \longrightarrow$ $\left(\mathbb{G}_{\Delta}, g\right) e \odot: \mathbb{R} \times\left(\mathbb{G}_{\Delta}, g\right) \longrightarrow\left(\mathbb{G}_{\Delta}, g\right)$ são contínuas nas mesmas condições das proposições 1.6 .5 e 1.6.9.

Demonstração. As proposições 1.6.5 e 1.6.9 junto com o fato de os espaços $\left(I_{[a, \Delta]}, g_{I}\right)$ e $\left(\mathbb{G}_{\Delta}, g\right)$ serem homeomorfos, implica em que as operações $\oplus$ e $\odot$ também são contínuas nas mesmas condições destas proposições ([32]-3.10.2).

\section{$1.7 \quad \sigma$-álgebra de sub-conjuntos de $\mathbb{G}_{\Delta}$}

Antes de definir as variáveis aleatórias cíclicas, falta resolver apenas um detalhe técnico com relação à $\sigma$-álgebra a ser utilizada. Até o presente momento, existem duas candidatas à $\sigma$-álgebra de sub-conjuntos de $\mathbb{G}_{\Delta}$. A primeira é a $\sigma$-álgebra de Borel gerada a partir da topologia gerada pela métrica $g$ no conjunto $\mathbb{G}_{\Delta}$, isto é: 
Definição 1.7.1. $\sigma$-álgebra de Borel em $\mathbb{G}_{\Delta}$ derivada da métrica g.

$$
\Sigma_{\mathbb{G}_{\Delta}} \equiv \operatorname{Borel}\left(\mathcal{T}_{\mathbb{G}_{\Delta}}\right)
$$

Note que

$$
\Sigma_{\mathbb{G}_{\Delta}} \equiv \operatorname{Borel}\left(\mathcal{T}_{\mathbb{G}_{\Delta}}\right) \equiv \Pi_{[a, \Delta]}^{-1}\left(\operatorname{Borel}\left(\mathcal{T}_{g_{I}}\right)\right) \equiv \Pi_{[a, \Delta]}^{-1}\left(\Sigma_{g_{I}}\right)
$$

A segunda candidata a $\sigma$-álgebra é aquela gerada pela $\sigma$-álgebra de Borel gerada pela topologia $\mathcal{T}_{[a, \Delta]}$ através do homeomorfismo $\Pi_{[a, \Delta]}^{-1}$, ou seja:

Definição 1.7.2. $\sigma$-álgebra de Borel em $\mathbb{G}_{\Delta}$ importada de $I_{[a, \Delta]}$.

$$
\Sigma_{\mathbb{G}_{\Delta}}^{\prime} \equiv \Pi_{[a, \Delta]}^{-1}\left(\operatorname{Borel}\left(\mathcal{T}_{I_{[a, \Delta]}}\right)\right)
$$

Cada uma destas duas $\sigma$-álgebras tem suas vantagens. Se $\Sigma_{\mathbb{G}_{\Delta}}$ for utilizada, funções contínuas serão automaticamente mensuráveis, e isto é muito conveniente, sendo inclusive o motivo pelo qual se usam as $\sigma$-álgebras de Borel. Se $\Sigma_{\mathbb{G}_{\Delta}}^{\prime}$ for utilizada, todo resultado válido para uma variável aleatória real definida em $\left(I_{[a, \Delta]}, \Sigma_{I_{[a, \Delta]}}\right)$ pode ser facilmente transportado para $\left(\mathbb{G}_{\Delta}, \Sigma_{\mathbb{G}_{\Delta}}^{\prime}\right)$ através do mapeamento $\Pi_{[a, \Delta]}$ e sua inversa.

Foi mostrado no lema 1.5.12 que $\Pi_{[a, \Delta]}\left(\mathcal{T}_{\mathbb{G}_{\Delta}}\right)=\mathcal{T}_{g_{I}} \subset \mathcal{T}_{I_{[a, \Delta]}}$ e que $\mathcal{T}_{g_{I}} \neq \mathcal{T}_{I_{[a, \Delta]}}$. Dada esta relação de inclusão, é um resultado trivial que $\operatorname{Borel}\left(\mathcal{T}_{g_{I}}\right) \subset \operatorname{Borel}\left(\mathcal{T}_{[a, \Delta]}\right)$. Porém, o ideal é que fosse válida a relação $\operatorname{Borel}\left(\mathcal{T}_{g_{I}}\right)=\operatorname{Borel}\left(\mathcal{T}_{[a, \Delta]}\right)$, ou de modo equivalente aplicando $\Pi_{[a, \Delta]}^{-1}$, que $\Sigma_{\mathbb{G}_{\Delta}}=\Sigma_{\mathbb{G}_{\Delta}}^{\prime}$ pois neste caso seria possível se usufruir das vantagens de ambas as $\sigma$-álgebras.

Como o objeto deste estudo é um espaço métrico, a definição [32]-3.9.4 permite a caracterização de uma topologia através do sistema de vizinhanças locais. O argumento a ser desenvolvido aqui é o seguinte: a topologia $\mathcal{T}_{I_{[a, \Delta]}}$ será analisada em 
termos das vizinhanças locais que a geram e desta forma será possível mostrar que a topologia $\mathcal{T}_{[a, \Delta]}$ é composta por uma parte que gera a topologia $\mathcal{T}_{g_{I}}$ e por outra parte que gera a diferença entre as topologias $\mathcal{T}_{I_{[a, \Delta]}}$ e $\mathcal{T}_{g_{I}}$. Então será mostrado que as vizinhanças locais que geram essa diferença entre as topologias pertencem à $\sigma$-álgebra de Borel gerada por $\mathcal{T}_{g_{I}}$. Deste modo ficará provado que $\operatorname{Borel}\left(\mathcal{T}_{g_{I}}\right)=\operatorname{Borel}\left(\mathcal{T}_{\left.I_{[a, \Delta]}\right]}\right)$.

Definição 1.7.3. Conjunto fechado. Seja $\left(X, \mathcal{T}_{X}\right)$ um espaço topológico. Um conjunto $A \subset X$ é fechado se o conjunto $A^{\prime}=X-A$ for aberto.

Lema 1.7.4. Seja $\left(X, \mathcal{T}_{X}\right)$ um espaço topológico. Se o conjunto A é fechado, então $A \in \operatorname{Borel}\left(\mathcal{T}_{X}\right)$.

Demonstração.

$A$ é fechado

( Def. 1.7.3) $\Leftrightarrow A^{\prime}$ é aberto

( Def. 1.3.4) $\Leftrightarrow \quad A^{\prime} \in \operatorname{Borel}\left(\mathcal{T}_{X}\right)$

( Def. 1.3.2) $\Leftrightarrow \quad A^{\prime \prime} \in \operatorname{Borel}\left(\mathcal{T}_{X}\right)$

$\left(A^{\prime \prime}=A\right) \quad \Leftrightarrow \quad A \in \operatorname{Borel}\left(\mathcal{T}_{X}\right)$

Definição 1.7.5. Vizinhanças locais abertas e fechadas. Seja $(X, d)$ um espaço métrico e seja $r \in(0,+\infty)$. Uma vizinhança local aberta de raio $r$ do ponto $x_{0} e ́$ o conjunto

$$
N_{r}\left(x_{0}\right)=\left\{x \in X \mid d\left(x_{0}, x\right)<r\right\}
$$

e uma vizinhança local fechada de raio $r$ do ponto $x_{0}$ é o conjunto

$$
N_{r}\left[x_{0}\right]=\left\{x \in X \mid d\left(x_{0}, x\right) \leqslant r\right\} .
$$


Quando for necessário referir-se a uma vizinhança local independente do fato de ser aberta ou fechada, será utilizada a notação $N_{r}\{x\}$.

Definição 1.7.6. Definição alternativa de conjunto aberto (vide [32]-3.9.4). Um subconjunto $A$ de um espaço métrico $(X, d)$ é aberto se e somente se $A$ contém uma vizinhança local ( aberta ou fechada) de todos os seus pontos, isto é

$$
\text { A é aberto } \left.\Leftrightarrow \forall x \in A, \exists r \in(0,+\infty) \mid N_{r}\{x\}\right) \subset A \text {. }
$$

Lema 1.7.7. Expansão de conjuntos abertos. Todo conjunto aberto num espaço métrico pode ser escrito como a união de vizinhanças locais, isto é

$$
\text { A é aberto } \Leftrightarrow A=\bigcup_{x}^{x \in A} N_{r_{x}}\{x\}, r_{x} \in(0,+\infty)
$$

Demonstração.

$$
\begin{aligned}
A \text { é aberto } & \Leftrightarrow \forall x \in A, \exists N_{r_{x}}\{x\} \mid N_{r_{x}}\{x\} \subset A \\
& \Leftrightarrow A=\bigcup_{x} N_{r_{x}}\{x\}
\end{aligned}
$$

No caso de $I_{[a, \Delta]}$ a notação $N_{r}\{x\}$ será utilizada para as vizinhanças locais geradas pela métrica $d$, enquanto a notação $M_{r}\{x\}$ será utilizada para as vizinhanças locais geradas pela métrica $g_{I}$.

Proposição 1.7.8. $M_{r}(x)$ é aberto na topologia $\mathcal{T}_{I_{[a, \Delta]}}$, isto é

$$
M_{r}(x) \in \mathcal{T}_{I_{[a, \Delta]}}
$$

Demonstração. Como $M_{r}(x)$ é aberto em $\mathcal{T}_{g_{I}}$, e o lema 1.5.12 implica em $\mathcal{T}_{g_{I}} \subset \mathcal{T}_{I_{[a, \Delta]}}$, isto significa que necessariamente $M_{r}(x) \in \mathcal{T}_{I_{[a, \Delta]}}$. 
Lema 1.7.9. Se existe $x^{\prime} \in M_{r}\left(x_{0}\right)$ tal que $\Delta-d\left(x_{0}, x^{\prime}\right) \leqslant d\left(x_{0}, x^{\prime}\right)$, então a $\in M_{r}\left(x_{0}\right)$.

Demonstração.

$$
\begin{aligned}
& \exists x^{\prime} \in M_{r}\left(x_{0}\right) \mid \Delta-d\left(x_{0}, x^{\prime}\right) \leqslant d\left(x_{0}, x^{\prime}\right) \\
& \Leftrightarrow \exists x^{\prime} \in M_{r}\left(x_{0}\right) \mid \Delta / 2 \leqslant d\left(x_{0}, x^{\prime}\right) \\
& \text { (Proposição 1.5.3) } \Leftrightarrow \exists x^{\prime} \in M_{r}\left(x_{0}\right) \mid \Delta / 2 \leqslant d\left(x_{0}, x^{\prime}\right)<\Delta \\
& \Leftrightarrow \exists x^{\prime} \in M_{r}\left(x_{0}\right) \mid 0<\Delta-d\left(x_{0}, x^{\prime}\right) \leqslant \Delta / 2
\end{aligned}
$$

Note que como $a=\inf _{x \in I_{[a, \Delta]}}\{x\}$, tem-se que $\forall x \in I_{[a, \Delta]}, d(x, a)=x-a$. Calcule então:

$$
\begin{aligned}
& g_{I}\left(x_{0}, a\right)+g_{I}\left(a, x^{\prime}\right) \\
= & \min \left\{d\left(x_{0}, a\right), \Delta-d\left(x_{0}, a\right)\right\}+\min \left\{d\left(x^{\prime}, a\right), \Delta-d\left(x^{\prime}, a\right)\right\} \\
= & \min \left\{x_{0}-a, \Delta-x_{0}+a\right\}+\min \left\{x^{\prime}-a, \Delta-x^{\prime}+a\right\} \\
= & \min \left\{x_{0}-a+x^{\prime}-a, \Delta-\left(x^{\prime}-x_{0}\right), \Delta-\left(x_{0}-x^{\prime}\right), 2 \Delta-\left(x_{0}-a\right)-\left(x^{\prime}-a\right)\right\} .
\end{aligned}
$$

Existem dois casos a se considerar: $x^{\prime}<x_{0}$ e $x^{\prime}>x_{0}$. Porém a expressão acima é simétrica em relação à troca de $x^{\prime}$ por $x_{0}$, portanto, basta analisar um dos casos e o resultado da análise vale para ambos os casos. Suponha então que $x^{\prime}<x_{0}$.

i) $x_{0}-a+x^{\prime}-a$ :

$$
\begin{aligned}
& d\left(x_{0}, x^{\prime}\right) \geqslant \Delta / 2 \\
\Leftrightarrow & x_{0}-x^{\prime} \geqslant \Delta / 2 \\
\Leftrightarrow & x_{0} \geqslant x^{\prime}+\Delta / 2 \\
\Leftrightarrow & x_{0}-a \geqslant x^{\prime}-a+\Delta / 2
\end{aligned}
$$




$$
\begin{aligned}
& \Leftrightarrow \quad x_{0}-a+x^{\prime}-a \geqslant x^{\prime}-a+x^{\prime}-a+\Delta / 2 \\
& \Leftrightarrow \quad x_{0}-a+x^{\prime}-a \geqslant \Delta / 2
\end{aligned}
$$

ii) $\Delta-\left(x^{\prime}-x_{0}\right)$ :

$$
\begin{gathered}
\left(x^{\prime}-x_{0}\right)<0 \\
\Leftrightarrow \Delta-\left(x^{\prime}-x_{0}\right)>\Delta
\end{gathered}
$$

iii) $\Delta-\left(x_{0}-x^{\prime}\right)$ :

$$
\Delta-\left(x_{0}-x^{\prime}\right) \leqslant \Delta / 2
$$

iv) $\Delta-\left(x_{0}-a\right)+\Delta-\left(x^{\prime}-a\right)$ :

$$
\begin{aligned}
& d\left(x_{0}, x^{\prime}\right) \geqslant \Delta / 2 \\
\Leftrightarrow & x_{0}-x^{\prime} \geqslant \Delta / 2 \\
\Leftrightarrow & -x^{\prime} \geqslant-x_{0}+\Delta / 2 \\
\Leftrightarrow & a+\Delta-x^{\prime} \geqslant a+\Delta-x_{0}+\Delta / 2 \\
\Leftrightarrow & a+\Delta-x^{\prime}+a+\Delta-x_{0} \geqslant a+\Delta-x_{0}+a+\Delta-x_{0}+\Delta / 2 \\
\Leftrightarrow & \Delta-\left(x_{0}-a\right)+\Delta-\left(x^{\prime}-a\right) \geqslant \Delta / 2
\end{aligned}
$$

Como quando $x^{\prime}<x_{0}$ o mínimo é $\Delta-\left(x_{0}-x^{\prime}\right)$, no caso $x^{\prime}>x_{0}$ o mínimo será $\Delta-\left(x^{\prime}-x_{0}\right)$, assim pode-se escrever de uma forma geral que o mínimo é $\Delta-d\left(x_{0}, x^{\prime}\right)$.

Portanto:

$$
\begin{aligned}
& g_{I}\left(x_{0}, a\right)+g_{I}\left(a, x^{\prime}\right) \\
= & \Delta-d\left(x_{0}, x^{\prime}\right)
\end{aligned}
$$




$$
\begin{aligned}
(\text { Por hipótese }) & =\min \left\{d\left(x_{0}, x^{\prime}\right), \Delta-d\left(x_{0}, x^{\prime}\right)\right\} \\
& =g_{I}\left(x_{0}, x^{\prime}\right) .
\end{aligned}
$$

E assim:

$$
\begin{aligned}
& g_{I}\left(x_{0}, a\right)+g_{I}\left(a, x^{\prime}\right)=g_{I}\left(x_{0}, x\right)<r \\
\left(g_{I}\left(a, x^{\prime}\right)=r^{\prime}\right) \Leftrightarrow & g_{I}\left(x_{0}, a\right)+r^{\prime}<r \\
\left(r^{\prime}>0\right) \Leftrightarrow & g_{I}\left(x_{0}, a\right)<r-r^{\prime}<r
\end{aligned}
$$

(Definição de $\left.M_{r}\left(x_{0}\right)\right) \Leftrightarrow a \in M_{r}\left(x_{0}\right)$.

Corolario 1.7.10. Se $a \notin M_{r}\left(x_{0}\right)$ então $\forall x \in M_{r}\left(x_{0}\right), \Delta-d\left(x_{0}, x\right)>d\left(x_{0}, x\right)$.

Demonstração. Este corolário nada mais é do que a contrapositiva do lema 1.7.9.

Lema 1.7.11. Sejam $x_{0}$ e $r_{0}$ tais que $a \notin N_{r_{0}}\left(x_{0}\right)$. Então $\exists x_{1}$ e $\exists r_{1}$ tais que $N_{r_{0}}\left(x_{0}\right)=$ $M_{r_{1}}\left(x_{1}\right)$.

Demonstração. Seja $s=\sup _{x \in N_{r_{0}}\left(x_{0}\right)}\{x\}$ e $i=\inf _{x \in N_{r_{0}}\left(x_{0}\right)}\{x\}$ e faça $x_{1}=(s+i) / 2$ e $r_{1}=(s-i) / 2$. Então,

$$
N_{r_{0}}\left(x_{0}\right)=\left\{x \in I_{[a, \Delta]} \mid d\left(x_{0}, x\right)<r_{0}\right\}
$$

(Definição de $\left.x_{1}, r_{1}\right) \Leftrightarrow N_{r_{0}}\left(x_{0}\right)=\left\{x \in I_{[a, \Delta]} \mid d\left(x_{1}, x\right)<r_{1}\right\}$

(Corolário 1.7.10) $\Leftrightarrow N_{r_{0}}\left(x_{0}\right)=\left\{x \in I_{[a, \Delta]} \mid \min \left\{d\left(x_{1}, x\right), \Delta-d\left(x_{1}, x\right)\right\}<r_{1}\right\}$

(Definição de $\left.g_{I}\right) \Leftrightarrow N_{r_{0}}\left(x_{0}\right)=\left\{x \in I_{[a, \Delta]} \mid g_{I}\left(x_{1}, x\right)<r_{1}\right\}$

(Definição de $\left.M_{r}(x)\right) \Leftrightarrow N_{r_{0}}\left(x_{0}\right)=M_{r_{1}}\left(x_{1}\right)$ 
Lema 1.7.12. Seja $A=N_{r}[a]$ e $a+r<\Delta$. Então existem $x_{1}$ e $r_{1}$ tais que $A^{\prime}=$ $N_{r_{1}}\left(x_{1}\right)$.

Demonstração.

$$
\begin{aligned}
& A=\left\{x \in I_{[a, \Delta]} \mid d(x, a) \leqslant r\right\} \\
\Leftrightarrow \quad & A^{\prime}=\left\{x \in I_{[a, \Delta]} \mid d(x, a)>r\right\}
\end{aligned}
$$

(Proposição 1.5.3) $\Leftrightarrow A^{\prime}=\left\{x \in I_{[a, \Delta]} \mid r<d(x, a)<\Delta\right\}$

$$
\begin{aligned}
\left(a=\inf _{x \in I_{[a, \Delta]}}\{x\}\right) & \Leftrightarrow A^{\prime}=\left\{x \in I_{[a, \Delta]} \mid r<x-a<\Delta\right\} \\
& \Leftrightarrow A^{\prime}=\left\{x \in I_{[a, \Delta]} \mid-\Delta<a-x<-r\right\} \\
\left(+\frac{\Delta+r}{2}\right) & \Leftrightarrow A^{\prime}=\left\{x \in I_{[a, \Delta]} \mid-\frac{\Delta-r}{2}<a+\frac{\Delta+r}{2}-x<\frac{\Delta-r}{2}\right\}
\end{aligned}
$$

Fazendo $x_{1}=a+(\Delta+r) / 2$ e $r_{1}=(\Delta-r) / 2$ tem-se:

$$
\begin{aligned}
& A^{\prime}=\left\{x \in I_{[a, \Delta]} \mid-r_{1}<x_{1}-x<r_{1}\right\} \\
\Leftrightarrow & A^{\prime}=\left\{x \in I_{[a, \Delta]}|| x_{1}-x \mid<r_{1}\right\} \\
\Leftrightarrow & A^{\prime}=\left\{x \in I_{[a, \Delta]} \mid d\left(x_{1}, x\right)<r_{1}\right\} \\
\Leftrightarrow & A^{\prime}=N_{r_{1}}\left(x_{1}\right)
\end{aligned}
$$

Lema 1.7.13. O conjunto $N_{r}[a]$ é fechado na topologia $\mathcal{T}_{g_{I}}$.

Demonstração.

$$
\begin{aligned}
& N_{r}^{\prime}[a] \\
(\text { Lema 1.7.12) }= & N_{r_{1}}\left(x_{1}\right) \\
(\text { Lema 1.7.11) }= & M_{r_{2}}\left(x_{2}\right)
\end{aligned}
$$


Portanto, o complemento de $N_{r}[a]$ é um conjunto aberto na topologia $\mathcal{T}_{g_{I}}$, logo pela definição 1.7.3, $N_{r}[a]$ é fechado na topologia $\mathcal{T}_{g_{I}}$.

Lema 1.7.14. Seja $A \in \mathcal{T}_{I_{[a, \Delta]}}$ tal que a $\notin A$. Então $A \in \mathcal{T}_{g_{I}}$.

Demonstração.

$$
\begin{aligned}
& A \in \mathcal{T}_{I_{[a, \Delta]}}, a \notin A \\
&(\text { Lema 1.7.7) }= \bigcup_{x}^{x \in A} N_{r_{x}}(x), a \notin N_{r_{x}}(x) \\
&(\text { Lema 1.7.11) }= \bigcup_{x^{\prime}}^{x^{\prime} \in A} M_{r_{x^{\prime}}}\left(x^{\prime}\right), a \notin M_{r_{x^{\prime}}}\left(x^{\prime}\right) \\
& \text { (Definição 1.7.6) } \Leftrightarrow A \in \mathcal{T}_{g_{I}}, a \notin A .
\end{aligned}
$$

Lema 1.7.15. Seja $A \in \mathcal{T}_{I_{[a, \Delta]}}$. Então A pode ser escrito da forma $A=B \cup C$, onde $B \in \mathcal{T}_{g_{I}} e$

$$
C= \begin{cases}\emptyset, & \text { se } a \notin A \\ N_{r}[a], & \text { se } a \in A\end{cases}
$$

Demonstração. Se $a \notin A$, então faça $B=A$ e $C=\emptyset$. Então pelo lema 1.7.14, $B \in \mathcal{T}_{g_{I}}$. Se $a \in A$, então a definição 1.7 .6 implica em que seja possível escrever $A=B U N_{r}[a]$. Tome então $B=A-N_{r}[a]$ e $C=N_{r}[a]$. Falta mostrar que $A-N_{r}[a]$ é aberto em $\mathcal{T}_{g_{I}}$. Para isto, note que $I_{[a, \Delta]}-N_{r}[a]$ é um conjunto aberto em $\mathcal{T}_{I_{[a, \Delta]}}$ e que

$$
\begin{aligned}
& \left(I_{[a, \Delta]}-N_{r}[a]\right) \cap A \\
= & \left(I_{[a, \Delta]}-N_{r}[a]\right) \cap\left[\left(A-N_{r}[a]\right) \cup N_{r}[a]\right]
\end{aligned}
$$




$$
\begin{aligned}
& =\left[\left(I_{[a, \Delta]}-N_{r}[a]\right) \cap\left(A-N_{r}[a]\right)\right] \cup\left[\left(I_{[a, \Delta]}-N_{r}[a]\right) \cap N_{r}[a]\right] \\
& =\left[\left(I_{[a, \Delta]}-N_{r}[a]\right) \cap\left(A-N_{r}[a]\right)\right] \cup \emptyset \\
& =A-N_{r}[a]
\end{aligned}
$$

Assim, $A-N_{r}[a]$ pode ser escrito como a interseção de dois conjuntos abertos, o que significa que $A-N_{r}[a]$ é aberto em $\mathcal{T}_{I_{[a, \Delta]}}$. Como $a \notin A-N_{r}[a]$, mais uma vez pelo lema 1.7.14, é possível concluir que $A-N_{r}[a] \in \mathcal{T}_{g_{I}}$.

Lema 1.7.16. Seja $A \in \mathcal{T}_{I_{[a, \Delta]}}$. Então $A \in \operatorname{Borel}\left(\mathcal{T}_{g_{I}}\right)$.

Demonstração.

$$
\begin{aligned}
& A \in \mathcal{T}_{I_{[a, \Delta]}} \\
(\text { Lema 1.7.15, Lema 1.7.12) } \Rightarrow & A=B \cup C, B \in \mathcal{T}_{g_{I}} \text { e } C^{\prime} \in \mathcal{T}_{g_{I}} \\
(\text { Definição 1.3.2) } \Leftrightarrow & A \in \operatorname{Borel}\left(\mathcal{T}_{g_{I}}\right)
\end{aligned}
$$

Teorema 1.7.17. $\Sigma_{\mathbb{G}_{\Delta}}=\Sigma_{\mathbb{G}_{\Delta}}^{\prime}$

Demonstração. Conforme já foi comentado, é uma consequência trivial do lema 1.5.12 que $\Sigma_{\mathbb{G}_{\Delta}}^{\prime} \subset \Sigma_{\mathbb{G}_{\Delta}}$. Pelo lema 1.7.16 $\mathcal{T}_{I_{[a, \Delta]}} \subset \operatorname{Borel}\left(\mathcal{T}_{g_{I}}\right)$. Portanto tem-se necessariamente que $\Sigma_{\mathbb{G}_{\Delta}} \subset \Sigma_{\mathbb{G}_{\Delta}}^{\prime}$. As duas relações de inclusão anteriores implicam em $\Sigma_{\mathbb{G}_{\Delta}}=\Sigma_{\mathbb{G}_{\Delta}}^{\prime}$

\subsection{Variáveis Aleatórias em $\left(\mathbb{G}_{\Delta}, \oplus, \odot, g\right)$}

A discussão a seguir visa buscar uma relação entre variáveis aleatórias reais e variáveis aleatórias cíclicas de modo que seja possível trabalhar ora com uma, ora 
com a outra, principalmente a fim de aproveitar todo o conhecimento já disponível sobre variáveis aleatórias reais.

Definição 1.8.1. Variável Aleatória Cíclica. Sejam $\Omega$ um conjunto, $\Sigma$ uma $\sigma$-álgebra de sub-conjuntos de $\Omega$ e $\mu$ uma medida de probabilidade. Então uma função mensurável $f:(\Omega, \Sigma, \mu) \longrightarrow\left(\mathbb{G}_{\Delta}, \Sigma_{\mathbb{G}_{\Delta}}\right)$ será chamada de variável aleatória cíclica.

Para a seguinte proposição, considere o diagrama da figura 1.3.

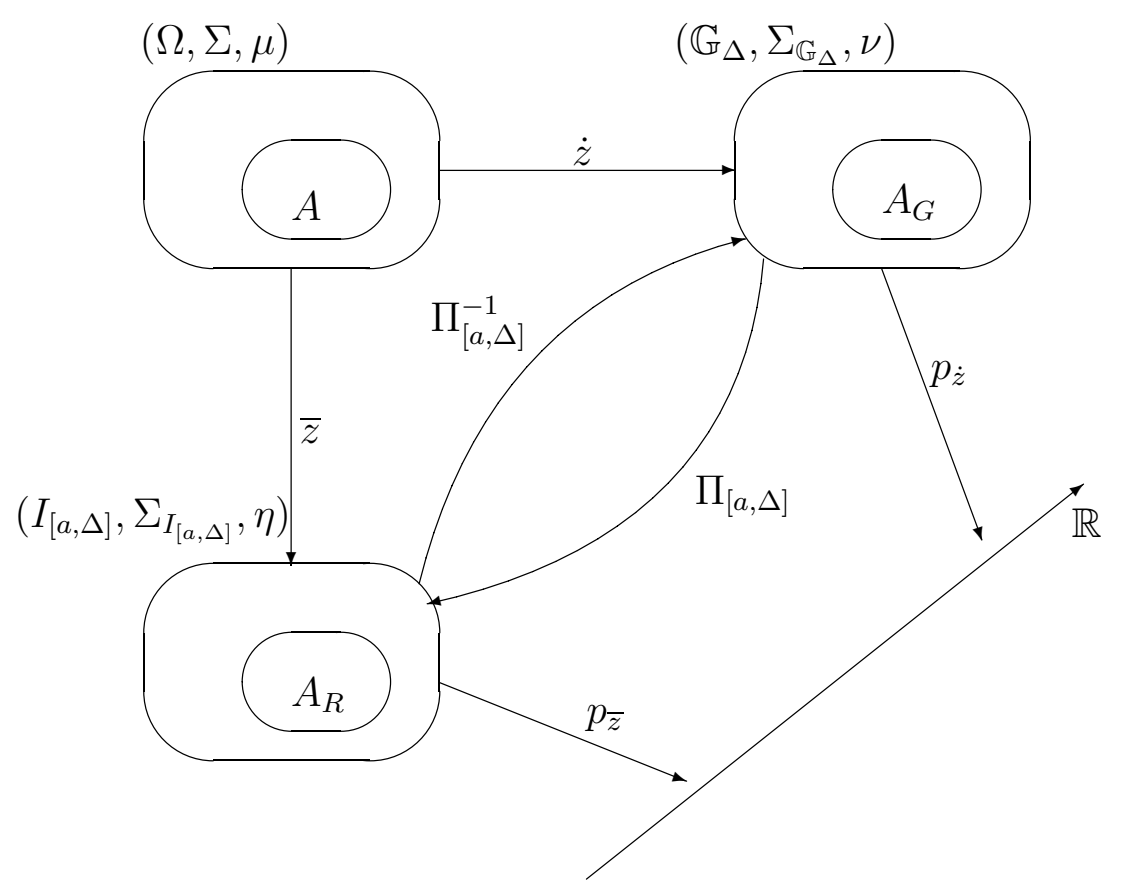

Figura 1.3: Diagrama dos conjuntos

Proposição 1.8.2. Variável aleatória cíclica induzida. Seja z uma variável aleatória real, conforme a ilustração e sejam $\prod_{[a, \Delta]}$ e $\Pi_{[a, \Delta]}^{-1}$ os mapeamentos estudados nas seções anteriores. Então a expressão

$$
\dot{z}=\Pi_{[a, \Delta]}^{-1} \circ \bar{z}
$$

define uma variável aleatória cíclica. 
Demonstração. Tudo que tem que ser mostrado é que $\dot{z}$ é mensurável. Note que a definição da variável aleatória cíclica $\dot{z}$ implica em ([19]-10)

$$
\dot{z}^{-1}=\bar{z}^{-1} \circ \Pi_{[a, \Delta]}
$$

Seja então $A_{R} \subset I_{[a, \Delta]}, A_{R} \in \Sigma_{I_{[a, \Delta]}}$. Este conjunto determina um outro conjunto $A_{G} \subset \mathbb{G}_{\Delta}$ através do mapeamento $\Pi_{[a, \Delta]}^{-1}$. Seja então $\Sigma_{\mathbb{G}_{\Delta}}^{\prime}=\Pi_{[a, \Delta]}^{-1}\left(\Sigma_{I_{[a, \Delta]}}\right)$. Pelo teorema 1.7.17, sabe-se que $\Sigma_{\mathbb{G}_{\Delta}}^{\prime}=\Sigma_{\mathbb{G}_{\Delta}}=\operatorname{Borel}\left(\mathcal{T}_{\mathbb{G}_{\Delta}}\right)$. Desta forma, a função $\dot{z}$ definida acima é mensurável, pois

$$
\begin{aligned}
& A_{G} \in \Sigma_{\mathbb{G}_{\Delta}} \\
\left(\text { Definição de } \Sigma_{\mathbb{G}_{\Delta}}\right) \Leftrightarrow & A_{R}=\Pi_{[a, \Delta]}\left(A_{G}\right) \in \Sigma_{I_{[a, \Delta]}} \\
\text { (Mensurabilidade de } \bar{z}) & \Leftrightarrow A=\bar{z}^{-1}\left(A_{R}\right)=\bar{z}^{-1}\left(\Pi_{[a, \Delta]}\left(A_{G}\right)\right) \in \Sigma \\
\text { (Eq. 1.8.2) } & \Leftrightarrow A=\dot{z}^{-1}\left(A_{G}\right) \in \Sigma
\end{aligned}
$$

Sendo $\bar{z}$ e $\dot{z}$ funções mensuráveis, conforme visto numa seção anterior, a medida $\mu$ induz duas novas medidas

$$
\left\{\begin{array}{l}
\eta\left(A_{R}\right)=\mu\left(\bar{z}^{-1}\left(A_{R}\right)\right)=\mu(A) \\
\nu\left(A_{G}\right)=\mu\left(\dot{z}^{-1}\left(A_{G}\right)\right)=\mu(A)
\end{array}\right.
$$

As duas expressões acima implicam que as novas medidas $\eta$ e $\nu$ que foram induzidas possuem o mesmo valor quando calculadas em elementos correspondentes segundo o mapeamento $\Pi_{[a, \Delta]}$ e sua inversa.

A definição a seguir cria o análogo da medida de Lebesgue em $\left(\mathbb{G}_{\Delta}, \Sigma_{\mathbb{G}_{\Delta}}\right)$. A partir desta definição será possível falar de "função densidade de probabilidade" em $\mathbb{G}_{\Delta}$. 
Definição 1.8.3. Medida de Lebesgue em $\mathbb{G}_{\Delta}$. Seja $\Lambda$ a medida de Lebesgue em $\mathbb{R}$ e $\operatorname{seja} A \in \Sigma_{\mathbb{G}_{\Delta}}$. Então a função

$$
\begin{aligned}
\Lambda_{\mathbb{G}_{\Delta}}: \Sigma_{\mathbb{G}_{\Delta}} & \longrightarrow \mathbb{R} \\
A & \longmapsto \Lambda\left(\Pi_{[a, \Delta]}(A)\right)
\end{aligned}
$$

será chamada de medida de Lebesgue em $\mathbb{G}_{\Delta}$.

Portanto, se a medida $\eta$ for absolutamente contínua em relação à medida de Lebesgue, a medida $\nu$ também será absolutamente contínua em relação à medida de Lebesgue em $\mathbb{G}_{\Delta}$, e o teorema de Radon-Nikodým ([2]-8.9) garante a existência de funções $p_{\bar{z}}$ e $p_{\dot{z}}$, tais que

$$
\left\{\begin{array}{l}
\eta\left(A_{R}\right)=\int_{A_{R}} p_{\bar{z}} d \Lambda \\
\nu\left(A_{G}\right)=\int_{A_{G}} p_{\dot{z}} d \Lambda_{\mathbb{G}_{\Delta}}
\end{array}\right.
$$

A função $p_{\bar{z}}$ é a função densidade de probabilidade da variável aleatória real $\bar{z}$. De modo análogo, para as variáveis aleatórias cíclicas, a função $p_{\dot{z}}$ será chamada de "função densidade de probabilidade cíclica".

Seja $z$ uma variável aleatória real, e seja $\bar{z}$ uma outra variável aleatória real definida por $\bar{z}=\bmod 2(z, \Delta, a)$. O co-domínio de $\bar{z}$ é $I_{[a, \Delta]} \subset \mathbb{R}$. Supondo que a medida de probabilidade da variável aleatória $z$ seja absolutamente contínua em relação à medida de Lebesgue, e seja $p_{z}(\alpha) \in M^{+}\left(\mathbb{R}, \Sigma_{\mathbb{R}}\right)$ tal que $\left\|p_{z}(\alpha)\right\|_{1}=1$, a função densidade de probabilidade de z. Então,

Proposição 1.8.4. A função densidade de probabilidade de $\bar{z}=\bmod 2(z, \Delta, a)$ é dada por $p_{\bar{z}}(\alpha)=\sum_{n=-\infty}^{\infty} p_{z}(\alpha+n \Delta), \alpha \in I_{[a, \Delta]}$. 
Demonstração.

$$
\begin{aligned}
p_{\bar{z}}(\alpha) & =\frac{d}{d \alpha} \mathbf{P}[\bar{z} \leqslant \alpha]=\frac{d}{d \alpha} \mathbf{P}\left[z \in \bigcup_{n=-\infty}^{\infty}[a+n \Delta, \alpha+n \Delta)\right] \\
& =\frac{d}{d \alpha} \sum_{n=-\infty}^{\infty} \mathbf{P}[z \in[a+n \Delta, \alpha+n \Delta)]=\frac{d}{d \alpha} \sum_{n=-\infty}^{\infty} \int_{a+n \Delta}^{\alpha+n \Delta} p_{z}(\beta) d \beta \\
& =\sum_{n=-\infty}^{\infty} \frac{d}{d \alpha} \int_{a+n \Delta}^{\alpha+n \Delta} p_{z}(\beta) d \beta=\sum_{n=-\infty}^{\infty} p_{z}(\alpha+n \Delta)
\end{aligned}
$$

Proposição 1.8.5. A função $p_{\bar{z}}: \mathbb{R} \longrightarrow \mathbb{R}$, quando estendida para todos os números reais, é periódica com período $\Delta$.

Demonstração.

$$
\begin{aligned}
p_{\bar{z}}(\alpha+m \Delta) & =\sum_{n=-\infty}^{\infty} p_{z}(\alpha+m \Delta+n \Delta) \\
& =(\text { fazendo } j=m+n) \\
& =\sum_{j=-\infty}^{\infty} p_{z}(\alpha+j \Delta)=p_{\bar{z}}(\alpha), \forall m \in \mathbb{Z}
\end{aligned}
$$

Graças a essa periodicidade, a função $p_{\bar{z}}(\alpha)$ possui o mesmo valor em todos os elementos de $\mathbb{R}$ que pertencem a uma mesma classe de equivalência $[\alpha]$. Qualquer elemento da classe de equivalência pode ser usado para avaliar o valor da função. Então, é possível definir uma função com domínio em $\mathbb{G}_{\Delta}$, através do homeomorfismo $\Pi_{[a, \Delta]}$ da seguinte forma:

$$
p_{\dot{z}}(\dot{\alpha})=p_{\bar{z}}\left(\Pi_{[a, \Delta]}(\dot{\alpha})\right)
$$

onde $\dot{z}=[\bar{z}]$ é uma variável aleatória cíclica. Porém, a proposição 1.8.4 vale apenas quando a medida da variável aleatória real for absolutamente contínua em relação à 
medida de Lebesgue. Nem sempre será desejável partir de uma variável aleatória real para então definir uma variável aleatória cíclica correspondente. Desta forma, para definir corretamente de forma geral este novo tipo de variável aleatória é necessário antes definir a sua $\sigma$-álgebra de eventos para que se possa falar de uma medida em $\mathbb{G}_{\Delta}$.

O homeomorfismo $\Pi_{[a, \Delta]}: \mathbb{G}_{\Delta} \longrightarrow I_{[a, \Delta]}$ cria uma relação de um para um inversível entre os conjuntos $\mathbb{G}_{\Delta}$ e $I_{[a, \Delta]} \subset \mathbb{R}$ e também entre os conjuntos $\Sigma_{\mathbb{G}_{\Delta}}$ e $\Sigma_{I_{[a, \Delta]}}$. Como a relação é inversível, a medida escolhida em $\left(I_{[a, \Delta]}, \Sigma_{I_{[a, \Delta]}}\right)$ pode ser usada para determinar a medida em $\left(\mathbb{G}_{\Delta}, \Sigma_{\mathbb{G}_{\Delta}}\right)$, conforme a expressão 1.3.1. A relação 1.8.4 pode então ser usada para relacionar uma variável aleatória real com uma variável aleatória cíclica, sempre que a medida da variável aleatória real for absolutamente contínua em relação à medida de Lebesgue. De forma análoga ao caso das variáveis aleatórias reais, pode-se usar a notação da função delta de Dirac para representar a parte singular da medida de variáveis aleatórias cíclicas em suas funções densidade de probabilidade.

Lema 1.8.6. Seja $f:\left(\mathbb{G}_{\Delta}, \Sigma_{\mathbb{G}_{\Delta}}, \nu\right) \longrightarrow\left(\mathbb{R}, \Sigma_{\mathbb{R}}\right)$ uma função mensurável e $\Lambda_{\mathbb{G}_{\Delta}}$ conforme a definição 1.8.3. Então:

$$
\int_{A} f d \Lambda_{\mathbb{G}_{\Delta}}=\int_{\Pi_{[a, \Delta]}(A)} f \circ \Pi_{[a, \Delta]}^{-1} d \Lambda
$$

Demonstração. A demonstração é mera aplicação da definição 1.8.3.

$$
\begin{aligned}
\int_{A} f d \Lambda_{\mathbb{G}_{\Delta}} & =\int_{A} f d\left(\Lambda \circ \Pi_{[a, \Delta]}\right) \\
& =\int_{A} f \circ \Pi_{[a, \Delta]}^{-1} \circ \Pi_{[a, \Delta]} d\left(\Lambda \circ \Pi_{[a, \Delta]}\right) \\
& =\int_{\Pi_{[a, \Delta]}(A)} f \circ \Pi_{[a, \Delta]}^{-1} d \Lambda
\end{aligned}
$$


O lema 1.8.6 permite, sempre que for conveniente, calcular uma integral no espaço $\mathbb{G}_{\Delta}$ através de uma integral equivalente no intervalo $I_{[a, \Delta]}$, que é um subconjunto dos números reais. De qualquer modo, o importante é que a medida de Lebesgue em $\mathbb{G}_{\Delta}$ está definida, mesmo que em função da medida de Lebesgue em $I_{[a, \Delta]}$. Assim, a partir de agora será usado o mesmo abuso de notação encontrado em qualquer livro de engenharia que consiste em expressões do tipo

$$
\int_{A} f d \Lambda_{\mathbb{G}_{\Delta}}=\int_{A} f(\dot{x}) d \dot{x}, A \in \Sigma_{\mathbb{G}_{\Delta}}
$$

Teorema 1.8.7. Sejam $f: \mathbb{G}_{\Delta} \longrightarrow \mathbb{R}$ e $g: \mathbb{R} \longrightarrow \mathbb{R}$ duas funções tais que $f=$ $g \circ \prod_{[a, \Delta]} \cdot$ Então

$$
\int_{A} f(\dot{x}) d \dot{x}=\int_{\Pi_{[a, \Delta]}(A)} g(x) d x
$$

Demonstração.

$$
\begin{aligned}
& \int_{A} f(\dot{x}) d \dot{x} \\
(\text { Equação 1.8.5) } & =\int_{A} f d \Lambda_{\mathbb{G}_{\Delta}} \\
(\text { Lema 1.8.6) } & =\int_{\Pi_{[a, \Delta]}(A)} f \circ \Pi_{[a, \Delta]}^{-1} d \Lambda \\
(\text { Definição de } f) & =\int_{\Pi_{[a, \Delta]}(A)} g \circ \Pi_{[a, \Delta]} \circ \Pi_{[a, \Delta]}^{-1} d \Lambda \\
\left(\Pi_{[a, \Delta]} \circ \Pi_{[a, \Delta]}^{-1}=\text { Identidade }\right) & =\int_{\Pi_{[a, \Delta]}(A)} g d \Lambda \\
(\text { Equação 1.8.5) } & =\int_{\Pi_{[a, \Delta]}(A)} g(x) d x
\end{aligned}
$$


Lema 1.8.8. Medida de Probabilidade em $\mathbb{G}_{\Delta}$. Seja $f: \mathbb{G}_{\Delta} \longrightarrow \mathbb{R}$ uma função mensurável tal que

$$
\int_{\mathbb{G}_{\Delta}} f d \Lambda_{\mathbb{G}_{\Delta}}=1
$$

e seja

$$
\xi(A)=\int_{A} f d \Lambda_{\mathbb{G}_{\Delta}}
$$

Então, $\xi$ é uma medida de probabilidade em $\mathbb{G}_{\Delta}$.

Demonstração. Em [2]-Corolário 4.9, mostra-se que $\xi$ é uma medida, e a condição $\xi\left(\mathbb{G}_{\Delta}\right)=\int_{\mathbb{G}_{\Delta}} f d \Lambda_{\mathbb{G}_{\Delta}}=1$, implica em que $\xi$ é uma medida de probabilidade.

A toda medida de probabilidade em $\left(\mathbb{G}_{\Delta}, \Sigma_{\mathbb{G}_{\Delta}}\right)$ pode ser associada uma variável aleatória $\dot{X}$ com função distribuição de probabilidade igual a

$$
F(\dot{x})=P[\dot{X}<\dot{x}]=\xi([\dot{a}, \dot{x}))=\int_{\dot{a}}^{\dot{x}} f d \Lambda_{\mathbb{G}_{\Delta}}=\int_{\dot{a}}^{\dot{x}} f(\dot{t}) d \dot{t},
$$

onde $\dot{a}=\Pi_{[a, \Delta]}^{-1}(a)$ e $f \in M^{+}\left(\mathbb{G}_{\Delta}, \Sigma_{\mathbb{G}_{\Delta}}\right)$ é a função densidade de probabilidade da variável aleatória $\dot{X}$.

\subsection{Resultados Fundamentais}

Sejam $x$ e $y$ variáveis aleatórias reais, onde $z=x+y$ e $p_{x y}(\alpha, \beta) \in M^{+}\left(\mathbb{R}^{2}, \Sigma_{\mathbb{R}^{2}}\right)$ é a densidade de probabilidade conjunta de $x$ e $y$. Então é um resultado conhecido da teoria de variáveis aleatórias reais (e.g. [34]) que a densidade de probabilidade da variável aleatória real z é dada por

$$
p_{z}(\alpha)=\int_{-\infty}^{\infty} p_{x y}(\beta, \alpha-\beta) d \beta=\int_{-\infty}^{\infty} p_{x y}(\alpha-\beta, \beta) d \beta .
$$


Sejam então $\bar{x}=\bmod 2(x, \Delta, a)$ e $\bar{y}=\bmod 2(y, \Delta, a)$. Usando um desenvolvimento completamente análogo ao da expressão (1.8.3), mostra-se que

$$
\begin{aligned}
p_{\bar{x} y}(\alpha, \beta) & =\sum_{n=-\infty}^{\infty} p_{x y}(\alpha+n \Delta, \beta) \\
p_{x \bar{y}}(\alpha, \beta) & =\sum_{n=-\infty}^{\infty} p_{x y}(\alpha, \beta+n \Delta) \\
p_{\overline{x y}}(\alpha, \beta) & =\sum_{n=-\infty}^{\infty} \sum_{m=-\infty}^{\infty} p_{x y}(\alpha+n \Delta, \beta+m \Delta) .
\end{aligned}
$$

Estas funções são periódicas nas variáveis com barra. Por um argumento igual ao usado na equação (1.8.4), estas mesmas funções podem ser estendidas para variáveis cíclicas da seguinte forma:

$$
\begin{aligned}
p_{\dot{x} y}(\dot{\alpha}, \beta) & =p_{\bar{x} y}\left(\Pi_{[a, \Delta]}(\dot{\alpha}), \beta\right) \\
p_{x \dot{y}}(\alpha, \dot{\beta}) & =p_{x \bar{y}}\left(\alpha, \Pi_{[a, \Delta]}(\dot{\beta})\right) \\
p_{\dot{x} \dot{y}}(\dot{\alpha}, \dot{\beta}) & =p_{\overline{x y}}\left(\Pi_{[a, \Delta]}(\dot{\alpha}), \Pi_{[a, \Delta]}(\dot{\beta})\right) .
\end{aligned}
$$

Proposição 1.9.1. Seja $\bar{z}=\bar{x}+\bar{y}$. A função densidade de probabilidade $p_{\bar{z}}(\alpha)$ pode ser escrita como

$$
p_{\bar{z}}(\alpha)=\int_{-\infty}^{\infty} p_{\bar{x} y}(\alpha-\beta, \beta) d \beta=\int_{-\infty}^{\infty} p_{x \bar{y}}(\beta, \alpha-\beta) d \beta .
$$

Demonstração.

$$
\begin{aligned}
p_{\bar{z}}(\alpha) & = \\
(\text { Eq.1.8.3 }) & =\sum_{n=-\infty}^{\infty} p_{z}(\alpha+n \Delta) \\
(\text { Eq.1.9.1 }) & =\sum_{n=-\infty}^{\infty} \int_{-\infty}^{\infty} p_{x y}(\alpha+n \Delta-\beta, \beta) d \beta=\sum_{n=-\infty}^{\infty} \int_{-\infty}^{\infty} p_{x y}(\beta, \alpha+n \Delta-\beta) d \beta \\
\left(\begin{array}{c}
\text { veja } \\
\text { adiante }
\end{array}\right) & =\int_{-\infty}^{\infty} \sum_{n=-\infty}^{\infty} p_{x y}(\alpha+n \Delta-\beta, \beta) d \beta=\int_{-\infty}^{\infty} \sum_{n=-\infty}^{\infty} p_{x y}(\beta, \alpha+n \Delta-\beta) d \beta
\end{aligned}
$$


$\left(\begin{array}{l}\text { Eq.1.9.2 } \\ \text { Eq.1.9.3 }\end{array}\right)=\int_{-\infty}^{\infty} p_{\bar{x} y}(\alpha-\beta, \beta) d \beta=\int_{-\infty}^{\infty} p_{x \bar{y}}(\beta, \alpha-\beta) d \beta$

A comutatividade do somatório com a integral pode ser justificada se a integral for encarada como um operador linear $\mathbf{I}: \mathbf{L}_{1}\left(\mathbb{R}^{2}\right) \longrightarrow \mathbf{L}_{1}(\mathbb{R})$. Tem-se que ([32]-5.8.2)

$$
\|I\|=\sup _{\|p\|=1}\|I p\|=\sup _{\|p\|=1}\left\|\int p(\alpha, \beta) d \beta\right\|=\sup _{\|p\|=1}\left\|\int p^{\prime}(\alpha)\right\|=\sup _{\|p\|=1} \int\left|p^{\prime}(\alpha)\right| d \alpha=1 .
$$

Ou seja, como $\|\mathbf{I}\|<\infty$, I é um operador linear limitado, o que implica em que I é um operador linear contínuo ([32]-5.6.4). Deste modo, I preserva séries convergentes ([32]-5.6.2), o que implica na comutatividade do somatório com o operador I.

Com o auxílio das relações desenvolvidas até agora, é possível provar o seguinte teorema da soma de variáveis aleatórias cíclicas:

Teorema 1.9.2. Sejam $\dot{x}$ e $\dot{y}$ duas variáveis aleatórias cíclicas e seja $\dot{z}=\dot{x} \oplus \dot{y}$. Então a função densidade de probabilidade de $\dot{z}$ é dada por

$$
p_{\dot{z}}(\dot{\alpha})=\int_{\mathbb{G}_{\Delta}} p_{\dot{x} \dot{y}}(\dot{\gamma}, \dot{\alpha} \ominus \dot{\gamma}) d \dot{\gamma}=\int_{\mathbb{G}_{\Delta}} p_{\dot{x} \dot{y}}(\dot{\alpha} \ominus \dot{\gamma}, \dot{\gamma}) d \dot{\gamma}
$$

Demonstração. Usando na segunda parte da igualdade da proposição (1.9.1) a relação

$$
1(\beta)=\sum_{j=-\infty}^{\infty} G_{[a+j \Delta, a+(j+1) \Delta)}(\beta)=\sum_{j=-\infty}^{\infty} G_{[a, a+\Delta)}(\beta-j \Delta)
$$

Onde $G_{[a, b)}(x)$ é a função constante e igual a 1 no intervalo $[a, b)$, e zero fora deste intervalo, obtém-se o seguinte resultado:

$$
\begin{aligned}
p_{\bar{z}}(\alpha) & =\int_{-\infty}^{\infty} p_{x \bar{y}}(\beta, \alpha-\beta) \sum_{j=-\infty}^{\infty} G_{[a, a+\Delta)}(\beta-j \Delta) d \beta \\
(\gamma=\beta-j \Delta) & =\int_{-\infty}^{\infty} \sum_{j=-\infty}^{\infty} p_{x \bar{y}}(\gamma+j \Delta, \alpha-(\gamma+j \Delta)) G_{[a, a+\Delta)}(\gamma) d \gamma
\end{aligned}
$$




$$
\begin{aligned}
(\text { Prop.1.8.5) } & =\int_{-\infty}^{\infty} \sum_{j=-\infty}^{\infty} p_{x \bar{y}}(\gamma+j \Delta, \alpha-\gamma) G_{[a, a+\Delta)}(\gamma) d \gamma \\
(\text { Eq.1.9.4) } & =\int_{[a, a+\Delta)} p_{\overline{x y}}(\gamma, \alpha-\gamma) d \gamma
\end{aligned}
$$

Usando agora o mapeamento $\Pi_{[a, \Delta]}$, pode-se estender $p_{\bar{z}}(\alpha)$ para $\mathbb{G}_{\Delta}$ :

$$
\begin{aligned}
p_{\dot{z}}(\dot{\alpha}) & = \\
\text { Eq.1.8.4) } & =p_{\bar{z}}\left(\Pi_{[a, \Delta]}(\dot{\alpha})\right) \\
\text { Eq.1.9.11) } & =\int_{I_{[a, \Delta]}} p_{\overline{x y}}\left(\gamma, \Pi_{[a, \Delta]}(\dot{\alpha})-\gamma\right) d \gamma \\
(\text { Teorema 1.8.7) } & =\int_{\mathbb{G}_{\Delta}} p_{\overline{x y}}\left(\Pi_{[a, \Delta]}(\dot{\gamma}), \Pi_{[a, \Delta]}(\dot{\alpha})-\Pi_{[a, \Delta]}(\dot{\gamma})\right) d \dot{\gamma} \\
\text { (Prop. 1.8.5) } & =\int_{\mathbb{G}_{\Delta}} p_{\overline{x y}}\left(\Pi_{[a, \Delta]}(\dot{\gamma}), \Pi_{[a, \Delta]}(\dot{\alpha})-\Pi_{[a, \Delta]}(\dot{\gamma})+k \Delta\right) d \dot{\gamma} \\
(\text { Prop. 1.4.17), } & =\int_{\mathbb{G}_{\Delta}} p_{\overline{x y}}\left(\Pi_{[a, \Delta]}(\dot{\gamma}), \Pi_{[a, \Delta]}(\dot{\alpha} \ominus \dot{\gamma})\right) d \dot{\gamma} \\
\left.\frac{\left.\Pi_{[a, \Delta]}(\dot{x})-\Pi_{[a, \Delta]}(\dot{t})\right]}{\Delta}\right] & \int_{\mathbb{G}_{\Delta}} p_{\dot{x} \dot{y}}(\dot{\gamma}, \dot{\alpha} \ominus \dot{\gamma}) d \dot{\gamma}
\end{aligned}
$$

A segunda parte da igualdade que se deseja mostrar pode ser provada através de um desenvolvimento análogo se no começo da argumentação for utilizada a primeira parte da proposição 1.9 .1 ao invés da segunda.

A expressão (1.9.9) mostra que a densidade de probabilidade da variável $\dot{z}$ está relacionada com as densidades de probabilidade das variáveis $\dot{x}$ e $\dot{y}$ através de uma operação em $\mathbb{G}_{\Delta}$ muito semelhante em forma à expressão que relaciona as variáveis aleatórias $x, y$ e $z=x+y$ no conjunto dos números reais.

No caso em que $x$ e $y$ são variáveis aleatórias independentes, é um resultado imediato que $\bar{x}$ e $\bar{y}$, e consequentemente $\dot{x}$ e $\dot{y}$, também serão independentes, e deste modo tem-se: 
Corolario 1.9.3. Convolução em $\mathbb{G}_{\Delta}$. Sejam $\dot{x}$ e $\dot{y}$ duas variáveis aleatórias cíclicas independentes. Então a função densidade de probabilidade da variável aleatória cíclica $\dot{z}=\dot{x} \oplus \dot{y}$ é dada por:

$$
p_{\dot{z}}(\dot{\alpha})=\int_{\mathbb{G}_{\Delta}} p_{\dot{x}}(\dot{\beta}) p_{\dot{y}}(\dot{\alpha} \ominus \dot{\beta}) d \dot{\beta}=\int_{\mathbb{G}_{\Delta}} p_{\dot{x}}(\dot{\alpha} \ominus \dot{\beta}) p_{\dot{y}}(\dot{\beta}) d \dot{\beta}
$$

Demonstração. Basta usar o teorema (1.9.2) e fazer $p_{\dot{x} \dot{y}}(\dot{\alpha}, \dot{\beta})=p_{\dot{x}}(\dot{\alpha}) p_{\dot{y}}(\dot{\beta})$.

A fim de prosseguir, será necessária a utilização de derivadas em $\mathbb{G}_{\Delta}$, e isto merece um comentário. Como as topologias $\mathcal{T}_{\mathbb{G}_{\Delta}}$ e $\Pi_{[a, \Delta]}^{-1}\left(\mathcal{T}_{I_{[a, \Delta]}}\right)$ não são idênticas, sempre que uma função for derivável na topologia $\mathcal{T}_{\mathbb{G}_{\Delta}}=\Pi_{[a, \Delta]}^{-1}\left(\mathcal{T}_{g_{I}}\right)$, ela também o será na topologia $\Pi_{[a, \Delta]}^{-1}\left(\mathcal{T}_{I_{[a, \Delta]}}\right)$, devido à relação $\mathcal{T}_{g_{I}} \subset \mathcal{T}_{I_{[a, \Delta]}}$, mas a recíproca não é necessariamente verdadeira. Para que a função $f:\left(\mathbb{G}_{\Delta}, \mathcal{T}_{\mathbb{G}_{\Delta}}\right) \longrightarrow\left(R, \mathcal{T}_{\mathbb{R}}\right)$ seja derivável, é necessário que a função $g=\prod_{[a, \Delta]} \circ f:\left(I_{[a, \Delta]}, \mathcal{I}_{[a, \Delta]}\right) \longrightarrow\left(R, \mathcal{T}_{\mathbb{R}}\right)$ seja derivável e que $\lim _{x \rightarrow a+\Delta} f^{\prime}(x)=f^{\prime}(a)$ na topologia $\mathcal{T}_{I_{[a, \Delta]}}$.

Corolario 1.9.4. Sejam $\dot{x}$ e $\dot{y}$ duas variáveis aleatórias cíclicas independentes e seja $\dot{x}$ uma variável aleatória cíclica uniforme. Então a variável aleatória cíclica $\dot{z}=\dot{x} \oplus \dot{y}$ é uniforme e independente de $\dot{y}$.

Demonstração.

i) Uniformidade:

$$
p_{\dot{z}}(\dot{\alpha})=\int_{\mathbb{G}_{\Delta}} p_{\dot{x}}(\dot{\alpha} \ominus \dot{\beta}) p_{\dot{y}}(\beta) d \dot{\beta}=\frac{1}{\Delta} \int_{\mathbb{G}_{\Delta}} p_{\dot{y}}(\dot{\beta}) d \dot{\beta}=\frac{1}{\Delta}
$$

ii) Independência:

$$
\begin{aligned}
{\left[\dot{z} \mid \dot{y}=Y_{0}\right] } & =\dot{x} \oplus Y_{0} \\
\Rightarrow p_{\dot{z} \mid \dot{y}=Y_{0}}(\dot{\alpha}) & =p_{\dot{x} \oplus Y_{0}}(\dot{\alpha})
\end{aligned}
$$




$$
\begin{aligned}
& =\left.\frac{p_{\dot{x}}(\dot{\beta})}{\left|\frac{d}{d \dot{\beta}}\left(\dot{\beta} \oplus Y_{0}\right)\right|}\right|_{\dot{\beta}=\dot{\alpha} \ominus Y_{0}} \\
& =p_{\dot{x}}\left(\dot{\alpha} \ominus Y_{0}\right) \\
& =\frac{1}{\Delta}=p_{\dot{z}}(\dot{\alpha})
\end{aligned}
$$

Note que no corolário anterior não é verdade em geral que $\dot{z}$ seja independente de $\dot{x}$, a menos que $\dot{y}$ também seja uniforme, pois

$$
p_{\dot{z} \mid \dot{x}=X_{0}}(\dot{\alpha})=p_{\dot{y}}\left(\dot{\alpha} \ominus X_{0}\right)
$$

Note ainda que, mesmo que $\dot{x}$ e $\dot{y}$ sejam ambas uniformes, $\dot{z}$ não é independente de $\dot{x}$ e $\dot{y}$ simultaneamente, pois

$$
p_{\dot{z} \mid \dot{x}=X_{0}, \dot{y}=Y_{0}}(\dot{\alpha})=\delta\left(\dot{\alpha} \ominus\left(X_{0} \oplus Y_{0}\right)\right) \text {. }
$$

Corolario 1.9.5. Nas mesmas condições do corolário 1.9.4, a variável aleatória cíclica $\dot{w}=\dot{x} \ominus \dot{y}$ é uniforme e independente de $\dot{y}$.

Demonstração. Faça $\dot{v}=-\dot{y}$. Pela definição da operação $\ominus, \dot{w}=\dot{x} \oplus \dot{v}$. Pelo corolário 1.9.4, $\dot{w}$ é uniforme e independente de $\dot{v}$, consequentemente, $\dot{w}$ é independente de $\dot{y}$.

Teorema 1.9.6. Generalização do Teorema 1.9.2. Sejam $\dot{x}_{1}, \ldots, \dot{x}_{n} n$ variáveis aleatórias cíclicas, e seja $\dot{z}=\dot{x}_{1} \oplus \ldots \oplus \dot{x}_{n}$. Seja também $p_{\dot{x}_{1}, \ldots, \dot{x}_{n}}\left(\dot{\alpha}_{1}, \ldots, \dot{\alpha}_{n}\right)$ a função densidade de probabilidade conjunta das variáveis $\dot{x}_{1}, \ldots, \dot{x}_{n}$. Então a função densidade de probabilidade da variável cíclica ḋ é dada por

$p_{\dot{z}}(\dot{\beta})=$ 


$$
\begin{aligned}
& \int_{\mathbb{G}_{\Delta}} \ldots \int_{\mathbb{G}_{\Delta}} p_{\dot{x}_{1}, \ldots, \dot{x}_{n}}\left(\dot{\alpha}_{1}, \ldots, \dot{\alpha}_{i-1}, \dot{\beta} \ominus \dot{\alpha}_{1} \ominus \ldots \ominus \dot{\alpha}_{i-1} \ominus \dot{\alpha}_{i+1} \ldots \ominus \dot{\alpha}_{n}, \dot{\alpha}_{i+1}, \ldots, \dot{\alpha}_{n}\right) \\
& d \dot{\alpha}_{1} \ldots d \dot{\alpha}_{i-1}, d \dot{\alpha}_{i+1}, d \dot{\alpha}_{n}
\end{aligned}
$$

Demonstração. Considere o seguinte sistema de equações:

$$
\left\{\begin{array} { l l } 
{ \dot { z } _ { 1 } } & { = \dot { x } _ { 1 } } \\
{ } & { \vdots } \\
{ \dot { z } _ { i - 1 } } & { = \dot { x } _ { i - 1 } } \\
{ \dot { z } \quad } & { \dot { x } _ { 1 } \oplus \ldots \oplus \dot { x } _ { i - 1 } \oplus } \\
{ } & { \dot { x } _ { i + 1 } \oplus \ldots \oplus \dot { x } _ { n } } \\
{ \dot { z } _ { i + 1 } } & { = \dot { x } _ { i + 1 } } \\
{ } & { \vdots } \\
{ \dot { z } _ { n } } & { = \dot { x } _ { n } }
\end{array} \Rightarrow \left\{\begin{array}{ll}
\dot{x}_{1} & =\dot{z}_{1} \\
& \vdots \\
\dot{x}_{i-1} & =\dot{z}_{i-1} \\
\dot{x}_{i} & =\dot{z} \ominus \dot{z}_{1} \ldots \ominus \dot{z}_{i-1} \ominus \\
& \dot{z}_{i+1} \ominus \ldots \ominus \dot{z}_{n} \\
\dot{x}_{i+1} & =\dot{z}_{i+1} \\
& \vdots \\
\dot{x}_{n} & =\dot{z}_{n}
\end{array}\right.\right.
$$

O jacobiano da transformação acima tem módulo unitário, portanto:

$$
\begin{aligned}
& p_{\dot{z}_{1}, \ldots, \dot{z}_{n}}\left(\dot{\beta}, \dot{\alpha}_{2}, \ldots, \dot{\alpha}_{n}\right)= \\
& \quad \frac{p_{\dot{x}_{1}, \ldots, \dot{x}_{n}}\left(\dot{\alpha}_{1}, \ldots, \alpha_{i-1}, \beta \ominus \dot{\alpha}_{1} \ominus \ldots \ominus \dot{\alpha}_{i-1} \ominus \dot{\alpha}_{i+1} \ominus \ldots \ominus \dot{\alpha}_{n}, \dot{\alpha}_{i+1}, \ldots, \dot{\alpha}_{n}\right)}{1}
\end{aligned}
$$

Integrando nas variáveis $\dot{\alpha}_{1}, \ldots, \dot{\alpha}_{i-1}, \dot{\alpha}_{i+1}, \ldots, \dot{\alpha}_{n}$ obtém-se o resultado desejado.

Lema 1.9.7. Seja $\dot{x}$ uma variável aleatória cíclica cuja função densidade de probabilidade seja dada por $p_{\dot{x}}(\dot{\beta})$ e seja $\dot{t}$ uma variável aleatória cíclica tal que $\dot{t}=$ $K \odot \dot{x}, K \in \mathbb{Z}, K \neq 0$. Então, a função densidade de probabilidade de $\dot{t}$ é dada por $p_{\dot{t}}(\dot{\beta})=p_{\dot{x}}\left(\frac{1}{K} \odot \dot{\beta}\right)$. 
Demonstração. Seja $p_{\dot{x}}(\dot{\beta})$ a função distribuição de probabilidade de $\dot{x}$, e sejam $\left\{\dot{x}_{2}\right.$, $\left.\ldots, \dot{x}_{K}\right\}, K-1$ variáveis aleatórias cíclicas tais que $x=\dot{x}_{2}=\ldots=\dot{x}_{K}$. Então, a função distribuição de probabilidade conjunta de $\left\{\dot{x}, \dot{x}_{2}, \ldots, \dot{x}_{K}\right\}$ pode ser escrita como:

$$
p_{\dot{x}, \dot{x}_{2}, \ldots, \dot{x}_{K}}\left(\dot{\alpha_{1}}, \dot{\alpha_{2}}, \ldots, \dot{\alpha_{K}}\right)=p_{\dot{x}}\left(\dot{\alpha_{1}}\right) \delta\left(\dot{\alpha_{2}} \ominus \dot{\alpha_{1}}\right) \ldots \delta\left(\dot{\alpha_{K}} \ominus \dot{\alpha_{1}}\right)
$$

Se $K>0, K \in \mathbb{Z}$ :

$$
\dot{t}=\dot{s}=K \odot \dot{x}=\underbrace{\dot{x} \oplus \cdots \oplus \dot{x}}_{\mathrm{K} \text { vezes }}=\dot{x} \oplus \dot{x_{2}} \oplus \cdots \oplus \dot{x}_{K}
$$

Então, usando o teorema 1.9.6 $\operatorname{com} i=n=K$ :

$$
\begin{aligned}
p_{\dot{t}}(\dot{\beta})= & \int_{\mathbb{G}_{\Delta}} \ldots \int_{\mathbb{G}_{\Delta}} p_{\dot{x}}\left(\dot{\alpha}_{1}\right) \delta\left(\dot{\alpha}_{2} \ominus \dot{\alpha}_{1}\right) \ldots \delta\left(\dot{\alpha}_{\dot{K}-1} \ominus \dot{\alpha}_{1}\right) \\
& \delta\left(\dot{\beta} \ominus \dot{\alpha}_{1} \ominus \ldots \ominus \alpha_{\dot{K}-1} \ominus \dot{\alpha}_{1}\right) d \dot{\alpha}_{1} \ldots d \dot{\alpha}_{K-1} \\
= & \int_{\mathbb{G}_{\Delta}} \ldots \int_{\mathbb{G}_{\Delta}} p_{\dot{x}}\left(\dot{\alpha}_{1}\right) \delta\left(\dot{\alpha_{2}} \ominus \dot{\alpha}_{1}\right) \ldots \delta\left(\alpha_{\dot{K}-1} \ominus \dot{\alpha}_{1}\right) \\
& \delta\left(\dot{\beta} \ominus 2 \odot \dot{\alpha}_{1} \ominus \ldots \ominus \alpha_{\dot{K}-1}\right) d \dot{\alpha}_{1} \ldots d \dot{\alpha}_{K-1} \\
= & \int_{\mathbb{G}_{\Delta}} \ldots \int_{\mathbb{G}_{\Delta}} p_{\dot{x}}\left(\dot{\alpha_{1}}\right) \delta\left(\dot{\alpha}_{2} \ominus \dot{\alpha}_{1}\right) \ldots \delta\left(\alpha_{K-2} \ominus \dot{\alpha_{1}}\right) \\
& \delta\left(\dot{\beta} \ominus 3 \odot \dot{\alpha}_{1} \ominus \ldots \ominus \dot{\alpha}_{\dot{K}-2}\right) d \dot{\alpha}_{1} \ldots d \dot{\alpha}_{K-2} \\
= & \int_{\mathbb{G}_{\Delta}} p_{\dot{x}}\left(\dot{\alpha}_{1}\right) \delta\left(\dot{\beta} \ominus K \odot \dot{\alpha}_{1}\right) d \dot{\alpha}_{1}=p_{\dot{x}}\left(\frac{1}{K} \odot \dot{\beta}\right)
\end{aligned}
$$

Se $K<0, K \in \mathbb{Z}$, então $\dot{t}=-\dot{s}$, e se $\dot{s}$ é uniforme, $\dot{t}$ também o será.

Corolario 1.9.8. Seja $\dot{x}$ uma variável aleatória cíclica uniforme e seja $\dot{t}=K \odot$ $\dot{x}, K \in \mathbb{Z}, K \neq 0$. Então, $\dot{t}$ é uniforme.

Demonstração. Pelo lema 1.9.7:

$$
p_{\dot{t}}(\dot{\alpha})=p_{K \odot \dot{x}}(\dot{\alpha})=p_{\dot{x}}\left(\frac{1}{K} \odot \dot{\alpha}\right)=\frac{1}{\Delta} .
$$


Corolario 1.9.9. Nas mesmas condições dos corolários anteriores, as variáveis aleatórias cíclicas $\dot{z}$ e $\dot{w}$ são independentes.

Demonstração.

$$
\left\{\begin{array}{l}
\dot{z}=\dot{x} \oplus \dot{y} \\
\dot{w}=\dot{x} \ominus \dot{y}
\end{array} \quad \Rightarrow \quad \dot{z}=2 \odot \dot{x} \ominus \dot{w}\right.
$$

Pelo corolário 1.9.8, $2 \odot \dot{x}$ é uniforme, consequentemente, pelo corolário 1.9.5, $\dot{z}$ é independente de $\dot{w}$.

Isto que quer dizer que independente da distribuição de uma das parcelas, sempre que se somar uma variável aleatória cíclica com uma variável aleatória cíclica uniforme, a variável aleatória cíclica resultante também será uniforme. Tem-se ainda os resultados surpreendentes que a soma ou a subtração de duas variáveis aleatórias cíclicas independem de uma das parcelas, quando a outra parcela for uniforme, e ainda quando uma parcela for uniforme, a soma é independente da subtração.

\subsection{O Mapa $\mathbf{T}: \mathbf{M}^{+}\left(\mathbb{R}, \Sigma_{\mathbb{R}}\right) \longrightarrow \mathbf{M}^{+}\left(\mathbb{G}_{\Delta}, \Sigma_{\mathbb{G}_{\Delta}}\right)$}

Considere o mapa:

$$
\begin{aligned}
\mathbf{T}: \mathbf{M}^{+}\left(\mathbb{R}, \Sigma_{\mathbb{R}}\right) & \longrightarrow \mathbf{M}^{+}\left(\mathbb{G}_{\Delta}, \Sigma_{\mathbb{G}_{\Delta}}\right) \\
p_{z}(\alpha) & \longmapsto p_{\dot{z}}(\dot{\alpha})=\sum_{n=-\infty}^{\infty} p_{z}\left(\Pi_{[a, \Delta]}(\dot{\alpha})+n \Delta\right)
\end{aligned}
$$

O mapa $\mathbf{T}$ leva, em particular, a densidade de probabilidade de uma variável vista no conjunto dos reais para a densidade de probabilidade correspondente no conjunto cíclico. É imediato mostrar que o mapa $\mathbf{T}$ é linear e sobrejetor. Porém, também 
é simples mostrar que o mapa T é não-injetor. Na verdade, existe uma infinidade de funções em $\mathbf{M}^{+}\left(\mathbb{R}, \Sigma_{\mathbb{R}}\right)$ que chegam no mesmo elemento de $\mathbf{M}^{+}\left(\mathbb{G}_{\Delta}, \Sigma_{\mathbb{G}_{\Delta}}\right)$. É possível então criar a partir do mapa $\mathbf{T}$ uma nova relação de equivalência, agora em $\mathbf{M}^{+}\left(\mathbb{R}, \Sigma_{\mathbb{R}}\right)$ da forma:

$$
p_{z_{1}}(\alpha)=p_{z_{2}}(\alpha) \Leftrightarrow p_{\dot{z}_{1}}(\alpha)=p_{\dot{z}_{2}}(\alpha)
$$

A interpretação desta relação de equivalência é a seguinte: o mapa T é uma maneira de se transportar todo o conhecimento existente das variáveis aleatórias reais para as variáveis aleatórias cíclicas, porém ele significa que nem tudo que se faz com as variáveis aleatórias reais gera alguma novidade. Este mapa tem sido instintivamente usado pelas pessoas ao se dizer por exemplo que uma determinada variável aleatória representando um ângulo é gaussiana. Ora, como o mapa T é não injetor, existe mais de uma variável aleatória que ao ser mapeada por $\mathbf{T}$ torna-se igual à transformada da gaussiana, por exemplo.

\subsection{Séries de Fourier Cíclicas}

Definição 1.11.1. Função cíclica. Uma função cíclica é qualquer mapa do tipo

$$
\dot{f}: \mathbb{G}_{\Delta} \longrightarrow \mathbb{R}
$$

Por estarem definidas em um domínio limitado, as funções cíclicas podem ser representadas em série de Fourier complexa da seguinte forma:

Definição 1.11.2. Série de Fourier cíclica.

$$
\dot{f}(\dot{x})=\sum_{n=-\infty}^{\infty} c_{n} \exp \left(i \frac{2 \pi n}{\Delta} \Pi_{[a, \Delta]}(\dot{x})\right)
$$




$$
\text { onde } \quad c_{n}=\frac{1}{\Delta} \int_{\mathbb{G}_{\Delta}} \dot{f}(\dot{x}) \exp \left(-i \frac{2 \pi n}{\Delta} \Pi_{[a, \Delta]}(\dot{x})\right) d \dot{x}
$$

Teorema 1.11.3. Convolução de séries de Fourier cíclicas. Sejam $c_{n}$ e $d_{m}$ os coeficientes das séries de Fourier cíclicas das funções $\dot{f}(\dot{x})$ e $\dot{g}(\dot{x})$ respectivamente. Então os coeficientes $b_{n}$ da série de Fourier cíclica da convolução entre $\dot{f}(\dot{x})$ e $\dot{g}(\dot{x})$ são dados por

$$
b_{n}=\Delta c_{n} d_{n}
$$

Demonstração. Note que pela proposição 1.4.17

$$
\begin{aligned}
\Pi_{[a, \Delta]}(\dot{x} \ominus \dot{t}) & =\Pi_{[a, \Delta]}(\dot{x})-\Pi_{[a, \Delta]}(\dot{t})+\left\lceil\frac{\Pi_{[a, \Delta]}(\dot{x})-\Pi_{[a, \Delta]}(\dot{t})}{\Delta}\right\rceil \times \Delta \\
& =\Pi_{[a, \Delta]}(\dot{x})-\Pi_{[a, \Delta]}(\dot{t})+k \Delta, k \in \mathbb{Z}
\end{aligned}
$$

Então,

$$
\begin{aligned}
& \int_{\mathbb{G}_{\Delta}} \dot{f}(\dot{t}) \dot{g}(\dot{x} \ominus \dot{t}) d \dot{t} \\
= & \int_{\mathbb{G}_{\Delta}}\left[\sum_{n=-\infty}^{\infty} c_{n} \exp \left(i \frac{2 \pi n}{\Delta} \Pi_{[a, \Delta]}(\dot{t})\right)\right]\left[\sum_{m=-\infty}^{\infty} d_{m} \exp \left(i \frac{2 \pi m}{\Delta} \Pi_{[a, \Delta]}(\dot{x} \ominus \dot{t})\right)\right] d \dot{t} \\
= & \sum_{n=-\infty}^{\infty} \sum_{m=-\infty}^{\infty} c_{n} d_{m} \int_{\mathbb{G}_{\Delta}} \exp \left[i \frac{2 \pi}{\Delta}\left(n \Pi_{[a, \Delta]}(\dot{t})+m\left(\Pi_{[a, \Delta]}(\dot{x})-\Pi_{[a, \Delta]}(\dot{t})+k \Delta\right)\right)\right] d \dot{t} \\
= & \sum_{n=-\infty}^{\infty} \sum_{m=-\infty}^{\infty} c_{n} d_{m} \exp \left(i \frac{2 \pi m}{\Delta} \Pi_{[a, \Delta]}(\dot{x})\right) \int_{\mathbb{G}_{\Delta}} \exp \left(i \frac{2 \pi(n-m)}{\Delta} \Pi_{[a, \Delta]}(\dot{t})\right) d \dot{t}
\end{aligned}
$$

(Teorema 1.8.7)

$$
\begin{aligned}
& =\sum_{n=-\infty}^{\infty} \sum_{m=-\infty}^{\infty} c_{n} d_{m} \exp \left(i \frac{2 \pi m}{\Delta} \Pi_{[a, \Delta]}(\dot{x})\right) \int_{I_{[a, \Delta]}} \exp \left(i \frac{2 \pi(n-m)}{\Delta} t\right) d t \\
& =\sum_{n=-\infty}^{\infty} \sum_{m=-\infty}^{\infty} c_{n} d_{m} \exp \left(i \frac{2 \pi m}{\Delta} \Pi_{[a, \Delta]}(\dot{x})\right) \Delta \delta_{n m} \\
& =\sum_{n=-\infty}^{\infty} \Delta c_{n} d_{n} \exp \left(i \frac{2 \pi n}{\Delta} \Pi_{[a, \Delta]}(\dot{x})\right)=\sum_{n=-\infty}^{\infty} b_{n} \exp \left(i \frac{2 \pi n}{\Delta} \Pi_{[a, \Delta]}(\dot{x})\right)
\end{aligned}
$$


A seguir, alguns resultados úteis que podem ser usados mais adiante.

Proposição 1.11.4. Os coeficientes da série de Fourier do impulso centrado em $x=\mu$ são dados por

$$
c_{n}=\frac{1}{\Delta} \exp \left(-i \frac{2 \pi n}{\Delta} \mu\right)
$$

Demonstração.

$$
\begin{aligned}
c_{n}= & \frac{1}{\Delta} \int_{\mathbb{G}_{\Delta}} \delta(\dot{x} \ominus \dot{\mu}) \exp \left(-i \frac{2 \pi n}{\Delta} \Pi_{[a, \Delta]}(\dot{x})\right) d \dot{x} \\
& \frac{1}{\Delta} \exp \left(-i \frac{2 \pi n}{\Delta} \Pi_{[a, \Delta]}(\dot{\mu})\right) \\
& \frac{1}{\Delta} \exp \left(-i \frac{2 \pi n}{\Delta} \mu\right)
\end{aligned}
$$

Proposição 1.11.5. Os coeficientes da série de Fourier da distribuição uniforme cíclica são dados por

$$
\left\{\begin{array}{l}
n=0, \quad \Rightarrow c_{0}=\frac{1}{\Delta} \\
n \neq 0, \quad \Rightarrow c_{n}=0
\end{array}\right.
$$

Demonstração.

$$
\begin{aligned}
n=0 & \\
c_{0} & =\frac{1}{\Delta^{2}} \int_{\mathbb{G}_{\Delta}} d \dot{x} \\
& =\frac{1}{\Delta} \\
n \neq 0 & \\
c_{n} & =\frac{1}{\Delta^{2}} \int_{\mathbb{G}_{\Delta}} \exp \left(-i \frac{2 \pi n}{\Delta} \Pi_{[a, \Delta]}(\dot{x})\right) d \dot{x}
\end{aligned}
$$


$($ Ortogonalidade $)=0$

\subsection{Gaussianas Cíclicas}

O seguinte resultado é conhecido da teoria das variáveis aleatórias reais.

Teorema 1.12.1. A soma de duas variáveis aleatórias gaussianas reais e independentes é uma variável aleatória gaussiana real com média igual à soma das médias das parcelas e variância igual à soma das variâncias das parcelas.

O teorema 1.12.1 decorre diretamente do fato de que a convolução de duas gaussianas é uma gaussiana com as propriedades descritas acima. Na teoria das variáveis aleatórias cíclicas é possível definir um tipo de variável aleatória que possui propriedades semelhantes à da variável aleatória real Gaussiana.

Definição 1.12.2. A Função Gaussiana cíclica é dada por

$$
\begin{aligned}
\dot{N}\left(\mu, \sigma^{2}\right): & \mathbb{G}_{\Delta} \longrightarrow \mathbb{R} \\
& \dot{x} \longmapsto \dot{N}\left(\dot{x}, \mu, \sigma^{2}\right)=\sum_{n=-\infty}^{\infty} \frac{1}{\sqrt{2 \pi} \sigma} \exp \left(-\frac{\left(\Pi_{[a, \Delta]}(\dot{x})-\mu+n \Delta\right)^{2}}{2 \sigma^{2}}\right)
\end{aligned}
$$

Teorema 1.12.3. Os coeficientes da série de Fourier cíclica da função Gaussiana cíclica $\dot{N}\left(\mu, \sigma^{2}\right)$ são dados por

$$
c_{n}=\frac{1}{\Delta} \exp \left[-2 \sigma^{2}\left(\frac{\pi n}{\Delta}\right)\left(\frac{\pi n}{\Delta}+i \frac{\mu}{\sigma^{2}}\right)\right]
$$

Demonstração.

$$
c_{n}=\frac{1}{\Delta} \int_{\mathbb{G}_{\Delta}} \dot{N}\left(\dot{x}, \mu, \sigma^{2}\right) \exp \left(-i \frac{2 \pi n}{\Delta} \Pi_{[a, \Delta]}(\dot{x})\right) d \dot{x}
$$


$=\frac{1}{\Delta} \int_{\mathbb{G}_{\Delta}} \sum_{l=-\infty}^{\infty} \frac{1}{\sqrt{2 \pi} \sigma} \exp \left[-\frac{\left(\Pi_{[a, \Delta]}(\dot{x})-\mu+l \Delta\right)^{2}}{2 \sigma^{2}}\right] \exp \left(-i \frac{2 \pi n}{\Delta} \Pi_{[a, \Delta]}(\dot{x})\right) d \dot{x}$ (Teorema 1.8.7)

$$
\begin{aligned}
= & \frac{1}{\Delta} \int_{I_{[a, \Delta]}} \sum_{l=-\infty}^{\infty} \frac{1}{\sqrt{2 \pi} \sigma} \exp \left[-\frac{(x-\mu+l \Delta)^{2}}{2 \sigma^{2}}\right] \exp \left(-i \frac{2 \pi n}{\Delta} x\right) d x \\
& (y=x+n \Delta) \\
= & \frac{1}{\sqrt{2 \pi} \sigma \Delta} \sum_{l=-\infty}^{\infty} \int_{a+l \Delta}^{a+(l+1) \Delta} \exp \left[-\frac{(y-\mu)^{2}}{2 \sigma^{2}}-i \frac{2 \pi n y}{\Delta}+i \frac{2 \pi n l}{\Delta}\right] d y \\
= & \frac{1}{\sqrt{2 \pi} \sigma \Delta} \int_{-\infty}^{\infty} \exp \left[-\frac{(y-\mu)^{2}}{2 \sigma^{2}}-i \frac{2 \pi n}{\Delta} y\right] d y
\end{aligned}
$$

Mas

$$
\begin{aligned}
& -\frac{(y-\mu)^{2}}{2 \sigma^{2}}-i \frac{2 \pi n y}{\Delta} \\
= & -\frac{1}{2 \sigma^{2}}\left[y^{2}-2 \mu y+\mu^{2}+i \frac{4 \sigma^{2} \pi n y}{\Delta}\right] \\
= & -\frac{1}{2 \sigma^{2}}\left[y^{2}-2\left(\mu-i \frac{2 \sigma^{2} \pi n}{\Delta}\right) y+\mu^{2}\right] \\
= & -\frac{1}{2 \sigma^{2}}\left[\left(y-\mu+i \frac{2 \sigma^{2} \pi n}{\Delta}\right)^{2}+\mu^{2}-\mu^{2}+i \frac{4 \sigma^{2} \pi n \mu}{\Delta}+\frac{4 \sigma^{4} \pi^{2} n^{2}}{\Delta^{2}}\right] \\
= & -\frac{1}{2 \sigma^{2}}\left[\left(y-\mu+i \frac{2 \sigma^{2} \pi n}{\Delta}\right)^{2}+4 \sigma^{4}\left(\frac{\pi n}{\Delta}\right)\left(\frac{\pi n}{\Delta}+i \frac{\mu}{\sigma^{2}}\right)\right] \\
= & -\frac{\left(y-\mu+i \frac{2 \sigma^{2} \pi n}{\Delta}\right)^{2}}{2 \sigma^{2}}-2 \sigma^{2}\left(\frac{\pi n}{\Delta}\right)\left(\frac{\pi n}{\Delta}+i \frac{\mu}{\sigma^{2}}\right)
\end{aligned}
$$

Portanto

$$
\begin{aligned}
c_{n} & =\frac{1}{\Delta} \exp \left[-2 \sigma^{2}\left(\frac{\pi n}{\Delta}\right)\left(\frac{\pi n}{\Delta}+i \frac{\mu}{\sigma^{2}}\right)\right] \frac{1}{\sqrt{2 \pi} \sigma} \int_{-\infty}^{\infty} \exp \left[-\frac{\left(y-\mu+i \frac{2 \sigma^{2} \pi n}{\Delta}\right)^{2}}{2 \sigma^{2}}\right] d y \\
& =\frac{1}{\Delta} \exp \left[-2 \sigma^{2}\left(\frac{\pi n}{\Delta}\right)\left(\frac{\pi n}{\Delta}+i \frac{\mu}{\sigma^{2}}\right)\right]
\end{aligned}
$$


Teorema 1.12.4. A convolução de duas gaussianas cíclicas é também uma Gaussiana cíclica, onde o parâmetro $\mu$ é a soma dos parâmetros $\mu$ e o parâmetro $\sigma^{2}$ é a soma dos parâmetros $\sigma^{2}$ das gaussianas cíclicas originais, isto é:

$$
\dot{N}\left(\mu_{1}, \sigma_{1}^{2}\right) \otimes \dot{N}\left(\mu_{2}, \sigma_{2}^{2}\right)=\dot{N}\left(\mu_{1}+\mu_{2}, \sigma_{1}^{2}+\sigma_{2}^{2}\right)
$$

Demonstração. Pelo teorema 1.12.3, tem-se que

$$
\begin{aligned}
c_{n} & =\frac{1}{\Delta} \exp \left[-2 \sigma_{1}^{2}\left(\frac{\pi n}{\Delta}\right)\left(\frac{\pi n}{\Delta}+i \frac{\mu_{1}}{\sigma_{1}^{2}}\right)\right] \\
\text { e } \quad d_{n} & =\frac{1}{\Delta} \exp \left[-2 \sigma_{2}^{2}\left(\frac{\pi n}{\Delta}\right)\left(\frac{\pi n}{\Delta}+i \frac{\mu_{2}}{\sigma_{2}^{2}}\right)\right]
\end{aligned}
$$

são os coeficientes da série de Fourier cíclica das gaussianas cíclicas $\dot{N}\left(x, \mu_{1}, \sigma_{1}^{2}\right)$ e $\dot{N}\left(x, \mu_{2}, \sigma_{2}^{2}\right)$ respectivamente. Sejam $b_{n}$ os coeficiente da série de Fourier cíclica da convolução entre as duas gaussianas cíclicas anteriores. Usando o teorema 1.11.3, pode-se então escrever

$$
\begin{aligned}
b_{n} & =\Delta c_{n} d_{n} \\
& =\Delta \frac{1}{\Delta} \exp \left[-2 \sigma_{1}^{2}\left(\frac{\pi n}{\Delta}\right)\left(\frac{\pi n}{\Delta}+i \frac{\mu_{1}}{\sigma_{1}^{2}}\right)\right] \frac{1}{\Delta} \exp \left[-2 \sigma_{2}^{2}\left(\frac{\pi n}{\Delta}\right)\left(\frac{\pi n}{\Delta}+i \frac{\mu_{2}}{\sigma_{2}^{2}}\right)\right] \\
& =\frac{1}{\Delta} \exp \left[-\left(\frac{2 \pi n}{\Delta}\right)\left(\frac{\pi n \sigma_{1}^{2}}{\Delta}+i \mu_{1}+\frac{\pi n \sigma_{2}^{2}}{\Delta}+i \mu_{2}\right)\right] \\
& =\frac{1}{\Delta} \exp \left\{-\left(\frac{2 \pi n}{\Delta}\right)\left[\frac{\pi n\left(\sigma_{1}^{2}+\sigma_{2}^{2}\right)}{\Delta}+i\left(\mu_{1}+\mu_{2}\right)\right]\right\} \\
& =\frac{1}{\Delta} \exp \left\{-2\left(\sigma_{1}^{2}+\sigma_{2}^{2}\right)\left(\frac{\pi n}{\Delta}\right)\left[\frac{\pi n}{\Delta}+i\left(\frac{\mu_{1}+\mu_{2}}{\sigma_{1}^{2}+\sigma_{2}^{2}}\right)\right]\right\}
\end{aligned}
$$

Usando mais uma vez o teorema 1.12.3, é possível concluir o resultado desejado.

\section{Proposição 1.12.5.}

$$
\lim _{\sigma \rightarrow \infty} \dot{N}\left(\dot{x}, \mu, \sigma^{2}\right) \equiv \frac{1}{\Delta}
$$


Demonstração.

$$
\begin{aligned}
\lim _{\sigma \rightarrow \infty} \dot{N}\left(\dot{x}, \mu, \sigma^{2}\right) & =\lim _{\sigma \rightarrow \infty} \sum_{n=-\infty}^{\infty} c_{n} \exp \left(i \frac{2 \pi n}{\Delta} \Pi_{[a, \Delta]}(\dot{x})\right) \\
\text { (série converge } & =c_{0}+\sum_{n=-\infty, n \neq 0}^{\infty} \lim _{\sigma \rightarrow \infty}\left[c_{n} \exp \left(i \frac{2 \pi n}{\Delta} \Pi_{[a, \Delta]}(\dot{x})\right)\right] \\
\text { ponto a ponto) } & =\frac{1}{\Delta}+\sum_{n=-\infty, n \neq 0}^{\infty}\left[\exp \left(i \frac{2 \pi n}{\Delta} \Pi_{[a, \Delta]}(\dot{x})\right)\right. \\
& \left.=\frac{1}{\Delta}+\sum_{\sigma \rightarrow \infty}^{\infty} \frac{1}{\Delta} \exp \left[-2 \sigma^{2}\left(\frac{\pi n}{\Delta}\right)\left(\frac{\pi n}{\Delta}+i \frac{\mu}{\sigma^{2}}\right)\right]\right] \\
& =\frac{1}{\Delta}
\end{aligned}
$$




\section{Capítulo 2}

\section{Modelos Gaussianos de Ruído Interferométrico}

\subsection{Introdução}

Neste capítulo será introduzido e discutido o ruído interferométrico. Serão estudados dois modelos gaussianos para ruído interferométrico que são bastante utilizados na literatura técnica. Será ainda realizada uma análise detalhada das hipóteses que estes modelos pressupõem, além de uma avaliação da validade das mesmas de um ponto de vista prático.

\subsection{Ruído Interferométrico}

Dá-se o nome de ruído interferométrico ao fenômeno de interferência que ocorre no interior das fibras óticas, devido à reflexões nas descontinuidades do meio ( e.g. conectores e emendas ). Essas reflexões fazem com que o sinal interfira com versões anteriores dele mesmo ( crosstalks ). O desenvolvimento dos amplificadores óticos possibilitou a construção de redes totalmente óticas, onde o sinal ótico percorre 
longas distâncias sem a necessidade de regeneradores. Porém, com a eliminação dos regeneradores, o ruído interferométrico que antes era eliminado nestes estágios passou a se acumular na rede, tornando-se fator limitante no projeto destas.

\subsection{Expressão usual da literatura}

Originalmente encontrada em [14], a expressão (2.3.1) tenta modelar a penalidade de potência de um sistema com ruído interferométrico para o caso de um único sinal de crosstalk, supondo uma distribuição gaussiana para a intensidade do sinal detectado no sensor. A penalidade de potência é a quantidade de potência que deve ser dada a mais num sistema para que ele tenha um desempenho, medido em termos da probabilidade de erro, igual ao sistema sem ruído interferométrico.

$$
P P=-5 \log \left(1-4 q^{2} \mathbf{E}\left[R^{2}\right]\right)
$$

Nesta expressão, $q$ é uma constante tal que $\frac{1}{2} Q(q)=10^{-9}$ e $R=\beta \sqrt{R_{1} R_{2}} \vec{p}(t) \cdot \vec{p}(t-\tau)$, onde $\beta$ é a atenuação sofrida pelo sinal de crosstalk devido a perda na fibra, $R_{1}$ e $R_{2}$ são os coeficientes de reflexão das descontinuidades do meio onde a luz se propaga e $\vec{p}$ é o vetor de polarização da luz.

A seguir será desenvolvida a argumentação que leva a esse resultado, a fim de que as hipóteses assumidas fiquem claras. Seja $\vec{E}_{0}(t)=b_{0}(t) E_{0} e^{j(\omega t+\phi(t))} \vec{p}_{0}(t)$ a expressão do campo elétrico, onde $E_{0}=\sqrt{2 P_{s}}$ e $P_{s}$ é o valor RMS da potência ótica no detector, $b_{0}(t)$ é o bit de informação no instante $t$, que pode ser 0 ou 1, $\omega$ é a freqüência ótica, $\phi(t)$ é o ruído de fase e $p(\vec{t})$ é o vetor de polarização. Pode-se então escrever o campo total como

$$
\vec{E}_{T}(t)=\vec{E}_{0}(t)+\sum_{i=1}^{N} \sqrt{\alpha_{i}} \vec{E}_{0}\left(t-\tau_{i}\right)=
$$




$$
E_{0}\left[b_{0}(t) e^{j(\omega t+\phi(t))} \vec{p}_{0}(t)+\sum_{i=1}^{N} b_{0}\left(t-\tau_{i}\right) \sqrt{\alpha_{i}} e^{j\left(\omega\left(t-\tau_{i}\right)+\phi\left(t-\tau_{i}\right)\right)} \vec{p}_{0}\left(t-\tau_{i}\right)\right],
$$

Onde $\alpha_{i}$ representa a atenuação total sofrida pelo $i$-ésimo sinal de crosstalk. Ou ainda, fazendo $b_{0}\left(t-\tau_{i}\right)=b_{i}(t)$ e $\vec{p}_{0}\left(t-\tau_{i}\right)=\vec{p}_{i}(t)$

$$
\vec{E}_{T}(t)=E_{0}\left[b_{0}(t) e^{j(\omega t+\phi(t))} \vec{p}_{0}(t)+\sum_{i=1}^{N} b_{i}(t) \sqrt{\alpha_{i}} e^{j\left(\omega\left(t-\tau_{i}\right)+\phi\left(t-\tau_{i}\right)\right)} \vec{p}_{i}(t)\right]
$$

Desprezando os termos de crosstalk secundários, a intensidade da luz vale

$$
\begin{aligned}
I(t)= & \left\|\vec{E}_{T}(t)\right\|^{2}=E_{0}^{2}\left[b_{0}^{2}(t)+\sum_{i=1}^{N} b_{i}^{2}(t) \alpha_{i}+\right. \\
& \left.2 b_{0}(t) \sum_{i=1}^{N} b_{i}(t) \sqrt{\alpha_{i}} \cos \left(\omega_{0} \tau_{i}+\phi(t)-\phi\left(t-\tau_{i}\right)\right) \cos \beta_{0 i}\right]+n_{T}(t),
\end{aligned}
$$

onde $\vec{p}_{i}(t) \cdot \vec{p}_{k}(t)=\cos \beta_{i k}$ e $n_{T}(t)$ é o ruído térmico. Então, usando a relação $b_{i}^{2}(t)=$ $b_{i}(t)$, tem-se

$$
\begin{aligned}
I(t)= & E_{0}^{2}\left[b_{0}(t)+\sum_{i=1}^{N} b_{i}(t)\left\{\alpha_{i}+\right.\right. \\
& \left.\left.2 b_{0}(t) \sqrt{\alpha_{i}} \cos \left(\omega_{0} \tau_{i}+\phi(t)-\phi\left(t-\tau_{i}\right)\right) \cos \beta_{0 i}\right\}\right]+n_{T}(t) .
\end{aligned}
$$

Esquecendo a dependência em t e fazendo $X_{i 0}=\cos \left(\omega_{0} \tau_{i}+\phi(t)-\phi\left(t-\tau_{i}\right)\right)$ e $Y_{i 0}=\cos \beta_{i 0}$

$$
I=E_{0}^{2}\left[b_{0}+\sum_{i=1}^{N} b_{i}\left\{\alpha_{i}+2 b_{0} \sqrt{\alpha_{i}} X_{i 0} Y_{i 0}\right\}\right]+n_{T}
$$

Seja $D$ o nível de decisão do sistema. Isto significa que o bit será considerado 0 se o nível de tensão estiver abaixo de $D$, e 1 caso contrário. Então a probabilidade de erro pode ser escrita como

$$
P_{e}=\mathbf{P}\left[I>D \mid b_{0}=0\right] \cdot \mathbf{P}\left[b_{0}=0\right]+\mathbf{P}\left[I<D \mid b_{0}=1\right] \cdot \mathbf{P}\left[b_{0}=1\right]
$$


Para calcular a expressão acima, é necessário calcular alguns parâmetros associados às seguintes variáveis aleatórias condicionadas

$$
\begin{aligned}
& {\left[I \mid b_{0}=0\right]=E_{0}^{2} \sum_{i=1}^{N} b_{i} \alpha_{i}+n_{T}} \\
& {\left[I \mid b_{0}=1\right]=E_{0}^{2}\left[1+\sum_{i=1}^{N} b_{i}\left\{\alpha_{i}+2 \sqrt{\alpha_{i}} X_{i 0} Y_{i 0}\right\}\right]+n_{T} .}
\end{aligned}
$$

A partir destas expressões, é possível calcular as médias e as variâncias das variáveis condicionadas

$$
\begin{aligned}
\mu_{0_{N}} & =\mathbf{E}\left[I \mid b_{0}=0\right]=E_{0}^{2} \sum_{i=1}^{N} \alpha_{i} \mathbf{E}\left[b_{i}\right]+\mathbf{E}\left[n_{T}\right]=\frac{E_{0}^{2}}{2} \sum_{i=1}^{N} \alpha_{i} \\
\mu_{1_{N}} & =\mathbf{E}\left[I \mid b_{0}=1\right]=E_{0}^{2}\left[1+\sum_{i=1}^{N}\left\{\alpha_{i} \mathbf{E}\left[b_{i}\right]+2 \sqrt{\alpha_{i}} \mathbf{E}\left[b_{i} X_{i 0} Y_{i 0}\right]\right\}+\mathbf{E}\left[n_{T}\right]\right. \\
& =E_{0}^{2}\left(1+\sum_{i=1}^{N} \frac{\alpha_{i}}{2}\right) \\
\sigma_{0_{N}}^{2} & =\mathbf{V}\left[I \mid b_{0}=0\right]=E_{0}^{4} \sum_{i=1}^{N} \alpha_{i}^{2} \mathbf{V}\left[b_{i}\right]+\mathbf{V}\left[n_{T}\right]=\frac{E_{0}^{4}}{4} \sum_{i=1}^{N} \alpha_{i}^{2}+\sigma_{T}^{2} \\
\sigma_{1_{N}}^{2} & =\mathbf{V}\left[I \mid b_{0}=1\right]=E_{0}^{4} \sum_{i=1}^{N}\left\{\alpha_{i}^{2} \mathbf{V}\left[b_{i}\right]+4 \alpha_{i} \mathbf{V}\left[b_{i} X_{i 0} Y_{i 0}\right]\right\}+\mathbf{V}\left[n_{T}\right] \\
& =\frac{E_{0}^{4}}{4} \sum_{i=1}^{N}\left\{\alpha_{i}^{2}+4 \alpha_{i} \mathbf{V}\left[X_{i 0} Y_{i 0}\right]\right\}+\sigma_{T}^{2}
\end{aligned}
$$

O que implica em

$$
\begin{aligned}
\mu_{1_{N}} & =\mu_{0_{N}}+E_{0}^{2} \\
\sigma_{1_{N}}^{2} & =\sigma_{0_{N}}^{2}+E_{0}^{4} \sum_{i=1}^{N} \alpha_{i} \mathbf{V}\left[X_{i 0} Y_{i 0}\right] \\
& \Rightarrow\left(\sigma_{1_{N}}^{2}-\sigma_{0_{N}}^{2}\right)=\left(\mu_{1_{N}}-\mu_{0_{N}}\right)^{2} \sum_{i=1}^{N} \alpha_{i} \mathbf{V}\left[X_{i 0} Y_{i 0}\right] .
\end{aligned}
$$

Note que quando $N=0$, isto é, nenhum crosstalk, tem-se

$$
\mu_{0_{0}}=0
$$




$$
\begin{aligned}
& \mu_{1_{0}}=E_{0}^{2} \\
& \sigma_{0_{0}}^{2}=\sigma_{T}^{2} \\
& \sigma_{1_{0}}^{2}=\sigma_{T}^{2} .
\end{aligned}
$$

Agora é possível calcular a probabilidade de erro. Lembrando que

$$
Q(x)=\frac{1}{\sqrt{2 \pi}} \int_{x}^{\infty} e^{\frac{-u^{2}}{2}} d u
$$

se as variáveis $\left[I \mid b_{0}=0\right]$ e $\left[I \mid b_{0}=1\right]$ forem aproximadas por variáveis gaussianas, pode-se escrever

$$
\begin{aligned}
P_{e_{N}}= & \frac{1}{2}\left(\mathbf{P}\left[I>D \mid b_{0}=0\right]+\mathbf{P}\left[I<D \mid b_{0}=1\right]\right) \\
= & \frac{1}{2}\left(\mathbf{P}\left[\frac{I-\mu_{0_{N}}}{\sigma_{0_{N}}}>\frac{D-\mu_{0_{N}}}{\sigma_{0_{N}}} \mid b_{0}=0\right]\right. \\
& \left.+\mathbf{P}\left[\frac{I-\mu_{1_{N}}}{\sigma_{1_{N}}}<\frac{D-\mu_{1_{N}}}{\sigma_{1_{N}}} \mid b_{0}=1\right]\right) \\
= & \frac{1}{2}\left[Q\left(\frac{D-\mu_{0_{N}}}{\sigma_{0_{N}}}\right)+Q\left(\frac{\mu_{1_{N}}-D}{\sigma_{1_{N}}}\right)\right] .
\end{aligned}
$$

Se o nível de decisão utilizado for

$$
D_{N}=\frac{\mu_{0_{N}}+\mu_{1_{N}}}{2}
$$

a expressão da probabilidade de erro é dada por

$$
P_{e_{N}}=\frac{1}{2}\left[Q\left(\frac{\mu_{1_{N}}-\mu_{0_{N}}}{2 \sigma_{0_{N}}}\right)+Q\left(\frac{\mu_{1_{N}}-\mu_{0_{N}}}{2 \sigma_{1_{N}}}\right)\right]
$$

Definindo $\xi_{N}=\frac{\mu_{1_{N}}-\mu_{0_{N}}}{\sigma_{0}}$ e substituindo a equação (2.3.15) na equação (2.3.22), obtém-se

$$
P_{e}^{s i m p l}\left(\xi_{N}\right)=\frac{1}{2}\left[Q\left(\frac{\xi_{N}}{2}\right)+Q\left(\frac{\xi_{N}}{2} \frac{1}{\sqrt{1+\xi_{N}^{2} \sum_{i=1}^{N} \alpha_{i} \mathbf{V}\left[X_{i 0} Y_{i 0}\right]}}\right)\right] .
$$


A notação $P_{e}^{s i m p l}$ quer dizer que este é o modelo gaussiano "simplificado", em oposição ao modelo "completo" que será visto mais adiante.

A segunda parcela da expressão (2.3.23) é sempre maior do que a primeira pois o argumento da função Q é sempre menor na segunda parcela. Por ora, suponha que a segunda parcela seja muito maior que a primeira de modo que seja possível escrever

$$
P_{e_{N}}=\frac{1}{2} Q\left(\frac{\xi_{N}}{2} \frac{1}{\sqrt{1+\xi_{N}^{2} \sum_{i=1}^{N} \alpha_{i} \mathbf{V}\left[X_{i 0} Y_{i 0}\right]}}\right) .
$$

Defina a penalidade de potência como

$$
P P_{N}=10 \log \left(\frac{\left(\mu_{1_{N}}-\mu_{0_{N}}\right)}{\left(\mu_{1_{0}}-\mu_{0_{0}}\right)}\right) .
$$

Seja q tal que $\frac{1}{2} Q(q)=10^{-9}$. Então para esta taxa de erro

$$
q=\left(\frac{\mu_{1_{N}}-\mu_{0_{N}}}{2 \sigma_{1_{N}}}\right)=\left(\frac{\mu_{1_{0}}-\mu_{0_{0}}}{2 \sigma_{1_{0}}}\right)
$$

o que quer dizer que

$$
\frac{\left(\mu_{1_{N}}-\mu_{0_{N}}\right)}{\left(\mu_{1_{0}}-\mu_{0_{0}}\right)}=\frac{\sigma_{1_{N}}}{\sigma_{1_{0}}} .
$$

Então

$$
\begin{aligned}
P P_{N} & =10 \log \left(\frac{\left(\mu_{1_{N}}-\mu_{0_{N}}\right)}{\left(\mu_{1_{0}}-\mu_{0_{0}}\right)}\right) \\
& =5 \log \left(\frac{\left(\mu_{1_{N}}-\mu_{0_{N}}\right)^{2}}{\left(\mu_{1_{0}}-\mu_{0_{0}}\right)^{2}}\right) \\
& =5 \log \left(\frac{\sigma_{1_{N}}^{2}}{\sigma_{1_{0}}^{2}}\right)=-5 \log \left(\frac{\sigma_{1_{0}}^{2}}{\sigma_{1_{N}}^{2}}\right) .
\end{aligned}
$$

Quando $N=0$, tem-se $\sigma_{1_{0}}^{2}=\sigma_{0_{0}}^{2}=\sigma_{T}^{2}$. Quando $N>0$, da expressão (2.3.13) tem-se

$$
\sigma_{T}^{2}=\sigma_{1_{N}}^{2}-\frac{\left(\mu_{1}-\mu_{0}\right)_{N}^{2}}{4} \sum_{i=1}^{N}\left\{4 \alpha_{i} \mathbf{V}\left[X_{i 0} Y_{i 0}\right]+\alpha_{i}^{2}\right\} .
$$


Deste modo, substituindo (2.3.29) em (2.3.28), obtém-se a penalidade

$$
\begin{aligned}
P P_{N}= & -5 \log \left[1-\frac{\left(\mu_{1_{N}}-\mu_{0_{N}}\right)^{2}}{4 \sigma_{1_{N}}^{2}} \sum_{i=1}^{N}\left\{4 \alpha_{i} \mathbf{V}\left[X_{i 0} Y_{i 0}\right]+\alpha_{i}^{2}\right\}\right]= \\
& -5 \log \left[1-q^{2} \sum_{i=1}^{N}\left\{4 \alpha_{i} \mathbf{V}\left[X_{i 0} Y_{i 0}\right]+\alpha_{i}^{2}\right\}\right] .
\end{aligned}
$$

A expressão (2.3.1) pode ser obtida da expressão (2.3.30) se o termo em $\alpha^{2}$ for desprezado. Substituindo os valores de variância de X e Y ( vide apêndice I ) tem-se para polarizações alinhadas

$$
P P_{N}=-5 \log \left[1-q^{2} \sum_{i=1}^{N} \alpha_{i}\left(2+\alpha_{i}\right)\right]
$$

e para polarizações aleatórias uniformes

$$
P P_{N}=-5 \log \left[1-q^{2} \sum_{i=1}^{N} \alpha_{i}\left(1+\alpha_{i}\right)\right] .
$$

Se $\alpha_{i}=\alpha, \forall i$, então para polarizações alinhadas

$$
P P_{N}=-5 \log \left[1-q^{2} N \alpha(2+\alpha)\right]
$$

e para polarizações aleatórias uniformes

$$
P P_{N}=-5 \log \left[1-q^{2} N \alpha(1+\alpha)\right]
$$

Vale a pena acrescentar aqui que a suposição que tornou possível simplificar a expressão (2.3.23) e obter a expressão (2.3.24) normalmente só vale para valores de relação sinal ruído elétrica acima de $20 \mathrm{~dB}$, dependendo do valor de $\alpha$, conforme pode ser observado na figura 2.1. Na prática, esta é a faixa de valores em que se trabalha, e todas as medidas realizadas posteriormente neste trabalho estão dentro deste limite, portanto a aproximação é boa. 


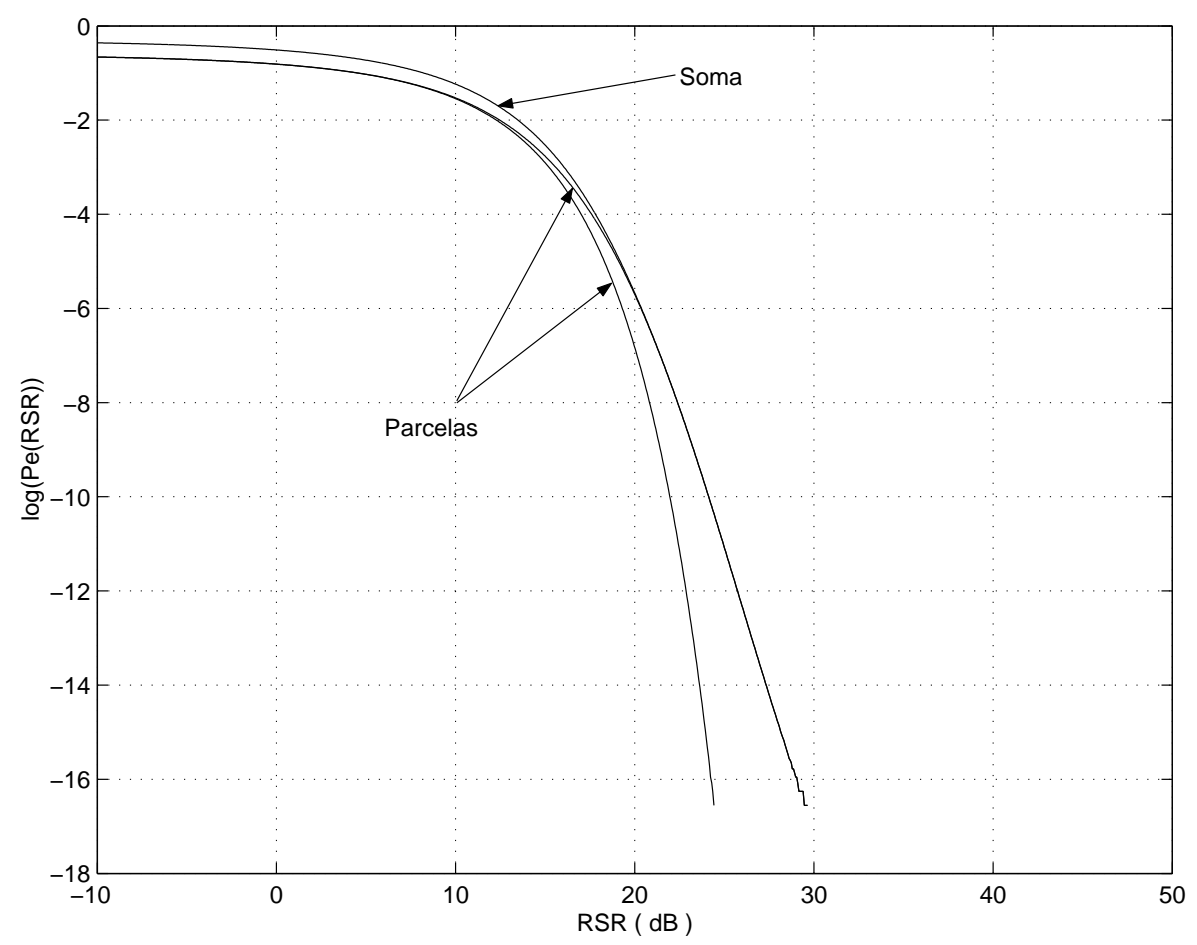

Figura 2.1: Comparação entre as duas parcelas e a soma da expressão (2.3.23)

\subsection{Uma aproximação gaussiana mais realista}

Conforme explicado anteriormente, as expressões obtidas acima supõem que as variáveis $\left[I \mid b_{0}=0\right]$ e $\left[I \mid b_{0}=1\right]$ são Gaussianas. No caso de $\left[I \mid b_{0}=1\right]$, essa é uma suposição forte, uma vez que esta variável se compõe de $N+2$ outras variáveis aleatórias ( $N+1$ se as polarizações estiverem alinhadas ), que já são sabidas de antemão não serem Gaussianas. A fim de se obter uma expressão mais realista para a probabilidade de erro, é possível condicionar a expressão inicial nos valores dos 
bits passados. Então, fazendo $\alpha_{i}=\alpha \forall i$ desde o começo do desenvolvimento das expressões, a probabilidade de erro deixa de depender dos bits individualmente e passa a depender apenas do número de bits não nulos. Seja $n=\sum_{k=1}^{N} b_{k}$. Então

$$
\begin{aligned}
P_{e_{N}}^{c o m p l} & =\mathbf{P}\left[I>D \mid b_{0}=0\right] \cdot \mathbf{P}\left[b_{0}=0\right]+\mathbf{P}\left[I<D \mid b_{0}=1\right] \cdot \mathbf{P}\left[b_{0}=1\right] \\
& =\frac{1}{2}\left(\mathbf{P}\left[I>D \mid b_{0}=0\right]+\mathbf{P}\left[I<D \mid b_{0}=1\right]\right) \\
& =\frac{1}{2} \sum_{i=0}^{N}\left(\mathbf{P}\left[I>D \mid b_{0}=0, n=i\right]+\mathbf{P}\left[I<D \mid b_{0}=1, n=i\right]\right) \mathbf{P}[n=i] \\
& =2^{-(N+1)} \sum_{i=0}^{N}\left(\begin{array}{c}
N \\
i
\end{array}\right)\left(\mathbf{P}\left[I>D \mid b_{0}=0, n=i\right]+\mathbf{P}\left[I<D \mid b_{0}=1, n=i\right]\right) .
\end{aligned}
$$

Mas

$$
\begin{aligned}
\mathbf{P}\left[I>D \mid b_{0}=0, n=i\right] & =\mathbf{P}\left[E_{0}^{2} n \alpha+n_{T}>D \mid b_{0}=0, n=i\right] \\
& =\mathbf{P}\left[\frac{n_{T}}{\sigma_{T}}>\frac{D-E_{0}^{2} n \alpha}{\sigma_{T}} \mid b_{0}=0, n=i\right] \\
& =Q\left(\frac{D-E_{0}^{2} i \alpha}{\sigma_{T}}\right)=Q\left(\frac{D-\left(\mu_{1_{i}}-\mu_{0_{i}}\right) i \alpha}{\sigma_{T}}\right)
\end{aligned}
$$

e

$$
\begin{aligned}
\mathbf{P} & {\left[I<D \mid b_{0}=1, n=i\right]=} \\
& =\mathbf{P}\left[E_{0}^{2}\left\{1+n \alpha+2 \sqrt{\alpha} \sum_{i=1}^{N} b_{i} X_{i 0} Y_{i 0}\right\}+n_{T}<D \mid b_{0}=1, n=i\right] \\
& =\mathbf{P}\left[2 E_{0}^{2} \sqrt{\alpha} \sum_{i=1}^{N} b_{i} X_{i 0} Y_{i 0}+n_{T}<D-E_{0}^{2}(1+n \alpha) \mid b_{0}=1, n=i\right] \\
& =\mathbf{P}\left[\frac{2 E_{0}^{2} \sqrt{\alpha} \sum_{i=1}^{N} b_{i} X_{i 0} Y_{i 0}+n_{T}}{\sqrt{4 E_{0}^{4} n \alpha \mathbf{V}[X Y]+\sigma_{T}^{2}}}<\frac{D-E_{0}^{2}(1+n \alpha)}{\sqrt{4 E_{0}^{4} n \alpha \mathbf{V}[X Y]+\sigma_{T}^{2}}} \mid b_{0}=1, n=i\right]
\end{aligned}
$$




$$
=Q\left(\frac{E_{0}^{2}(1+i \alpha)-D}{\sqrt{4 E_{0}^{4} i \alpha V[X Y]+\sigma_{T}^{2}}}\right)=Q\left(\frac{\left(\mu_{1_{i}}-\mu_{0_{i}}\right)(1+i \alpha)-D}{\sqrt{4\left(\sigma_{1_{i}}^{2}-\sigma_{0_{i}}^{2}\right)+\sigma_{T}^{2}}}\right) .
$$

Assim

$$
\begin{aligned}
& P_{e_{N}}^{\text {compl }}= \\
& =2^{-(N+1)} \sum_{i=0}^{N}\left(\begin{array}{c}
N \\
i
\end{array}\right)\left[Q\left(\frac{D-E_{0}^{2} i \alpha}{\sigma_{T}}\right)+Q\left(\frac{E_{0}^{2}(1+i \alpha)-D}{\sqrt{4 E_{0}^{4} i \alpha \mathbf{V}[X Y]+\sigma_{T}^{2}}}\right)\right] \\
& =2^{-(N+1)} \sum_{i=0}^{N}\left(\begin{array}{c}
N \\
i
\end{array}\right)\left[Q\left(\frac{D-\left(\mu_{1_{i}}-\mu_{0_{i}}\right) i \alpha}{\sigma_{T}}\right)+Q\left(\frac{\left(\mu_{1_{i}}-\mu_{0_{i}}\right)(1+i \alpha)-D}{\sqrt{4\left(\sigma_{1_{i}}^{2}-\sigma_{0_{i}}^{2}\right)+\sigma_{T}^{2}}}\right)\right],
\end{aligned}
$$

onde $\mathbf{V}[X Y]=\frac{1}{2}$ no caso de polarizações alinhadas e $\mathbf{V}[X Y]=\frac{1}{4}$ no caso de polarizações aleatórias uniformes. Fazendo

$$
D=D_{N}=\frac{\mu_{0_{N}}+\mu_{1_{N}}}{2}=\frac{E_{0}^{2}}{2}(1+N \alpha)
$$

tem-se:

$$
\begin{aligned}
P_{e_{N}}^{\text {compl }}= & 2^{-(N+1)} \sum_{i=0}^{N}\left(\begin{array}{c}
N \\
i
\end{array}\right)\left[Q\left(\frac{\left(\mu_{1_{N}}+\mu_{0_{N}}\right)-2\left(\mu_{1_{i}}-\mu_{0_{i}}\right) i \alpha}{\sigma_{T}}\right)+\right. \\
& \left.+Q\left(\frac{2\left(\mu_{1_{i}}-\mu_{0_{i}}\right)(1+i \alpha)-\left(\mu_{1_{N}}+\mu_{0_{N}}\right)}{\sqrt{4\left(\sigma_{1_{i}}^{2}-\sigma_{0_{i}}^{2}\right)+\sigma_{T}^{2}}}\right)\right] \\
= & 2^{-(N+1)} \sum_{i=0}^{N}\left(\begin{array}{c}
N \\
i
\end{array}\right)\left[Q\left(\frac{\left(\mu_{1_{i}}-\mu_{0_{i}}\right)[1+(N-2 i) \alpha]}{\sigma_{T}}\right)+\right. \\
& \left.+Q\left(\frac{\left(\mu_{1_{i}}-\mu_{0_{i}}\right)[1-(N-2 i) \alpha]}{\sqrt{4\left(\sigma_{1_{i}}^{2}-\sigma_{0_{i}}^{2}\right)+\sigma_{T}^{2}}}\right)\right]
\end{aligned}
$$

Definindo

$$
\zeta=\frac{E_{0}^{2}}{\sigma_{T}}=\frac{\left(\mu_{1_{i}}-\mu_{0_{i}}\right)}{\sigma_{T}}
$$


Tem-se

$$
\begin{aligned}
& P_{e_{N}}^{c o m p l}(\zeta)= \\
& \quad=2^{-(N+1)} \sum_{i=0}^{N}\left(\begin{array}{c}
N \\
i
\end{array}\right)\left[Q\left(\frac{\zeta}{2}[1+(N-2 i) \alpha]\right)+Q\left(\frac{\zeta}{2} \frac{[1-(N-2 i) \alpha]}{\sqrt{1+4 \zeta^{2} i \alpha \mathbf{V}[X Y]}}\right)\right]
\end{aligned}
$$

É interessante comparar este resultado com o obtido anteriormente para a probabilidade de erro. É fácil mostrar que

$$
\xi_{N}=\frac{\zeta}{\sqrt{1+\frac{\zeta^{2} N \alpha^{2}}{4}}} .
$$

Fazendo $\alpha_{i}=\alpha \forall i$ na expressão (2.3.23), obtém-se

$$
\begin{aligned}
& P_{e_{N}}^{\text {simpl }}(\zeta)= \\
& =\frac{1}{2}\left[Q\left(\frac{\zeta}{2} \frac{1}{\sqrt{1+\frac{\zeta^{2} N \alpha^{2}}{4}}}\right)+Q\left(\frac{\zeta}{2} \frac{1}{\sqrt{1+\frac{\zeta^{2} N \alpha^{2}}{4}}} \frac{1}{\sqrt{1+\frac{\zeta^{2} N \alpha \mathbf{V}[X Y]}{\left(1+\frac{\zeta^{2} N \alpha^{2}}{4}\right)}}}\right)\right] \\
& =\frac{1}{2}\left[Q\left(\frac{\zeta}{2} \frac{1}{\sqrt{1+\frac{\zeta^{2} N \alpha^{2}}{4}}}\right)+Q\left(\frac{\zeta}{2} \frac{1}{\sqrt{1+\frac{\zeta^{2} N \alpha}{4}(\alpha+4 \mathbf{V}[X Y])}}\right)\right] .
\end{aligned}
$$

Também é interessante comentar a diferença entre as interpretações das variáveis $\zeta$ e $\xi$. A variável $\zeta$ é a relação sinal-ruído ótica e a variável $\xi$ é a relação sinalruído-interferência ótica para o nível 0 . Note que a relação sinal-ruído elétrica é dada por

$$
R S R=\zeta^{2}=\xi_{0}^{2}
$$

enquanto a relação sinal-ruído-interferência elétrica para o nível 0 é dada por

$$
R S R I_{0_{N}}=\xi_{N}^{2}
$$

Note que nos gráficos apresentados neste trabalho, as grandezas utilizadas no eixo horizontal são sempre relações sinal-ruído elétricas. 


\subsection{Comparação entre os níveis de chão}

Pode-se calcular os valores de chão previstos por cada um dos modelos analisados:

$$
B E R_{\text {floor }}^{\text {simpl }}(N)=\lim _{\zeta \rightarrow \infty} P_{e_{N}}^{\text {simpl }}(\zeta)=\frac{1}{2}\left[Q\left(\frac{1}{\alpha \sqrt{N}}\right)+Q\left(\frac{1}{\sqrt{N \alpha(\alpha+4 \mathbf{V}[X Y])}}\right)\right]
$$

e

$B E R_{\text {floor }}^{\text {compl }}(N)=\lim _{\zeta \rightarrow \infty} P_{e_{N}}^{\text {compl }}(\zeta)=2^{-(N+1)} \sum_{i=0}^{N}\left[\left(\begin{array}{c}N \\ i\end{array}\right) Q\left(\frac{[1-(N-2 i) \alpha]}{4 \sqrt{i \alpha \mathbf{V}[X Y]}}\right)\right]$.

Usando o teorema de DeMoivre-Laplace [35] na expressão (2.5.2), pode-se escrever a seguinte aproximação:

$$
B E R_{\text {floor }}^{\text {compl }}(N) \cong \sum_{i=0}^{N} \frac{1}{\sqrt{2 \pi N}} e^{\frac{-(2 i-N)^{2}}{2 N}} Q\left(\frac{[1-(N-2 i) \alpha]}{4 \sqrt{i \alpha \mathbf{V}[X Y]}}\right)
$$

que é uma expressão melhor de se utilizar, do ponto de vista computacional, para grandes valores de N. Uma comparação visual entre os gráficos das probabilidades de erro calculadas pelo modelo "simplificado" e pelo modelo "completo", mostra que as curvas se comportam de maneira muito semelhante, isto é, a forma de "S" é muito parecida, e em ambas se deve à presença de uma função "Q". Como a expressão do modelo "completo" da probabilidade de erro é computacionalmente muito intensa para valores grandes de $\mathrm{N}$, aqui se propõe um novo método para o cálculo da probabilidade de erro, que consiste em se utilizar a expressão do método simplificado com um valor de $\mathrm{N}$ equivalente dado pela expressão

$$
N_{e q v}=\frac{1}{\alpha(\alpha+4 \mathbf{V}[X Y])}\left[Q^{-1}\left(2 B E R_{\text {floor }}^{\text {compl }}(N)\right)\right]^{2}
$$

Nesta expressão, $Q^{-1}(x)$ é a inversa da função $Q(x)$, e foi obtida a partir da 
expressão (2.5.1) assumindo que a contribuição do segundo termo é maior que a contribuição do primeiro e desprezando este no cálculo. A figura 2.2 mostra os resultados obtidos com este modelo.

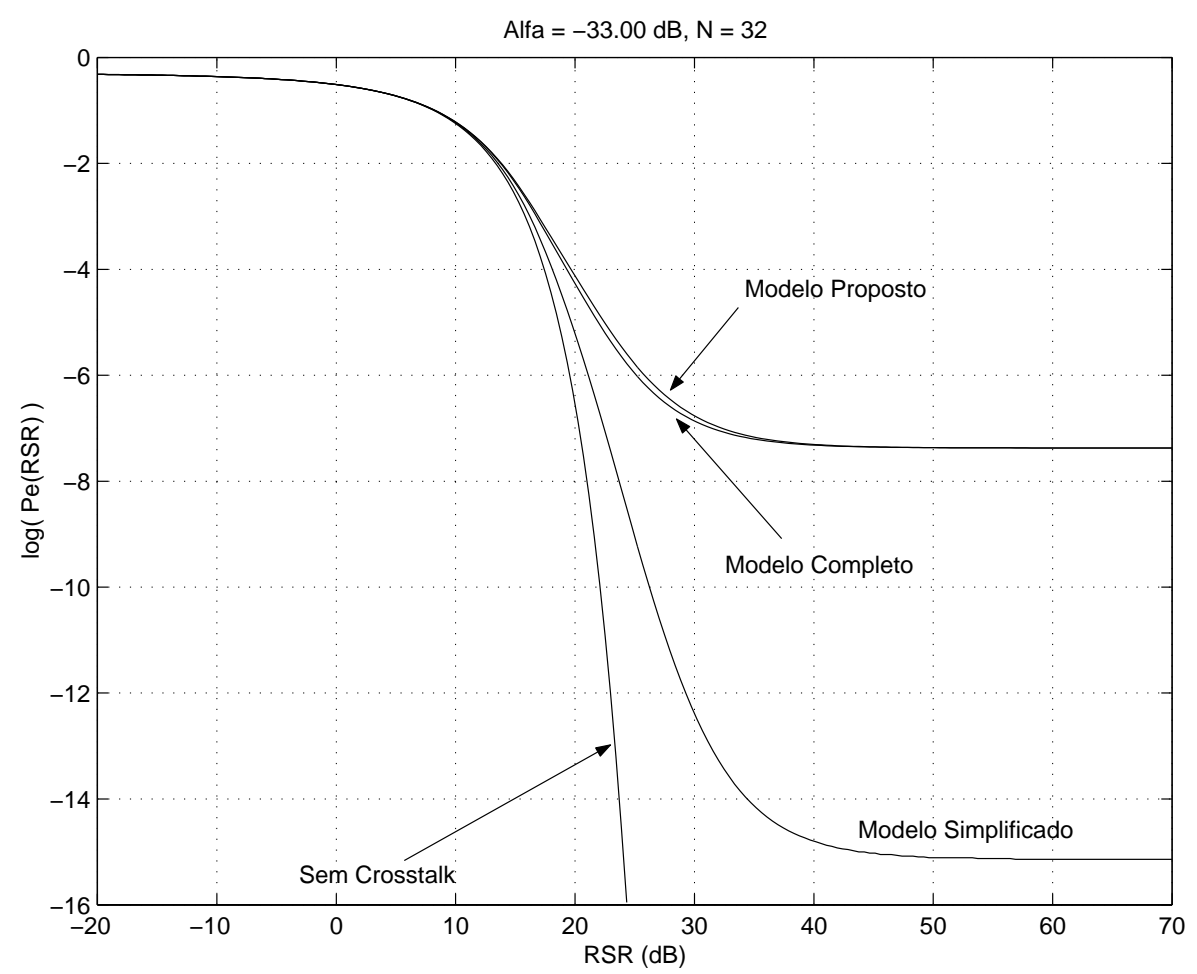

Figura 2.2: Comparação entre os valores de probabilidade de erro sem crosstalk, o modelo Gaussiano simplificado, o modelo Gaussiano completo e o modelo proposto

A interpretação deste resultado é que provavelmente existe uma parcela da soma da expressão da probabilidade de erro completa que deve estar predominando sobre as outras. A fim de testar esta idéia, pode se calcular aproximadamente a qual índice 
corresponde o número de crosstalks equivalente pela expressão

$$
\begin{gathered}
\frac{1}{2} Q\left(\frac{[1-(N-2 i) \alpha]}{4 \sqrt{i \alpha \mathbf{V}[X Y]}}\right) \approx \frac{1}{2} Q\left(\frac{1}{4 \sqrt{i \alpha \mathbf{V}[X Y]}}\right)=B E R_{\text {floor }}^{\text {compl }}(N) \Rightarrow \\
i \approx \frac{1}{16 \alpha \mathbf{V}[X Y]\left[Q^{-1}\left(2 B E R_{\text {floor }}^{\text {compl }}(N)\right)\right]^{2}} \approx \frac{N_{\text {eqv }}}{4}
\end{gathered}
$$

O gráfico da figura 2.3 mostra que, de modo grosseiro, $N_{e q v} \approx 2 N$ quando $\mathrm{N}$ é grande, o que significa segundo a expressão $(2.5 .5)$ que $i \approx \frac{N}{2}$. Isto mostra que o caso predominante na expressão da probabilidade de erro é aquele em que metade dos bits de crosstalk estão interferindo. O que é bastante razoável, uma vez que o número de crosstalks é uma variável aleatória binomial com parâmetros $\left(p=\frac{1}{2}, N\right)$, o que dá uma média de $\frac{N}{2}$.

Como as expressões (2.3.33) e (2.3.34) foram deduzidas para o caso simplificado, é possível utilizá-las para uma aproximação mais precisa do cálculo das penalidades de potência, substituindo o valor de $\mathrm{N}$ pelo de $\mathrm{N}$ equivalente

$$
\begin{aligned}
P P_{N} & =-5 \log \left[1-q^{2} N_{e q v} \alpha(\alpha+4 \mathbf{V}[X Y])\right] \\
& \approx-5 \log \left[1-2 q^{2} N \alpha(\alpha+4 \mathbf{V}[X Y])\right]
\end{aligned}
$$

Métodos numéricos para inverter as expressões (2.4.8) e (2.4.10) para um dado valor de probabilidade de erro podem facilmente ser implementados. Neste caso a penalidade de potência para esta probabilidade de erro pode ser calculada por

$$
P P_{N}=10 \log \left(\frac{\left(\mu_{1_{N}}-\mu_{0_{N}}\right)}{\left(\mu_{1_{0}}-\mu_{0_{0}}\right)}\right)=10 \log \left(\frac{\zeta_{N}\left(P_{0}\right)}{\zeta_{0}\left(P_{0}\right)}\right)=5 \log \left(\frac{R S R_{N}}{R S R_{0}}\right)
$$

onde

$$
\zeta_{i}\left(P_{0}\right)=P_{e_{i}}^{-1}\left(P_{0}\right)
$$




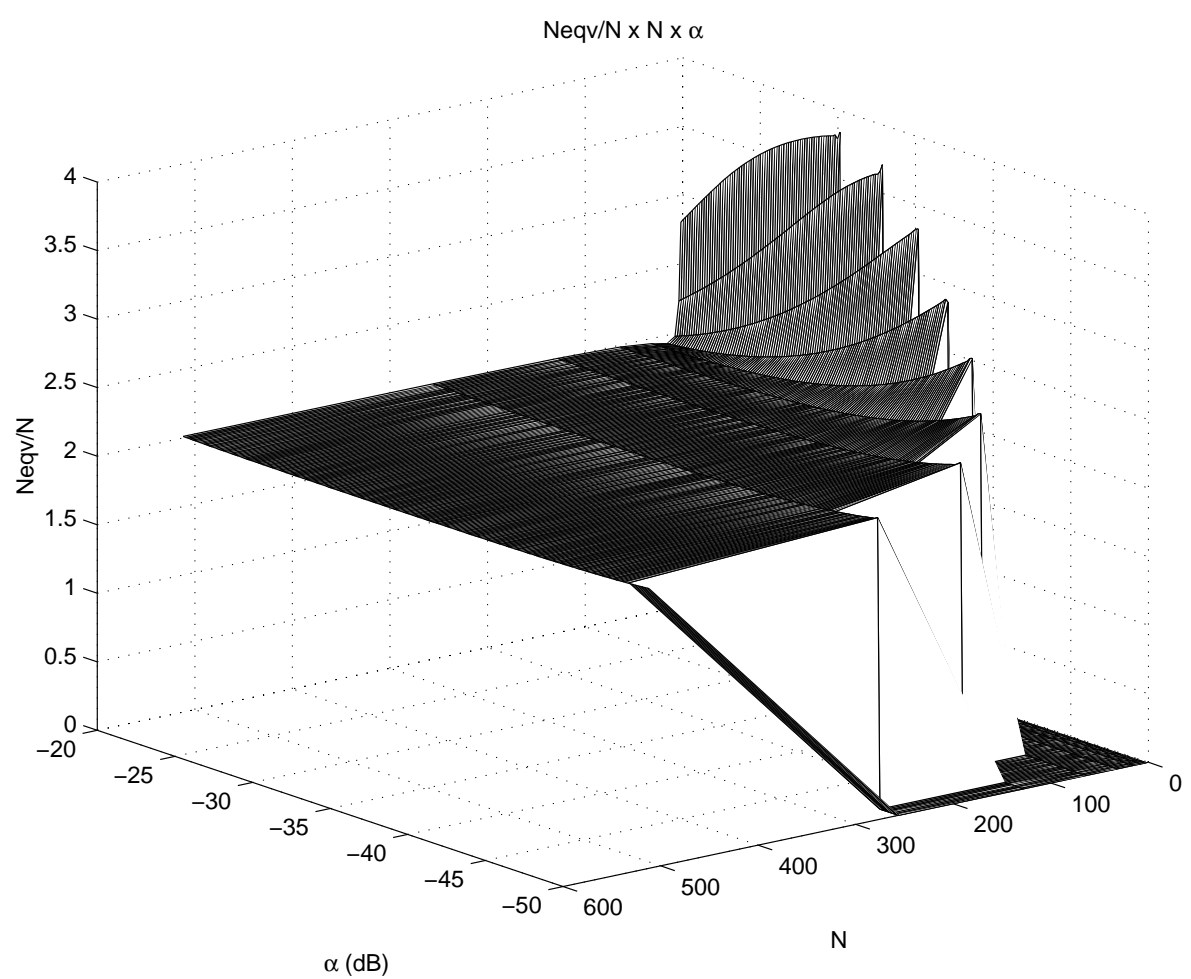

Figura 2.3: A razão entre $\mathrm{N}$ equivalente e $\mathrm{N}$ é aproximadamente 2 na maior parte da região

\subsection{Usando outros níveis de decisão}

Se o nível de decisão utilizado for [1]

$$
D_{N, \text { prox }}=\frac{\sigma_{0_{N}} \mu_{1_{N}}+\sigma_{1_{N}} \mu_{0_{N}}}{\sigma_{0_{N}}+\sigma_{1_{N}}}
$$

a probabilidade de erro se reduz a

$$
P_{e_{N}, \text { prox }}=Q\left(\frac{\mu_{1_{N}}-\mu_{0_{N}}}{\sigma_{0_{N}}+\sigma_{1_{N}}}\right) .
$$


Note que este caso corresponde a um nível de decisão próximo ao ótimo, mas não ao ótimo ( vide apêndice III ). O ponto de decisão ótimo pode ser calculado derivando a expressão (2.3.20) em relação ao nível de decisão, igualando o resultado a zero e achando as raizes, tem-se

$$
D_{\text {opt }}=\frac{1}{\left(\sigma_{1}^{2}-\sigma_{0}^{2}\right)}\left[\sigma_{1}^{2} \mu_{0}-\sigma_{0}^{2} \mu_{1} \pm \sigma_{0} \sigma_{1}\left|\left(\mu_{1}-\mu_{0}\right)\right| \sqrt{1+\frac{2\left(\sigma_{1}^{2}-\sigma_{0}^{2}\right)}{\left(\mu_{1}-\mu_{0}\right)^{2}} \ln \left(\frac{\sigma_{1}}{\sigma_{0}}\right)}\right] .
$$

Pode ser mostrado que nos casos em que o termo dentro do radical for muito próximo de 1, a expressão (2.6.3) com a raiz de sinal positivo se reduz à expressão (2.6.1). A raiz de sinal negativo se refere a um ponto de máximo, o que não deixa de ser um resultado curioso: uma análise cuidadosa da forma da função probabilidade de erro revela que existem pontos de decisão para os quais a probabilidade de erro é maior que 0.5, e além disso, existe um ponto em que essa probabilidade de erro é máxima. Pode ser mostrado também que apesar do ponto de decisão dado por (2.6.1) não ser ótimo, este valor sempre conduz à probabilidades de erro menores que as que o ponto de decisão dado por (2.3.21) fornece ( vide apêndice IV ). Apenas para manter o desenvolvimento completo se a expressão (2.6.3) para o ponto de decisão tivesse sido utilizada, a expressão da probabilidade de erro seria

$$
P_{e_{N}, o p t}=\frac{1}{2}\left\{Q\left[\frac{\left(\mu_{1_{N}}-\mu_{0_{N}}\right) \sigma_{1_{N}}}{\sigma_{1_{N}}^{2}-\sigma_{0_{N}}^{2}}\left(-\frac{\sigma_{0}}{\sigma_{1}} \pm R\right)\right]+Q\left[\frac{\left(\mu_{1_{N}}-\mu_{0_{N}}\right) \sigma_{0_{N}}}{\sigma_{1_{N}}^{2}-\sigma_{0_{N}}^{2}}\left(\frac{\sigma_{1}}{\sigma_{0}} \pm R\right)\right]\right\}
$$

onde $\mathrm{R}$ é o termo dentro do radical da equação 2.6 .3 e

$$
\frac{\sigma_{1_{N}}}{\sigma_{0_{N}}}=\sqrt{1+\xi_{N}^{2} N \alpha \mathbf{V}}=\sqrt{\frac{1+\frac{\zeta^{2} N \alpha}{4}(\alpha+4 \mathbf{V})}{1+\frac{\zeta^{2} N \alpha^{2}}{4}}}
$$

É possível então fazer um desenvolvimento análogo ao realizado anteriormente que produza uma expressão analítica para a penalidade de potência. Reescrevendo a 
expressão (2.6.2) em função de $\xi_{N}$, obtém-se

$$
P_{e_{N}, p r o x}^{s i m p l}\left(\xi_{N}\right)=Q\left(\frac{\xi_{N}}{\left(1+\sqrt{1+\xi_{N}^{2} \sum_{i=1}^{N} \alpha_{i} \mathbf{V}\left[X_{i 0} Y_{i 0}\right]}\right)}\right) .
$$

Seja $q_{\text {prox }}$ tal que $Q\left(q_{\text {prox }}\right)=10^{-9}$, o que dá $q_{\text {prox }} \approx 5.9978$. Então,

$$
q_{\text {prox }}=\left(\frac{\mu_{1_{N}}-\mu_{0_{N}}}{\sigma_{1_{N}}+\sigma_{0_{N}}}\right)=\left(\frac{\mu_{1_{0}}-\mu_{0_{0}}}{\sigma_{1_{0}}+\sigma_{0_{0}}}\right),
$$

o que quer dizer que

$$
\frac{\left(\mu_{1_{N}}-\mu_{0_{N}}\right)}{\left(\mu_{1_{0}}-\mu_{0_{0}}\right)}=\frac{\left(\sigma_{1_{N}}+\sigma_{0_{N}}\right)}{\left(\sigma_{1_{0}}+\sigma_{0_{0}}\right)} .
$$

Deste modo,

$$
P P_{N}=10 \log \left(\frac{\left(\mu_{1_{N}}-\mu_{0_{N}}\right)}{\left(\mu_{1_{0}}-\mu_{0_{0}}\right)}\right)=10 \log \left(\frac{\left(\sigma_{1_{N}}+\sigma_{0_{N}}\right)}{\left(\sigma_{1_{0}}+\sigma_{0_{0}}\right)}\right)=-5 \log \left(\frac{\left(\sigma_{1_{0}}+\sigma_{0_{0}}\right)^{2}}{\left(\sigma_{1_{N}}+\sigma_{0_{N}}\right)^{2}}\right) .
$$

Quando $N=0, \sigma_{1_{0}}+\sigma_{0_{0}}=2 \sigma_{T}$. Quando $N>0$, das expressões (2.3.12) e (2.3.13) tem-se

$$
\begin{aligned}
\left(\sigma_{1_{N}}+\sigma_{0_{N}}\right)= & \sqrt{\frac{\left(\mu_{1}-\mu_{0}\right)^{2}}{4} \sum_{i=1}^{N}\left[4 \alpha_{i} V\left[X_{i 0} Y_{i 0}\right]+\alpha_{i}^{2}\right]+\sigma_{T}^{2}} \\
& +\sqrt{\frac{\left(\mu_{1}-\mu_{0}\right)^{2}}{4} \sum_{i=1}^{N} \alpha_{i}^{2}+\sigma_{T}^{2}}
\end{aligned}
$$

A partir desta relação, pode-se mostrar que ( vide apêndice II )

$$
\sigma_{T}^{2}=\frac{\left[\left(\sigma_{1_{N}}+\sigma_{0_{N}}\right)^{2}-\left(\mu_{1_{N}}-\mu_{0_{N}}\right)^{2} \sum_{i=1}^{N} \alpha_{i} V\left[X_{i 0} Y_{i 0}\right]\right]^{2}}{4\left(\sigma_{1_{N}}+\sigma_{0_{N}}\right)^{2}}-\left(\mu_{1_{N}}-\mu_{0_{N}}\right)^{2} \sum_{i=1}^{N} \alpha_{i}^{2} .
$$

Substituindo (2.6.11) em (2.6.9)

$$
P P_{N, p r o x}=
$$




$$
\begin{aligned}
=-5 \log \left[\left(1-\frac{\left(\mu_{1_{N}}-\mu_{0_{N}}\right)^{2}}{\left(\sigma_{1_{N}}+\sigma_{0_{N}}\right)^{2}} \sum_{i=1}^{N} \alpha_{i} \mathbf{V}\left[X_{i 0} Y_{i 0}\right]\right)^{2}-\frac{\left(\mu_{1_{N}}-\mu_{0_{N}}\right)^{2}}{\left(\sigma_{1_{N}}+\sigma_{0_{N}}\right)^{2}} \sum_{i=1}^{N} \alpha_{i}^{2}\right] \\
=-5 \log \left[\left(1-q_{\text {prox }}^{2} \sum_{i=1}^{N} \alpha_{i} \mathbf{V}\left[X_{i 0} Y_{i 0}\right]\right)^{2}-q_{\text {prox }}^{2} \sum_{i=1}^{N} \alpha_{i}^{2}\right] \\
=-5 \log \left[\left(1+q_{\text {prox }}^{4} \sum_{i=1}^{N} \sum_{j=1}^{N} \alpha_{i} \alpha_{j} \mathbf{V}\left[X_{i 0} Y_{i 0}\right] \mathbf{V}\left[X_{j 0} Y_{j 0}\right]\right)\right. \\
\left.-q_{\text {prox }}^{2}\left\{\sum_{i=1}^{N} \alpha_{i}\left(2 \mathbf{V}\left[X_{i 0} Y_{i 0}\right]+\alpha_{i}\right)\right\}\right]
\end{aligned}
$$

Fazendo $\alpha_{i}=\alpha \forall i$ e $V=V\left[X_{i 0} Y_{i 0}\right]$, tem-se

$$
P P_{N, p r o x}=-5 \log \left[\left(1+q_{\text {prox }}^{4} N^{2} \alpha^{2} \mathbf{V}^{2}\right)-q_{\text {prox }}^{2} N \alpha(2 \mathbf{V}+\alpha)\right]
$$

O que dá para polarizações alinhadas

$$
P P_{N, \text { prox }}=-5 \log \left[\left(1+\frac{q_{\text {prox }}^{4} N^{2} \alpha^{2}}{4}\right)-q_{\text {prox }}^{2} N \alpha(1+\alpha)\right]
$$

e para polarizações aleatórias uniformes

$$
P P_{N, \text { prox }}=-5 \log \left[\left(1+\frac{q_{\text {prox }}^{4} N^{2} \alpha^{2}}{16}\right)-q_{\text {prox }}^{2} N \alpha\left(\frac{1}{2}+\alpha\right)\right] .
$$

A expressão (2.6.13) para a penalidade de potência não sofre do mesmo problema da expressão (2.3.30), uma vez que nenhum termo foi desprezado. Desde que o nível de decisão adotado seja dado por (2.6.1), a expressão acima é exata.

Para o caso da aproximação Gaussiana completa usando o nível de decisão dado por (2.6.1), as expressões (2.3.10) e (2.3.11), tem-se da relação (2.4.4),

$$
\begin{aligned}
& P_{e_{N}, p r o x}^{\text {compl }}(\zeta)= \\
& \quad=2^{-(N+1)} \sum_{i=1}^{N}\left(\begin{array}{c}
N \\
i
\end{array}\right)\left[Q\left(\frac{D-E_{0}^{2} i \alpha}{\sigma_{T}}\right)+Q\left(\frac{E_{0}^{2}(1+i \alpha)-D}{\sigma_{T} \sqrt{1+\frac{4 E_{0}^{4} i \alpha \mathbf{V}[X Y]}{\sigma_{T}^{2}}}}\right)\right]
\end{aligned}
$$




$$
\begin{aligned}
& =2^{-(N+1)} \sum_{i=1}^{N}\left(\begin{array}{c}
N \\
i
\end{array}\right)\left[Q\left(\frac{D}{\sigma_{T}}-\zeta i \alpha\right)+Q\left(\frac{\zeta(1+i \alpha)-\frac{D}{\sigma_{T}}}{\sqrt{1+4 \zeta^{2} i \alpha \mathbf{V}[X Y]}}\right)\right] \\
& \frac{D}{\sigma_{T}}=\frac{\sigma_{0_{N}} \mu_{1_{N}}+\sigma_{1_{N}} \mu_{0_{N}}}{\sigma_{T}\left(\sigma_{0_{N}}+\sigma_{1_{N}}\right)}=\frac{\sigma_{0_{N}}\left(\mu_{1_{N}}-\mu_{0_{N}}\right)\left(1+\frac{N \alpha}{2}\right)+\sigma_{1_{N}}\left(\mu_{1_{N}}-\mu_{0_{N}}\right) \frac{N \alpha}{2}}{\sigma_{T}\left(\sigma_{0_{N}}+\sigma_{1_{N}}\right)}= \\
& \zeta\left[\frac{\sigma_{0_{N}}}{\sigma_{0_{N}}+\sigma_{1_{N}}}+\frac{N \alpha}{2}\right] \text {, } \\
& \frac{D}{\sigma_{T}}-\zeta i \alpha=\frac{\zeta}{2}\left[\frac{2}{1+\frac{\sigma_{1}}{\sigma_{0_{N}}}}+(N-2 i) \alpha\right] \\
& \zeta(1+i \alpha)-\frac{D}{\sigma_{T}}=\frac{\zeta}{2}\left[2-\frac{2}{1+\frac{\sigma_{1}}{\sigma_{0}}}-(N-2 i) \alpha\right], \\
& \frac{\sigma_{1}}{\sigma_{0}}=\sqrt{\frac{1+\zeta^{2} N \frac{\alpha}{4}(\alpha+4 \mathbf{V}[X Y])}{1+\zeta^{2} N \frac{\alpha}{4}}}, \lim _{\zeta \rightarrow \infty} \frac{\sigma_{1}}{\sigma_{0}}=\sqrt{\alpha+4 \mathbf{V}[X Y]},
\end{aligned}
$$

Finalmente,

$$
\begin{aligned}
& P_{e_{N}, p r o x}^{\text {compl }}(\zeta)= \\
& =2^{-(N+1)} \sum_{i=0}^{N}\left(\begin{array}{c}
N \\
i
\end{array}\right)\left(Q\left[\frac{\zeta}{2}\left(\frac{2}{1+\sqrt{\frac{1+\zeta^{2} N \frac{\alpha}{4}(\alpha+4 \mathbf{V})}{1+\zeta^{2} N \frac{\alpha}{4}}}}+(N-2 i) \alpha\right)\right]\right. \\
& \left.\quad+Q\left[\frac{\zeta}{2 \sqrt{1+4 \zeta^{2} i \alpha \mathbf{V}}}\left(2-\frac{2}{1+\sqrt{\frac{1+\zeta^{2} N \frac{\alpha}{4}(\alpha+4 \mathbf{V})}{1+\zeta^{2} N \frac{\alpha}{4}}}}-(N-2 i) \alpha\right)\right]\right)
\end{aligned}
$$

Reescrevendo a expressão (2.6.6) em função de $\zeta$, tem-se

$$
P_{e_{N}, p r o x}^{\text {simpl }}(\zeta)=Q\left[\frac{\zeta}{\left(\sqrt{1+\zeta^{2} \sum_{i=1}^{N} \frac{\alpha_{i}^{2}}{4}}+\sqrt{1+\zeta^{2} \sum_{i=1}^{N} \frac{\alpha_{i}}{4}\left(\alpha_{i}+4 \mathbf{V}\left[X_{i 0} Y_{i 0}\right]\right)}\right)}\right]
$$


Os novos valores de chão são então

$$
B E R_{\text {floor }, \text { prox }}^{\text {simpl }}(N)=\lim _{\zeta \rightarrow \infty} P_{e_{1}, p r o x}^{\text {simpl }}(\zeta)=Q\left[\frac{2}{\sqrt{N}(\alpha+\sqrt{\alpha(\alpha+4 \mathbf{V}[X Y])})}\right]
$$

e

$$
B E R_{\text {floor }, \text { prox }}^{\text {compl }}(N)=2^{-(N+1)} \sum_{i=0}^{N}\left(\begin{array}{c}
N \\
i
\end{array}\right)\left\{Q\left[\frac{\left(2-\frac{2}{1+\sqrt{\alpha+4 \mathbf{V}[X Y]}}-(N-2 i) \alpha\right)}{4 \sqrt{i \alpha \mathbf{V}[X Y]}}\right]\right\} \text {. }
$$

A fim de utilizar a mesma idéia utilizada anteriormente para calcular a expressão da probabilidade de erro no caso completo aproveitando a expressão do caso simplificado, a expressão de $\mathrm{N}$ equivalente deve ser modificada para

$$
N_{\text {eqv }, \text { prox }}=\frac{4}{\left[(\alpha+\sqrt{\alpha(\alpha+4 \mathbf{V}[X Y])}) Q^{-1}\left(B E R_{\text {floor }, \text { prox }}^{\text {compl }}(N)\right)\right]^{2}} .
$$

\subsection{Apêndice I - Variância do produto}

Supondo que X e Y são variáveis aleatórias independentes

$$
\begin{aligned}
\mathbf{V}[X Y] & =\mathbf{E}\left[(X Y)^{2}\right]-\mathbf{E}^{2}[X Y]=\mathbf{E}\left[X^{2} Y^{2}\right]-\mathbf{E}^{2}[X] \mathbf{E}^{2}[Y] \\
& =\mathbf{E}\left[X^{2}\right] \mathbf{E}\left[Y^{2}\right]-\mathbf{E}^{2}[X] \mathbf{E}^{2}[Y] \\
& =\mathbf{E}\left[X^{2}\right] \mathbf{E}\left[Y^{2}\right]-\mathbf{E}^{2}[X] \mathbf{E}^{2}[Y]-\mathbf{E}^{2}[X] \mathbf{E}\left[Y^{2}\right]+\mathbf{E}^{2}[X] \mathbf{E}\left[Y^{2}\right] \\
& =\left(\mathbf{E}\left[X^{2}\right]-\mathbf{E}^{2}[X]\right) \mathbf{E}\left[Y^{2}\right]+\mathbf{E}^{2}[X]\left(\mathbf{E}\left[Y^{2}\right]-\mathbf{E}^{2}[Y]\right) \\
& =\mathbf{V}[X] \mathbf{E}\left[Y^{2}\right]+\mathbf{E}^{2}[X] \mathbf{V}[Y] \\
& =\mathbf{V}[X] \mathbf{E}\left[Y^{2}\right]+\mathbf{E}^{2}[X] \mathbf{V}[Y]-\mathbf{V}[X] \mathbf{E}^{2}[Y]+\mathbf{V}[X] \mathbf{E}^{2}[Y] \\
& =\mathbf{V}[X]\left(\mathbf{E}\left[Y^{2}\right]-\mathbf{E}^{2}[Y]\right)+\mathbf{V}[X] \mathbf{E}^{2}[Y]+\mathbf{E}^{2}[X] \mathbf{V}[Y] \\
& =\mathbf{V}[X] \mathbf{V}[Y]+\mathbf{V}[X] \mathbf{E}^{2}[Y]+\mathbf{E}^{2}[X] \mathbf{V}[Y]
\end{aligned}
$$




\subsection{Apêndice II - Variância do ruído térmico}

$$
\begin{aligned}
& \left(\sigma_{1}+\sigma_{0}\right)=\sqrt{k_{1}^{2}+\sigma_{T}^{2}}+\sqrt{k_{0}^{2}+\sigma_{T}^{2}} \\
\Rightarrow \quad & \left(\sigma_{1}+\sigma_{0}\right)^{2}=\left(k_{1}^{2}+\sigma_{T}^{2}\right)+\left(k_{0}^{2}+\sigma_{T}^{2}\right)+2 \sqrt{\left(k_{1}^{2}+\sigma_{T}^{2}\right)\left(k_{0}^{2}+\sigma_{T}^{2}\right)} \\
\Rightarrow & 2 \sqrt{\left(k_{1}^{2}+\sigma_{T}^{2}\right)\left(k_{0}^{2}+\sigma_{T}^{2}\right)}=\left(\sigma_{1}+\sigma_{0}\right)^{2}-\left(k_{1}^{2}+k_{0}^{2}+2 \sigma_{T}^{2}\right) \\
\Rightarrow \quad & 4\left(k_{1}^{2} k_{0}^{2}+\sigma_{T}^{2}\left(k_{1}^{2}+k_{0}^{2}\right)+\sigma_{T}^{4}\right)= \\
& \left(\sigma_{1}+\sigma_{0}\right)^{4}+\left(k_{1}^{2}+k_{0}^{2}+2 \sigma_{T}^{2}\right)^{2}-2\left(\sigma_{1}+\sigma_{0}\right)^{2}\left(k_{1}^{2}+k_{0}^{2}+2 \sigma_{T}^{2}\right)= \\
& \left(\sigma_{1}+\sigma_{0}\right)^{4}+\left(k_{1}^{2}+k_{0}^{2}\right)^{2}+4 \sigma_{T}^{2}\left(k_{1}^{2}+k_{0}^{2}\right)+4 \sigma_{T}^{4}-2\left(\sigma_{1}+\sigma_{0}\right)^{2}\left(k_{1}^{2}+k_{0}^{2}+2 \sigma_{T}^{2}\right) \\
\Rightarrow \quad & 4 \sigma_{T}^{2}\left(\sigma_{1}+\sigma_{0}\right)^{2}+4 k_{1}^{2} k_{0}^{2}-\left(\sigma_{1}+\sigma_{0}\right)^{4}-\left(k_{1}^{2}+k_{0}^{2}\right)^{2}+2\left(\sigma_{1}+\sigma_{0}\right)^{2}\left(k_{1}^{2}+k_{0}^{2}\right)=0 \\
\Rightarrow \quad & 4 \sigma_{T}^{2}\left(\sigma_{1}+\sigma_{0}\right)^{2}-\left(\sigma_{1}+\sigma_{0}\right)^{4}-\left(k_{1}^{2}-k_{0}^{2}\right)^{2}+ \\
& 2\left(\sigma_{1}+\sigma_{0}\right)^{2}\left(k_{1}^{2}+k_{0}^{2}\right) \pm 2\left(\sigma_{1}+\sigma_{0}\right)^{2}\left(k_{1}^{2}-k_{0}^{2}\right)=0 \\
\Rightarrow & 4 \sigma_{T}^{2}\left(\sigma_{1}+\sigma_{0}\right)^{2}-\left[\left(\sigma_{1}+\sigma_{0}\right)^{4}-\left(k_{1}^{2}-k_{0}^{2}\right)^{2}\right]^{2}+2\left(\sigma_{1}+\sigma_{0}\right)^{2} k_{0}^{2}=0 \\
& \quad \sigma_{T}^{2}=\frac{\left[\left(\sigma_{1}+\sigma_{0}\right)^{4}-\left(k_{1}^{2}-k_{0}^{2}\right)^{2}\right]^{2}}{4\left(\sigma_{1}+\sigma_{0}\right)^{2}} \\
&
\end{aligned}
$$

\subsection{Apêndice III - Ponto de decisão ótimo}

$$
\begin{aligned}
& P_{e}(D)=\frac{1}{2}\left[Q\left(\frac{D-\mu_{0}}{\sigma_{0}}\right)+Q\left(\frac{\mu_{1}-D}{\sigma_{1}}\right)\right] \\
& \frac{d P_{e}(D)}{d D}=\frac{1}{2 \sqrt{2 \pi}}\left[\frac{1}{\sigma_{0} e^{-\frac{\left(D-\mu_{0}\right)^{2}}{2 \sigma_{0}^{2}}}}-\frac{1}{\sigma_{1} e^{-\frac{\left(\mu_{1}-D\right)^{2}}{2 \sigma_{1}^{2}}}}\right]=0 \\
& \Rightarrow \frac{\sigma_{1}}{\sigma_{0}} e^{-\frac{\left(D-\mu_{0}\right)^{2}}{2 \sigma_{0}^{2}}}=e^{-\frac{\left(\mu_{1}-D\right)^{2}}{2 \sigma_{1}^{2}}} \Rightarrow e^{\ln \left(\frac{\sigma_{1}}{\sigma_{0}}\right)-\frac{\left(D-\mu_{0}\right)^{2}}{2 \sigma_{0}^{2}}}=e^{-\frac{\left(\mu_{1}-D\right)^{2}}{2 \sigma_{1}^{2}}} \\
& \Rightarrow \frac{\left(D-\mu_{0}\right)^{2}}{2 \sigma_{0}^{2}}-\frac{\left(\mu_{1}-D\right)^{2}}{2 \sigma_{1}^{2}}-\ln \left(\frac{\sigma_{1}}{\sigma_{0}}\right)=0
\end{aligned}
$$




$$
\begin{aligned}
& \Rightarrow \sigma_{1}^{2}\left(D-\mu_{0}\right)^{2}-\sigma_{0}^{2}\left(\mu_{1}-D\right)^{2}-2 \sigma_{0}^{2} \sigma_{1}^{2} \ln \left(\frac{\sigma_{1}}{\sigma_{0}}\right)=0 \\
& \Rightarrow\left(\sigma_{1}^{2}-\sigma_{0}^{2}\right) D^{2}-2\left(\sigma_{1}^{2} \mu_{0}-\sigma_{0}^{2} \mu_{1}\right) D+\sigma_{1}^{2} \mu_{0}^{2}-\sigma_{0}^{2} \mu_{1}^{2}-2 \sigma_{0}^{2} \sigma_{1}^{2} \ln \left(\frac{\sigma_{1}}{\sigma_{0}}\right)=0 \\
& \Rightarrow D=\frac{2\left(\sigma_{1}^{2} \mu_{0}-\sigma_{0}^{2} \mu_{1}\right) \pm \sqrt{\begin{array}{l}
4\left(\sigma_{1}^{2} \mu_{0}-\sigma_{0}^{2} \mu_{1}\right)^{2}- \\
4\left(\sigma_{1}^{2}-\sigma_{0}^{2}\right)\left[\sigma_{1}^{2} \mu_{0}^{2}-\sigma_{0}^{2} \mu_{1}^{2}-2 \sigma_{0}^{2} \sigma_{1}^{2} \ln \left(\frac{\sigma_{1}}{\sigma_{0}}\right)\right]
\end{array}}}{2\left(\sigma_{1}^{2}-\sigma_{0}^{2}\right)} \\
& \Rightarrow D=\frac{\sigma_{1}^{2} \mu_{0}-\sigma_{0}^{2} \mu_{1} \pm \sqrt{\begin{array}{l}
\sigma_{1}^{4} \mu_{0}^{2}-2 \sigma_{0}^{2} \sigma_{1}^{2} \mu_{0} \mu_{1}+\sigma_{0}^{4} \mu_{1}^{2}-\sigma_{1}^{4} \mu_{0}^{2}+\sigma_{0}^{2} \sigma_{1}^{2} \mu_{1}^{2}+ \\
2 \sigma_{0}^{2} \sigma_{1}^{4} \ln \left(\frac{\sigma_{1}}{\sigma_{0}}\right)+\sigma_{0}^{2} \sigma_{1}^{2} \mu_{0}^{2}-\sigma_{0}^{4} \mu_{1}^{2}-2 \sigma_{0}^{4} \sigma_{1}^{2} \ln \left(\frac{\sigma_{1}}{\sigma_{0}}\right)
\end{array}}}{\left(\sigma_{1}^{2}-\sigma_{0}^{2}\right)} \\
& \begin{array}{l}
\Rightarrow D=\frac{\sigma_{1}^{2} \mu_{0}-\sigma_{0}^{2} \mu_{1} \pm \sigma_{0} \sigma_{1} \sqrt{-2 \mu_{0} \mu_{1}+\mu_{1}^{2}+\mu_{0}^{2}+2\left(\sigma_{1}^{2}-\sigma_{0}^{2}\right) \ln \left(\frac{\sigma_{1}}{\sigma_{0}}\right)}}{\left(\sigma_{1}^{2}-\sigma_{0}^{2}\right)} \\
\Rightarrow D=\frac{1}{\left(\sigma_{1}^{2}-\sigma_{0}^{2}\right)}\left[\sigma_{1}^{2} \mu_{0}-\sigma_{0}^{2} \mu_{1} \pm \sigma_{0} \sigma_{1}\left|\left(\mu_{1}-\mu_{0}\right)\right| \sqrt{1+2 \frac{\left(\sigma_{1}^{2}-\sigma_{0}^{2}\right)}{\left(\mu_{1}-\mu_{0}\right)^{2}} \ln \left(\frac{\sigma_{1}}{\sigma_{0}}\right)}\right]
\end{array}
\end{aligned}
$$

Assumindo que $\mu_{1}>\mu_{0}$, se o termo dentro do radical for muito próximo de 1 , a expressão se reduz a

$$
\begin{aligned}
D & =\frac{\sigma_{1}^{2} \mu_{0}-\sigma_{0}^{2} \mu_{1} \pm \sigma_{0} \sigma_{1}\left(\mu_{1}-\mu_{0}\right)}{\left(\sigma_{1}^{2}-\sigma_{0}^{2}\right)}=\frac{\sigma_{1}\left(\sigma_{1} \mu_{0} \pm \sigma_{0} \mu_{1}\right)-\sigma_{0}\left(\sigma_{1} \mu_{0} \pm \sigma_{0} \mu_{1}\right)}{\left(\sigma_{1}-\sigma_{0}\right)\left(\sigma_{1}+\sigma_{0}\right)} \\
& \Rightarrow D=\frac{\sigma_{1} \mu_{0} \pm \sigma_{0} \mu_{1}}{\sigma_{1}+\sigma_{0}}
\end{aligned}
$$

O sinal positivo desta expressão refere-se ao valor mínimo da probabilidade de erro, enquanto o sinal negativo refere-se a um ponto de máximo da mesma. Este ponto de máximo não é muito óbvio a partir de uma análise visual da curva de $P_{e}(D)$, e pode-se mostrar que este ponto é um máximo global da função. Como as assíntotas horizontais correspondem ao valor 0.5 , isto que quer dizer que existe um nível de decisão com o qual erra-se mais do que $50 \%$ das vezes. É importante notar que não é sempre que se pode desprezar o termo dentro do radical, pois sua contribuição para o posição do ponto de decisão ótimo pode ser muito importante. Nos gráficos das 


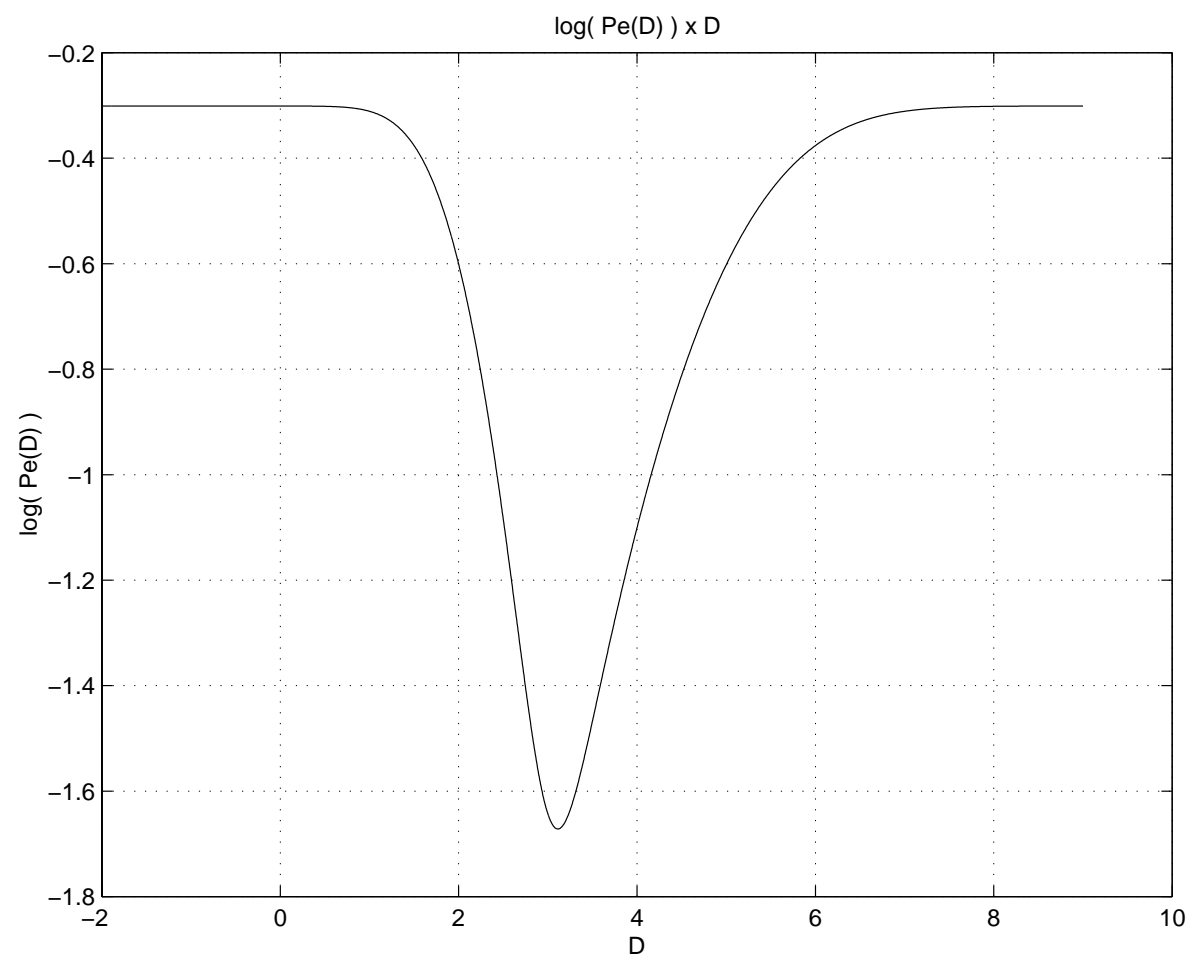

Figura 2.4: Gráfico da probabilidade de erro em função do nível de decisão. O ponto de máximo é de difícil visualização, e encontra-se à esquerda do valor mínimo

figuras 2.4, 2.5 e 2.6, $\mu_{0}=2, \sigma_{0}=0.5, \mu_{1}=5$ e $\sigma_{1}=1$. Se for calculado pela expressão completa, $D=3.1124$, enquanto que pela expressão simplificada, $D=3$. 


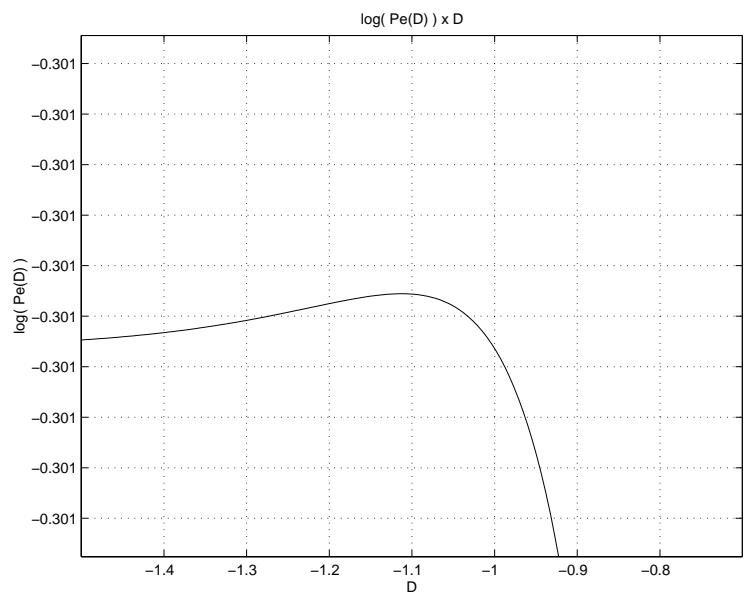

Figura 2.5: Gráfico da probabilidade de erro em função do nível de decisão, ressaltando o ponto de máximo

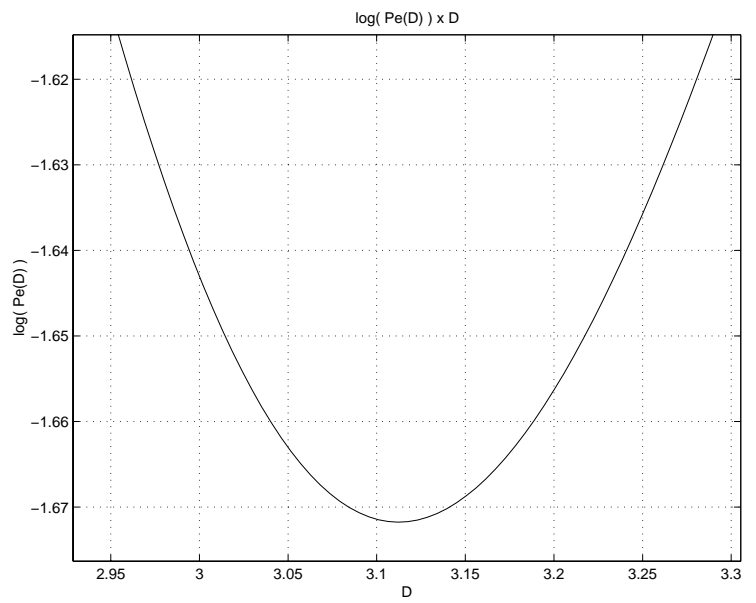

Figura 2.6: Gráfico da probabilidade de erro em função do nível de decisão, mostrando o ponto de mínimo calculado usando o ponto de decisão ótimo 


\subsection{Apêndice IV - Comparação entre os pontos de decisão}

O que se quer mostrar é que

$$
P_{e}^{s i m p l}\left(\mu_{0}, \mu_{1}, \sigma_{0}, \sigma_{1}\right)-P_{e, p r o x}^{s i m p l}\left(\mu_{0}, \mu_{1}, \sigma_{0}, \sigma_{1}\right)>0, \forall \mu_{0}, \mu_{1}, \sigma_{0}, \sigma_{1},
$$

ou seja

$$
\frac{1}{2}\left[Q\left(\frac{\mu_{1}-\mu_{0}}{2 \sigma_{0}}\right)+Q\left(\frac{\mu_{1}-\mu_{0}}{2 \sigma_{1}}\right)\right]-Q\left(\frac{\mu_{1}-\mu_{0}}{\sigma_{1}+\sigma_{0}}\right)>0 .
$$

Suponha então sem perda de generalidade que $\mu_{1}>\mu_{0} \quad$ e $\sigma_{1}>\sigma_{0}$. Seja então

$$
a=\frac{\mu_{1}-\mu_{0}}{2 \sigma_{0}}>0
$$

A expressão fica

$$
\frac{1}{2}\left[Q(a)+Q\left(a \frac{\sigma_{0}}{\sigma_{1}}\right)\right]-Q\left(a \frac{2 \sigma_{0}}{\sigma_{1}+\sigma_{0}}\right)>0 .
$$

Obviamente,

$$
\frac{\sigma_{0}}{\sigma_{1}}<\frac{2 \sigma_{0}}{\sigma_{1}+\sigma_{0}}<1
$$

o que implica em

$$
Q\left(a \frac{\sigma_{0}}{\sigma_{1}}\right)>Q\left(a \frac{2 \sigma_{0}}{\sigma_{1}+\sigma_{0}}\right)>Q(a)
$$

A validade da expressão (2.10.4) decorre então do fato que $\frac{d^{2} Q(x)}{d x^{2}}>0, \forall x>0$. A figura 2.7 ilustra a situação. 


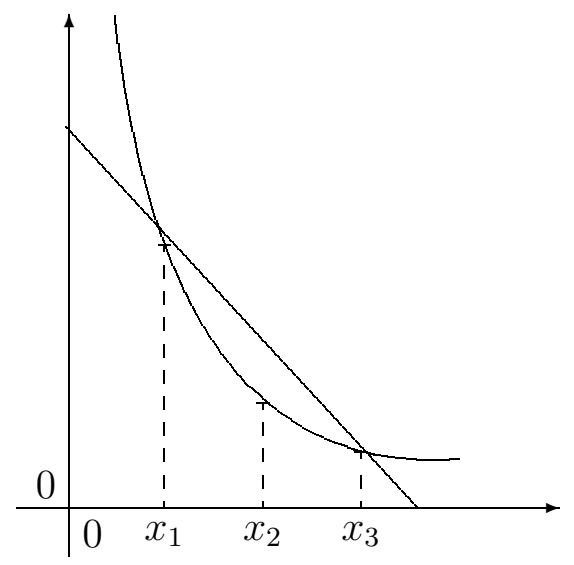

Figura 2.7: Comportamento da função Q(x) 


\section{Capítulo 3}

\section{Modelo Não-Gaussiano de Ruído Interferométrico}

\subsection{Introdução}

Neste capítulo é desenvolvido um modelo não gaussiano para o ruído interferométrico que utiliza a teoria das variáveis cíclicas desenvolvida no capítulo 1. Esperase que esse novo modelo seja melhor que o modelo gaussiano, devido à hipóteses mais realistas. Sem o uso de variáveis cíclicas não seria possível conseguir muitos dos resultados analíticos aqui obtidos.

Variáveis aleatórias cíclicas serão usadas para modelar ângulos, o que quer dizer, na notação do capítulo 1, que o grupo das variáveis aleatórias cíclicas angulares é $\mathbb{G}_{2 \pi}=\left(I_{[-\pi, 2 \pi]}, \oplus\right)$.

Ao longo do desenvolvimento deste capítulo, será necessário calcular a distribuição de probabilidade da soma de variáveis aleatórias independentes. É um resultado conhecido que esta distribuição de probabilidade é a convolução das distribuições de probabilidade das parcelas. Existe um problema de ordem prática associado ao cálculo 
de convoluções das funções aqui envolvidas. Para realizar uma convolução numericamente, é necessário fazer uma discretização, ou seja, deve-se escolher um intervalo para amostrar as funções regularmente. O problema é que como a densidade de probabilidade encontrada diverge nos dois pontos extremos do intervalo considerado ( figura 3.1 ), para se obter a precisão necessária é preciso utilizar uma discretização muito pequena próximo ao ponto de divergência. Com isso, o tempo para o cálculo da convolução aumenta, tornando o processo computacionalmente inviável. Tal problema foi resolvido utilizando-se transformadas de Fourier. Ao invés de se calcular numericamente as convoluções, calculam-se as transformadas de Fourier e fazem-se multiplicações ponto a ponto. No fim do processo, basta fazer uma FFT inversa e a distribuição desejada será obtida.

\subsection{Cálculo da Densidade de Probabilidade do Ruído Interferométrico}

Seguindo a mesma notação do capítulo anterior, seja $\vec{E}_{0}(t)=b_{0}(t) E_{0} e^{j(\omega t+\phi(t))} \vec{p}_{0}(t)$ a expressão do campo elétrico, onde $E_{0}=\sqrt{2 P_{s}}$ e $P_{s}$ é o valor RMS da potência ótica no detector, $b_{0}(t)$ é o bit de informação, que pode ser 0 ou 1, $\omega$ é a freqüência ótica, $\phi(t)$ é o ruído de fase e $p \overrightarrow{(t)}$ é o vetor de polarização. Fazendo $b_{0}\left(t-\tau_{i}\right)=b_{i}(t)$ e $\vec{p}_{0}\left(t-\tau_{i}\right)=\vec{p}_{i}(t)$, chega-se à seguinte expressão para o campo total:

$$
\vec{E}_{T}(t)=E_{0}\left[b_{0}(t) e^{j(\omega t+\phi(t))} \vec{p}_{0}(t)+\sum_{i=1}^{N} b_{i}(t) \sqrt{\alpha_{i}} e^{j\left(\omega\left(t-\tau_{i}\right)+\phi\left(t-\tau_{i}\right)\right)} \vec{p}_{i}(t)\right]
$$

No capítulo anterior, a contribuição dos termos de crosstalk secundário na intensidade da luz foi desprezada. Nos cálculos a seguir estes termos serão levados em 


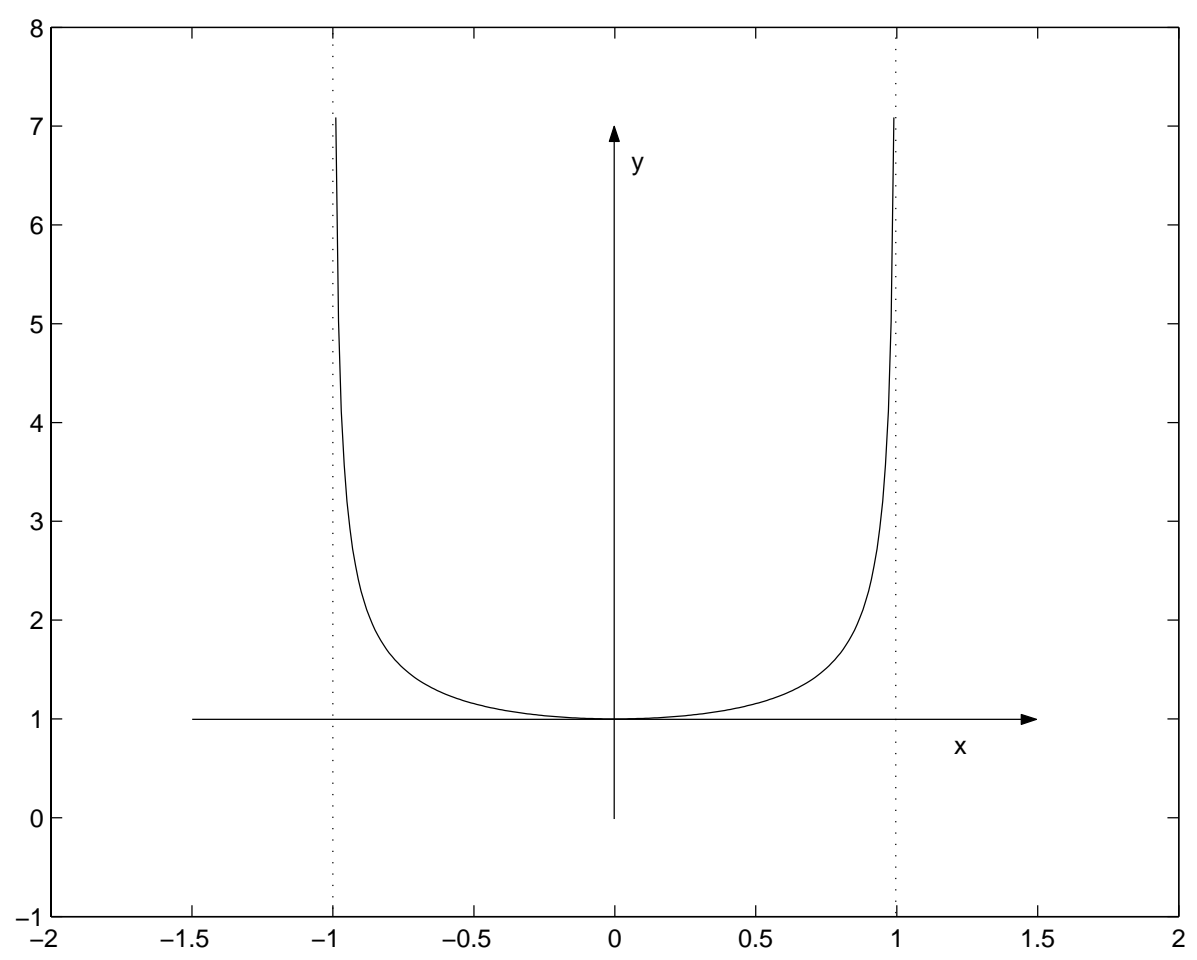

Figura 3.1: Gráfico da densidade de probabilidade do cosseno de uma v.a. uniforme consideração, de modo que quando se quiser expressões simplificadas, estes termos poderão ser desprezados. Assim, intensidade da luz vale

$$
\begin{aligned}
I(t)= & \left\|\overrightarrow{\mathbf{E}}_{T}(t)\right\|= \\
= & E_{0}^{2}\left[b_{0}^{2}(t)+\sum_{i=1}^{N} b_{i}^{2}(t) \alpha_{i}+2 b_{0}(t) \sum_{i=1}^{N} b_{i}(t) \sqrt{\alpha_{i}} \cos \left(\omega_{0} \tau_{i}+\phi(t)-\phi\left(t-\tau_{i}\right)\right) \cos \beta_{0} i\right. \\
& \left.+2 \sum_{k=1}^{i-1} \sqrt{\alpha_{i} \alpha_{k}} b_{k}(t) \cos \left(\omega_{0}\left(\tau_{i}-\tau_{k}\right)+\phi\left(t-\tau_{k}\right)-\phi\left(t-\tau_{i}\right)\right)\right]+n_{T}(t), \quad(3.2 .2)
\end{aligned}
$$

onde, mais uma vez, $\vec{p}_{i}(t) \cdot \vec{p}_{k}(t)=\cos \beta_{i k}$ e $n_{T}(t)$ é o ruído térmico. Então, usando o 
fato que $b_{i}^{2}(t)=b_{i}(t)$,

$$
\begin{aligned}
I(t)= & \\
= & E_{0}^{2}\left[b_{0}(t)+\sum_{i=1}^{N} b_{i}(t)\left\{\alpha_{i}+2 b_{0}(t) \sqrt{\alpha_{i}} \cos \left(\omega_{0} \tau_{i}+\phi(t)-\phi\left(t-\tau_{i}\right)\right) \cos \beta_{0} i\right.\right. \\
& \left.\left.+2 \sum_{k=1}^{i-1} \sqrt{\alpha_{i} \alpha_{k}} b_{k}(t) \cos \left(\omega_{0}\left(\tau_{i}-\tau_{k}\right)+\phi\left(t-\tau_{k}\right)-\phi\left(t-\tau_{i}\right)\right) \cos \beta_{i k}\right\}\right]+n_{T}(t) .
\end{aligned}
$$

Esquecendo a dependência em t e fazendo $X_{i 0}=\cos \left(\omega_{0} \tau_{i}+\phi(t)-\phi\left(t-\tau_{i}\right)\right)$ e $Y_{i 0}=\cos \beta_{i 0}$,

$$
I=E_{0}^{2}\left[b_{0}+\sum_{i=1}^{N} b_{i}\left\{\alpha_{i}+2 b_{0} \sqrt{\alpha_{i}} X_{i 0} Y_{i 0}+2 \sum_{k=1}^{i-1} \sqrt{\alpha_{i} \alpha_{k}} b_{k} X_{i k} Y_{i k}\right\}\right]+n_{T}
$$

Pode-se simplificar um pouco mais esta expressão fazendo $Z_{i k}=X_{i k} Y_{i k}$ :

$$
I=E_{0}^{2}\left[b_{0}+\sum_{i=1}^{N} b_{i}\left\{\alpha_{i}+2 b_{0} \sqrt{\alpha_{i}} Z_{i 0}+2 \sum_{k=1}^{i-1} \sqrt{\alpha_{i} \alpha_{k}} b_{k} Z_{i k}\right\}\right]+n_{T}
$$

A função densidade de probabilidade da variável $Z_{i k}$ vai depender das hipóteses assumidas.

Proposição 3.2.1. Seja $X=A \cos \alpha+B$, onde $A, B \in \mathbb{R}$ e $\alpha$ é uma variável aleatória cíclica angular uniforme. Então:

i) $p_{X}(x)=\frac{1}{\pi \sqrt{A^{2}-(x-B)^{2}}}, x \in[B-A, B+A]$

ii) $E[X]=B$

iii) $E\left[(X-B)^{2}\right]=A^{2} / 2$

iv) $\mathbf{F}\left[p_{X}(x)\right]=e^{-j \omega B} J_{0}(A \omega)$. 
Demonstração. Vide apêndice I.

Considere a função $\operatorname{Bat}(A, x-B)=\frac{1}{\pi \sqrt{A^{2}-(x-B)^{2}}}$. Se o erro de fase for uma variável aleatória cíclica angular uniforme, então conforme a proposição 3.2.1 $p_{A X_{i k}+B}(x)=$ $\operatorname{Bat}(A, x-B)$. Se as polarizações estiverem alinhadas, então $Y_{i k}=1$, de modo que $Z_{i k}=X_{i k}$, e portanto neste caso $p_{A Z_{i k}+B}(x)=p_{A X_{i k}+B}(x)=\operatorname{Bat}(A, x-B)$. Por outro lado, se o ângulo de polarização $\beta_{i k}$ for uma variável aleatória cíclica angular uniforme, então $p_{Y_{i k}}=\operatorname{Bat}(1, x)$, e será necessário a seguinte proposição para mostrar que $p_{A Z_{i k}+B}(x)=\operatorname{Bat}\left(\frac{A}{2}, z-B\right) \otimes \operatorname{Bat}\left(\frac{A}{2}, z\right)$.

Proposição 3.2.2. Seja $W=A X Y+B$, onde $X=\cos \alpha$ e $Y=\cos \beta, A, B \in \mathbb{R} e$ $\alpha$ e $\beta$ são variáveis aleatórias cíclicas angulares independentes. Então,

$$
p_{W}(w)=\operatorname{Bat}\left(\frac{A}{2}, w-B\right) \otimes \operatorname{Bat}\left(\frac{A}{2}, w\right)
$$

Demonstração. Escreva:

$$
\begin{aligned}
W & =A X Y+B \\
& =A \cos \alpha \cos \beta+B \\
& =\frac{A}{2}[\cos (\alpha+\beta)+\cos (\alpha-\beta)]+B
\end{aligned}
$$

Pelos corolários 1.9 .4 e 1.9.5, as variáveis aleatórias cíclicas angulares $\psi=\alpha+\beta$ e $\phi=\alpha-\beta$ são uniformes. Pelo corolário 1.9.9, $\psi$ e $\phi$ são independentes. Sejam $U=\frac{A}{2} \cos \psi$ e $V=\frac{A}{2} \cos \phi$. Deste modo, $W=U+V+B$. Pela proposição 3.2.1, $p_{U}(u)=\operatorname{Bat}\left(\frac{A}{2}, u\right)$ e $p_{V}(v)=\operatorname{Bat}\left(\frac{A}{2}, v\right)$. Como $\psi$ e $\phi$ são independentes, $U$ e $V$ também o são, e portanto

$$
p_{W}(w)=\operatorname{Bat}\left(\frac{A}{2}, w\right) \otimes \operatorname{Bat}\left(\frac{A}{2}, w\right) \otimes \delta(w-B)=\operatorname{Bat}\left(\frac{A}{2}, w-B\right) \otimes \operatorname{Bat}\left(\frac{A}{2}, w\right)
$$


Pode-se ainda, nas expressões da intensidade da luz, optar por desprezar ou não o termo de crosstalks secundários. Suponha inicialmente que os termos de crosstalk secundários sejam desprezados. Então a expressão da intensidade torna-se

$$
I=E_{0}^{2}\left\{b_{0}+\sum_{i=1}^{N} b_{i}\left[\alpha_{i}+2 b_{0} \sqrt{\alpha_{i}} Z_{i 0}\right]\right\}+n_{T} .
$$

A fim de calcular a função densidade de probabilidade da intensidade, utiliza-se a regra de Bayes particionando nos eventos $\left\{b_{0}=0\right\}$ e $\left\{b_{0}=1\right\}$ :

$$
\begin{aligned}
P[I] & =P\left[I \mid b_{0}=0\right] P\left[b_{0}=0\right]+P\left[I \mid b_{0}=1\right] P\left[b_{0}=1\right] \\
& =\frac{1}{2}\left(P\left[I \mid b_{0}=0\right]+P\left[I \mid b_{0}=1\right]\right)
\end{aligned}
$$

As expressões das intensidades condicionadas ao valor de $b_{0}$ tornam-se

$$
\begin{aligned}
& {\left[I \mid b_{0}=0\right]=E_{0}^{2}\left\{\sum_{i=1}^{N} b_{i} \alpha_{i}\right\}+n_{T}} \\
& \mathrm{e} \\
& {\left[I \mid b_{0}=1\right]=E_{0}^{2}\left\{1+\sum_{i=1}^{N} b_{i}\left[\alpha_{i}+2 \sqrt{\alpha_{i}} Z_{i 0}\right]\right\}+n_{T} .}
\end{aligned}
$$

Para achar a função densidade de probabilidade condicionada a $b_{0}=0$, basta supor que $b_{i}$ são as variáveis aleatórias de Bernoulli com $p=\frac{1}{2}$, independentes e identicamente distribuídas (iid), o que resulta numa soma de variáveis aleatórias independentes. Tem-se então que $p_{b_{i}}(x)=\frac{1}{2}[\delta(x)+\delta(x-1)]$, e assim pode-se escrever

$$
\begin{aligned}
p_{I \mid b_{0}=0}(x) & =p_{n_{T}+\sum_{i=1}^{N} E_{0}^{2} \alpha_{i} b_{i}}(x) \\
& =p_{n_{T}}(x) p_{\sum_{i=1}^{N} E_{0}^{2} \alpha_{i} b_{i}}(x) \\
& =p_{n_{T}}(x) \bigotimes_{i=1}^{N} p_{E_{0}^{2} \alpha_{i} b_{i}}(x) \\
& =p_{n_{T}}(x) \bigotimes_{i=1}^{N}\left\{\frac{1}{2}\left[\delta(x)-\delta\left(x-E_{0}^{2} \alpha_{i}\right)\right]\right\} .
\end{aligned}
$$


Para achar a função densidade de probabilidade condicionada a $b_{0}=1$, suponha que os $Z_{i 0}$ são iid e faça $W_{i 0}=E_{0}^{2}\left(\alpha_{i}+2 \sqrt{\alpha_{i}} Z_{i 0}\right)$. Então, $\sum_{i=1}^{N} b_{i} W_{i 0}$ é uma soma de variáveis aleatórias independentes, de modo que

$$
\begin{aligned}
p_{I \mid b_{0}=1}(x) & =p_{n_{T}+E_{0}^{2}+\sum_{i=1}^{N} b_{i} W_{i 0}}(x) \\
& =p_{n_{T}}(x) \otimes \delta\left(x-E_{0}^{2}\right) \otimes p_{\sum_{i=1}^{N} b_{i} W_{i 0}}(x) \\
& =p_{n_{T}}(x) \otimes \delta\left(x-E_{0}^{2}\right) \bigotimes_{i=1}^{N} p_{b_{i} W_{i 0}}(x) \\
& =p_{n_{T}}(x) \otimes \delta\left(x-E_{0}^{2}\right) \bigotimes_{i=1}^{N}\left\{\frac{1}{2}\left[\delta(x)+p_{W_{i 0}}(x)\right]\right\},
\end{aligned}
$$

onde o resultado da última passagem pode ser obtido utilizando-se mais uma vez a regra de Bayes e condicionando aos eventos $\left\{b_{i}=0\right\}$ e $\left\{b_{i}=1\right\}$.

Se as polarizações estiverem alinhadas, então $p_{W_{i 0}}(x)=\operatorname{Bat}\left(2 E_{0}^{2} \sqrt{\alpha_{i}}, x-E_{0}^{2} \alpha_{i}\right)$. Supondo então que o ruído térmico é gaussiano de média nula e variância $\sigma^{2}$, é possível escrever as transformadas de Fourier das funções densidade de probabilidade condicionais como

$$
\begin{aligned}
\mathbf{F}\left[p_{I \mid b_{0}=0}(x)\right] & =\mathbf{E}_{I \mid b_{0}=0}\left[e^{-j \omega x}\right]=e^{-\frac{\omega^{2} \sigma^{2}}{2}} \prod_{i=1}^{N}\left[\frac{1}{2}\left(1+e^{-j \omega E_{0}^{2} \alpha_{i}}\right)\right] \\
& \mathrm{e}\left[p_{I \mid b_{0}=1}(x)\right]=\mathbf{E}_{I \mid b_{0}=1}\left[e^{-j \omega x}\right] \\
& =e^{-\frac{\omega^{2} \sigma^{2}}{2}-j \omega E_{0}^{2}} \prod_{i=1}^{N}\left[\frac{1}{2}\left(1+e^{-j \omega E_{0}^{2} \alpha_{i}} J_{0}\left(2 E_{0}^{2} \sqrt{\alpha_{i}} \omega\right)\right)\right]
\end{aligned}
$$

Onde $J_{0}$ é a função de Bessel de ordem 0 de primeira espécie. Faça agora $\alpha_{i}=\alpha, \forall i$, o que simplifica as expressões anteriores para

$$
\mathbf{F}\left[p_{I \mid b_{0}=0}(x)\right]=e^{-\frac{\omega^{2} \sigma^{2}}{2}}\left[\frac{1}{2}\left(1+e^{-j \omega E_{0}^{2} \alpha}\right)\right]^{N}
$$




$$
\mathbf{F}\left[p_{I \mid b_{0}=1}(x)\right]=e^{-\frac{\omega^{2} \sigma^{2}}{2}-j \omega E_{0}^{2}}\left[\frac{1}{2}\left(1+e^{-j \omega E_{0}^{2} \alpha} J_{0}\left(2 E_{0}^{2} \sqrt{\alpha} \omega\right)\right)\right]^{N}
$$

Se por outro lado, as polarizações não estiverem alinhadas e o ângulo de polarização for uma variável aleatória uniforme, então

$$
p_{W_{i 0}}(x)=\delta\left(x-E_{0}^{2} \alpha_{i}\right) \otimes \operatorname{Bat}\left(E_{0}^{2} \sqrt{\alpha_{i}}, x\right) \otimes \operatorname{Bat}\left(E_{0}^{2} \sqrt{\alpha_{i}}, x\right)
$$

Isto possibilita que as transformadas de Fourier das funções densidade de probabilidade condicionais sejam agora escritas como

$$
\begin{aligned}
\mathbf{F}\left[p_{I \mid b_{0}=0}(x)\right] & =\mathbf{E}_{I \mid b_{0}=0}\left[e^{-j \omega x}\right]=e^{-\frac{\omega^{2} \sigma^{2}}{2}} \prod_{i=1}^{N}\left[\frac{1}{2}\left(1+e^{-j \omega E_{0}^{2} \alpha_{i}}\right)\right] \\
& \mathrm{e}\left[p_{I \mid b_{0}=1}(x)\right]=\mathbf{E}_{I \mid b_{0}=1}\left[e^{-j \omega x}\right] \\
& =e^{-\frac{\omega^{2} \sigma^{2}}{2}-j \omega E_{0}^{2}} \prod_{i=1}^{N}\left[\frac{1}{2}\left(1+e^{-j \omega E_{0}^{2} \alpha_{i}} J_{0}^{2}\left(E_{0}^{2} \sqrt{\alpha_{i}} \omega\right)\right)\right] .
\end{aligned}
$$

Fazendo $\alpha_{i}=\alpha, \forall i$, obtém-se

$$
\begin{array}{rl}
\mathbf{F}\left[p_{I \mid b_{0}=0}(x)\right] & =e^{-\frac{\omega^{2} \sigma^{2}}{2}}\left[\frac{1}{2}\left(1+e^{-j \omega E_{0}^{2} \alpha}\right)\right]^{N} \\
\mathrm{e} & \mathbf{F}\left[p_{I \mid b_{0}=1}(x)\right]=e^{-\frac{\omega^{2} \sigma^{2}}{2}-j \omega E_{0}^{2}}\left[\frac{1}{2}\left(1+e^{-j \omega E_{0}^{2} \alpha} J_{0}^{2}\left(E_{0}^{2} \sqrt{\alpha} \omega\right)\right)\right]^{N} .
\end{array}
$$

Suponha agora que os crosstalks secundários não sejam desprezados. Então a expressão da intensidade da luz é

$$
I=E_{0}^{2}\left[b_{0}+\sum_{i=1}^{N} b_{i}\left(\alpha_{i}+2 b_{0} \sqrt{\alpha_{i}} Z_{i 0}+2 \sum_{k=1}^{i-1} \sqrt{\alpha_{i} \alpha_{k}} b_{k} Z_{i k}\right)\right]+n_{T}
$$

portanto

$$
i=0 \Rightarrow E_{0}^{2} b_{0}
$$




$$
\begin{aligned}
& i=1 \Rightarrow E_{0}^{2} b_{1}\left[\alpha_{1}+2 b_{0} \sqrt{\alpha_{1}} Z_{10}+0\right] \\
& i=2 \Rightarrow E_{0}^{2} b_{2}\left[\alpha_{2}+2 b_{0} \sqrt{\alpha_{2}} Z_{20}+2 \sqrt{\alpha_{2} \alpha_{1}} b_{1} Z_{21}\right] \\
& i=3 \Rightarrow E_{0}^{2} b_{3}\left[\alpha_{3}+2 b_{0} \sqrt{\alpha_{3}} Z_{30}+2 \sqrt{\alpha_{3} \alpha_{1}} b_{1} Z_{31}+2 \sqrt{\alpha_{3} \alpha_{2}} b_{2} Z_{32}\right] .
\end{aligned}
$$

Pode-se observar a partir do quadro acima que a soma dos termos de $i$ variando de zero a $N$ não é uma soma de termos independentes. A fim de calcular a função densidade de probabilidade da intensidade luminosa, basta empregar a regra de Bayes utilizando como partição todas as combinações possíveis de valores de $b_{i}$. Cada evento pode ser representado por um vetor $\vec{b} \in B^{N}=\{0,1\}^{N}$, onde $\vec{b}=\left(b_{1}, \ldots, b_{N}\right)$. Como as funções densidade de probabilidade só dependem do número de bits diferentes de zero, e não do conjunto particular de valores, pode-se agrupar os eventos da seguinte forma: seja $n=\sum_{i=1}^{N} b_{i}$. Então os conjuntos $U^{n}=\left\{b^{n, j} \in B^{N} \mid \sum_{i=1}^{N} b_{i}^{n, j}=n\right\}$ formam uma partição do espaço $B^{N}$. Cada conjunto $U^{n}$ possui $\left(\begin{array}{l}N \\ n\end{array}\right)$ elementos, e assim pode-se escrever

$$
p_{I \mid b_{0}}(x)=\sum_{n=0}^{N} \sum_{j=1}^{\left(\begin{array}{c}
N \\
n
\end{array}\right)} p_{I \mid b_{0}, b^{n, j}}(x) \cdot P\left[b^{n, j}\right]
$$

$\operatorname{mas} P\left[b^{n, j}\right]=2^{-N}$ e $p_{I \mid b_{0}, b^{n, j 1}}(x)=p_{I \mid b_{0}, b^{n, j 2}}(x), \forall j_{1}, j_{2}$. Deste modo,

$$
p_{I \mid b_{0}}(x)=2^{-N} \sum_{n=0}^{N}\left(\begin{array}{c}
N \\
n
\end{array}\right) p_{I \mid b_{0}, b^{n, j}}(x) .
$$

A expressão da intensidade luminosa condicionada ao evento $\left\{b^{n, j}\right\}$ pode ser escrita como

$$
\left[I \mid b_{0}, b^{n, j}\right]=n_{T}+E_{0}^{2}\left\{b_{0}+\sum_{i=1}^{N} b_{i}^{n, j}\left[\alpha_{i}+2 b_{0} \sqrt{\alpha_{i}} Z_{i 0}+\sum_{k=1}^{i-1} 2 \sqrt{\alpha_{i} \alpha_{k}} b_{k}^{n, j} Z_{i k}\right]\right\},
$$

onde pode-se notar que o primeiro somatório em $i$ possui $n$ termos não nulos, enquanto o duplo somatório em $i$ e $k$ possui $\left(\begin{array}{l}n \\ 2\end{array}\right)$ termos não nulos. Fazendo $W_{i 0}=$ 
$E_{0}^{2}\left(\alpha_{i}+2 \sqrt{\alpha_{i}} Z_{i 0}\right)$ e $V_{i k}=2 E_{0}^{2} \sqrt{\alpha_{i} \alpha_{k}} Z_{i k}$, obtém-se

$$
\begin{aligned}
{\left[I \mid b_{0}=0, b^{n, j}\right] } & =n_{T}+\sum_{i=1}^{N} b_{i}^{n, j}\left[E_{0}^{2} \alpha_{i}+\sum_{k=1}^{i-1} b_{k}^{n, j} V_{i k}\right] \\
\mathrm{e} & \\
{\left[I \mid b_{0}=1, b^{n, j}\right] } & =n_{T}+E_{0}^{2}+\sum_{i=1}^{N} b_{i}^{n, j}\left[W_{i 0}+\sum_{k=1}^{i-1} b_{k}^{n, j} V_{i k}\right] .
\end{aligned}
$$

As somas resultantes são de variáveis aleatórias independentes, e portanto

$$
\begin{aligned}
p_{\left[I \mid b_{0}=0, b^{n, j}\right]}(x) & =p_{n_{T}+\sum_{i=1}^{N} b_{i}^{n, j}\left[E_{0}^{2} \alpha_{i}+\sum_{k=1}^{i-1} b_{k}^{n, j} V_{i k}\right]} \\
& =p_{n_{T}}(x) \otimes p_{\sum_{i=1}^{N} b_{i}^{n, j} E_{0}^{2} \alpha_{i}}(x) \otimes p_{\sum_{i=1}^{N} b_{i}^{n, j} \sum_{k=1}^{i-1} b_{k}^{n, j} V_{i k}}(x) \\
& \mathrm{e} \\
p_{\left[I \mid b_{0}=1, b^{n, j}\right]}(x) & =p_{n_{T}+E_{0}^{2}+\sum_{i=1}^{N} b_{i}^{n, j}\left[W_{i 0}+\sum_{k=1}^{i-1} b_{k}^{n, j} V_{i k}\right]}(x) \\
& =p_{n_{T}}(x) \otimes \delta\left(x-E_{0}^{2}\right) \otimes p_{\sum_{i=1}^{N} b_{i}^{n, j} W_{i 0}}(x) \otimes p_{\sum_{i=1}^{N} b_{i}^{n, j} \sum_{k=1}^{i-1} b_{k}^{n, j} V_{i k}}(x) .
\end{aligned}
$$

Fazendo $\alpha_{i}=\alpha, \forall i$, tem-se que $p_{V_{i k}}(x)=p_{V_{l m}}(x)=p_{V}(x), \forall i, k, l, m$, tornando-se possível simplificar as expressões anteriores e escrever

$$
\begin{gathered}
p_{\left[I \mid b_{0}=0, b^{n, j}\right]}(x)=p_{n_{T}}(x) \bigotimes_{i=1}^{n} \delta\left(x-E_{0}^{2} \alpha\right) \bigotimes_{i=1}^{\left(\begin{array}{c}
n \\
2
\end{array}\right)} p_{V}(x) \\
\text { e } \\
p_{\left[I \mid b_{0}=1, b^{n, j}\right]}(x)=p_{n_{T}}(x) \otimes \delta\left(x-E_{0}^{2}\right) \bigotimes_{i=1}^{n} p_{W}(x) \bigotimes_{i=1}^{\left(\begin{array}{c}
n \\
2
\end{array}\right)} p_{V}(x) .
\end{gathered}
$$

As expressões completas das densidades de probabilidade condicionais ficam então

$$
\begin{aligned}
p_{I \mid b_{0}=0}(x) & =2^{-N} \sum_{n=0}^{N}\left(\begin{array}{l}
N \\
n
\end{array}\right) p_{n_{T}}(x) \bigotimes_{i=1}^{n} \delta\left(x-E_{0}^{2} \alpha\right) \bigotimes_{i=1}^{\left(\begin{array}{c}
n \\
2
\end{array}\right)} p_{V}(x) \\
& =2^{-N} p_{n_{T}}(x) \otimes \sum_{n=0}^{N}\left(\begin{array}{l}
N \\
n
\end{array}\right) \bigotimes_{i=1}^{n} \delta\left(x-E_{0}^{2} \alpha\right) \bigotimes_{i=1}^{\left(\begin{array}{c}
n \\
2
\end{array}\right)} p_{V}(x)
\end{aligned}
$$




$$
\begin{aligned}
p_{I \mid b_{0}=1}(x) & =2^{-N} \sum_{n=0}^{N}\left(\begin{array}{l}
N \\
n
\end{array}\right) p_{n_{T}}(x) \otimes \delta\left(x-E_{0}^{2}\right) \bigotimes_{i=1}^{n} p_{W}(x) \bigotimes_{i=1}^{\left(\begin{array}{c}
n \\
2
\end{array}\right)} p_{V}(x) \\
& =2^{-N} p_{n_{T}}(x) \otimes \delta\left(x-E_{0}^{2}\right) \otimes \sum_{n=0}^{N}\left(\begin{array}{c}
N \\
n
\end{array}\right) \bigotimes_{i=1}^{n} p_{W}(x) \bigotimes_{i=1}^{n} p_{V}(x) .
\end{aligned}
$$

As transformadas de Fourier escrevem-se

$$
\begin{array}{rl}
\mathbf{F}\left[p_{I \mid b_{0}=0}(x)\right] & =e^{\frac{-\omega^{2} \sigma^{2}}{2}} 2^{-N} \sum_{n=0}^{N}\left(\begin{array}{c}
N \\
n
\end{array}\right) e^{-j \omega E_{0}^{2} n \alpha} F^{\left(\begin{array}{c}
n \\
2
\end{array}\right)}\left[p_{V}(x)\right] \\
\mathrm{e} & \mathbf{F}\left[p_{I \mid b_{0}=1}(x)\right]=e^{\frac{-\omega^{2} \sigma^{2}}{2}-j \omega E_{0}^{2}} 2^{-N} \sum_{n=0}^{N}\left(\begin{array}{c}
N \\
n
\end{array}\right) F^{n}\left[p_{W}(x)\right] F^{\left(\begin{array}{c}
n \\
2
\end{array}\right)}\left[p_{V}(x)\right] .
\end{array}
$$

Se as polarizações estiverem alinhadas, então $p_{W}(x)=\operatorname{Bat}\left(2 E_{0}^{2} \sqrt{\alpha}, x-E_{0}^{2} \alpha\right)$ e $p_{V}(x)=\operatorname{Bat}\left(2 E_{0}^{2} \alpha, x\right)$. Isto possibilita escrever as transformadas de Fourier das funções densidade de probabilidade condicionais como

$$
\begin{gathered}
\mathbf{F}\left[p_{I \mid b_{0}=0}(x)\right]=e^{\frac{-\omega^{2} \sigma^{2}}{2}} 2^{-N} \sum_{n=0}^{N}\left(\begin{array}{c}
N \\
n
\end{array}\right) e^{-j \omega E_{0}^{2} n \alpha} J_{0}^{\frac{n(n-1)}{2}}\left(2 E_{0}^{2} \alpha \omega\right) \\
\mathrm{e} \\
\mathbf{F}\left[p_{I \mid b_{0}=1}(x)\right]=e^{\frac{-\omega^{2} \sigma^{2}}{2}-j \omega E_{0}^{2}} 2^{-N} \sum_{n=0}^{N}\left(\begin{array}{c}
N \\
n
\end{array}\right) e^{-j \omega E_{0}^{2} n \alpha} J_{0}^{n}\left(2 E_{0}^{2} \sqrt{\alpha} \omega\right) J_{0}^{\frac{n(n-1)}{2}}\left(2 E_{0}^{2} \alpha \omega\right) .
\end{gathered}
$$

Se as polarizações não estiverem alinhadas, e o ângulo de polarização for uma variável aleatória uniforme, então

$$
p_{W}(x)=\delta\left(x-E_{0}^{2} \alpha\right) \otimes \operatorname{Bat}\left(E_{0}^{2} \sqrt{\alpha}, x\right) \otimes \operatorname{Bat}\left(E_{0}^{2} \sqrt{\alpha}, x\right)
$$




$$
p_{V}(x)=\operatorname{Bat}\left(E_{0}^{2} \alpha, x\right) \otimes \operatorname{Bat}\left(E_{0}^{2} \alpha, x\right)
$$

Isto possibilita escrever as transformadas de Fourier das funções densidade de probabilidade condicionais como

$$
\begin{gathered}
\mathbf{F}\left[p_{I \mid b_{0}=0}(x)\right]=e^{\frac{-\omega^{2} \sigma^{2}}{2}} 2^{-N} \sum_{n=0}^{N}\left(\begin{array}{c}
N \\
n
\end{array}\right) e^{-j \omega E_{0}^{2} n \alpha} J_{0}^{n(n-1)}\left(E_{0}^{2} \alpha \omega\right) \\
\mathrm{e} \\
\mathbf{F}\left[p_{I \mid b_{0}=1}(x)\right]=e^{\frac{-\omega^{2} \sigma^{2}}{2}-j \omega E_{0}^{2}} 2^{-N} \sum_{n=0}^{N}\left(\begin{array}{c}
N \\
n
\end{array}\right) e^{-j \omega E_{0}^{2} n \alpha} J_{0}^{2 n}\left(E_{0}^{2} \sqrt{\alpha} \omega\right) J_{0}^{n(n-1)}\left(E_{0}^{2} \alpha \omega\right) .
\end{gathered}
$$

\subsection{Expressões das Transformadas de Fourier para o caso Gaussiano}

É comum na literatura se utilizar um modelo onde a distribuição de probabilidade de $A \cos (\alpha)+B$ é aproximada por uma gaussiana com média $B$ e variância $A^{2} / 2$ $[14,11,16,15,17,26,6,31,5,45,8,28,21,41]$. A suposta utilidade desta aproximação é que se espera que para um número suficientemente grande de crosstalks a soma resultante tenha uma distribuição gaussiana, de acordo com o teorema do limite central. Além disso, a distribuição gaussiana é muito conveniente para cálculos analíticos.

As expressões análogas à 3.2.14 e 3.2.15, 3.2.19 e 3.2.20, 3.2.35 e 3.2.36, 3.2.39 e 3.2.40 para o caso gaussiano podem ser obtidas repetindo-se as mesmas contas e utilizando-se uma distribuição gaussiana. Desprezando o crosstalk secundário e com polarizações alinhadas obtém-se:

$$
\mathbf{F}\left[p_{I \mid b_{0}=0}(x)\right]=e^{-\frac{\omega^{2} \sigma^{2}}{2}}\left[\frac{1}{2}\left(1+e^{-j \omega E_{0}^{2} \alpha}\right)\right]^{N}
$$




$$
\mathbf{F}\left[p_{I \mid b_{0}=1}(x)\right]=e^{-\frac{\omega^{2} \sigma^{2}}{2}-j \omega E_{0}^{2}}\left[\frac{1}{2}\left(1+e^{-\omega^{2} E_{0}^{4} \alpha-j \omega E_{0}^{2} \alpha}\right)\right]^{N} .
$$

Desprezando o crosstalk secundário e com polarizações não-alinhadas obtém-se:

$$
\begin{aligned}
\mathbf{F}\left[p_{I \mid b_{0}=0}(x)\right] & =e^{-\frac{\omega^{2} \sigma^{2}}{2}}\left[\frac{1}{2}\left(1+e^{-j \omega E_{0}^{2} \alpha}\right)\right]^{N} \\
\mathrm{e} & \\
\mathbf{F}\left[p_{I \mid b_{0}=1}(x)\right] & =e^{\frac{-\omega^{2} \sigma^{2}}{2}-j \omega E_{0}^{2}}\left[\frac{1}{2}\left(1+e^{-\frac{\omega^{2}}{2} E_{0}^{4} \alpha-j \omega E_{0}^{2} \alpha}\right)\right]^{N} .
\end{aligned}
$$

Levando em conta o crosstalk secundário e com polarizações alinhadas obtém-se:

$$
\begin{gathered}
\mathbf{F}\left[p_{I \mid b_{0}=0}(x)\right]=e^{\frac{-\omega^{2} \sigma^{2}}{2}} 2^{-N} \sum_{n=0}^{N}\left(\begin{array}{c}
N \\
n
\end{array}\right) e^{-\frac{\omega^{2}}{2} E_{0}^{4} \alpha^{2} n(n-1)-j \omega E_{0}^{2} n \alpha} \\
\mathrm{e} \\
\mathbf{F}\left[p_{I \mid b_{0}=1}(x)\right]=e^{\frac{-\omega^{2} \sigma^{2}}{2}-j \omega E_{0}^{2}} 2^{-N} \sum_{n=0}^{N}\left(\begin{array}{c}
N \\
n
\end{array}\right) e^{-\frac{\omega^{2}}{2} E_{0}^{4} \alpha n[2+(n-1) \alpha]-j \omega E_{0}^{2} n \alpha} .
\end{gathered}
$$

Levando em conta o crosstalk secundário e com polarizações não-alinhadas obtémse:

$$
\begin{gathered}
\mathbf{F}\left[p_{I \mid b_{0}=0}(x)\right]=e^{\frac{-\omega^{2} \sigma^{2}}{2}} 2^{-N} \sum_{n=0}^{N}\left(\begin{array}{c}
N \\
n
\end{array}\right) e^{-\frac{\omega^{2}}{4} E_{0}^{4} \alpha^{2} n(n-1)-j \omega E_{0}^{2} n \alpha} \\
\mathrm{e} \\
\mathbf{F}\left[p_{I \mid b_{0}=1}(x)\right]=e^{\frac{-\omega^{2} \sigma^{2}}{2}-j \omega E_{0}^{2}} 2^{-N} \sum_{n=0}^{N}\left(\begin{array}{c}
N \\
n
\end{array}\right) e^{-\frac{\omega^{2}}{4} E_{0}^{4} \alpha n[2+(n-1) \alpha]-j \omega E_{0}^{2} n \alpha} .
\end{gathered}
$$




\subsection{Programa para o Cálculo das Penalidades de Potência}

De posse das expressões das transformadas de Fourier tanto no caso Gaussiano quanto no caso não-Gaussiano, foi possível escrever um programa de computador para realizar o cálculo das funções densidade de probabilidade e posteriormente as penalidades de potência em ambos os casos e fazer as comparações. Devido à quantidade de cálculos envolvida, o programa foi rodado apenas para os casos em que o crosstalk secundário é desprezado, com ou sem polarizações alinhadas. Os resultados podem ser vistos a seguir, onde os modelos são comparados também com o modelo simplificado (equação 2.3.1). O nível de decisão utilizado foi o da equação 2.4.5.

As curvas correspondem a um número de crosstalks igual a $2^{N-1}$, isto é, vão desde 1 até 64 crosstalks em potências de 2. Note como nas figuras 3.4 e 3.7 os modelos gaussiano e não-gaussiano tendem a se aproximar quando o número de crosstalks cresce. Note também que, conforme esperado, quando se modela o efeito de polarização aleatória, a penalidade de potência é menor para um mesmo nível de crosstalk. 


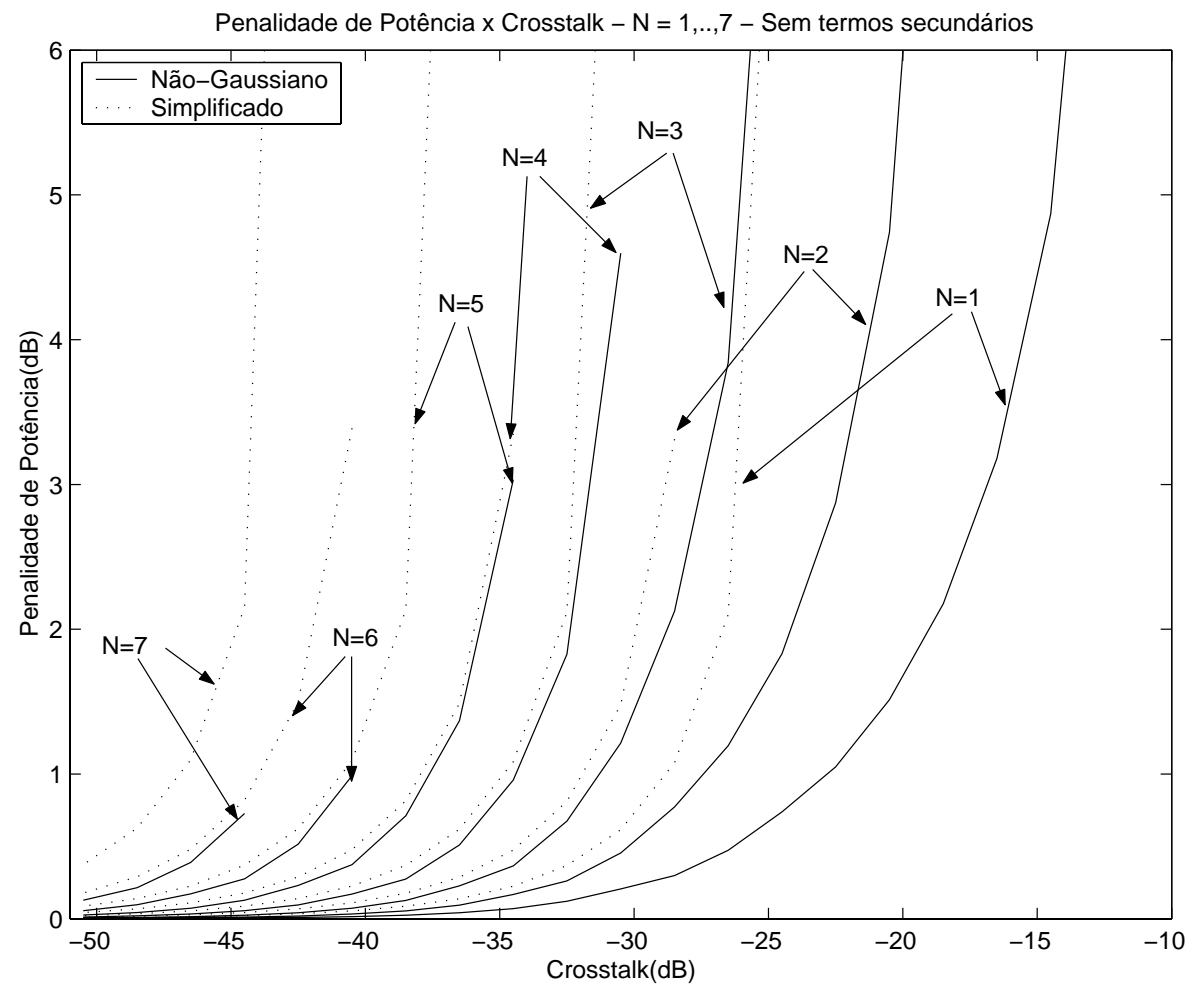

Figura 3.2: Modelo Não-Gaussiano x Simplificado - Polarizações Alinhadas 


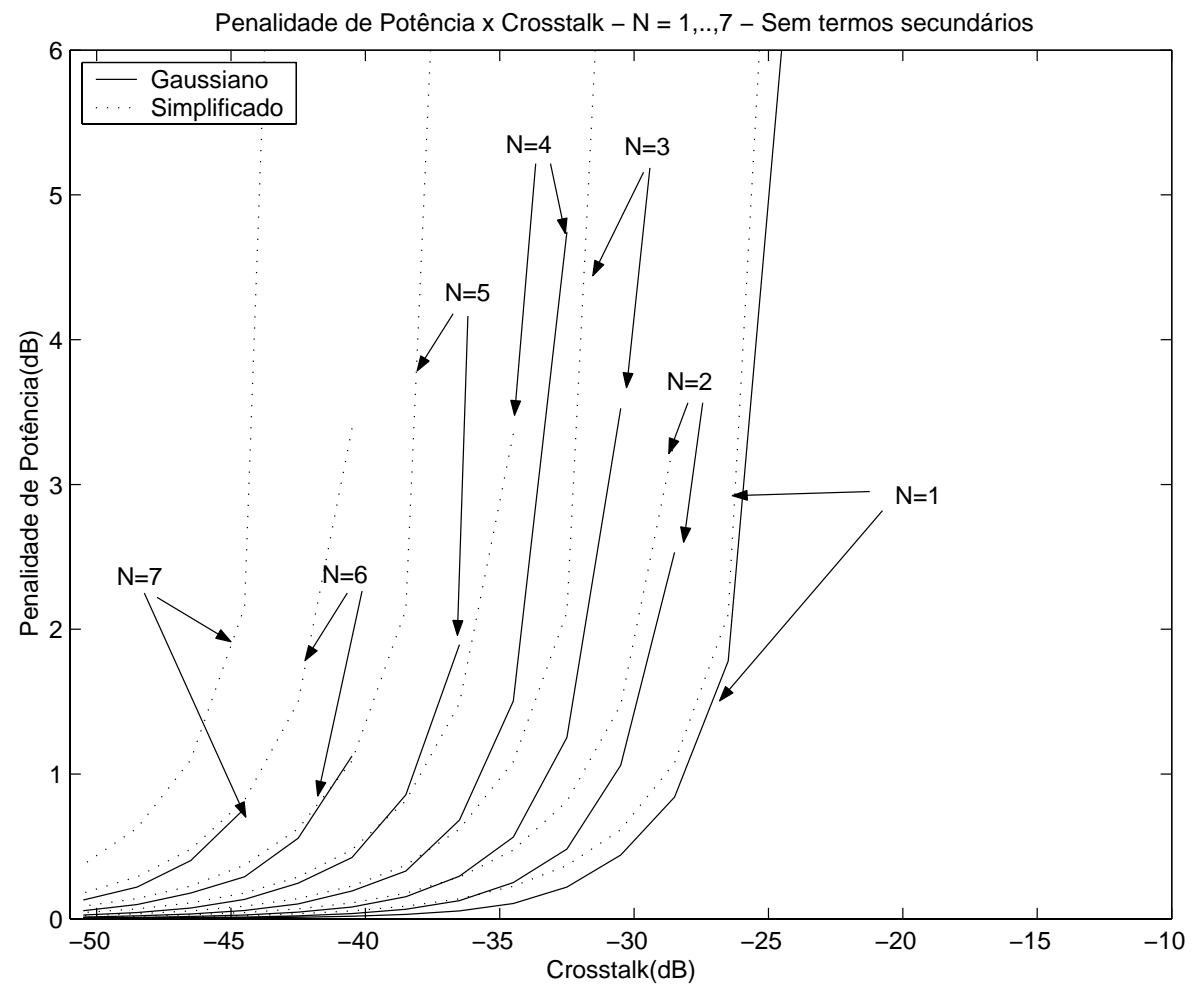

Figura 3.3: Modelo Gaussiano x Simplificado - Polarizações Alinhadas 


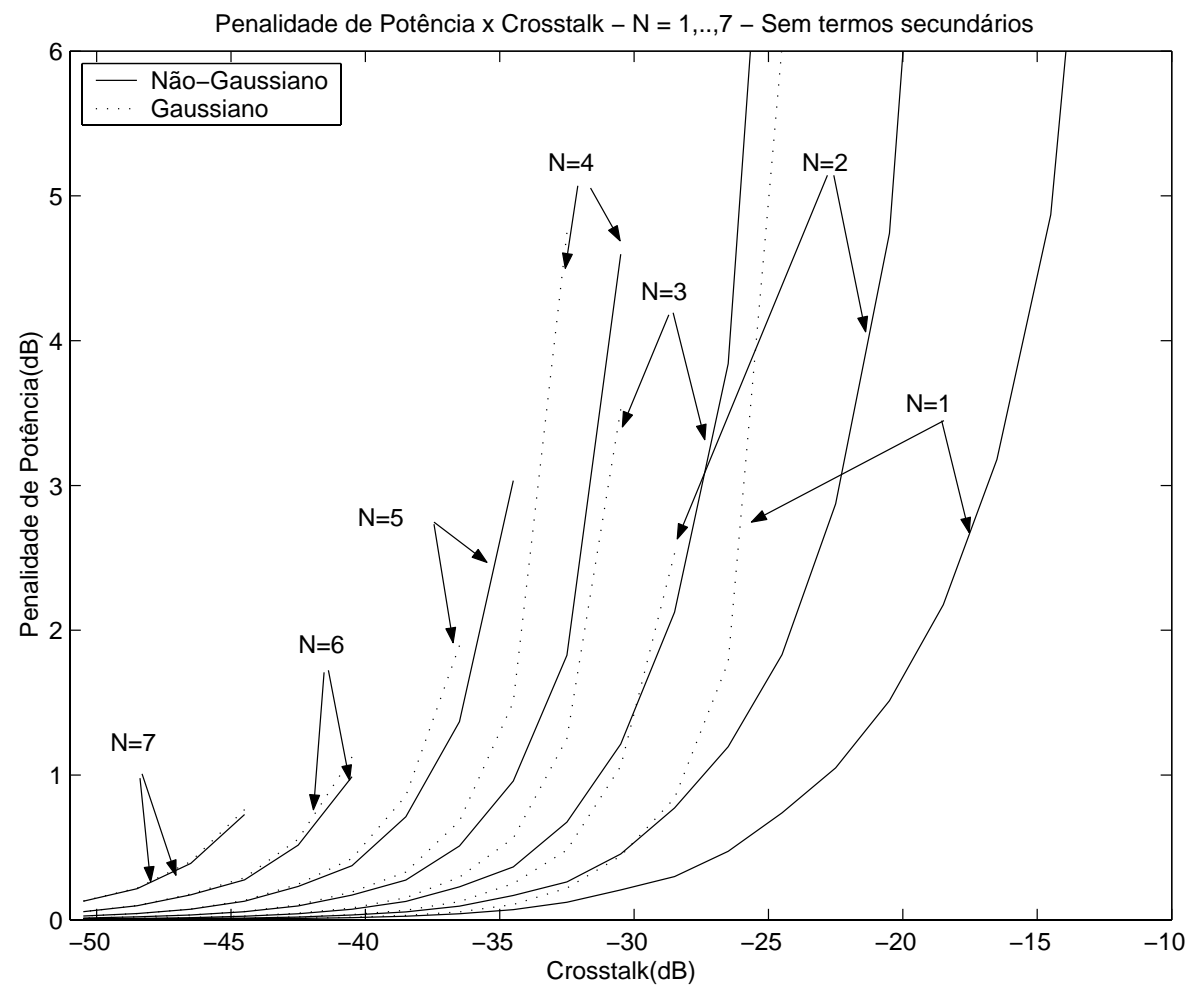

Figura 3.4: Modelo Gaussiano x Não-Gaussiano - Polarizações Alinhadas 


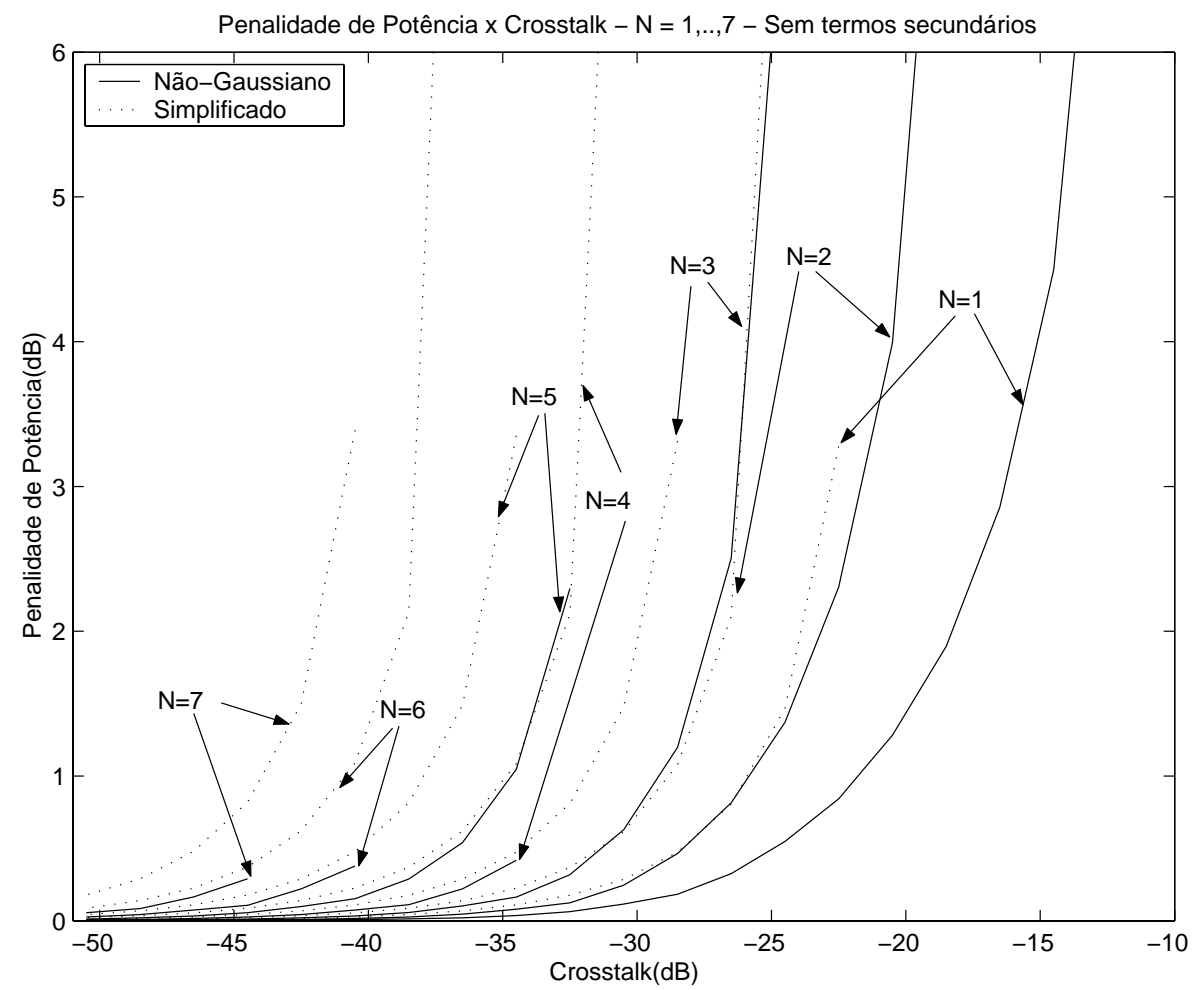

Figura 3.5: Modelo Não-Gaussiano x Simplificado - Polarizações Não-Alinhadas 


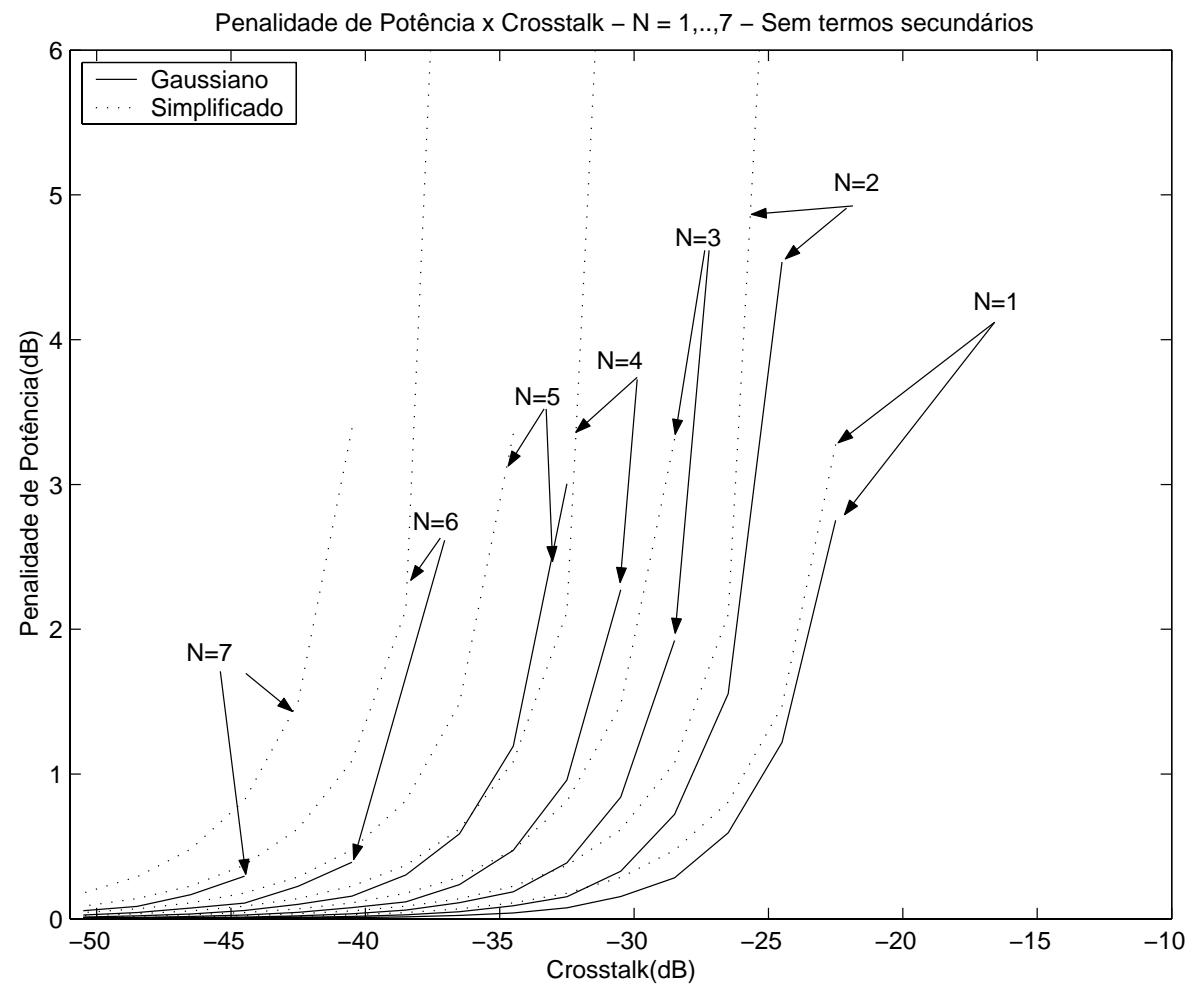

Figura 3.6: Modelo Gaussiano x Simplificado - Polarizações Não-Alinhadas 


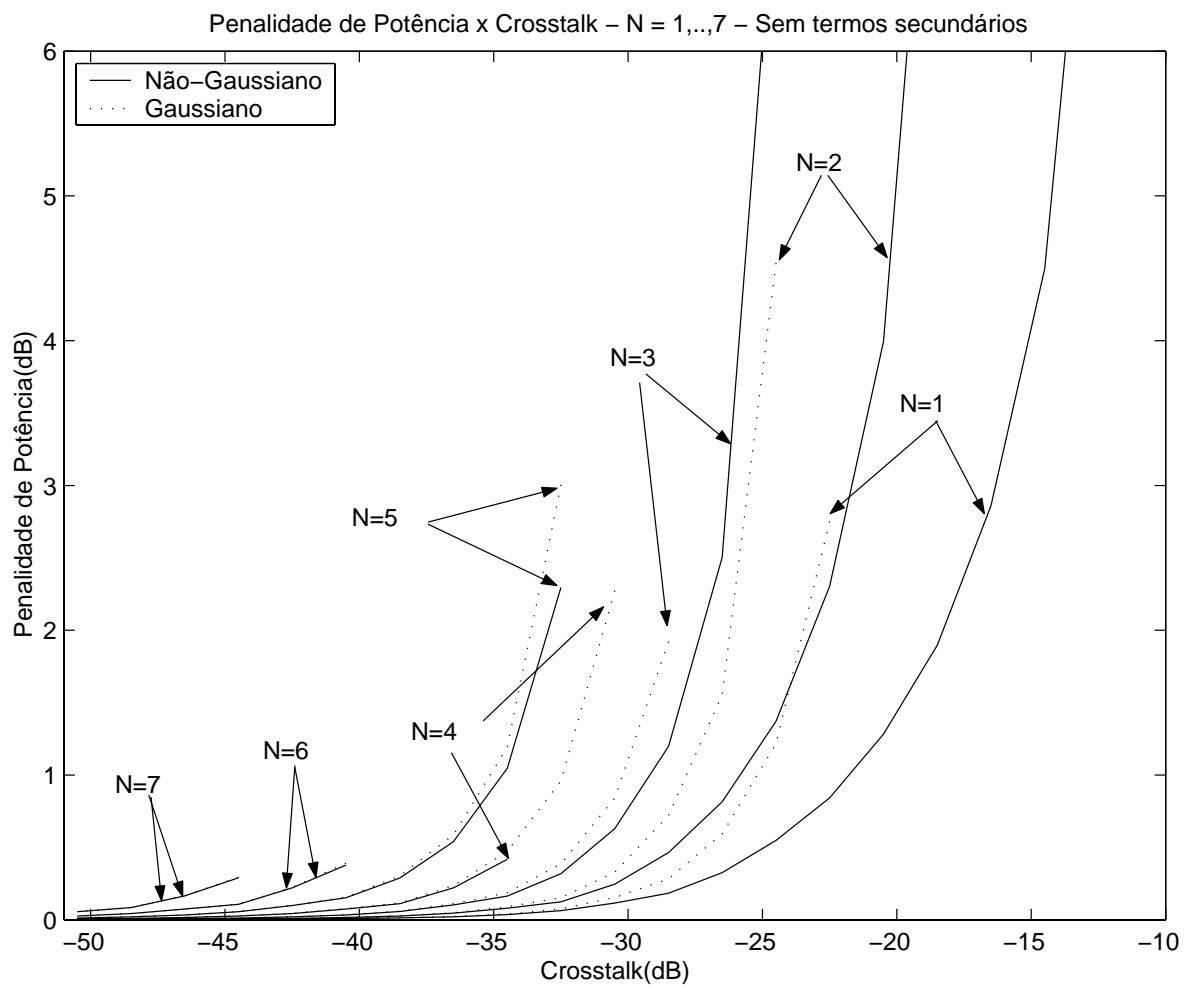

Figura 3.7: Modelo Gaussiano x Não-Gaussiano - Polarizações Não-Alinhadas

É interessante comparar os resultados obtidos para o caso não-gaussiano com os obtidos em [31]. Neste trabalho obtém-se um limitante superior para a probabilidade de erro através do uso de um limitante de Chernoff modificado [33]. Esse limitante é utilizado no cálculo das penalidades de potência e seus resultados estão de acordo com os aqui obtidos na figura 3.4. 


\subsection{Apêndice I - Expressões Utilizadas}

Seja $X \sim$ Uniforme $([-\pi, \pi])$ e $Y=A \cos (X)+B$. Então

$$
\begin{aligned}
& P[Y<=y]=P\left[X<=-\arccos \left(\frac{Y-B}{A}\right)\right]+P\left[X>=\arccos \left(\frac{Y-B}{A}\right)\right] \\
& =\int_{-\pi}^{-\arccos \left(\frac{Y-B}{A}\right)} \frac{1}{2 \pi} d x+\int_{\arccos \left(\frac{Y-B}{A}\right)}^{\pi} \frac{1}{2 \pi} d x \\
& =\left|\begin{array}{c}
y=A \cos x+B \\
d y=-A \sin x d x \\
A \sin x=+-\sqrt{A^{2}-(y-B)^{2}}
\end{array}\right| \\
& =\left(\begin{array}{c}
A \sin x<0 \Leftrightarrow A \sin x=-\sqrt{A^{2}-(y-B)^{2}} \\
A \sin x>0 \Leftrightarrow A \sin x=\sqrt{A^{2}-(y-B)^{2}}
\end{array}\right) \\
& =\int_{B-A}^{Y} \frac{d y}{2 \pi \sqrt{A^{2}-(y-B)^{2}}}-\int_{Y}^{B-A} \frac{d y}{2 \pi \sqrt{A^{2}-(y-B)^{2}}} \\
& =\int_{B-A}^{Y} \frac{d y}{\pi \sqrt{A^{2}-(y-B)^{2}}} \\
& \Rightarrow \quad p_{Y}(y)=\frac{1}{\pi \sqrt{A^{2}-(y-B)^{2}}} \\
& E[Y]=\int_{B-A}^{Y} \frac{y d y}{\pi \sqrt{A^{2}-(y-B)^{2}}}=\left|\begin{array}{c}
y-B=A \cos x \\
d y=-A \sin \alpha d \alpha \\
\alpha=\arccos \left(\frac{y-B}{A}\right)
\end{array}\right| \\
& =\frac{1}{\pi} \int_{\pi}^{0} \frac{-A \sin \alpha(B+A \cos \alpha)}{A \sin \alpha} d \alpha=\frac{1}{\pi} \int_{0}^{\pi}(B+A \cos \alpha) d \alpha \\
& \left.\Rightarrow \frac{B \alpha}{\pi}\right|_{0} ^{\pi}+\left.A \sin \alpha\right|_{0} ^{\pi}=B
\end{aligned}
$$$$
E\left[(Y-B)^{2}\right]=\int_{B-A}^{Y} \frac{(y-B)^{2} d y}{\pi \sqrt{A^{2}-(y-B)^{2}}}=\left|\begin{array}{c}
y-B=A \cos x \\
d y=-A \sin \alpha d \alpha \\
\alpha=\arccos \left(\frac{y-B}{A}\right)
\end{array}\right|
$$ 


$$
\begin{gathered}
=\int_{\pi}^{0} \frac{-A \sin \alpha(A \cos \alpha)^{2}}{\pi A \sin \alpha} d \alpha=\frac{A^{2}}{\pi} \int_{0}^{\pi} \cos ^{2} \alpha d \alpha \\
\left.\Rightarrow \frac{A^{2}}{2 \pi}(\alpha+\sin 2 \alpha)\right|_{0} ^{\pi}=\frac{A^{2}}{2} \\
F\left[p_{Y}(x)\right]=E_{Y}\left[e^{-j \omega x}\right]=\int_{B-A}^{B+A} \frac{e^{-j \omega x} d y}{\pi \sqrt{A^{2}-(y-B)^{2}}}=\left|\begin{array}{c}
y-B=A \cos x \\
d y=-A \sin \alpha d \alpha \\
\alpha=\arccos \left(\frac{y-B}{A}\right)
\end{array}\right| \\
\Rightarrow \int_{\pi}^{0} \frac{-A \sin \alpha e^{-j \omega(B+A \cos \alpha)}}{\pi A \sin \alpha} d \alpha=\frac{e^{-j \omega B}}{\pi} \int_{0}^{\pi} e^{-j \omega A \cos \alpha} d \alpha
\end{gathered}
$$

Obs.: $J_{n}(z)=\frac{j^{-n}}{\pi} \int_{0}^{\pi} e^{-j z \cos \theta} \cos (n \theta) d \theta$, onde $\mathbf{J}_{n}(z)$ é a função de Bessel de primeiro tipo de ordem n.

Transformada de Fourier da Gaussiana:

$$
F\left[\frac{e^{-\frac{(y-\mu)^{2}}{2 \sigma^{2}}}}{\sqrt{2 \pi} \sigma}\right]=e^{-j \omega \mu-\frac{1}{2} \omega^{2} \sigma^{2}}
$$

Representação alternativa para a função $\delta$ de Dirac:

$$
\delta(x)=\frac{1}{2 \pi} \int_{-\infty}^{\infty} e^{-i k x} d k .
$$




\section{Capítulo 4}

\section{Resultados Experimentais}

\subsection{Introdução}

Neste capítulo é descrita a montagem experimental realizada a fim de testar a validade da teoria desenvolvida neste trabalho e os resultados obtidos são apresentados e comentados.

A idéia da experiência é simular um sistema com ruído interferométrico e fazer o levantamento das estatísticas de taxa de bits errados a fim de que sejam comparadas com as taxas previstas teoricamente.

\subsection{Descrição da montagem}

Equipamentos e componentes utilizados:

i) Medidor de taxa de bits errados ( BER meter ) Anritsu ME 3620A, transmissor (TX) e receptor $(\mathrm{RX})$.

ii) Medidor de Potência Ótica Anritsu ML 910B (PM).

iii) Atenuador Programável Ótico Anritsu MN 9610B (A1). 
iv) Um atenuador variável Eigenlight Power Monitor 420 (A2).

v) Analisador de Espectro HP 8594E.

vi) Um foto-receptor de baixo ruído de $1 \mathrm{GHz}$.

vii) Dois isoladores óticos.

viii) Uma chave ótica.

ix) Vários acopladores óticos 2x2.

x) Dois acopladores óticos 1x16.

xi) 16 controladores de polarização.

O esquema da montagem pode ser visto na figura 4.1. Cada um dos 16 braços que simulam um multi-percurso possuem comprimentos que variam desde aproximadamente $3 m$ para o primeiro braço, até $3+16 \times 3=51 m$ para o décimo sexto. $\mathrm{O}$ valor aproximado de $3 m$ foi escolhido de modo a garantir que o bit que irá interferir no caminho alternativo seja diferente do bit passando no caminho direto.

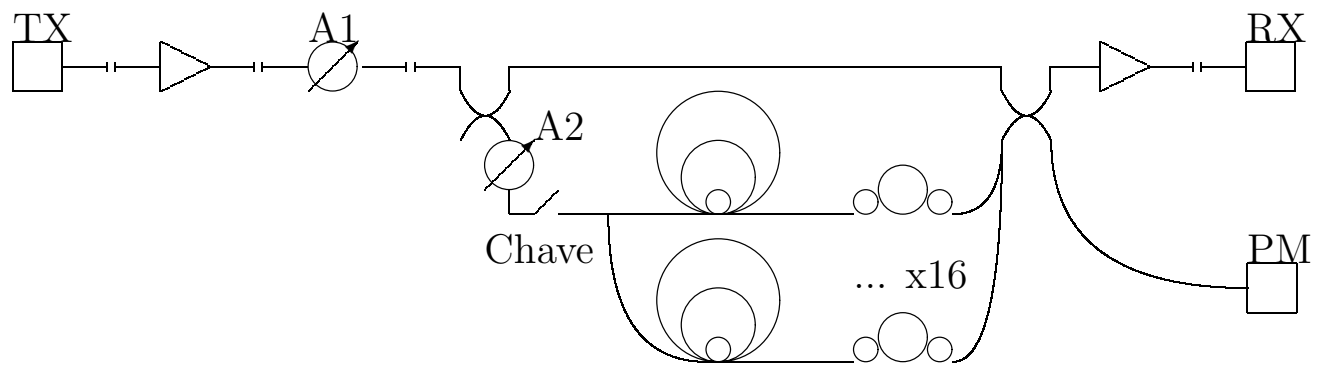

Figura 4.1: Esquema do experimento 


\subsection{Levantamento do parâmetro $\alpha$}

O parâmetro importante a ser medido e variado neste experimento é o valor da atenuação dos sinais de multi-percurso ( crosstalks ) em relação ao sinal do braço principal - o parâmetro $\alpha$. É fundamental que esse parâmetro seja medido corretamente, pois o resultado da medida é muito sensível a variações em $\alpha$. O procedimento adotado para o caso de um crosstalk foi o seguinte: em primeiro lugar, eliminou-se o sinal do braço de crosstalk e mede-se a potência ótica de saída. Em segundo lugar, eliminou-se o sinal do braço de principal e mediu-se novamente a potência ótica na saída. A relação entre essas potências é o valor do parâmetro $\alpha$. É importante frisar que esta medida tem que ser realizada no braço do interferômetro que está ligado ao medidor de taxa de erros, pois esta relação de potências é diferente nos dois braços de saída, podendo acarretar erros da ordem de $1 \mathrm{~dB}$.

No caso de dois ou mais crosstalks, o procedimento adotado foi semelhante, mas existe o problema de que o parâmetro $\alpha$ é ligeiramente diferente para cada braço de crosstalk. Desta forma, foi adotado um parâmetro "médio", medindo-se a potência ótica de saída com apenas o braço principal eliminado, deixando todos os caminhos de crosstalk. O valor de $\alpha$ é obtido a partir da relação de potência entre o caminho principal e os caminhos de crosstalk, subtraido de $10 \log N$, onde $N$ é o número de crosstalks. Isto supõe uma potência média igual para todos os caminhos. 


\subsection{Número médio de erros, precisão da medida e tempo necessário}

Seja $\left\{x_{i}\right\}$ uma coleção de $N$ variáveis aleatórias independentes definidas da seguinte forma:

$$
\begin{cases}x_{i}=0 & \Leftrightarrow \text { não houve erro no i-ésimo bit transmitido } \\ x_{i}=1 & \Leftrightarrow \text { houve erro no i-ésimo bit transmitido }\end{cases}
$$

Assim, $x_{i}$ é uma variável aleatória de Bernoulli, onde $P\left[x_{i}=1\right]=p$. O valor esperado de $x_{i}$ vale,

$$
E\left[x_{i}\right]=0 \cdot(1-p)+1 \cdot p=p .
$$

Seja então

$$
N_{e}=\sum_{i=1}^{N} x_{i}
$$

$N_{e}$ é o número de bits errados e da forma como foi definido é uma variável aleatória binomial com parâmetros $\mathrm{N}$ e p. A $B E R$ pode ser escrita como

$$
B E R=\frac{N_{e}}{N}=\frac{1}{N} \sum_{i=1}^{N} x_{i}
$$

Portanto, a $B E R$ é uma variável aleatória tal que

$$
\begin{aligned}
E[B E R] & =E\left[\frac{1}{N} \sum_{i=1}^{N} x_{i}\right] \\
\text { (Linearidade de } E[\cdot]) & =\frac{1}{N} \sum_{i=1}^{N} E\left[x_{i}\right] \\
\left(E\left[x_{i}\right]=p\right) & =\frac{1}{N} \sum_{i=1}^{N} p \\
& =\frac{1}{N} \cdot N \cdot p
\end{aligned}
$$




$$
=p
$$

e

$$
\begin{aligned}
V[B E R] & =V\left[\frac{1}{N} \sum_{i=1}^{N} x_{i}\right] \\
& =\frac{1}{N^{2}} \cdot V\left[\sum_{i=1}^{N} x_{i}\right] \\
\text { (independência entre os } \left.x_{i}{ }^{\prime} \mathrm{s}\right) & =\frac{1}{N^{2}} \sum_{i=1}^{N} V\left[x_{i}\right] \\
& =\frac{1}{N^{2}} \sum_{i=1}^{N} p(1-p) \\
& =\frac{1}{N^{2}} \cdot N \cdot p(1-p) \\
& =\frac{p(1-p)}{N} .
\end{aligned}
$$

A expressão obtida para a variância da $B E R$ mostra que seu desvio padrão é proporcional ao inverso da raiz quadrada do número de bits transmitidos. Suponha então que seja adotada a seguinte meta: que o desvio padrão da medida da $B E R$ seja menor que uma certa fração $t$ do valor esperado da $B E R$, isto é:

$$
\begin{aligned}
\sigma_{B E R}<t \cdot E[B E R] & \Leftrightarrow \sqrt{p \frac{(1-p)}{N}}<t \cdot p \\
& \Leftrightarrow \sqrt{\frac{(1-p)}{p N}}<t \\
& \Leftrightarrow \frac{(1-p)}{p N}<t^{2} \\
(p \ll 1) & \Leftrightarrow N>\frac{(1-p)}{p t^{2}} \approx \frac{1}{p t^{2}}
\end{aligned}
$$

Chame $t$ de "precisão da medida". A expressão 4.4.7 permite relacionar a precisão com o número de bits transmitidos, porém permanece uma dependência do valor 
esperado da BER $(p)$. Essa expressão pode ser reescrita em função do valor esperado do número de bits errados:

$$
\begin{aligned}
N>\frac{1}{E[B E R] t^{2}} & \Leftrightarrow N E[B E R]>\frac{1}{t^{2}} \\
& \Leftrightarrow E\left[N_{e}\right]>\frac{1}{t^{2}}
\end{aligned}
$$

Na tabela 4.1 é possível observar a variação da precisão em função do valor esperado do número de bits errados.

Tabela 4.1: Variação da Precisão em função do valor esperado do número de bits errados

\begin{tabular}{|c|c|}
\hline Número médio de erros & Precisão(\%) \\
\hline 10 & 31.62 \\
\hline 20 & 22.36 \\
\hline 50 & 14.14 \\
\hline 100 & 10.00 \\
\hline 200 & 7.07 \\
\hline 500 & 4.47 \\
\hline 1000 & 3.16 \\
\hline
\end{tabular}

Decorre daí a pergunta: quanto tempo leva para obter-se uma medida com uma determinada precisão? Seja $B$ a taxa de bits transmitidos e seja $T$ o tempo decorrido.

$$
\begin{aligned}
N>\frac{1}{p t^{2}} & \Leftrightarrow B T>\frac{1}{p t^{2}} \\
& \Leftrightarrow T>\frac{1}{B p t^{2}}
\end{aligned}
$$

Usando como exemplo a precisão de $10 \%$, isto é, um valor esperado de 100 para o número de erros e $\mathrm{B}=2.5$ Gigabits por segundo, tem-se:

$$
T=\frac{N}{B}=\frac{E\left[N_{e}\right]}{B \cdot E[B E R]}=\frac{100}{2.5 \times 10^{9} \cdot p}=\frac{4.0 \times 10^{-8}}{p}
$$


É possível então construir uma tabela com a duração de cada medida em função do número médio de erros necessários para se chegar a uma determinada precisão, conforme a tabela 4.2 .

Tabela 4.2: Tempo de medida para 10\% (100 erros)

\begin{tabular}{|c|c|}
\hline \multicolumn{2}{|c|}{ Tempo de medida para 10\% (100 erros) } \\
\hline $\mathbf{E}[\mathbf{B E R}]$ & $\mathbf{T}$ \\
\hline $1.0 \times 10^{-2}$ & $4.0 \mathrm{us}$ \\
\hline $1.0 \times 10^{-3}$ & $40.0 \mathrm{us}$ \\
\hline $1.0 \times 10^{-4}$ & $400.0 \mathrm{us}$ \\
\hline $1.0 \times 10^{-5}$ & $4.0 \mathrm{~ms}$ \\
\hline $1.0 \times 10^{-6}$ & $40.0 \mathrm{~ms}$ \\
\hline $1.0 \times 10^{-7}$ & $400.0 \mathrm{~ms}$ \\
\hline $1.0 \times 10^{-8}$ & $4.0 \mathrm{~s}$ \\
\hline $1.0 \times 10^{-9}$ & $40.0 \mathrm{~s}$ \\
\hline $1.0 \times 10^{-10}$ & $400.0 \mathrm{~s}=0 \mathrm{~h}, 6$ min e $40 \mathrm{~s}$ \\
\hline $1.0 \times 10^{-11}$ & $4.0 \mathrm{ks}=1 \mathrm{~h}, 6$ min e $40 \mathrm{~s}$ \\
\hline $1.0 \times 10^{-12}$ & $40.0 \mathrm{ks}=11 \mathrm{~h}, 6 \mathrm{~min}$ e $40 \mathrm{~s}$ \\
\hline
\end{tabular}

\subsection{Estratégia da medida}

A idéia aqui é levantar a curva BER x RSR do sistema em questão. A experiência em sí nos fornece uma curva BER x Potência. A fim de aproveitar a mesma montagem, duas curvas são levantadas de cada vez. Uma é a curva com ruído interferométrico, e a outra é uma curva back-to-back, isto é, uma ligação direta, sem o ruído interferométrico. Nesta última situação, apenas o ruído térmico influencia na taxa de bits errados.

O maior problema com a aparelhagem disponível é que a medida da taxa de bits errados depende significativamente da temperatura ambiente. Não existe uma 
compensação interna no aparelho de recepção para o ruído térmico do fotodetetor. Para uma mesma potência recebida, diferentes taxas de bits errados serão obtidas, em diferentes horas do dia. Desta forma é necessário realizar uma compensação deste ruído térmico, para que a medida tenha algum significado.

A compensação é feita medindo-se no final de cada medida a taxa de bits errados para uma determinada potência fixa. Supondo que a variação desta taxa ocorre exclusivamente devido à variação do ruído térmico no receptor, e supondo esse ruído gaussiano, basta utilizar a equação 2.4.10 com $N=0$ para obter

$$
\begin{aligned}
B E R_{i}^{r e f}=Q\left(\frac{\zeta_{i}^{r e f}}{2}\right) & \Rightarrow \zeta_{i}^{r e f}=2 \cdot Q^{-1}\left(B E R_{i}^{r e f}\right) \\
& \Rightarrow \frac{P_{i}^{r e f}}{\sigma_{T i}}=2 \cdot Q^{-1}\left(B E R_{i}^{r e f}\right) \\
& \Rightarrow \sigma_{T i}^{r e f}=\frac{P_{i}^{r e f}}{2 \cdot Q^{-1}\left(B E R_{i}^{r e f}\right)}
\end{aligned}
$$

onde $P_{i}^{r e f}$ é a potência de referência medida no braço do medidor de potência.

Com este valor de $\sigma_{T}$, é possível calcular a relação sinal-ruído ótica da i-ésima medida pela expressão:

$$
\zeta_{i}=\frac{P_{i}}{\sigma_{T i}}=\frac{P_{i}}{P_{i}^{r e f}} \cdot 2 \cdot Q^{-1}\left(B E R_{i}^{r e f}\right)
$$

Note ainda que o procedimento descrito acima é imune a um desvio fixo em dB de potência no medidor de potência ( offset ). Tal desvio existe na prática, pois a potência medida no braço do medidor de potência é ligeiramente diferente da potência medida no braço do medidor de taxa de erros. 


\subsection{O problema da responsividade do sensor}

Observe o gráfico da figura 4.2. As duas curvas mais à esquerda são as curvas de probabilidade de erro back-to-back do sistema teórica ( curva maior) e medida ( curva menor ). As quatro curvas à direita são as curvas de probabilidade de erro do sistema com ruído interferométrico com parâmetros $\alpha$ de -13, -14 e -15 dB, e a curva medida para -14 dB. Note que a curva back-to-back medida cruza a teórica assim como a curva medida para -14 dB. Este comportamento é completamente incompatível com a teoria, pois na situação back-to-back apenas o ruído térmico estaria atuando, e uma curva como mostrada na figura cruza a curva teórica do sistema funcionando apenas com o ruído branco gaussiano. 


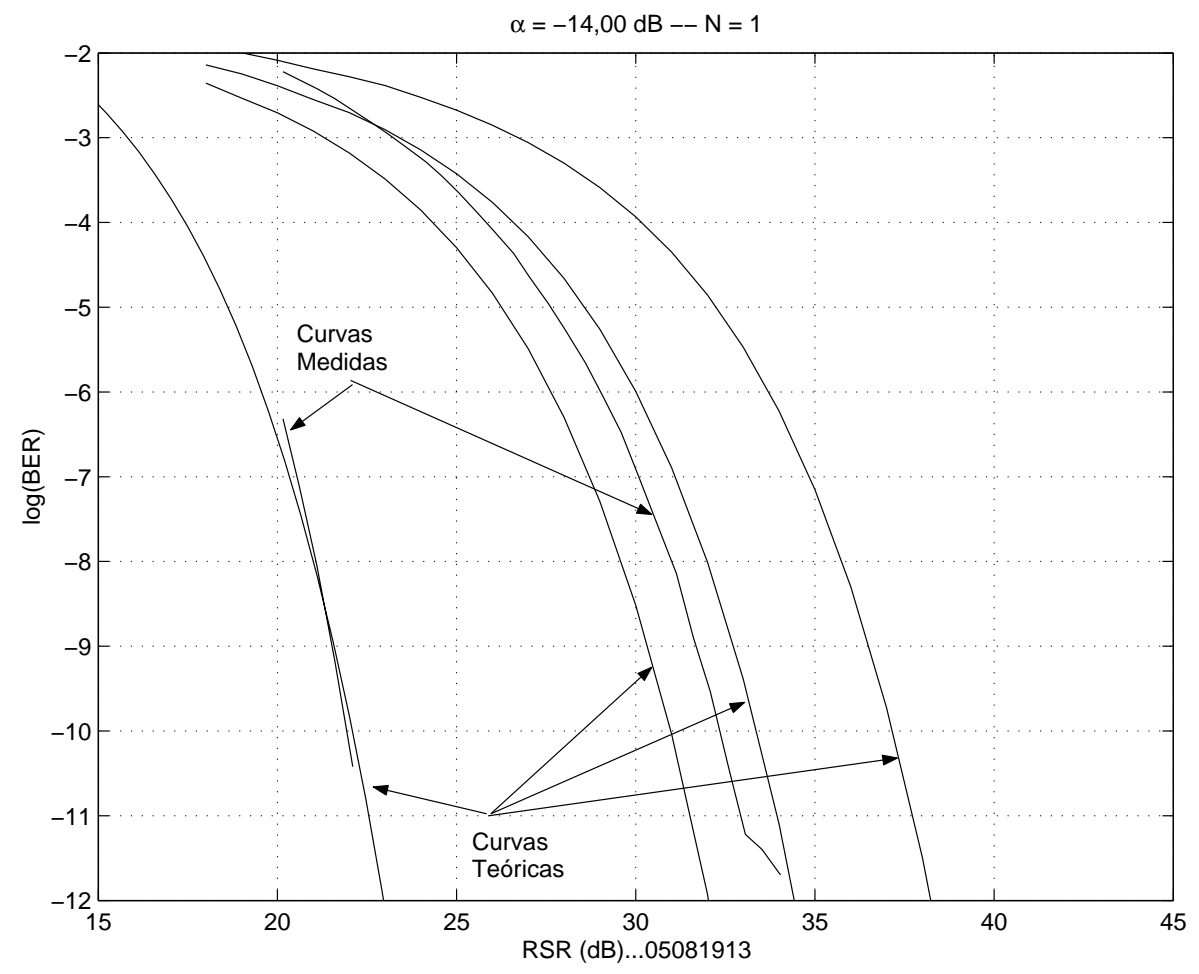

Figura 4.2: Gráfico da probabilidade de erro em função da relação sinal-ruído elétrica, sem compensação de responsividade

Este comportamento pode ser explicado através da hipótese de que a resposta de corrente do sensor não é linear com a potência recebida. No artigo [9] de Christian Hentschel, pode-se observar documentado esse efeito de "supra-linearidade". Para o autor, este efeito não foi ainda estudado cuidadosamente, e é gerado por um aumento da responsividade do sensor, tipicamente para níveis de potência da ordem de 100 $\mu W$. 
Seja então $R$ a responsividade do sensor. Suponha que $R$ é uma função de $P$. Assim, pode-se escrever que para a i-ésima medida:

$$
\begin{aligned}
P_{i}^{e f}=R\left(P_{i}\right) \cdot P_{i} & \Rightarrow \zeta_{i}=\frac{P_{i}^{e f}}{\sigma_{T i}} \\
& \Rightarrow \zeta_{i}=\frac{R\left(P_{i}\right) \cdot P_{i}}{\sigma_{T i}}
\end{aligned}
$$

Uma hipótese simples para a função $R(P)$ é que ela seja uma correção linear no logaritmo, ou seja:

$$
\begin{aligned}
R(P)(d B)=k \cdot\left(P(d B)-P_{0}(d B)\right) & \Rightarrow 10^{\frac{R(P)(d B)}{10}}=10^{\frac{k \cdot\left(P(d B)-P_{0}(d B)\right)}{10}} \\
& \Rightarrow R=\left(\frac{P}{P_{0}}\right)^{k}
\end{aligned}
$$

Note que $R\left(P_{0}\right)=1$, portanto se tomarmos $P_{0}=P_{\text {ref }}$, onde $P_{\text {ref }}$ é a potência de referência utilizada para medir o valor da variância do ruído térmico, a expressão da variância do ruído térmico no receptor desenvolvida na seção anterior permanece válida. A expressão da relação sinal-ruído ótica, porém, passa a ser

$$
\zeta_{i}^{\prime}=R \cdot \zeta_{i}=\frac{R\left(P_{i}\right) \cdot P_{i}}{\sigma_{T i}}
$$

Através de várias observações, foi possível chegar a um valor de $k=0.2$. Na figura 4.3 estão mostrados os mesmos dados mostrados na figura 4.2, mas com a correção da relação sinal ruído dada pela equação 4.6.1. Note como as curvas das medidas tanto para o sistema back-to-back quanto para o sistema com ruído interferométrico ficaram paralelas às curvas teóricas. 


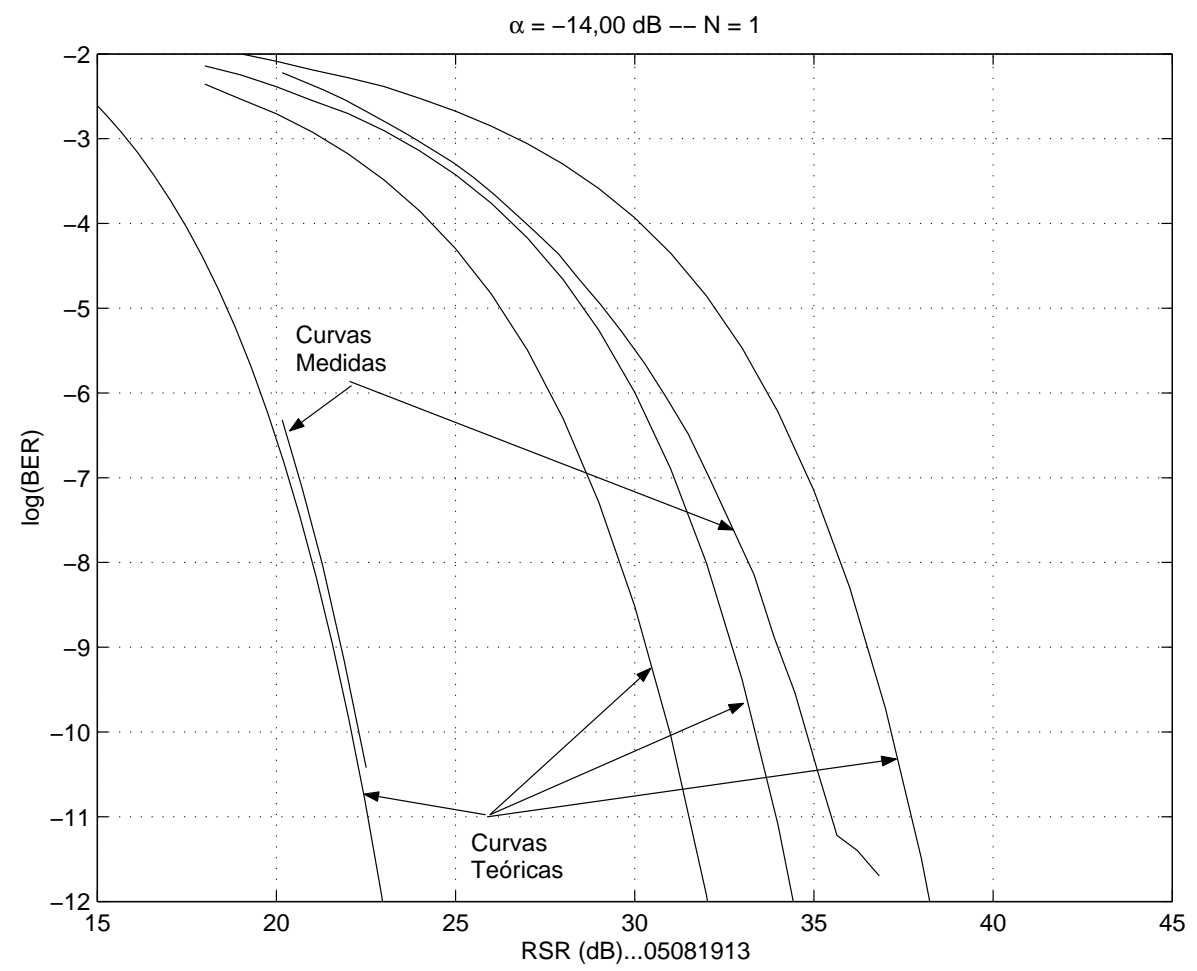

Figura 4.3: Gráfico da probabilidade de erro em função da relação sinal-ruído elétrica, com compensação de responsividade

Note que apesar de todas as correções feitas nas medidas, a curva back-to-back ainda apresenta um pequeno deslocamento entre o valor teórico e o medido. Uma possibilidade para explicar essa penalidade seria através da razão de extinção do laser do aparelho transmissor. A razão de extinção é a razão entre a potência emitida pelo laser quando em nível alto e a potência emitida pelo laser quando em nível baixo. Isto ocorre porque devido às altas taxas de transmissão envolvidas, o laser nunca consegue desligar ou ligar completamente. 
A expressão da penalidade devido à razão de extinção é dada por [1]

$$
P=10 \log \frac{1+r}{1-r}
$$

onde $r$ é a razão de extinção. A medida deste parâmetro já havia sido realizada anteriormente no laboratório e o valor obtido foi de $22 \mathrm{~dB}$. Isto implica numa penalidade de 0,055 dB. Uma ampliação no gráfico da figura 4.3 mostra que a penalidade é da ordem de $0.3 \mathrm{~dB}$, o que significa que a razão de extinção não é explicação suficiente. Infelizmente a documentação do medidor de taxa de erros é pobre em relação às suas limitações técnicas, e certamente existem outras penalidades envolvidas nesta medida.

\subsection{Resultados obtidos para um crosstalk}

Foram realizadas medidas para diversos valores do parâmetro $\alpha$. A fim de aumentar a faixa dinâmica de medidas no arranjo experimental com um caminho de crosstalk, foram utilizados dois divisores 2 × 2 ao invés de dois divisores 1 x 16 . Deste modo foi possível medir valores de $-13 \mathrm{~dB}$ até $33 \mathrm{~dB}$, variando de $1 \mathrm{em} 1 \mathrm{~dB}$. Na figura 4.4 pode-se ver os histogramas obtidos para os níveis 0 e 1 do sinal com ruído interferométrico. Note que a distribuição de dois picos ocorre apenas quando um nível alto interfere com um nível alto. 


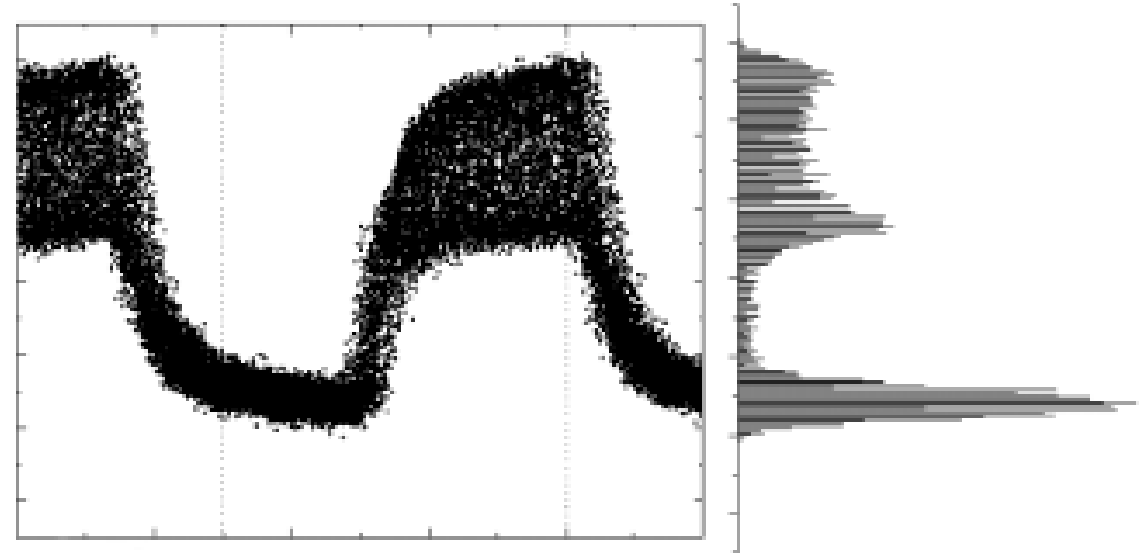

Figura 4.4: Histograma do sinal

Nas figuras a seguir, tentou-se colocar as curvas medidas em comparação com a previsão teórica. Nota-se um erro da ordem de $1 \mathrm{~dB}$, o que foi considerado bastante bom. 


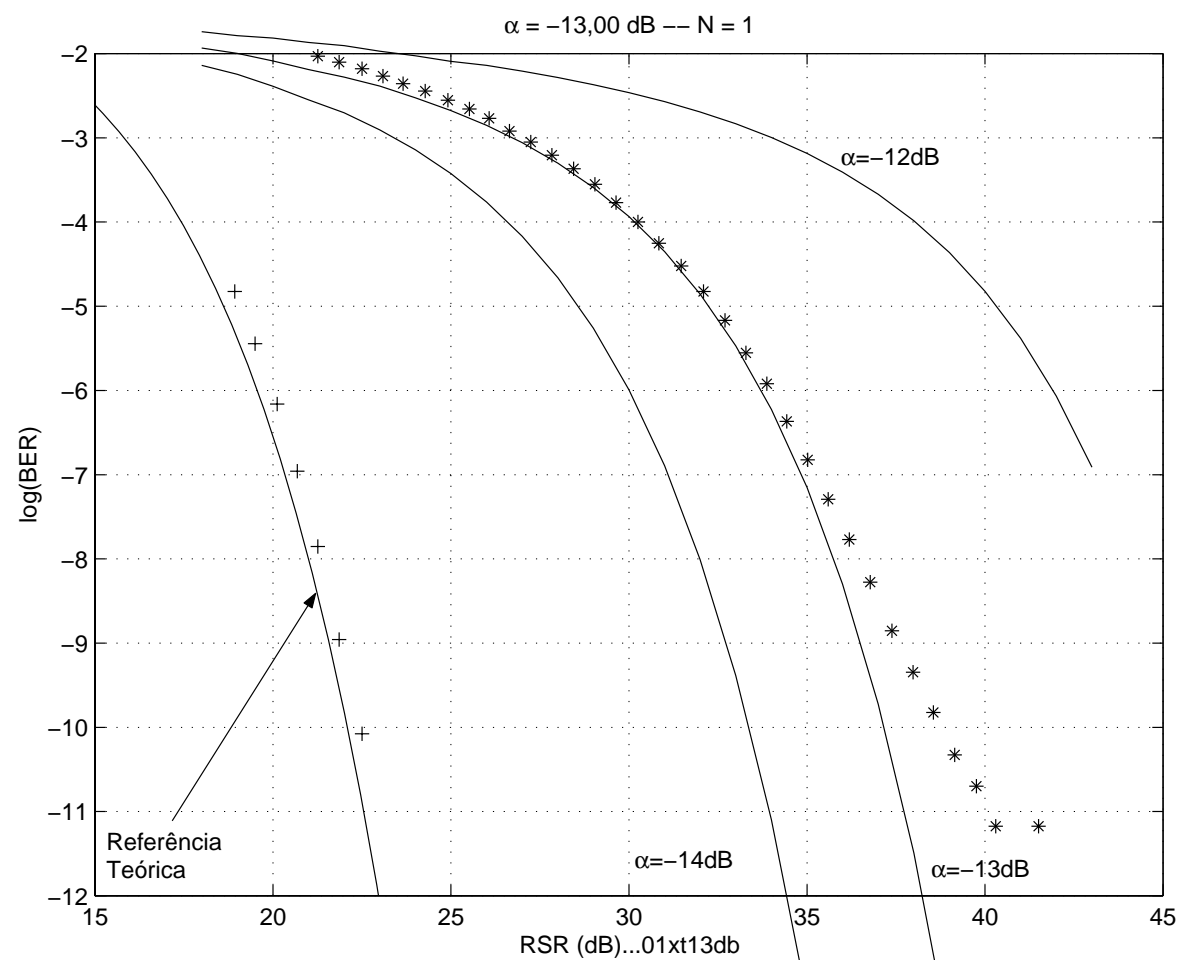

Figura 4.5: Comparação dos valores teóricos e medidos da probabilidade de erro em função da relação sinal-ruído elétrica, 1 crosstalk, $\alpha=-13 \mathrm{~dB}$ 


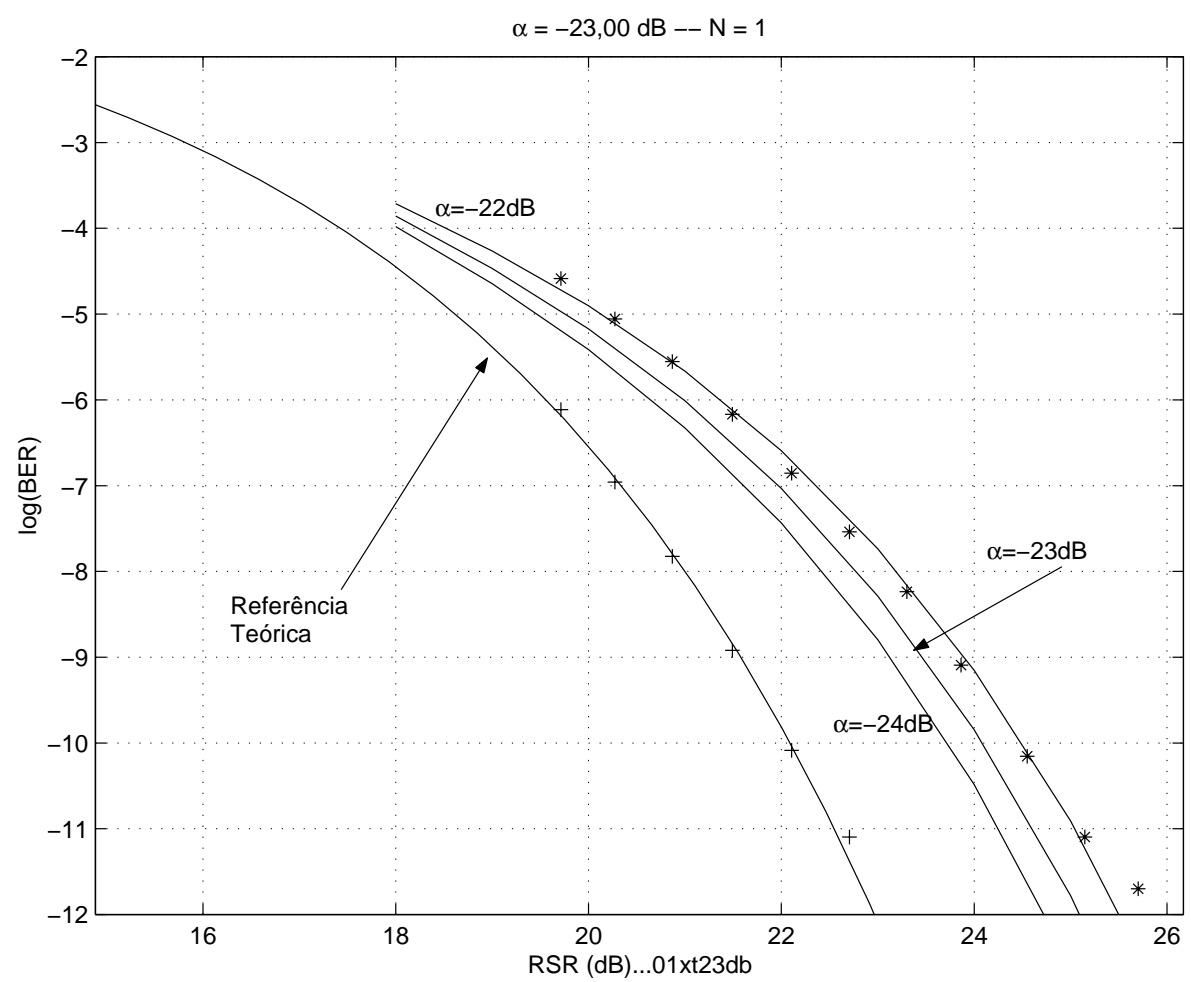

Figura 4.6: Comparação dos valores teóricos e medidos da probabilidade de erro em função da relação sinal-ruído elétrica, 1 crosstalk, $\alpha=-23 \mathrm{~dB}$ 


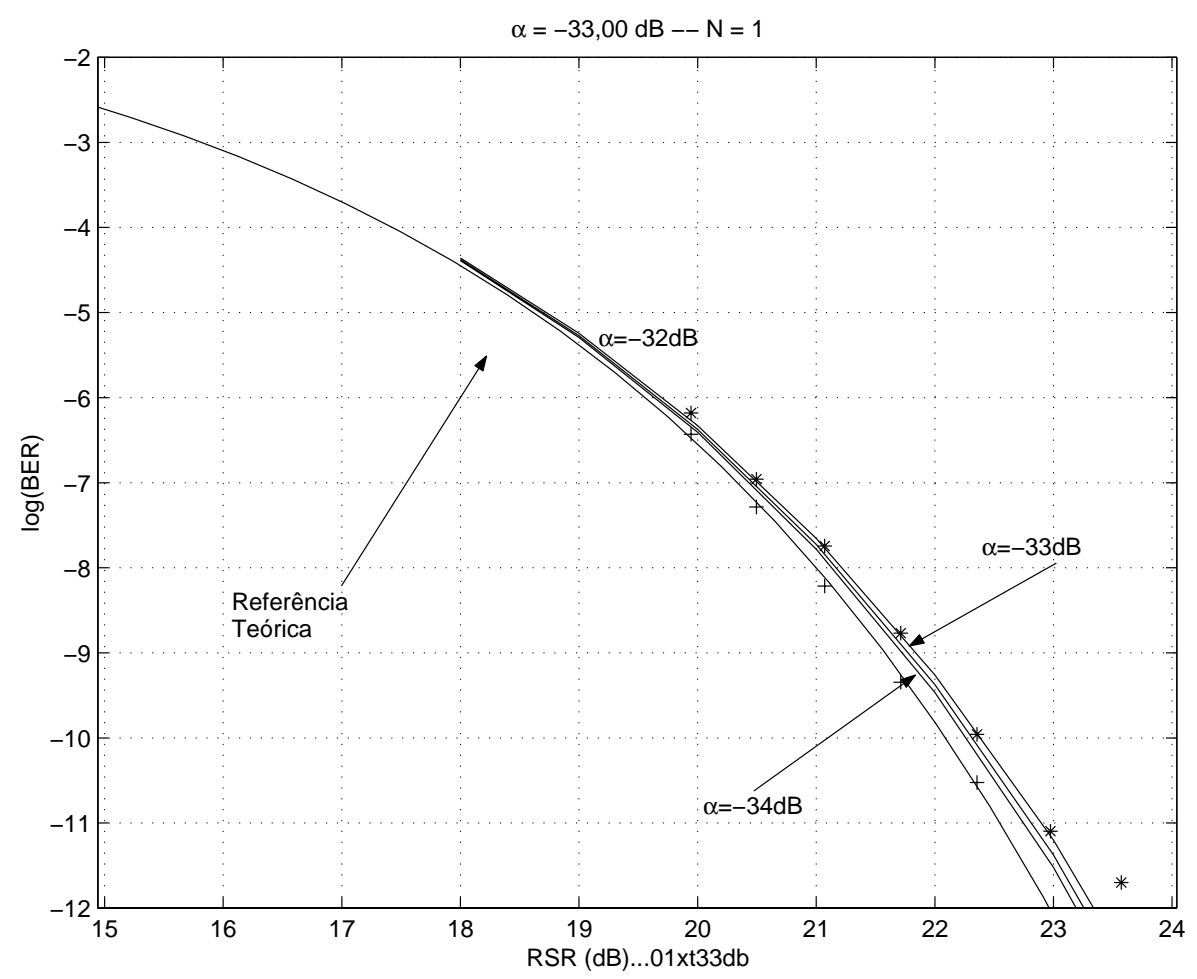

Figura 4.7: Comparação dos valores teóricos e medidos da probabilidade de erro em função da relação sinal-ruído elétrica, 1 crosstalk, $\alpha=-33 \mathrm{~dB}$

\subsection{Resultados obtidos para dois crosstalks}

No caso de dois crosstalks, devido à presença de mais dois divisores no caminho ótico, totalizando 4 divisores $2 \times 2$, o menor valor de $\alpha$ medido foi - $18.32 \mathrm{~dB}$. O mesmo procedimento da medida anterior foi adotado, sendo levantadas as curvas de 1 em 1 dB. As figuras a seguir são análogas em conteúdo às da seção anterior. A dificuldade 
encontrada nessa medida foi a regulagem dos controladores de polarização dos multipercursos, que foi feita de forma a maximizar simultâneamente a taxa de bits errados para uma dada potência. Nota-se aqui um erro da ordem de 2 dB.

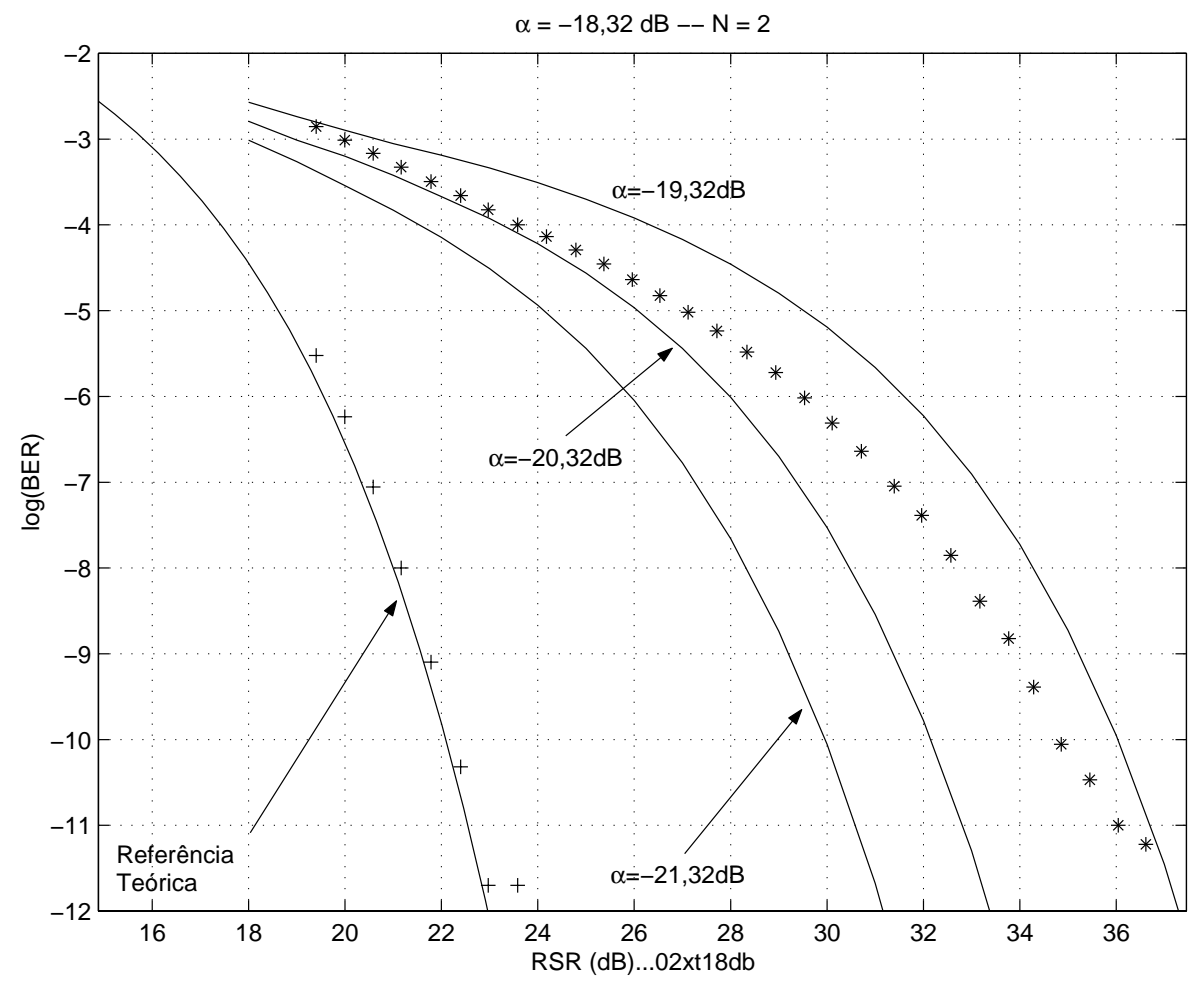

Figura 4.8: Comparação dos valores teóricos e medidos da probabilidade de erro em função da relação sinal-ruído elétrica, 2 crosstalks, $\alpha=-18.32 \mathrm{~dB}$ 


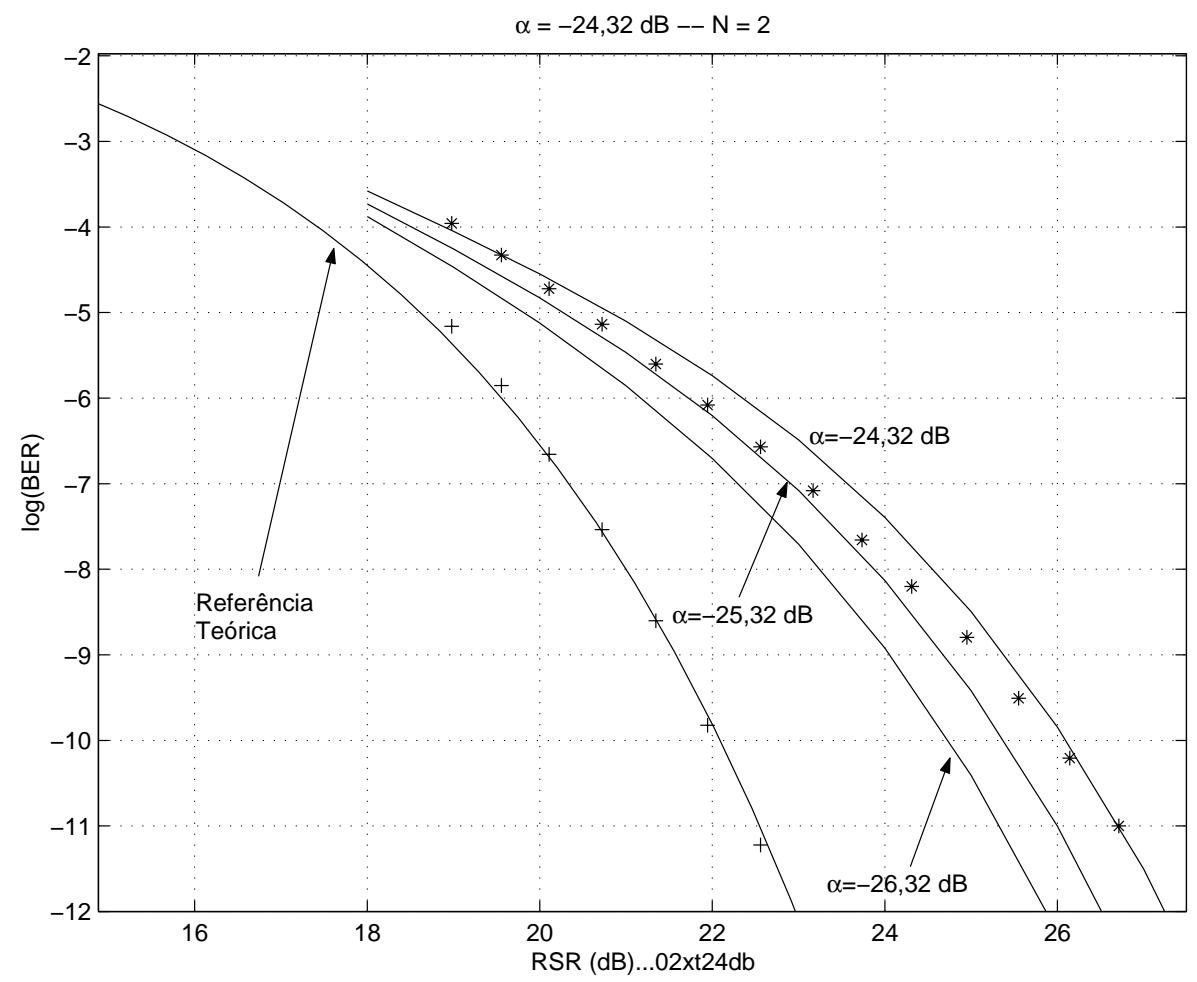

Figura 4.9: Comparação dos valores teóricos e medidos da probabilidade de erro em função da relação sinal-ruído elétrica, 2 crosstalks, $\alpha=-24.32 \mathrm{~dB}$ 


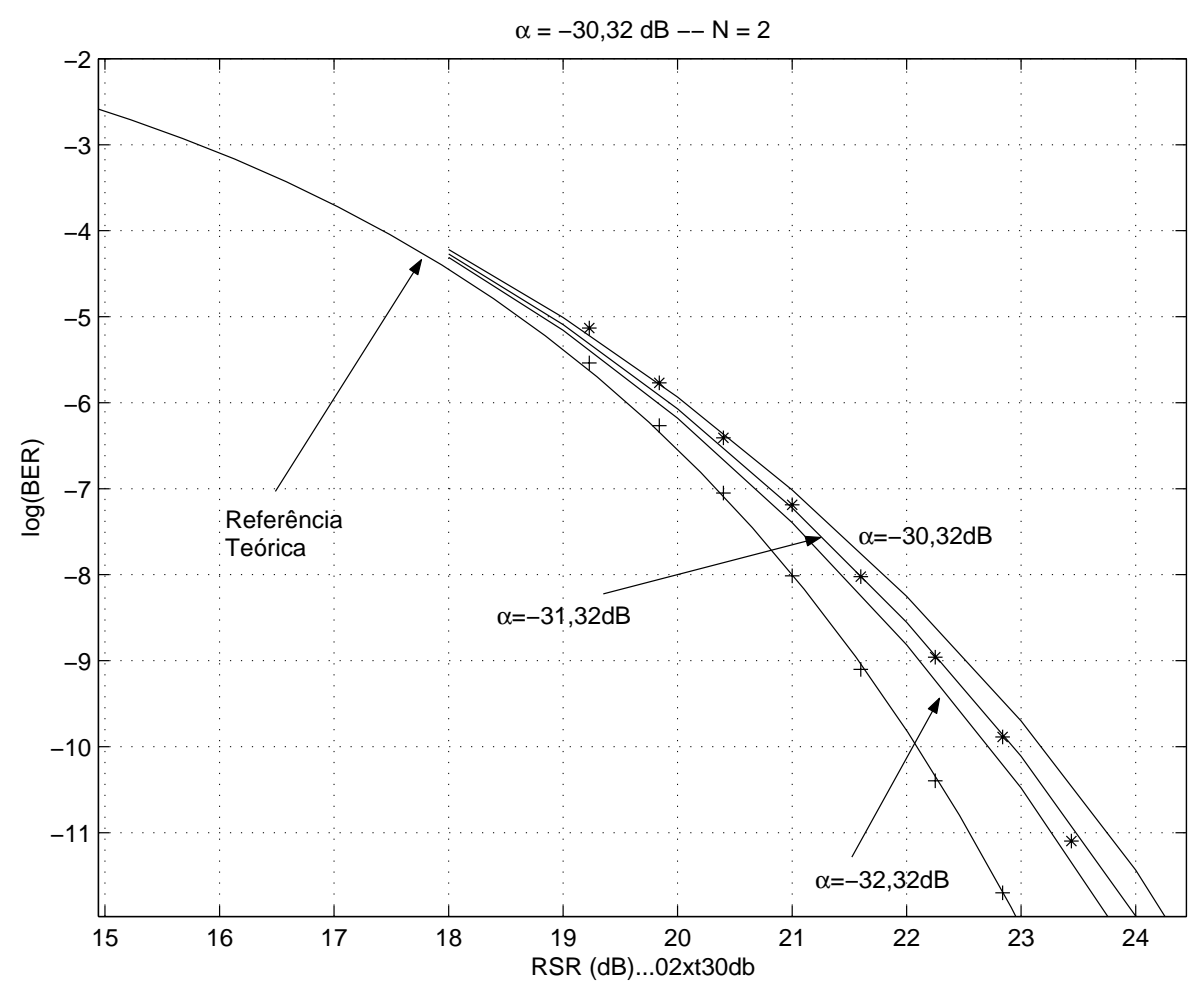

Figura 4.10: Comparação dos valores teóricos e medidos da probabilidade de erro em função da relação sinal-ruído elétrica, 2 crosstalks, $\alpha=-30.32 \mathrm{~dB}$

\subsection{Resultados obtidos para 16 crosstalks}

Neste caso, como não se possuíam 16 controladores de polarização, optou-se por maximizar a interferência através de um controlador de polarização no braço do caminho principal. Nota-se um erro da ordem de $3 \mathrm{~dB}$, que pode ser considerado excelente, uma vez que as polarizações não foram individualmente acertadas. Apenas o caso de 
$\alpha=31.45 \mathrm{~dB}$ foi medido.

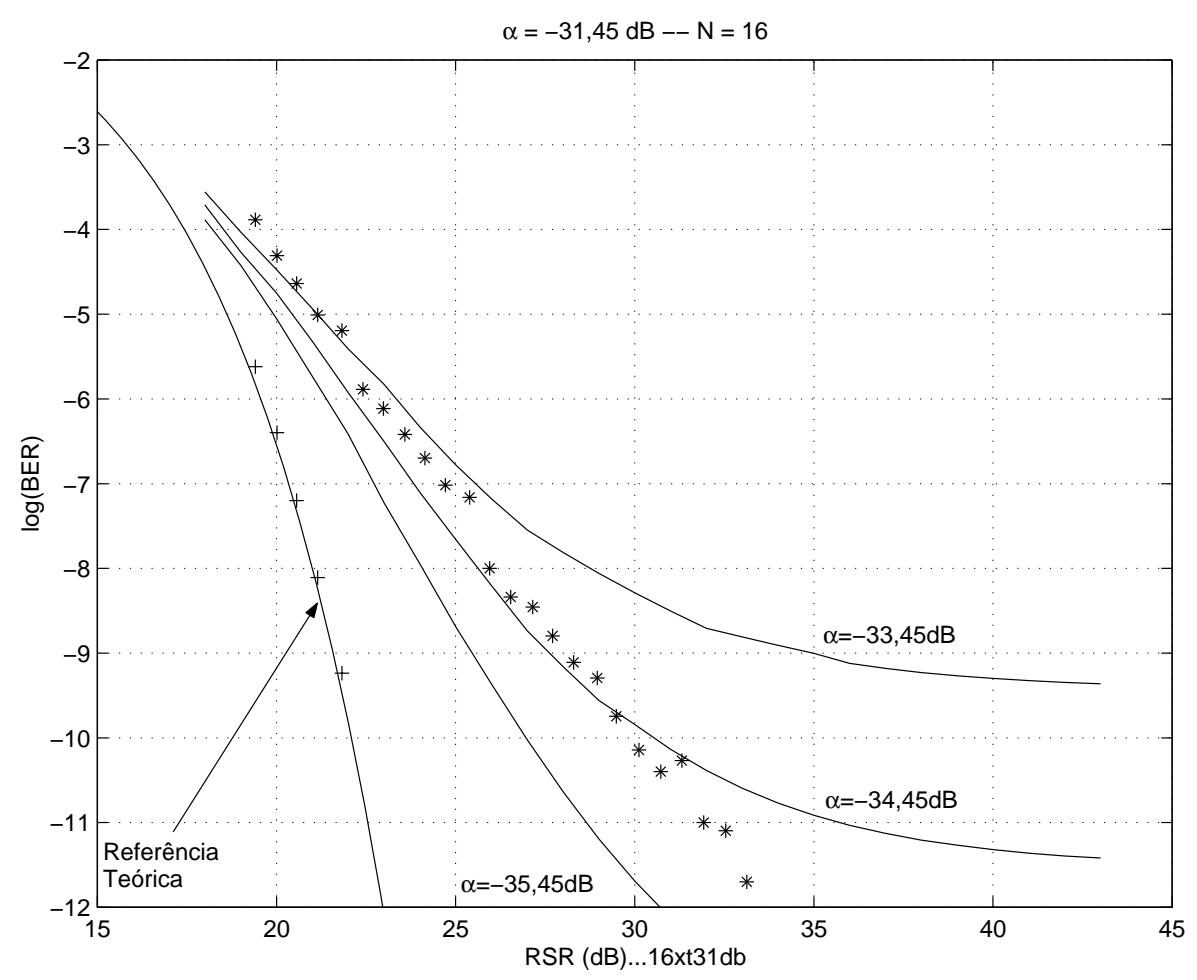

Figura 4.11: Comparação dos valores teóricos e medidos da probabilidade de erro em função da relação sinal-ruído elétrica, 16 crosstalks, $\alpha=-31.45 \mathrm{~dB}$

\subsection{Conclusões}

Podem se considerar muito bons os resultados experimentais obtidos. As medidas obtidas para o caso de um crosstalk apresentaram um erro de menos de $1 \mathrm{~dB}$ em relação às curvas teóricas, enquanto as medidas para dois crosstalks apresentaram 
um erro menor que $2 \mathrm{~dB}$. A medida para o caso de 16 crosstalks é mais difícil de ser realizada, pois exigiria a presença de 16 controladores de polarização. Mesmo sem um ajuste fino da polarização, foi obtido um erro de $3 \mathrm{~dB}$ em média.

As características internas do medidor de taxa de erros não estavam documentadas no manual do equipamento, em particular as características de responsividade do sensor tiveram que ser avaliadas na prática da melhor forma possível.

Infelizmente não foi possível medir experimentalmente os valores de chão das probabilidades de bits errados, a fim de comparar os resultados com a teoria do capítulo 2. Tal medida iria requerer uma potência de laser que não estava disponível. Outro fator a ser considerado é que as expressões do capítulo 2 assumem uma distribuição gaussiana, o que só deve ser válido a partir de um número grande de crosstalks ( 8 por exemplo ). A opção de introduzir um amplificador, além de complicar a montagem experimental, poderia introduzir dependências de outros parâmetros no resultado final. 


\section{Capítulo 5}

\section{Conclusão}

Uma nova teoria matemática, a das variáveis aleatórias cíclicas, foi desenvolvida a fim de atribuir correta e coerentemente probabilidades às variáveis angulares. Esta teoria foi utilizada no caso particular do estudo do ruído interferométrico em sistemas óticos, porém certamente existem outras aplicações, como por exemplo na análise de crosstalk em sistemas WDM.

A teoria das variáveis aleatórias cíclicas foi desenvolvida neste trabalho apenas o suficiente para prover os resultados necessários. A característica compacta da imagem deste tipo de variável aleatória lhe confere uma estrutura bastante diferente dos modelos de variáveis aleatórias reais convencionais, de modo que outros resultados podem ser retirados desta estrutura matemática, e é certamente um caminho de continuação deste trabalho.

O modelo gaussiano de ruído interferométrico foi cuidadosamente revisado e estendido, e os resultados obtidos foram aplicados no cálculo dos valores de chão para a taxa de erros de bits em sistemas óticos. Infelizmente o aparato experimental disponível não permitiu averiguar experimentalmente as previsões desta teoria. O laser 
do transmissor do medidor de taxa de bits errados não tinha potência suficiente. Seria necessário utilizar um amplificador ótico para se chegar aos valores de relação sinal ruído onde ocorrem os valores de chão. Este também é um caminho para continuar este trabalho.

O modelo não-gaussiano foi desenvolvido utilizando a teoria matemática das variáveis aleatórias cíclicas, e assim foram obtidos resultados analíticos que na teoria de probabilidade tradicional seriam mais difíceis de se obter.

De posse das expressões analíticas para os modelos gaussiano e não-gaussiano, um programa de computador foi desenvolvido a fim de realizar o cálculo das densidades de probabilidade associadas a cada um dos modelos, para que fosse possível obter e comparar as curvas de penalidade de potência do sistema analisado. Uma outra possível continuação deste trabalho seria criar uma interface deste programa com o usuário, a fim de facilitar o seu uso.

A simulação por computador mostrou claramente que quando o número de crosstalks cresce, as curvas do modelo não-gaussiano aproximam-se das curvas do modelo gaussiano, de onde se pode concluir que o modelo gaussiano tem utilidade nos casos em que o número de crosstalks é elevado, como é o caso das redes óticas transparentes. Infelizmente não estavam disponíveis controladores de polarização suficientes para se testar na prática essa aplicação do modelo gaussiano, e aqui mais uma vez existe espaço para a continuação.

Os resultados experimentais mostraram uma concordância considerada muito boa com a teoria - menos de $1 \mathrm{~dB}$ para um crosstalk, da ordem de $2 \mathrm{~dB}$ para dois crosstalks. A grande dificuldade experimental foi que o aumento do número de crosstalks implica numa perda de dinâmica do sistema. O uso de um amplificador ótico externo poderia 
alterar de algum modo o resultado do experimento [24], de modo que essa opção não foi utilizada. Outra dificuldade era, como já foi citado anteriormente, o elevado número de controladores óticos necessários para a realização do experimento com 16 crosstalks. Como este recurso não estava disponível, a experiência foi realizada de forma um pouco mais precária, apenas para se ter uma noção do resultado em comparação com a teoria. Mesmo assim houve um erro da ordem de $3 \mathrm{~dB}$ na previsão neste caso, o que pode ser considerado bom dadas as circunstâncias.

Um grande conhecimento a respeito do equipamento de medidas também foi adquirido em conseqüência deste arranjo experimental. Infelizmente as especificações técnicas destes aparelhos não são suficientes para medidas mais sofisticadas em laboratório. 


\section{Referências Bibliográficas}

[1] Govind P. Agrawal, Fiber-optic communication systems, second ed., John Wiley \& Sons, 1997.

[2] Robert G. Bartle, The elements of integration, John Wiley \& Sons, 1966.

[3] D. J. Blumenthal, P. Granestrand, and L. Thylen, Ber floors due to heterodyne coherent crosstalk in space photonics switches for wdm networks, IEEE Photonics Technology Letters 8 (1996), no. 2, 284-286.

[4] Michael S. Borella, Jason P. Jue, Dhritiman Banerjee, Byrav Ramamurthy, and Biswanath Mukherjee, Optical components for wdm lightwave networks, Proceedings of the IEEE 85 (1997), no. 8, 1274-1307.

[5] L. A. Buckman, L. P. Chen, and K. Y. Lau, Crosstalk penalty in all-optical distributed switching networks, IEEE Photonics Technology Letters 9 (1997), no. $2,250-252$.

[6] W. D. Cornwell and I. Androvic, Interferometric noise for a single interferer: comparison between theory and experiment, Electronics Letters 32 (1996), no. 16, $1501-1502$.

[7] W. D. Cornwell, Ivan Androvic, A. Zadok, and Moshe Tur, The role of thermal chirp in reducing interferometric noise in fiber-optic systems driven by directly 
modulated dfb lasers, IEEE Journal of Lightwave Technology 18 (2000), no. 2, 154-160.

[8] S. L. Danielsen, C. Joergensen, B. Mikkelsen, and K. E. Stubkjaer, Analysis of interferometric crosstalk in optical switch blocks using moment generating functions, IEEE Photonics Technology Letters 10 (1998), no. 11, 1635-1637.

[9] Dennis Derickson and Christian Hentschel, Fiber optic test and measurement, first ed., pp. 63-66, Prentice Hall PTR, New Jersey, 1998.

[10] Sarah Dods, Jonathan P. R. Lacey, and Rodney S. Tucker, Performance of wdm ring and bus networks in the presence of homodyne crosstalk, IEEE Journal of Lightwave Technology 17 (1999), no. 3, 388-396.

[11] L. Eskildsen, E. L. Goldstein, M. Andrejco, L. Curtis, V. Shah, D. Mahoney, C. E. Zah, and C. Lin, Interferometric noise limitations in fiber-amplifier cascades, Electronics Letters 29 (1993), no. 23, 2040-2041.

[12] G. R. Hill et al, A transport network layer based on optical network elements, IEEE Journal of Lightwave Technology 11 (1993), no. 5/6, 667-676.

[13] Daniel A. Fishman, Donald G. Duff, and Jonathan A. Nagel, Measurements and simulation of multipath interference for 1.7-gb/s lightwave transmission systems using single and multifrequency lasers, IEEE Journal of Lightwave Technology $\mathbf{8}$ (1990), no. 6, 894-905.

[14] James L. Gimlett and Nim K. Cheung, Effects of phase-to-intensity noise conversion by multiple reflections on gigabit-per-second dfb laser transmission, IEEE Journal of Lightwave Technology 7 (1989), no. 6, 888-895.

[15] E. L. Goldstein and L. Eskildsen, Scaling limitations in transparent optical networks due to low-level crosstalk, IEEE Photonics Technology Letters 7 (1995), no. 1, 93-94. 
[16] E. L. Goldstein, L. Eskildsen, and A. F. Elrefaie, Performance implications of component crosstalk in transparent lightwave networks, IEEE Photonics Technology Letters 6 (1994), no. 5, 657-660.

[17] E. L. Goldstein, L. Eskildsen C. Lin, and Y. Silberberg, Polarization statistics of crosstalk-induced noise in transparent lightwave networks, IEEE Photonics Technology Letters 7 (1995), no. 11, 1345-1347.

[18] Adilson Gonçalves, Introdução à algebra, Instituto de Matemática Pura e Aplicada (IMPA) - CNPq, 1979.

[19] Paul Richard Halmos, Naive set theory, Springer-Verlag, 1960.

[20] C. W. Helstrom, Approximate evaluation of detection probabilities in radar and optical communications, IEEE Transactions on Aerospace Electronic Systems 14 (1978), 630-640.

[21] Keang-Po Ho, Analysis of homodyne crosstalk in optical networks using gramcharlier series, IEEE Journal of Lightwave Technology 17 (1999), no. 2, 149-154.

[22] Eugenio Iannone, Roberto Sabella, Monica Avattaneo, and Gabriele de Paolis, Modeling of in-band crosstalk in wdm optical networks, IEEE Journal of Lightwave Technology 17 (1999), no. 7, 1135-1141.

[23] G. Jacobsen, K. Bertilsson, and Z. Xiaopin, Wdm transmission system performance: influence of non-gaussian detected ase noise and periodic demux characteristic, IEEE Journal of Lightwave Technology 16 (1998), no. 10, 1804-1812.

[24] Xin Jiang and Ioannis Roudas, Asimmetric probability density function of a signal with interferometric crosstalk, IEEE Photonics Technology Letters 13 (2001), no. $2,160-162$. 
[25] P. J. Legg, D. K. Hunter, I. Androvic, and P. E. Barnsley, Inter-channel crosstalk phenomena in optical time division multiplexed switching networks, IEEE Photonics Technology Letters 6 (1994), no. 5, 661-663.

[26] Peter J. Legg, Moshe Tur, and Ivan Andonovic, Solution paths to limit interferometric noise induced performance degradation in ask/direct detection lightwave networks, IEEE Journal of Lightwave Technology 14 (1996), no. 9, 1943-1954.

[27] Peter J. Legg, Moshe Tur, Hamutal Regev, W. David Cornwell, Mohammed Shabeer, and Ivan Andonovic, Interferometric noise reduction through intrabit frequency evolution of directly modulated dfb lasers, IEEE Journal of Lightwave Technology 14 (1996), no. 10, 2117-2125.

[28] Fenghai Liu, Christian J. Rasmussen, and Rune J. S. Pedersen, Analysis of homodyne crosstalk in optical networks using gram-charlier series, IEEE Photonics Technology Letters 11 (1999), no. 1, 137-139.

[29] Idelfonso Tafur Monroy and Eduward Tangdiongga, Performance evaluation of optical cross-connects by saddlepoint approximation, IEEE Journal of Lightwave Technology 16 (1998), no. 3, 317-323.

[30] Idelfonso Tafur Monroy, Eduward Tangdiongga, and Huig de Waardt, On the distribution and performance implications of filtered interferometric crosstalk in optical wdm networks, IEEE Journal of Lightwave Technology 17 (1999), no. 6, 989-997.

[31] L. Moura, N. Karafolas, A. Hill, P. Lane, and J. J. O'Reilly, Statistical modeling of interferometric crosstalk in optical networks: the race ii mundi network, Technology, Infrastructure, WDM Networks NOC 96, 1996, pp. 72-79.

[32] Arch W. Naylor and George R. Sell, Linear operator theory in engineering and science, Springer-Verlag New York Inc., 1982. 
[33] John J. O'Reilly and José R. F. Da Rocha, Improved error probability evaluation methods for direct detection optical communication systems, IEEE Transactions on Information Theory IT-33 (1987), no. 6, 839-848.

[34] Athanasios Papoulis, Probability, random variables and stochastic processes, third ed., p. 136, McGraw-Hill, New York, 1991.

[35] _ _ Probability, random variables and stochastic processes, third ed., pp. 4950, 214-218, McGraw-Hill, New York, 1991.

[36] _ Probability, random variables and stochastic processes, third ed., p. 76, McGraw-Hill, New York, 1991.

[37] Petar K. Pepeljugoski and Kam Y. Lau, Interferometric noise reduction in fiber-optic links by superposition of high frequency modulation, IEEE Journal of Lightwave Technology 10 (1992), no. 7, 957-963.

[38] Walter Rudin, Principles of mathematical analysis, McGraw-Hill, 1976.

[39] _ Real and complex analysis, McGraw-Hill, 1987.

[40] _ Functional analysis, McGraw-Hill, 1991.

[41] Yunfeng Shen, Kejie Lu, and Wanyi Gu, Coherent and incoherent crosstalk in wdm optical networks, IEEE Journal of Lightwave Technology 17 (1999), no. 5, 759-764.

[42] Claudio F. C. Silva, Rogerio Passy, Marcelo R. Jimenez, Alexandre O. Dal Forno, and Jean Pierre von der Weid, Experimental and theoretical investigations of interferometric noise power penalties in digital optical systems, IMOC'99 - Rio de Janeiro, Brasil, August 1999, pp. 344-346. 
[43] Moshe Tur and Evan L. Goldstein, Dependence of error rate on signal-to-noise ratio in fiber-optic communication systems with phase-induced intensity noise, IEEE Journal of Lightwave Technology 7 (1989), no. 12, 2055-2057.

[44] Eugene Wong and Bruce Hajek, Real and complex analysis, Springer-Verlag, 1985.

[45] Michael J. Yadlowisky and Valéria L. da Silva, Experimental comparison of the effect of discrete and distributed path inband crosstalk on system performance: application to predicting system performance penalties, IEEE Journal of Lightwave Technology 16 (1998), no. 10, 1813-1821.

[46] Amnon Yariv, Henry Blauvelt, David Huff, and Hal Zarem, An experimental and theoretical study of the suppression of interferometric noise and distortion in am optical links by phase dither, IEEE Journal of Lightwave Technology 15 (1997), no. $3,437-443$.

[47] Charles X. Yu, Weyl kuo Wang, and Stuart D. Brorson, System degradation due to multipath coherent crosstalk in wdm network nodes, IEEE Journal of Lightwave Technology 16 (1998), no. 8, 1380-1386. 


\title{
VARIÁVEIS ALEATÓRIAS CÍCLICAS E SUA APLICAÇÃO NO ESTUDO DO RUÍDO INTERFEROMÉTRICO
}

Tese de Doutorado apresentada por Marcelo Roberto Baptista Pereira Luis

Jimenez, em 17 de Setembro de 2001 ao Departamento de Engenharia Elétrica da PUC-Rio, e aprovada pela Comissão Julgadora, formada pelos seguintes membros:

\author{
Prof. Marco Antonio Grivet Mattoso Maia \\ CETUC/PUC-Rio
}

\begin{tabular}{c}
\hline Prof. Jean Pierre von der Weid \\
CETUC/PUC-Rio \\
Prof. Rogério Passy \\
CETUC/PUC-Rio \\
\hline Prof. Carlos S. Kubrusly \\
DEE/PUC-Rio \\
\hline Prof. Alexandre de Oliveira Dalforno \\
UERJ \\
\hline Erasmus Couto Brazil de Miranda \\
Intelig \\
\hline Prof. Pedro Henrique Gouvêa Coelho \\
UERJ
\end{tabular}

Visto e permitida a

impressão.

Rio de Janeiro, Pesquisa do Centro Técnico Científico 